\title{
Activation of Electron-Deficient Quinones Through Hydrogen-Bond-Donor-Coupled Electron Transfer
}

\section{Citation}

Turek, Amanda Katherine. 2015. Activation of Electron-Deficient Quinones Through HydrogenBond-Donor-Coupled Electron Transfer. Doctoral dissertation, Harvard University, Graduate School of Arts \& Sciences.

\section{Permanent link}

http://nrs.harvard.edu/urn-3:HUL.InstRepos:23845499

\section{Terms of Use}

This article was downloaded from Harvard University's DASH repository, and is made available under the terms and conditions applicable to Other Posted Material, as set forth at http:// nrs.harvard.edu/urn-3:HUL.InstRepos:dash.current.terms-of-use\#LAA

\section{Share Your Story}

The Harvard community has made this article openly available.

Please share how this access benefits you. Submit a story.

Accessibility 
(C) 2015 - Amanda Katherine Turek

All rights reserved. 


\title{
Activation of Electron-Deficient Quinones
}

\section{Through Hydrogen-Bond-Donor-Coupled Electron Transfer}

\begin{abstract}
Quinones are organic oxidants that play important roles in biological contexts and find wide application in organic synthesis. They are known to be activated toward electron transfer through hydrogen bonding, which has largely been observed for Lewis basic, weakly oxidizing quinones. Comparable activation through H-bonding is more difficult to achieve when more reactive, electron-deficient quinones are used, as these intrinsically weaker Lewis bases are less prone to engage in H-bonding interactions.

Herein, we describe the successful application of HBD-coupled electron transfer as a strategy to activate electron-deficient quinones. A systematic investigation of several smallmolecule HBDs allowed examination of the effects of $\mathrm{H}$-bonding on electron transfer to $o$ chloranil, an electron-deficient quinone that lacks the intrinsic reactivity necessary to oxidize many organic substrates of synthetic interest. This study has led to the discovery that dicationic HBDs have an exceptionally large effect on the rate and thermodynamics of these electron transfer reactions.
\end{abstract}

Favorable modulation of the thermodynamics occurs as a result of the stabilization provided to the reduced quinone $\left(\mathbf{Q}^{\bullet-}\right)$ by the HBD. Electrochemical experiments have allowed quantification of the binding affinity for $\mathbf{Q}^{\bullet-}$ to each of the HBDs, as well as elucidation of the binding stoichiometry of the resulting ground-state complex. Monocationic HBDs bind to $\mathbf{Q}^{\bullet-}$ 
with 2:1 stoichiometry, whereas dicationic HBDs bind in a 1:1 complex. Dicationic bisamidinium salts exhibit significantly improved binding to $\mathbf{Q}^{\bullet-}$, offering more thermodynamic stabilization to this reduced state.

The effects of HBDs on the kinetics of electron transfer have also been evaluated under homogenous conditions. Reactions between $o$-chloranil and ferrocene derivatives exhibit pronounced HBD-dependent rate enhancements, with dicationic HBDs displaying the greatest effect. Relative to neutral dual HBDs, the bis-amidinium salts accelerate the rate of electron transfer by $>10^{12}$. Binding stoichiometries within the rate-limiting transition states corroborate the results determined electrochemically, and binding affinity correlates with rate enhancement was observed across the series of HBDs evaluated.

Application of HBD-coupled electron transfer in an oxidative lactonization illustrates that this strategy is applicable to catalysis of organic reactions. A dicationic HBD catalyst affords the lactone product in nearly quantitative yield within $24 \mathrm{~h}$, whereas $o$-chloranil alone was ineffective $(<5 \%$ yield $)$. The rates of lactonization with several HBD catalysts correlate well with the thermodynamic and kinetic trends described above. This trend indicates that the rate of the oxidative lactonization is related to the ability of the HBD to promote an electron transfer step. Potential strategies for application in enantioselective transformations and possibilities for future mechanistic investigation are presented. 


\section{Table of Contents}

$\begin{array}{lll}\text { Abstract } & \text { iii }\end{array}$

Table of Contents $\quad$ V

$\begin{array}{ll}\text { Acknowledgments } & \text { ix }\end{array}$

List of Abbreviations and Symbols $\quad$ XV

Chapter 1. An overview of hydrogen-bonding and protonation effects in electron transfer reactions

$\begin{array}{ll}1.1 \text { Introduction } & 1\end{array}$

1.2 Proton-coupled electron 2

1.2.1 Fundamentals of PCET 2

1.2.2 PCET in organic synthesis 3

$\begin{array}{ll}1.3 \text { PCET chemistry of quinones } & 4\end{array}$

1.3.1 Mechanisms for PCET to quinones 4

1.3.2 Biological significance of PCET involving quinones 6

1.3.3 Intermediacy of H-bonded complexes in PCET mechanisms 8

1.4 Binding studies of quinones to weak HBDs 11

1.4.1 H-bonding between quinones and alcohols $\quad 11$

1.4.2 H-bonding between quinones and amides 13

1.4.3 Influence of cationic additives on electron transfer to quinones $\quad 14$

1.4.4 Binding of quinones to neutral dual HBDs 15

$\begin{array}{ll}1.5 \text { Conclusions } & 17\end{array}$ 


\section{A. Electrochemical quantification of $o$-chloranil binding to HBDs}

2a.1 Introduction

2a.2 Theoretical basis

2a.2.1 Thermodynamic analysis

2a.2.2 Mechanistic tools for the study of HBD-coupled electron transfer

2a.3 Mechanisms and notation

2a.4 Experimental design

2a.4.1 Determination of conditions for CV experiments

2a.4.2 General approach to electrochemical experiments and data analysis

2a.5 Results and discussion

2a.5.1 Mechanistic analysis of HBD-coupled electron transfer using $N, N^{\prime}$ dicyclohexylguanidinium salts

2a.5.2 Mechanistic analysis of HBD-coupled electron transfer using $N, N^{\prime}$ diphenylguanidinium salts

2a.5.3 Mechanistic analysis of HBD-coupled electron transfer using neutral ureas

2a.5.4 Comparison of binding abilities for HBDs 1-3

2a.5.5 Mechanistic analysis of HBD-coupled electron transfer using dicationic bis-amidinium salts

2a.5.6 Comparison of bis-amidinium salt $\mathbf{4}$ with HBDs $\mathbf{1 - 3}$

2a.5.7 Control experiments and comparison with bis-amidinium salt 4

2a.5.8 Effect of conformational rigidity on binding ability

2a.5.9 Mechanistic analysis of HBD-coupled electron transfer using 55 formamidinium salts 
2a.6 Conclusions from electrochemical experiments

2a.7 Experimental details

2a.7.1 General information

2a.7.2 Procedures for the syntheses of HBDs 1-9

2a.7.3 Cyclic voltammetry experiments

2a.7.4 Measurement of binding constants for neutral $\mathbf{Q}$

2a.7.5 Geometry optimizations for $(\mathrm{HBD})_{\mathrm{n}} \cdot \mathbf{Q}^{\bullet-}$ complexes

100

B. Kinetic analysis of electron transfer to o-chloranil promoted by HBDs

2b.1 Introduction

2b.2 Design of kinetic experiments

2b.3 Determination of kinetic order in HBD for electron transfer reactions

2b.4 Method of continuous variations for determination of $\mathbf{4} \cdot \mathbf{Q}$ stoichiometry

2b.5 Relative rate constants for HBD-coupled electron transfer

2b.6 Large rate acceleration provided by diphenylformamidinium salts

2b.7 Thermodynamic analysis of homogeneous electron transfer processes

2b.8 Experimental details

2b.8.1 General information

2b.8.2 Beer's Law plots

2b.8.3 Initial rates experiments for determination of order in HBD

2b.8.4 Job plot for determination of $\mathbf{4} \cdot \mathbf{Q}$ stoichiometry

2b.8.5 Pseudo-first-order experiments for determination of relative rate constants

\section{Synthetic application of HBD-coupled electron transfer}

2c.1 Introduction 
2c.2 Application of HBD-coupled electron transfer in a synthetic transformation

2c.2.1 Development and catalysis of an oxidative lactonization

2c.2.2 Structure-activity relationship studies on $\mathbf{4}$

2c.2.3 Effect of dimeric HBDs

2c.3 Kinetic isotope effect experiment and proposed mechanism

2c.4 Experimental details

2c.4.1 Procedures for the syntheses of HBDs 10-12

2c.4.2 Oxidative lactonization procedures and substrate synthesis

2c.4.3 KIE experiment

\section{Outlook for future work}

2d.1 Expanding the scope of HBD-coupled electron transfer

2d.1.1 Outlook for investigation with respect to HBD design

2d.1.2 Preliminary results for HBD-coupled electron transfer to electrondeficient $p$-quinones

2d.2 Outlook for synthetic application in enantioselective catalysis

2d.2.1 Conceptual design

2d.2.2 Oxidative lactonization catalyzed by chiral, dimeric bis-guanidinium salts

2d.3 Proposed application of HBD-coupled electron transfer in a redox-neutral reaction

2d.3.1 Reactivity principle

2d.3.2 Proposal for enantioselective approach

2d.4 Conclusions 


\section{Acknowledgments}

I have had the great fortune of having several mentors in my professional life who are not only brilliant scientists but are also wonderful human beings. First and foremost among these is my advisor, Eric Jacobsen. I've learned more from him about being a chemist, teacher, and mentor than the G1-version of me-who joined his group six years ago- ever thought I would. I am honored to have had the opportunity to study with him, and am proud of the chemist and person this opportunity has allowed me to become. Above all, I thank Eric for his unwavering confidence in me, for which I am truly grateful.

Professor Ted Betley has been a supportive member of my GAC, and in my committee meetings has challenged me to think more deeply about my science. I think one of my biggest regrets from the past six years is that I didn't get to know him better. I also thank Professor Dan Nocera for joining my thesis committee and agreeing to collaborate with us on this project. I have learned a lot from him and his group, and this project has matured greatly thanks to this collaboration. I also appreciate his enthusiasm for the project, which came at a time when it was sorely needed.

As an undergraduate, I was lucky enough to have two fantastic advisors. Professor Tehshik Yoon took me into his lab and under his wing as a sophomore. I look back on my time in the Yoon group with some embarrassment, remembering when I ran into Tehshik's office at 9 PM to show him an NMR spectrum-I had run a Swern reaction and was so excited that my crude product was clean. Despite how busy he surely was, Tehshik was nothing but enthusiastic, telling me that I was finally "a real organic chemist." He has continued to be a source of support 
and encouragement throughout my time in graduate school, always willing to lend an "ear" via email or over the phone.

Professor Bassam Shakhashiri has been a true source of inspiration to me as well. He has challenged me to think broadly about my responsibility to society as a chemist, and has taught me to understand the critical importance of clear, respectful communication with the non-science community. Bassam has traveled to Boston many times over the past six years, and I'm grateful to him for always carving out time in his schedule for me. I've enjoyed our long conversations over lunch, brunch, and dinner, and have always left feeling inspired to be a better scientist, and lucky to know Bassam as a mentor and a friend.

I thank Nicole Minotti for her encouragement and friendship over the past few years. The Jacobsen group is truly lucky to have her around, especially for help with embedding fonts. I'm especially thankful for her willingness to deal with the people from a certain company that will remain unnamed, so I didn't have to do it myself. I will miss our long-winded conversations over a wide range of topics encompassing both the mundane and (more often) the absurd.

I have been joined on my project by two collaborators. David Hardee was a welcome addition to Team Super Grover in my third year, offering support, ideas, discussion, and commiseration (when needed). Although he has since moved on to "real life," he has continued to provide advice and encouragement as I wrap up my graduate work. Andrew Ullman was my collaborator in the Nocera group, and taught me everything I know about electrochemistry. I'm appreciative of his willingness to spend countless hours teaching me about CV experiments and electrochemical simulations and discussing results, analysis, and electrochemical theory.

Rob Knowles was a terrific mentor during my first full year in the lab, always patient with me-a naïve G2 who asked too many questions. I thank him for helping me to gain 
confidence in my ideas and hypotheses, allowing me to become an independent experimentalist. He remains one of the most inspirational people I know, and I consider myself fortunate to have worked with him so closely in the Jacobsen group.

While every member of the Jacobsen group has had an impact on me in his or her own way, a few of them warrant individual acknowledgment, starting with my classmate and friend, Mike Witten. Our honest relationship has made all the difference in graduate school. Mike has been a great friend to me, always up for getting lunch at Grendel's, watching the Packers even though he's a Patriots fan, driving me and some friends to Cape Cod for my birthday, and even flying out to Milwaukee to attend my wedding.

Pam Tadross has also been a hugely supportive influence during graduate school, and has also become a very dear friend. She has patiently helped me through tough times, and always encourages me to be confident and proud of my work. Charles Yeung constantly encourages me to be optimistic, and it's hard — even for me - not to give in (at least sometimes). His advice, wisdom, and friendship have been invaluable. I'm also glad to know Baye Galligan, and I commend her for choosing a path in life that she believes will make her truly happy.

We don't get to choose our officemates and bay-mates, so I'm especially lucky to have shared a workspace with some great people. Bekka Klausen welcomed me to M202D, passing on her wisdom as a senior grad student and introducing me to Catan. I'm glad that Rose Kennedy later moved into M202D and joined me in the battle against various olfactory offenses. She is an inspiring person and I am excited to see what the future holds for her.

Gary Zhang has been my bay- and desk-neighbor for five years, which has led to many interesting discussions, both chemical and philosophical in nature. He has been consistently supportive and somehow manages to help me keep things in perspective. Although my former 
bay-mate Song Lin offered to give me foods that I'm allergic to, I still miss his face. Working across from YP has been delightful. It turns out that those shelves separating two benches don't provide much of a barrier to snarky insults. Regardless, I'll still miss his angry bump trap e-mails and lopsided fantasy football trades.

Extra thanks to the members of the Jacobsen group, both current and former, who spent some of their personal time reading over parts of this thesis and offering invaluable feedback: Rose Kennedy, David Hardee, Eric Woerly, Gary Zhang, Andrew Bendelsmith, YP (Park), and Libby Hennessy, and extra-extra thanks to Charles Yeung, who read this thesis in its entirety.

Special thanks to some old friends who made the past six years more pleasant: Tess Anderson and Souriya Vang, who were nearby in Providence and traveled to Boston for many calorie-laden visits to the dumpling house. I'm also glad that Lucy Liu went to medical school at Yale, which was very convenient for trips to New York City to see musicals, even though we didn't get Nathan Lane's autograph. Happily, I've been able to visit my former Yoon Group coworkers, Tamas Benkovics—who "adopted" me when I was an "orphan" undergraduate—and Juana $\mathrm{Du}$, in New Jersey. And I can always count on them for cheerleading via text message. I'm also thankful for friends from Wisconsin who came to visit—Julie Ripple for visiting twice and going on an adventure to Tanglewood with me, and Juli Held for forcing me to finally check out Fenway Park. I also thank Linda Cutting for helping me to sustain and develop my love of piano over the past six years.

One of the biggest challenges presented by graduate school has been the separation from my family. I can't possibly adequately thank my parents here, so I'll just say I'm certain that, with Pam and Gary Turek, I definitely won the parent lottery. I'm also grateful to my sister, Sam, for her friendship and support. Bianca and Emma are the most amazing nieces an aunt could 
hope for, and have brightened many a gloomy day. I know the distance has been especially hard on them. Grandpa and Uncle Glen are two of the most inspirational people in my life, and I strive to follow their example on how to lead a fulfilling, meaningful life. Finally, I suspect the separation may have been hardest on Grandma Judy, although our separation now is even more difficult to bear. On particularly frustrating days, a simple phone call with her would help me to get through — she always knew the right thing to say. My heart aches for her to be here and share in this accomplishment with me.

My best friend throughout graduate school—and in the eleven years I've known himhas been Wei An, who encourages me in all I do and in every instance puts my well-being above his own. Over the past six years, Wei has taken my successes and, more often, my frustrations perhaps more personally than I have. I wish our individual pursuit of professional success didn't mean that we would be separated by a thousand miles, but I'm grateful to have a partner who supports me despite this unhappy reality. Having Wei by my side-sometimes figuratively, sometimes literally—makes each day infinitely better. 
In loving memory

Judy James 


\section{List of Abbreviations and Symbols}

APCI atmospheric pressure chemical ionization

aq aqueous

$\alpha \quad$ symmetry factor

$\AA \quad$ angstrom

B3LYP Becke-3-Lee-Yang-Parr

$\mathrm{BArF}_{24}$ tetrakis[3,5-bis(trifluoromethyl)phenyl]borate

bRC bacterial reaction center

$\mathrm{BrFc} \quad$ bromoferrocene

$\mathrm{Br}_{2} \mathrm{Fc} \quad 1,1^{\prime}$-dibromoferrocene

${ }^{\circ} \mathrm{C} \quad$ degree Celsius

calcd. calculated

$\mathrm{CBz}$ benzyloxychloroformate

CE chemical step-electron transfer

CEC chemical step-electron transfer-chemical step

$\mathrm{cm} \quad$ centimeter

CV cyclic voltammetry

D diffusion coefficient

d days

d deuterium

DCM dichloromethane

DDQ 2,3-dichloro-5,6-dicyano- $p$-benzoquinone 


$\begin{array}{ll}\text { DFT } & \text { density functional theory } \\ \text { DIPEA } & \text { N,N-diisopropylethylamine } \\ \text { DME } & \text { dimethoxyethane } \\ \text { DMF } & \text { dimethylformamide } \\ \text { DMSO } & \text { dimethylsulfoxide } \\ \text { E } & \text { reduction potential } \\ \text { EC } & \text { electron transfer-chemical step } \\ \text { ECC } & \text { electron transfer-chemical step-chemical step } \\ \text { EDC } & \text { N-(3-dimethylaminopropyl)- } N \text { '-ethylcarbodiimide hydrochloride } \\ \text { equiv. } & \text { equivalents } \\ \text { ESI } & \text { electrospray ionization } \\ \text { ET } & \text { electron transfer } \\ \text { Et } 2 \text { O } & \text { diethyl ether } \\ \text { EtOAc } & \text { ethyl acetate } \\ \varepsilon & \text { molar absorptivity/extinction coefficient } \\ \text { Fc } & \text { ferrocene } \\ \text { FTIR } & \text { fourier transform infrared spectroscopy } \\ \text { G } & \text { Gibbs free energy } \\ \text { g } & \text { gram } \\ \text { h } & \text { hour } \\ \text { HBD } & \text { H-atom abstraction } \\ \text { Hz } & \end{array}$




\begin{tabular}{|c|c|}
\hline IR & infrared \\
\hline K & Kelvin \\
\hline kcal & kilocalorie \\
\hline $\mathrm{K}_{\mathrm{n}}$ & equilibrium constant \\
\hline $\mathrm{k}_{\mathrm{obs}}$ & observed rate constant \\
\hline $\mathrm{k}_{\text {rel }}$ & relative rate constant \\
\hline $\mathrm{k}_{\mathrm{s}}$ & heterogeneous rate constant \\
\hline KIE & kinetic isotope effect \\
\hline $\mathrm{L}$ & liter \\
\hline $\mathrm{m}$ & milli \\
\hline M & molar \\
\hline M06-2X & Minnesota 2006 hybrid meta density functional theory \\
\hline $\mathrm{Me}$ & methyl \\
\hline $\mathrm{Me}_{2} \mathrm{Fc}$ & 1,1'-dimethylferrocene \\
\hline $\mathrm{MeOH}$ & methanol \\
\hline MePTZ & $\mathrm{N}$-methylphenothiazine \\
\hline $\min$ & minute \\
\hline mol & mole \\
\hline MS & mass spectrometry \\
\hline$\mu$ & micro \\
\hline NBS & $N$-bromosuccinimide \\
\hline$n \mathrm{Bu}_{4} \mathrm{~N}$ & tetrabutylammonium \\
\hline $\mathrm{nm}$ & nanometer \\
\hline
\end{tabular}




$\begin{array}{ll}\text { NMR } & \text { nuclear magnetic resonance } \\ o & \text { ortho } \\ p & \text { para } \\ \text { PCET } & \text { proton-coupled electron transfer } \\ \text { PCM } & \text { polarizable continuum model } \\ \text { ppm } & \text { parts per million } \\ p \text {-Q } & p \text {-chloranil } \\ \text { PSII } & \text { photosystem II } \\ \text { Q } & o \text {-chloranil } \\ \text { rds } & \text { rate-determining step } \\ \text { rt } & \text { room temperature } \\ \text { s } & \text { second } \\ \text { sat. } & \text { saturated } \\ t \text { Bu } & \text { tert-butyl } \\ \text { TLC } & \text { thin-layer chromatography } \\ \text { THF } & \text { tetrahydrofuran } \\ \text { TBME } & \text { tert-butyl methyl ether } \\ \text { TMPD } & N, N, N \text { ', } N \text {-tetramethylphenylenediamine } \\ \text { TOF } & \text { time-of-flight } \\ \text { TolSQ } & \text { 2-tolylsulfinyl-1,4-benzoquinone } \\ \text { UV-vis } & \text { ultraviolet-visible } \\ \text { volts } \\ \text { weight percent }\end{array}$


"And this I believe: that the free, exploring mind of the individual human is the most valuable thing in the world."

John Steinbeck, East of Eden 


\section{Chapter 1}

\section{An overview of hydrogen-bonding and protonation}

\section{effects in electron transfer reactions}

\subsection{Introduction}

Hydrogen-bonding interactions have a substantial effect on the energetics of organic reactions by offering stabilizing interactions to transition structures and reactive intermediates. ${ }^{1}$ This stabilizing effect also plays an important role in electron transfer chemistry, in which $\mathrm{H}-$ bonding - and, at the extreme, protonation - can substantially alter the thermodynamics and kinetics of this reaction. ${ }^{2}$ Hydrogen-bond-donor-coupled electron transfer is a process analogous to proton-coupled electron transfer (PCET), in which H-bonding interactions - in contrast to full proton transfer-are responsible for this modulation of the thermodynamics and kinetics. In this chapter, we present a brief description of PCET and its effect on electron transfer to quinones in biological systems. An overview of the literature precedent regarding the intermediacy of $\mathrm{H}-$

\footnotetext{
${ }^{1}$ (a) Taylor, M. S.; Jacobsen, E. N. Angew. Chem. Int. Ed. 2006, 45, 1520-1543. (b) Knowles, R. R.; Jacobsen, E. N. Proc. Natl. Acad. Sci. USA 2010, 107, 20678-20685.

2 (a) Cukier, R. I.; Nocera, D. G. Annu. Rev. Phys. Chem. 1998, 49, 337-369. (b) Huynh, M. H. V.; Meyer, T. J. Chem. Rev. 2007, 107, 5004-5064. (c) Weinberg, D. R.; Gagliardi, C. J.; Hull, J. F.; Murphy, C. F.; Kent, C. A.; Westlake, B. C.; Paul, A.; Ess, D. H.; McCafferty, D. G.; Meyer, T. J. Chem. Rev. 2012, 112, 4016-4093. (d) Mayer, J, M. Annu. Rev. Phys. Chem. 2004, 55, 363-390.
} 
bonding within PCET processes is also provided, and we conclude with a review of the studies that examine the effect of synthetic hydrogen bond donors (HBDs) on electron transfer to quinones.

\subsection{Proton-coupled electron transfer}

\subsubsection{Fundamentals of PCET}

PCET has a significant impact on the thermodynamics and kinetics of many redox reactions $^{2}$ and plays a critical role in biological pathways involving radical transport and catalysis. ${ }^{3}$ The three primary mechanisms by which PCET can occur for a $1 \mathrm{H}^{+} / 1 \mathrm{e}^{-}$process are described by a square scheme (Figure 1.1). Stepwise pathways for PCET consist of two elementary steps - proton transfer and electron transfer-which can occur in either order. Irrespective of the specific route followed, the first step is generally endergonic - reduction will generate a species of increased basicity; protonation will generate a stronger oxidant. The energy differences between these two intermediates dictate the pathway by which PCET occurs, favoring the route that proceeds through the lower energy intermediate. ${ }^{4}$ In the event that both intermediates are significantly high in energy, a concerted route - in which a proton and electron are transferred concurrently-may be more favorable. However, due to the strict kinetic requirements governing such a process, concerted pathways for PCET are rarer than their stepwise counterparts. ${ }^{2 b, c}$

3 (a) Reece, S. Y.; Nocera, D. G. Annu. Rev. Biochem. 2009, 78, 673-699. (b) Kaila, V. R. I.; Verkhovsky, M. I.; Wikström, M. Chem. Rev. 2010, 110, 7062-7081. (c) Dempsey, J. L.; Winkler, J. R.; Gray, H. B. Chem. Rev. 2010, 110, 7024-7039. (d) Migliore, A.; Polizzi, N.; Therien, M. J.; Beratan, D. N. Chem. Rev, 2014, 114, 3381-3465.

${ }^{4}$ (a) Mayer, J. M.; Rhile, I. J. Biochim. Biophys. Acta 2004, 1655, 51-58. (b) Warren, J. J.; Tronic, T. A.; Mayer, J. M. Chem. Rev. 2010, 110, 6961-7001. 


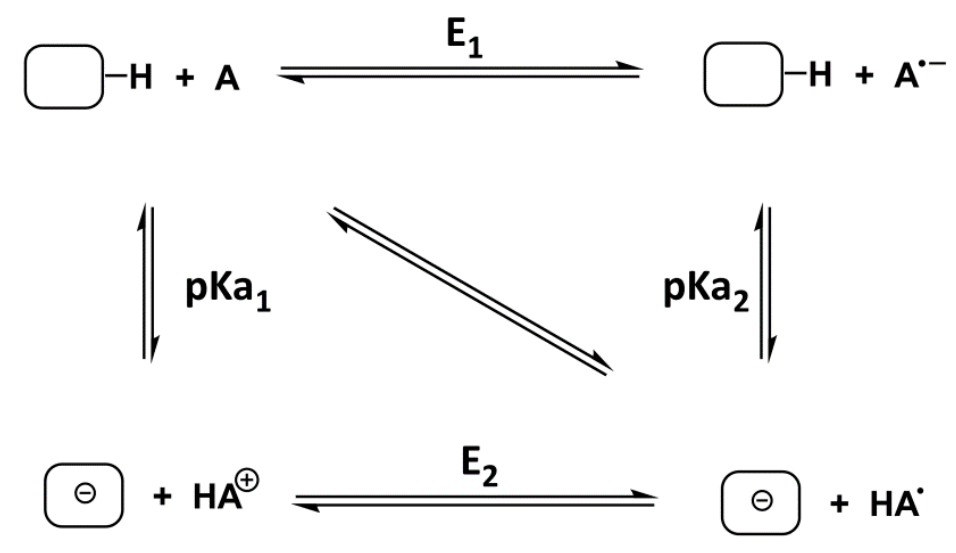

Figure 1.1. Square scheme describing pathways for PCET.

\subsubsection{PCET in organic synthesis}

PCET has recently been employed in synthetic transformations using phosphoric acid catalysts (Figure 1.2). ${ }^{5}$ In these reactions, ketones are activated toward reduction through proton transfer, with a proposed intermediate step involving H-bonding. In the absence of acid, formation of the intermediate ketyl radical is endergonic by $18 \mathrm{kcal} / \mathrm{mol}$. The incorporation of an acid catalyst, however, provides a pathway for concerted PCET, enabling reactivity that is inaccessible by direct electron transfer. Stepwise pathways are discounted on the basis of the rate law, indicating that proton transfer is involved in the rate-limiting step, and the large $\mathrm{pKa}$ differences between a protonated ketone and the acid catalyst. A chiral phosphoric acid catalyst is employed to achieve an enantioselective version of this reaction.

\footnotetext{
${ }^{5}$ Tarantino, K. T.; Liu, P.; Knowles, R. R. J. Am. Chem. Soc. 2013, 135, 10022-10025. (b) Rono, L. J.; Yayla, H. G.; Wang, D. Y.; Armstrong, M. F.; Knowles, R. R. J. Am. Chem. Soc. 2013, 135, 1773517738.
} 

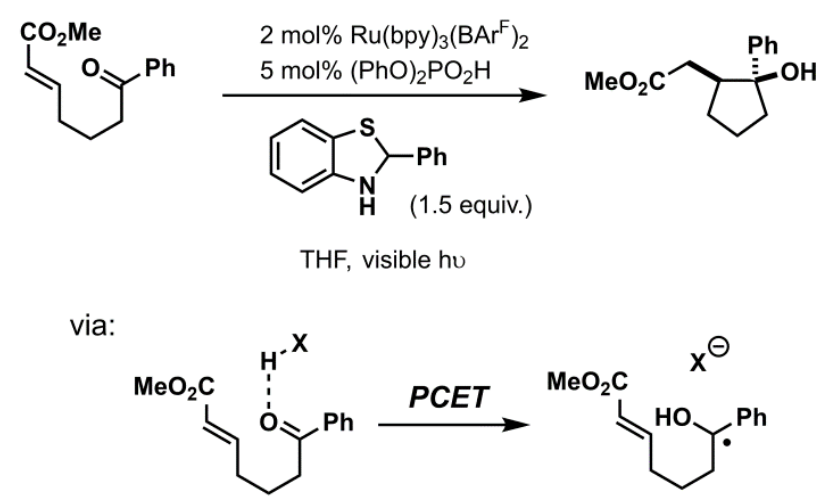

Figure 1.2. Ketyl-olefin cyclization mediated by PCET

\subsection{PCET chemistry of quinones}

\subsubsection{Mechanisms for PCET to quinones}

In aqueous solutions, quinones undergo a $2 \mathrm{H}^{+} / 2 \mathrm{e}^{-}$reduction that is described by a ninemembered square scheme (Figure 1.3). ${ }^{6}$ The specific pathway by which PCET proceeds is dependent on the $\mathrm{pH}$ of the aqueous solution, and determination of the specific mechanism can be complicated. ${ }^{7}$ Furthermore, under unbuffered aqueous conditions, the mechanistic scenarios are not limited to simple proton transfer, and H-bonding also plays a role. ${ }^{8}$

\footnotetext{
${ }^{6}$ Chambers, J. Q. Electrochemistry of Quinones. In The Chemistry of the Quinonoid Compounds; Patai, S.; Rappoport, Z.; Eds.; Wiley: New York, 1988; Vol. II, Chapter 12, pp 719-757.

${ }^{7}$ Costentin, C. Chem. Rev. 2008, 108, 2145-2179.

${ }^{8}$ Quan, M.; Sanchez, D.; Wasylkiw, M. F.; Smith, D. K. J. Am. Chem. Soc. 2007, 129, 12847-12856.
} 


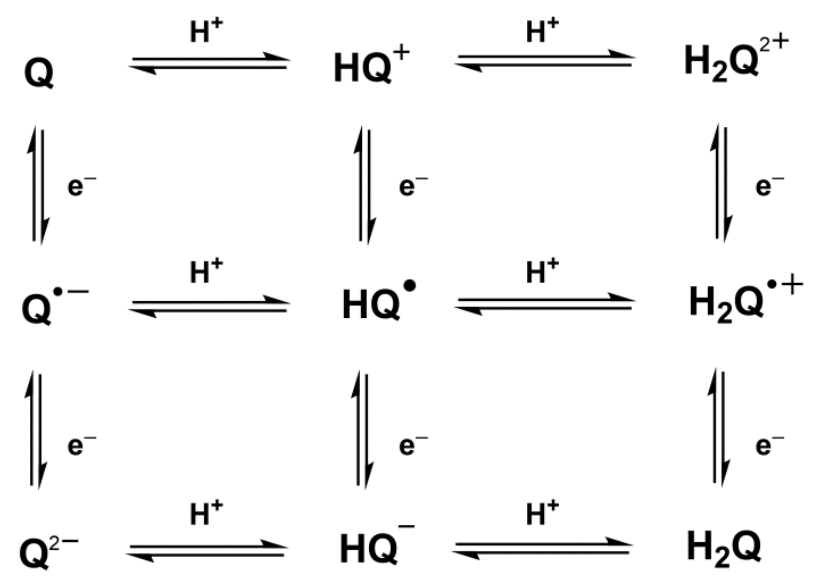

Figure 1.3. Nine-membered square scheme describing quinone reduction under aqueous or protic conditions.

Under nonaqueous, aprotic conditions, quinone reduction is considerably simplified, and proceeds through two sequential single-electron reductions, as shown on the rightmost side of the square scheme (Figure 1.4). However, in nonaqueous media, addition of protic or acidic additives can influence and complicate the mechanism of quinone reduction, ${ }^{7,9-10}$ which can again be described by the nine-membered scheme for a 2-electron reduction.

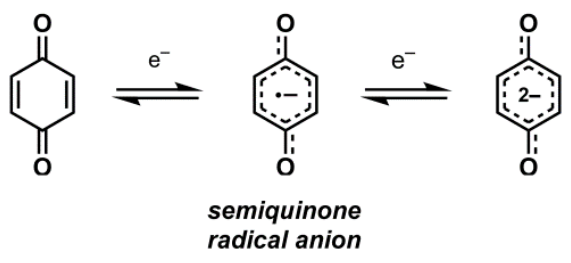

Figure 1.4. Quinone reduction in aprotic media.

\footnotetext{
${ }^{9}$ Gupta, N.; Linschitz, H. J. Am. Chem. Soc. 1997, 119, 638-6391.

${ }^{10}$ Gómez, M.; Gómez-Castro, C. Z.; Padilla-Martínez, I. I.; Martínez-Martínez, F. J.; González, F. J. J. Electroanal. Chem. 2004, 567, 269-276.
} 
Generally, concerted pathways are disfavored for reduction of quinones due to the moderate basicity of the intervening semiquinone radical anions $\left(\mathrm{Q}^{\circ-}\right) .{ }^{4}$ For this reason, quinones are often observed to proceed through a stepwise pathway involving electron transfer followed by proton transfer. In one landmark study regarding the influence of H-bonding and protonation on quinone electrochemistry, ${ }^{9}$ it was found that use of strongly H-bonding/acidic additives and/or basic quinones afforded cyclic voltammograms (CVs) that indicated H-bonding with the quinone prior to reduction. These results emphasize that the specific properties of the quinones and additives used dictate the pathways by which reduction of the quinone occurs, and will be discussed in detail in section 1.4.1.

\subsubsection{Biological significance of PCET involving quinones}

Quinones are especially important cofactors that are known to undergo PCET in many biological systems, including photosystem II (PSII) ${ }^{11}$ and the related bacterial reaction center (bRC) ${ }^{12}$ Ubiquinone (Figure 1.5) serves as the terminal electron acceptor in a chain of electron transfer events in photosynthesis, and accepts an electron from another ubiquinone molecule, $\left(\mathrm{Q}_{\mathrm{A}}\right)$. This penultimate electron acceptor, $\mathrm{Q}_{\mathrm{A}}$, behaves as an electron shuttle, consecutively transferring two electrons to $\mathrm{Q}_{\mathrm{B}}$ in a net PCET process. ${ }^{13}$

\footnotetext{
${ }^{11}$ Ferreira, K. N.; Iverson, T. M.; Maghlaoui, K.; Barber, J.; Iwata, S. Science 2004, 303, 1831-1838.

${ }^{12}$ Graige, M. S.; Paddock, M. L.; Bruce, J. M.; Feher, G.; Okamura, M. Y. J. Am. Chem. Soc. 1996, 118, 9005-9016.

${ }^{13}$ (a) Okamura, M. Y.; Paddock, M. L.; Graige, M. S.; Feher, G. Biochim. Biophys. Acta. 2000, 1458, 148-163. (b) Wraight, C. A. Front. Biosci. 2004, 9, 309-337.
} 


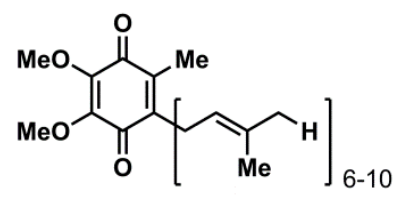

ubiquinone

Figure 1.5. General structure of ubiquinone.

Because $\mathrm{Q}_{\mathrm{A}}$ and $\mathrm{Q}_{\mathrm{B}}$ are structurally identical, there is no intrinsic difference in their reduction potentials. Despite this lack of inherent bias for electron transfer in a single direction, PCET occurs exclusively toward $\mathrm{Q}_{\mathrm{B}}$ in a stepwise manner with an initial electron transfer step. This directionality is apparently coupled to the formation of $\mathrm{H}$-bonds with $\mathrm{Q}_{\mathrm{B}}$, and more specifically, its reduced semiquinone state $\left(\mathrm{Q}_{\mathrm{B}}{ }^{-{ }^{-}}\right) .^{14}$ X-ray crystal structures obtained by Stowell for the bRC of $R b$. sphaeroides revealed that $\mathrm{Q}_{\mathrm{B}}$ undergoes a conformational shift associated with electron transfer. The structure obtained under dark conditions - under which no electron transfer occurs - showed $\mathrm{Q}_{\mathrm{B}}$ involved in a single H-bonding interaction with an amide within its binding site (Figure 1.6A). When the structure was obtained under light conditions, after electron transfer has occurred, it was found that $\mathrm{Q}_{\mathrm{B}}{ }^{\cdot-}$ reorients to accommodate additional $\mathrm{H}-$ bonding interactions within the binding site - to a cationic histidine residue and the hydroxyl side chain of a serine residue (Figure 1.6B). More recent work has ruled out the serine residue as a H-bond donor, finding instead that an additional bond forms to the amide backbone (Figure 1.6C). ${ }^{15}$ This conformational gating mechanism provides additional stabilization to $\mathrm{Q}_{\mathrm{B}}{ }^{{ }^{-}}$through

${ }^{14}$ Stowell, M. H. B.; McPhillips, T. M.; Rees, D. C.; Soltis, S. M.; Abresch, E.; Feher, G. Science 1997, $276,812-816$.

${ }^{15}$ (a) Martin, E.; Baldansuren, A.; Lin, T.-J.; Samoilova, R. I.; Wraight, C. A.; Dikanov, S. A.; O’Malley, P. J. Biochemistry 2012, 51, 9086-9093. (b) Taguchi, A. T.; O’Malley, P. J.; Wraight, C. A.; Dikanov, S. A. J. Phys. Chem. B. 2015, 119, 5805-5814. 
hydrogen-bonding, and is proposed to constitute the rate-limiting step for electron transfer to $\mathrm{QB}^{16}$

(a)

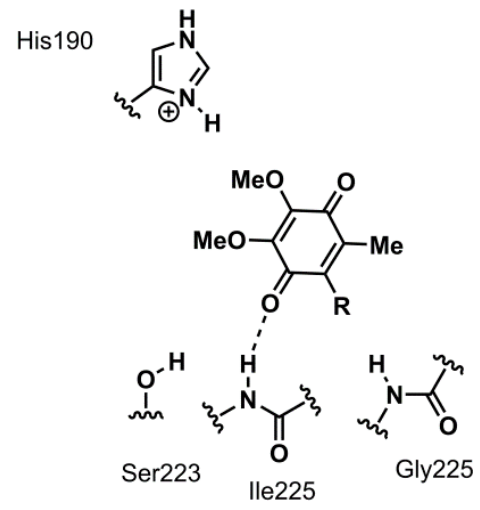

(b)

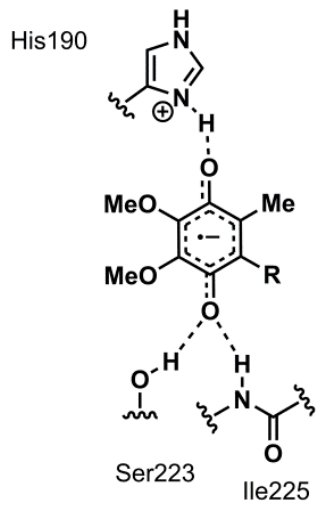

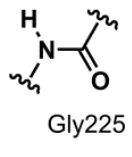

(c)

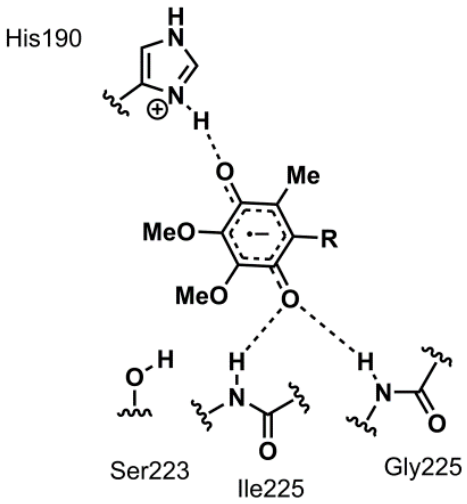

Figure 1.6. Rendition of conformational gating in the $\mathrm{Q}_{\mathrm{B}}$ binding site of $R b$. Sphaeroids. ${ }^{14}$

\subsubsection{Intermediacy of H-bonded complexes in PCET mechanisms}

Discrete H-bonded states have been found to be significant in PCET pathways, as $\mathrm{H}-$ bonding is on the continuum toward proton transfer. Electron transfer across H-bonded interfaces has played a critical role in the development of PCET theory (Figure 1.7)..$^{2 \mathrm{a}, 17}$
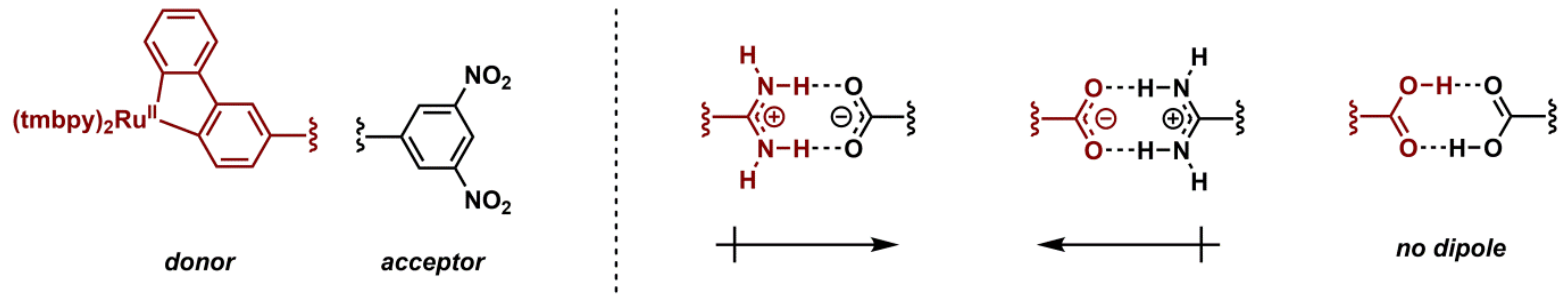

Figure 1.7. Photoinduced electron transfer across salt bridges. ${ }^{17 \mathrm{a}}$

${ }^{16}$ Graige, M. S.; Feher, G.; Okamura, M. Y. Proc. Natl. Acad. Sci. USA 1998, 95, 11679-11684.

${ }^{17}$ (a) Roberts, J. A.; Kirby, J. P.; Nocera, D. G. J. Am. Chem Soc. 1995, 117, 8051-8052. (b) Kirby, J. P.; Roberts, J. A.; Nocera, D. G. J. Am. Chem. Soc. 1997, 119, 9230-9236. (c) Young, E. R.; Rosenthal, J.; Hodgkiss, J. M.; Nocera, D. G. J. Am. Chem. Soc. 2009, 131, 7678-7684. 
Excitation of these complexes causes electron transfer to occur from the donor moiety to the acceptor moiety. Both the nature of the salt bridge and its orientation with respect to the donor and acceptor fragments influenced the rate of this electron transfer. When the electron transfer opposes the dipole of the salt bridge, PCET occurs at a faster rate and is more thermodynamically favorable than electron transfer in the direction of the dipole. Electon transfer across a symmetric salt bridge, which lacks a dipole, occurs at an intermediate rate. These results illustrate that Coulombic attraction and repulsion has a significant effect on electron transfer by affecting solvent orientation and the specific location of the protons involved. Subsequent studies demonstrated that the degree of ionization within the salt bridge, in addition to orientation of the dipole, also has a strong influence on the rate of electron transfer. The rate of electron transfer increases as the salt bridge becomes more fully ionized, though the thermodynamics appear to be unaffected. ${ }^{17 \mathrm{c}}$ The effect on rate is attributed to differences in electronic coupling between donor and acceptor, as well as to factors governing solvent reorganization.

Electrochemical studies have also been used to investigate PCET pathways proceeding through stable hydrogen-bonded intermediates. ${ }^{18}$ The urea shown in Figure 1.8 participates in a complicated PCET mechanism, with oxidation of the phenylenediamine moiety coupled to proton transfer from the urea moiety. A series of electrochemical and spectroelectrochemical experiments conclusively showed the intermediacy of a H-bonded complex in the course of this stepwise PCET mechanism, which forms after the electron transfer step and prior to proton transfer. H-bonding, however, appeared to play an important role only in less polar solvents, such as dichloromethane, and did not appear to affect the mechanism in acetonitrile. This study

${ }^{18}$ Clare, L. A.; Pham, A. T.; Magdaleno, F.; Acosta, J.; Woods, J. E.; Cooksy, A. L.; Smith, D. K. J. Am. Chem. Soc. 2013, 135, 18930-18941. 
led to the formalization of a "wedge scheme" for PCET, describing mechanisms in which these stable, long-lived H-bonded intermediates play an integral role in the overall mechanism. Mayer and coworkers have also presented a quantitative analysis of the influence of H-bonded intermediates on the overall thermodynamics and kinetics of a bimolecular PCET reaction. ${ }^{19}$

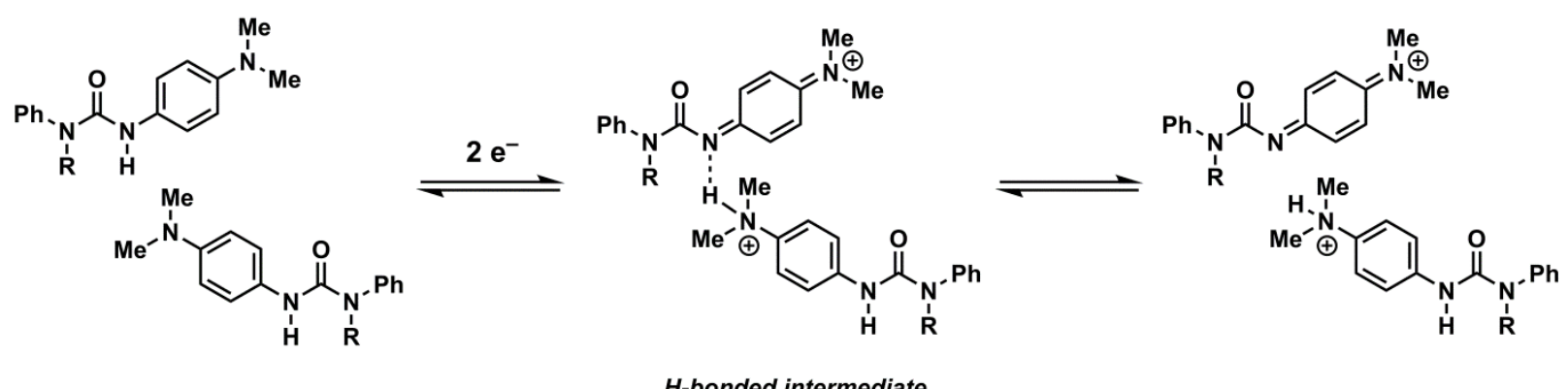

Figure 1.8. Hydrogen-bonded intermediates within a PCET mechanism. ${ }^{18}$

The involvement of H-bonded intermediates in PCET reactions of quinones has been rigorously studied in one specific example involving reduction of 3,5-di-tert-butyl-obenzoquinone. ${ }^{20}$ In the two-electron reduction of this quinone, the characteristic $\mathrm{CV}$ traces involving two reversible waves are absent, and instead are replaced with more complicated CV traces involving irreversible waves. In an attempt to elucidate the mechanism for quinone reduction in this case, formation of a stable $\mathrm{H}$-bonded intermediate between adventitious water and the semiquinone was determined to precede reduction to and protonation of the quinone dianion.

\footnotetext{
${ }^{19}$ Mader, E. A.; Mayer, J. M. Inorg. Chem. 2010, 49, 3685-3687.

${ }^{20}$ Lehmann, M. W.; Evans, D. H. J. Phys. Chem. B. 2001, 105, 8877-8884.
} 
A study of quinone reduction in unbuffered aqueous solution illustrates the importance of H-bonding in determining the mechanism by which electron transfer proceeds. ${ }^{8}$ Even in aqueous, non-acidic media, quinones undergo a one-step, two-electron reduction, largely due to the role of H-bonding. In aqueous media, the $\mathrm{CV}$ waves corresponding to the first- and second-electron reductions of quinones essentially merge into a single wave. This is attributed to a large degree of thermodynamic stabilization provided to the dianon through H-bonding with water.

\subsection{Binding studies of quinones to weak HBDs}

\subsubsection{H-bonding between quinones and alcohols}

One of the first systematic investigations of the continuum between H-bonding and proton transfer, and the corresponding effect on quinone reduction, was carried out by Linschitz and coworkers in 1997. ${ }^{9}$ This study surveyed many quinones with a broad scope of reduction potentials, and examined the effect of H-bonding additives on their electrochemistry in polar, aprotic solvents (Figure 1.9).

Weak HBDs, such as ethanol, had essentially no impact on the first electron transfer, but had a substantial impact on the second electron transfer. Determination of units describing $\mathrm{K}_{\mathrm{eq}}$ for this association is not straightforward, due to the fact that multiple alcohols can associate with the quinone and this number varies depending on the specific quinone. However, the largest effects are observed for the least oxidizing quinones, which are also the most Lewis basic. In all cases, the CVs recorded are fully reversible. 

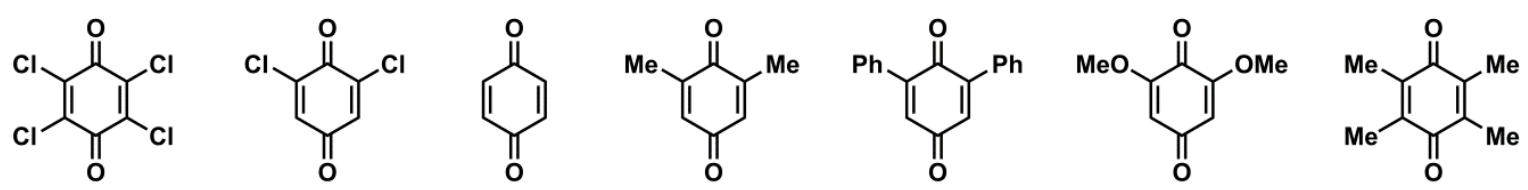

decreasing reduction potential

Figure 1.9. Quinones examined by Linschitz and coworkers. ${ }^{9}$

When stronger HBDs, such as trifluoroethanol, are used with the same quinones, a range of effects are observed by $\mathrm{CV}$, demonstrating that pathways for $\mathrm{H}$-bonding and proton transfer can manipulate the mechanisms of electron transfer and PCET. More electron-deficient quinones start to show a shift in $\mathrm{E}_{1 / 2}$ corresponding to the first electron transfer, although this effect is still quite small. The more basic quinones, however, display irreversibility in their $\mathrm{CV}$ traces, consistent with protonation of the quinone dianion post-electron-transfer. This effect is exacerbated when yet stronger H-bond donors like hexafluoroisopropanol are used, and the CV traces in this case are consistent with a mechanism involving either a protonated or hydrogenbonded complex prior to reduction to the dianion. Finally, strongly acidic donors like trifluoroacetic acid show unambiguous protonation effects consistent with a PCET pathway involving electron transfer, followed by protonation, followed by a second electron transfer. In a closely related study, this work was extended to even more basic quinones, and similar effects were observed. ${ }^{21}$

\footnotetext{
${ }^{21}$ Macías-Ruvalcaba, N. A.; González, I.; Aguilar-Martínez, M. J. Electrochem. Soc. 2004, 151, E110E118.
} 


\subsubsection{H-bonding between quinones and amides}

The effect of amides on electron transfer to $p$-chloranil, an electron-deficient quinone, has been studied as well, but still no effect on the first electron transfer was observed. ${ }^{10}$ An $o$ phenolic benzamide, capable of acting as a dual HBD, displayed the largest effect on the second electron transfer (Figure 1.10A). A separate study examined the effect of a different amide, $o$ bis(phenylcarbamoylmethyl)benzene, on electron transfer to the same quinone. ${ }^{22}$ In this case, the first electron transfer is sufficiently affected such that the quinone can mediate an electron transfer from cobalt tetraphenylporphyrin in the presence of the amide (Figure 1.10B), and the amide is determined to bind the reduced quinone in a 1:1 complex. Additionally, an effect on the rate of electron transfer was also observed, but was not quantified. However, as the reduction potentials of the two redox partners are only separated by $40 \mathrm{mV}$, the neutral quinone and electron donor are in equilibrium with the electron transfer complex. Consistent with the trends regarding Lewis basicity of quinones (vide supra), a larger effect is observed when the less electron-deficient 2,6-dichloro-p-benzoquinone is used. Under these circumstances, electron transfer is considerably less favorable $\left(\Delta \mathrm{E}_{\text {red }}=-0.21 \mathrm{~V}\right)$.

\footnotetext{
${ }^{22}$ Fukuzumi, S.; Kitaguchi, H.; Suenobu, T.; Ogo, S. Chem. Commun. 2002, 1984-1985.
} 
(a)

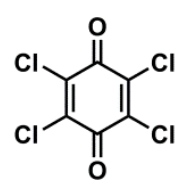

p-chloranil
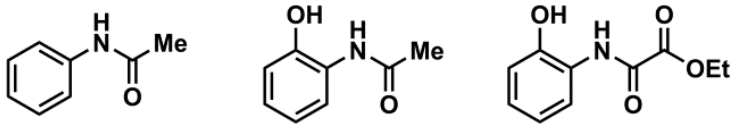

increasing effect on second reduction step

(b)
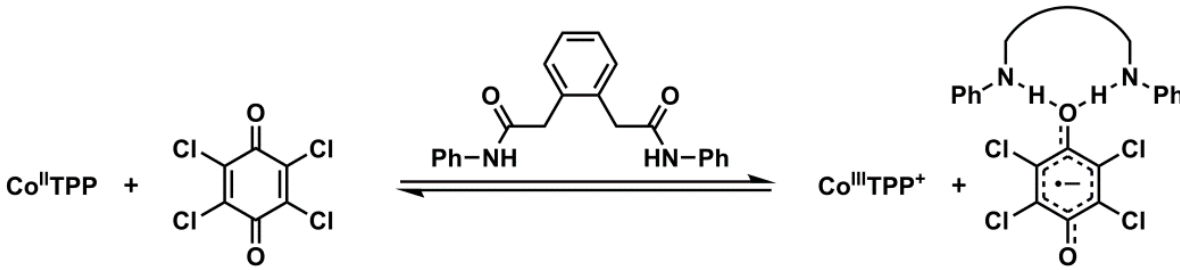

Figure 1.10. Effect of amides on electron transfer to $p$-chloranil.

\subsubsection{Influence of cationic additives on electron transfer to quinones}

Simple ammonium salts have been shown to affect the kinetics of electron transfer to benzoquinone and naphthoquinone. ${ }^{23}$ Photoinduced electron transfer from tetraphenylporphyrin to the quinone exhibits a second-order dependence on the concentration of $\mathrm{NH}_{4} \mathrm{PF}_{6}$, indicating that the reduced quinones are stabilized in a 2:1 complex with cationic ammonium ions. An effect on the reduction potentials of the quinones was also observed, although the specific binding constants in each case were not determined.

A mechanistic study designed to distinguish stepwise pathways from a concerted pathway employed protonated histidine as a means of activating a sulfinyl-substituted quinone toward reduction (Figure 1.11). ${ }^{24}$

${ }^{23}$ Okamoto, K.; Ohkubo, K.; Kadish, K.M.; Fukuzumi, S. J. Phys. Chem. A. 2004, 108, 10405-10413

${ }^{24}$ Yuasa, J.; Yamada, S.; Fukuzumi, S. J. Am. Chem. Soc. 2008, 130, 5808-5820. 

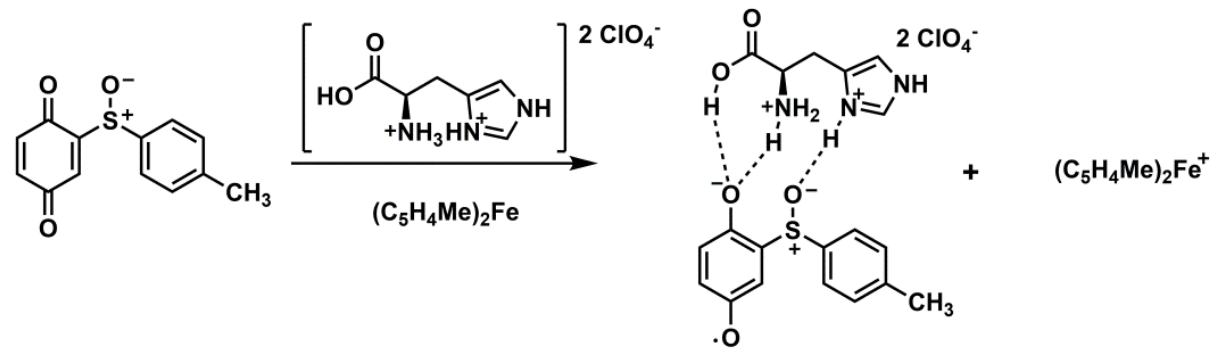

Figure 1.11. Electron transfer between TolSQ and 1,1'-dimethylferrocene, promoted by protonated histidine.

The binding constant between histidine and the quinone was calculated from a single titration point and was found to be $4.2 \times 10^{10} \mathrm{M}^{-1}$ for a 1:1 complex. This 2-tolylsulfinyl-1,4benzoquinone (TolSQ) is still a fairly weak oxidant $\left(\mathrm{E}_{1 / 2}=-0.64 \mathrm{~V} \mathrm{vs.} \mathrm{Fc}^{+/ 0}\right)$ and is quite basic, as evidenced by the irreversibility observed by cyclic voltammetry indicating that the H-bonded complex is unstable and undergoes decomposition. Protonated histidine promotes electron transfer between TolSQ and ferrocene, which in the absence of electron transfer is thermodynamically disfavored $\left(\Delta \mathrm{G}_{\mathrm{ET}}=15.5 \mathrm{kcal} / \mathrm{mol}\right)$. Other, less-reducing ferrocenes were examined in kinetic isotope effect (KIE) experiments using deuterated histidine, which revealed that the KIE decreases as driving force increases. This observation lends support for a proposed mechanistic changeover from a concerted pathway - in which H-bond formation would be part of the rate-determining step - to a stepwise pathway involving rate-determining electron transfer, in which no KIE would be observed.

\subsubsection{Binding of quinones to neutral dual HBDs}

Several studies involving binding between Lewis basic quinones and neutral dual HBDs have been performed. When 9,10-phenanthrenequinone is titrated with 1,3-diphenylurea, CV traces show that the potential corresponding to the first electron reduction experiences a positive 
shift in apparent potential (Figure 1.12A). ${ }^{25}$ In an extension of this study, it was found that this shift corresponds to a binding constant of $905 \mathrm{M}^{-1}$ describing the association between the quinone and the urea. ${ }^{26}$ It was also proposed that the structure of the quinone determines strength of binding to dual HBDs - that $o$-quinones are better suited for H-bonding as both oxygen atoms are accessible to the urea $\mathrm{N}-\mathrm{H}$ bonds. However, the potential for $p$-quinones to form $2: 1$ complexes between the HBD and the quinone appears not to have been considered. Subsequent studies with 1,4-dinitrobenzene do provide evidence for formation of these complexes. ${ }^{27}$ An interesting structure-activity relationship that demonstrates the importance of the dual H-bonding motif was carried out using pyridylureas (Figure 1.12B). 4-Pyridiylurea can provide two Hbonds to the quinone, and is as effective as 1,3-diphenylurea at stabilizing the semiquinone. However, 2-pyridylurea is expected to have an intramolecular $\mathrm{H}$-bond that renders one $\mathrm{N}-\mathrm{H}$ bond inaccessible to the quinone. As a result, this quinone has a minimal effect on the first electron reduction of the quinone.

(a)<smiles>O=C(Nc1ccccc1)Nc1ccccc1</smiles>

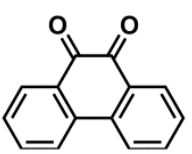

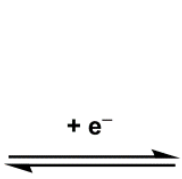

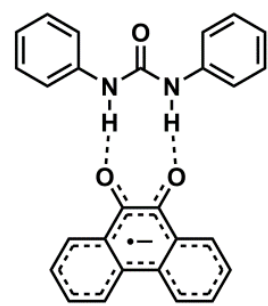

(b)

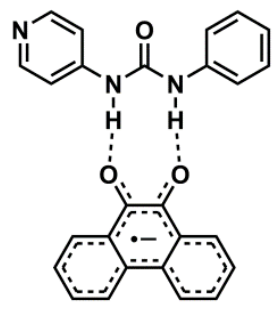

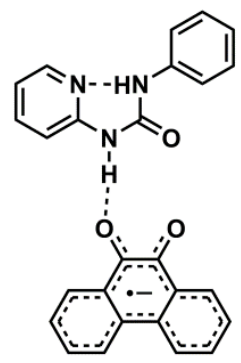

Figure 1.12. (a) Binding of 9,10-phenanthrenequinone semiquinone to 1,3-diphenylurea (b) Structure-activity relationship using 2- and 4-pyridyl-3-phenylurea.

\footnotetext{
${ }^{25}$ Ge, Y.; Lilienthal, R. R; Smith, D. K. J. Am. Chem. Soc. 1996, 118, 3976-3977.

${ }^{26}$ Ge, Y.; Miller, L.; Ouimet, T.; Smith, D. K. J. Org. Chem. 2000, 65, 8831-8838.

${ }^{27}$ Chan-Leonor, C.; Martin, S. L.; Smith, D. K. J. Org. Chem. 2005, 70, 10817-10822.
} 
Thioureas have been observed to have a measurable, albeit small, effect on the first electron transfer to ubiquinone. ${ }^{28}$ Titration of ubiquinone with one equivalent of $N, N^{\prime}$ dialkylthiourea afforded a $15 \mathrm{mV}$ shift in redox potential (the binding constant was not quantified) as a result of H-bonding between the thiourea and the semiquinone (Figure 1.13A). In contrast, diphenylthiourea — which is considerably more acidic — enabled direct two-electron reduction of ubiquinone to the dianion (Figure 1.13B). This example further demonstrates the importance of H-bonding in regulating the various mechanisms by which quinone reduction can proceed.

(a)

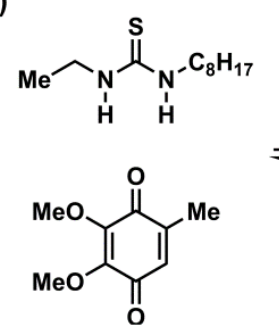

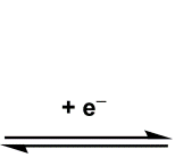

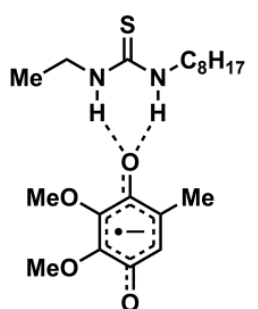

(b)

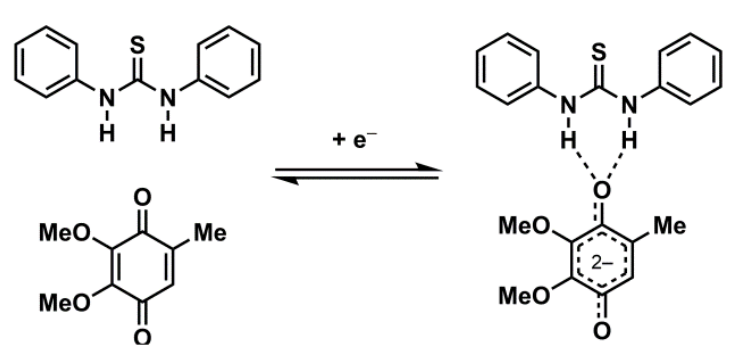

Figure 1.13. Binding of ubiquinone to thioureas.

\subsection{Conclusions}

H-bonding interactions exhibit a profound influence on electron transfer pathways, specifically when quinone oxidants are used. The studies described here have demonstrated that small-molecule HBDs are capable of stabilizing the reduced quinone through binding, thereby facilitating electron transfer. Effects on rate have also been reported. While the literature precedent describing these interactions is vast, no attempts have been made to extend this effect to a synthetically interesting context, in which quinone oxidants are used to mediate an organic

\footnotetext{
${ }^{28}$ Greaves, M. D.; Niemz, A.; Rotello, V. M. J. Am. Chem. Soc. 1999, 121, 266-267.
} 
transformation. Doing so would require both the use of synthetically useful quinones as well as design of HBDs capable of stabilizing such a quinone in its reduced state. Mechanistic studies aimed at developing such a HBD-coupled electron transfer, as well as its application in an organic reaction, are described in the subsequent chapter. 
Chapter 2

\title{
Activation of electron-deficient quinones through
}

\author{
HBD-coupled electron transfer
}

\section{A. Electrochemical quantification of $o$-chloranil binding to HBDs}

\section{2a.1 Introduction}

Because the ability of quinones to participate in electron transfer is strongly influenced by hydrogen bonding, we hypothesized that small-molecule hydrogen-bond donors (HBDs) could activate quinone oxidants for application in a synthetically interesting context. While HBDs are known to couple with electron transfer to enhance the reactivity of quinone oxidants, this effect was only observed with weakly oxidizing quinones $\left(\mathrm{E}_{1 / 2}<-0.6 \mathrm{~V}\right.$ vs. $\left.\mathrm{Fc}^{+/ 0}\right)$ that are good Lewis bases (Figure 2.1). ${ }^{1}$

\footnotetext{
${ }^{1}$ For discussion, see Chapter 1. (a) Gupta, N.; Linschitz, H. J. Am. Chem. Soc. 1997, 119, 638-6391. (b) Gómez, M.; Gómez-Castro, C. Z.; Padilla-Martínez, I. I.; Martínez-Martínez, F. J.; González, F. J. J. Electroanal. Chem. 2004, 567, 269-276. (c) Macías-Ruvalcaba, N. A.; González, I.; Aguilar-Martínez, M. J. Electrochem. Soc. 2004, 151, E110-E118. (d) Fukuzumi, S.; Kitaguchi, H.; Suenobu, T.; Ogo, S. Chem. Commun. 2002, 1984-1985. (e) Okamoto, K.; Ohkubo, K.; Kadish, K.M.; Fukuzumi, S. J. Phys. Chem. A. 2004, 108, 10405-10413. (f) Yuasa, J.; Yamada, S.; Fukuzumi, S. J. Am. Chem. Soc. 2008, 130, 58085820. (g) Ge, Y.; Lilienthal, R. R; Smith, D. K. J. Am. Chem. Soc. 1996, 118, 3976-3977. (h) Ge, Y.; Miller, L.; Ouimet, T.; Smith, D. K. J. Org. Chem. 2000, 65, 8831-8838. (i) Greaves, M. D.; Niemz, A.; Rotello, V. M. J. Am. Chem. Soc. 1999, 121, 266-267.
} 


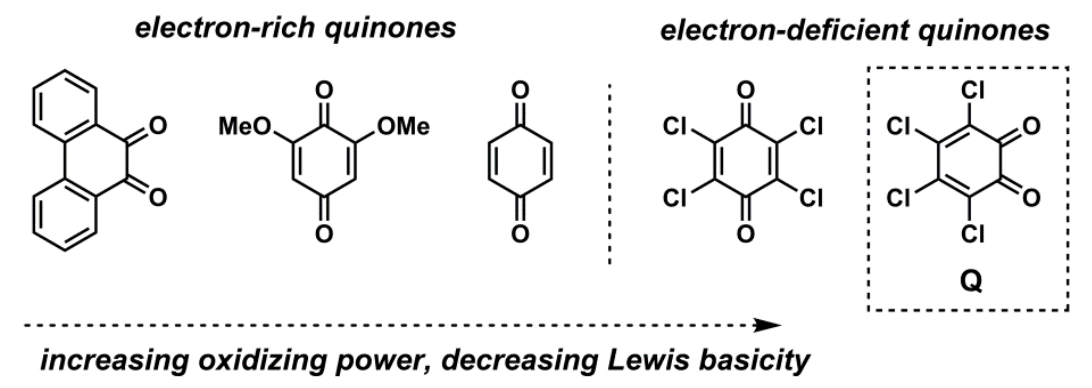

Figure 2.1. Differences between electron-rich and electron-deficient quinones.

In contrast, the HBDs studied had little discernible effect on the electron transfer to electron-deficient quinones. Electron-deficient quinones bear electron-withdrawing substituents, which increase their oxidizing ability but diminish their Lewis basicity and binding ability. In turn, this presents a challenge to the activation of electron-deficient quinones through H-bonding. This effect is even more pronounced in combination with weaker HBDs (e.g. alcohols), which are unlikely to have any discernible effect on weak hydrogen bond acceptors. However, the success of our HBD-coupled electron transfer strategy rests on the ability to activate more strongly oxidizing quinones in order to achieve synthetically useful levels of reactivity. ${ }^{2,3}$

We report herein a systematic evaluation of a series of small-molecule hydrogen bond donors, with the intention of identifying molecules that are capable of modulating the thermodynamics of electron transfer to an electron-deficient quinone (Figure 2.2). $o$-Chloranil (Q) $\left(\mathrm{E}_{1 / 2}=-0.35 \mathrm{~V}\right.$ vs. $\left.\mathrm{Fc}^{+/ 0}\right)$ was selected as the oxidant, as it is an electron-deficient quinone that nonetheless lacks the intrinsic reactivity necessary to oxidize many organic substrates of

\footnotetext{
${ }^{2}$ Becker, H.-D.; Turner, A. B. Quinones as oxidants and dehydrogenating agents. In The Chemistry of the Quinonoid Compounds; Patai, S.; Rappoport, Z.; Eds.; Wiley: New York, 1988; Vol. II, Chapter 23, pp 1352-1384.

${ }^{3}$ A detailed explanation is provided in section 2.2.
} 
synthetic interest. Our examination of the influence of H-bonding on the single-electron transfer chemistry of $o$-chloranil has led to the discovery that dicationic bis-amidinium salts can exert remarkable influence on the thermodynamics of electron transfer.

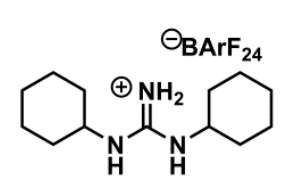

1

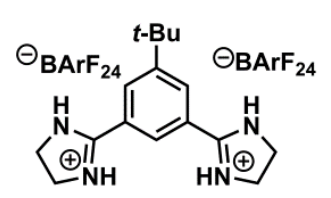

4

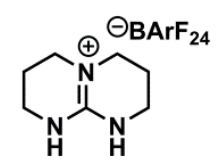

7

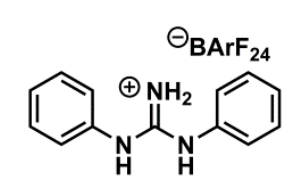

2

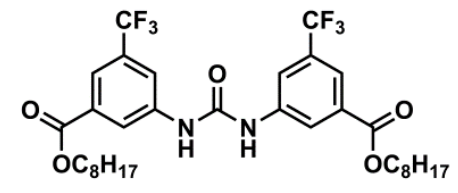

3
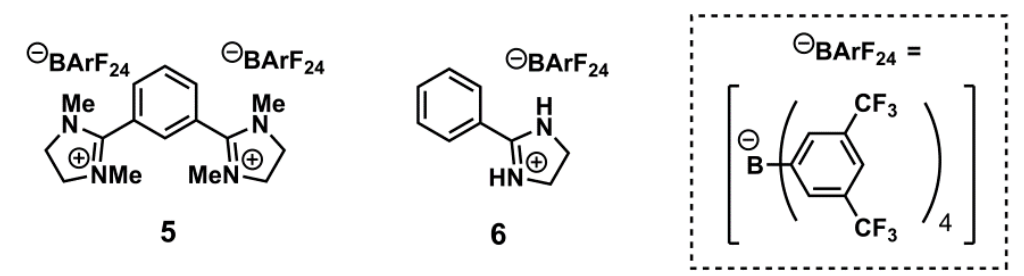

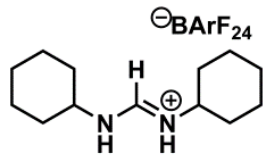

8

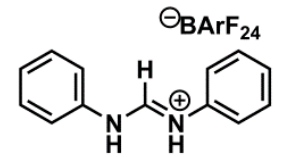

9

Figure 2.2. Series of hydrogen bond donors and additives examined in this chapter.

\section{2a.2 Theoretical basis}

\section{2a.2.1 Thermodynamic analysis}

HBD-coupled electron transfer using quinone oxidants can be split into two elementary steps: electron transfer between the quinone $(\mathbf{Q})$ and an electron donor, and binding of the reduced quinone to a HBD (Figure 2.3). The specific mechanism for this process may vary, but this general analysis aids in defining both the challenge that is presented to achieving favorable electron transfer reactions $\left(\Delta \mathrm{G}_{\text {net }}<0\right)$ using HBDs and the benefits of using electron-deficient quinones in this strategy. 

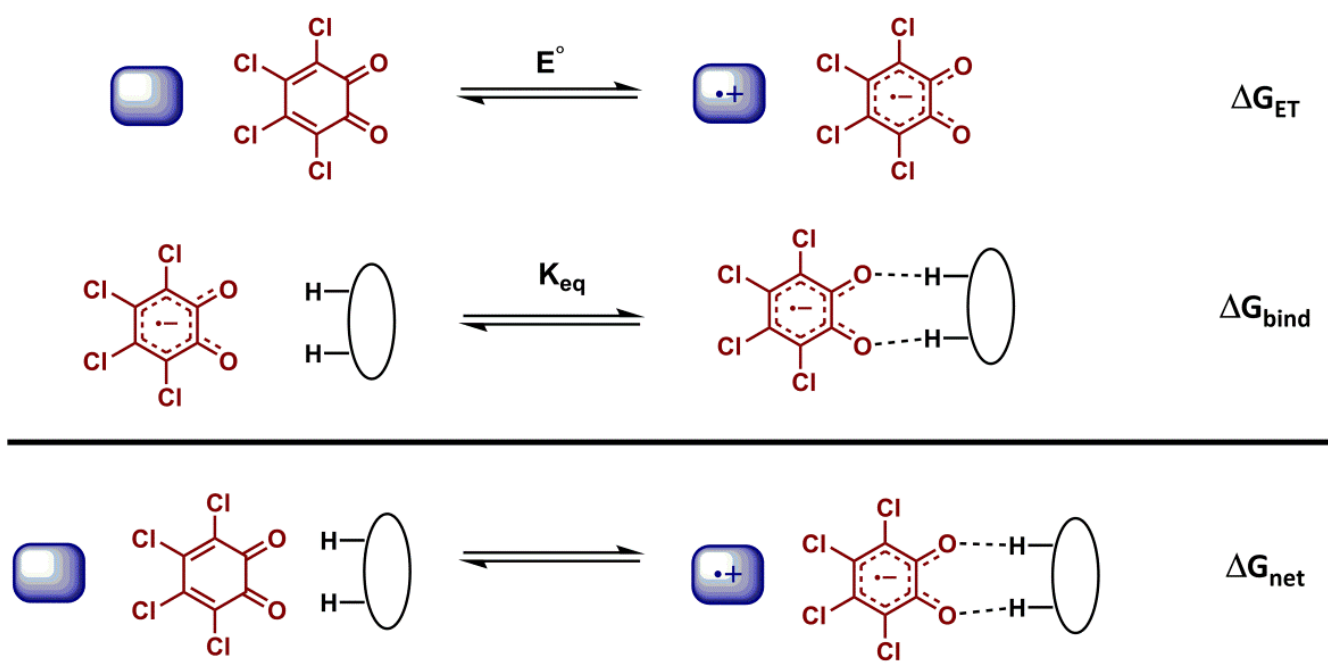

Figure 2.3. Thermodynamic analysis of hydrogen-bond-donor-coupled electron transfer.

The activating effect of a HBD on the overall reaction can be understood in terms of binding of the HBD to the reduced quinone $\left(\mathbf{Q}^{\bullet-}\right)$. This interaction $\left(\Delta \mathrm{G}_{\text {bind }}\right)$ must offset the thermodynamic price of the electron transfer (given a $\Delta \mathrm{G}_{\mathrm{ET}}>0$ ), which depends on the specific substrate and the intrinsic oxidizing ability of the quinone. Oxidation of organic substrates of interest - including olefins and aromatic rings - by electron-rich quinones is so unfavorable that an unattainably high binding energy would be necessary in order for HBDs to facilitate the overall reaction. Electron transfer in synthetically interesting contexts with electron-deficient quinones is less unfavorable. ${ }^{4}$ However, as noted above, these quinones and their reduced counterparts are inherently weak H-bond acceptors. As such, the success of the proposed HBDcoupled electron transfer strategy relies on finding the appropriate balance of HBD strength and quinone reactivity.

\footnotetext{
${ }^{4}$ Becker, H.-D.; Turner, A. B. Quinones as oxidants and dehydrogenating agents. In The Chemistry of the Quinonoid Compounds; Patai, S.; Rappoport, Z.; Eds.; Wiley: New York, 1988; Vol. II, Chapter 23, pp 1352-1384.
} 


\section{2a.2.2 Mechanistic tools for the study of HBD-coupled electron transfer}

In aprotic media, quinones undergo two sequential single electron transfers, proceeding through the semiquinone radical anion intermediate, $\mathbf{Q}^{\bullet-}{ }^{5}$ Protic and H-bonding additives influence the mechanism by which electron transfer proceeds-this study is concerned principally with the effect of HBDs on the first electron transfer step. To quantify the ability of a HBD to modulate the thermodynamics of electron transfer, we need to understand how well an HBD can bind to $\mathbf{Q}^{\bullet-}$ - or, how strongly the HBD favors this reduced state over the neutral, oxidized state.

The mechanistic tools used to quantify PCET are readily applied to the study of HBDcoupled electron transfer. Equation (1), ${ }^{6}$ which is related to the Nernst equation, shows that the apparent potential of a quinone involved in PCET will undergo a $\mathrm{pH}$-dependent shift $\left(\Delta \mathrm{E}_{1 / 2}\right)$, where $\mathrm{K}_{\mathrm{Q}}$ and $\mathrm{K}_{\mathrm{Q} \bullet-}$ correspond to the acid dissociation constants of the neutral quinone and singly-reduced semiquinone, respectively.

$$
\Delta \mathrm{E}_{1 / 2}=0.059 V \log \frac{1+\frac{\left[H^{+}\right]}{K_{Q \bullet-}}}{1+\frac{\left[H^{+}\right]}{K_{Q}}}
$$

We can adapt equation (1) to describe HBD-coupled electron transfer, in which the $\Delta \mathrm{E}_{1 / 2}$ is dependent on the HBD concentration and the association constants for the binding of the quinone and semiquinone $\left(\mathrm{K}^{\prime} \mathrm{Q}_{\mathrm{Q}}\right.$ and $\mathrm{K}^{\prime}{ }_{\mathrm{Q} \bullet-\text {, }}$, respectively) to the $\mathrm{HBD}$ (equation (2)).

\footnotetext{
${ }^{5}$ Chambers, J. Q. Electrochemistry of Quinones. In The Chemistry of the Quinonoid Compounds; Patai, S.; Rappoport, Z.; Eds.; Wiley: New York, 1988; Vol. II, Chapter 12, pp 719-757.

${ }^{6}$ (a) Costentin, C. Chem. Rev. 2008, 108, 2145-2179. (b) Costentin, C.; Robert, M.; Savéant, J.-M. Chem. Rev. 2010, 110, PR1-PR40
} 


$$
\Delta \mathrm{E}_{1 / 2}=0.059 V \log \frac{1+K^{\prime}{ }_{Q} \cdot-[H B D]}{1+K^{\prime}{ }_{Q}[H B D]}
$$

This relationship between $\Delta \mathrm{E}_{1 / 2}$ and the association constants shows that, as long as $\mathrm{K}_{\mathrm{Q}}{ }_{\bullet-}$ $>\mathrm{K}^{\prime}{ }_{\mathrm{Q}}$, increasing concentration of the $\mathrm{HBD}$ results in a more positive $\Delta \mathrm{E}_{1 / 2}$, effectively creating a more potent oxidant by favoring the reduced state through binding. The equilibrium constants that govern this shift in the potential can be elucidated electrochemically through cyclic voltammetry and provide a quantitative measure of the stabilization provided by the HBD to $\mathbf{Q}^{\bullet-}$, which in turn is related to $\Delta \mathrm{G}_{\text {bind }}$.

\section{2a.3 Mechanisms and notation}

HBDs affect the oxidizing ability of $\mathbf{Q}$ through several different pathways that can involve complex binding stoichiometries. If two HBDs are involved in binding, the square scheme is extended in order to describe all mechanistic possibilities (Figure 2.4).

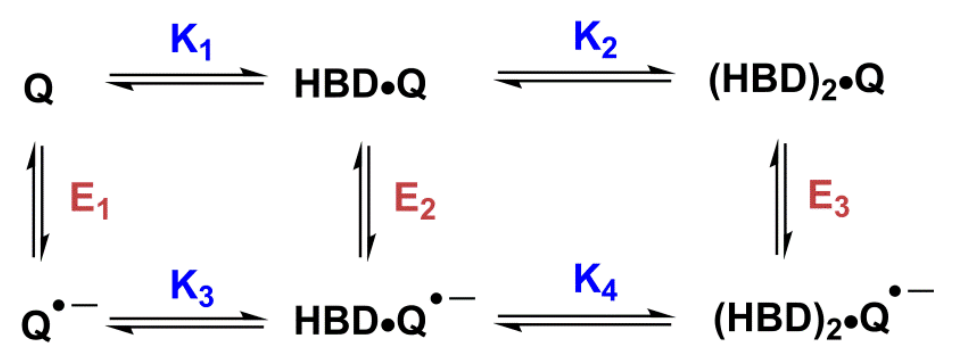

Figure 2.4. Square scheme describing HBD-coupled electron transfer to quinones when multiple binding events are involved $(\mathrm{Q}=$ quinone, $\mathrm{HBD}=$ hydrogen-bond donor $)$. 
As Figure 2.4 illustrates, a pathway for HBD-coupled electron transfer involves a series of chemical binding (C) and electron transfer (E) steps, each of which has an associated equilibrium constant. To determine the binding constants that describe the stabilization of $\mathbf{Q}^{\bullet-}$ by a given HBD, identification of the specific pathway by which HBD-coupled electron transfer proceeds and elucidation of the corresponding equilibrium constants is required. Simulations of electrochemical data can be used to distinguish between the possibilities for HBD-coupled electron transfer, and provide support for a specific mechanistic pathway. Through these simulations, values for the equilibrium constants are also obtained.

\section{2a.4 Experimental design}

\section{2a.4.1 Determination of conditions for CV experiments}

The electrochemical studies outlined in this chapter are performed with $o$-chloranil, but preliminary investigations with $p$-chloranil $(\boldsymbol{p}$-Q) provided the basis for determination of our experimental conditions. Selection of an appropriate electrolyte was critical as the identity of the electrolyte proved to have a significant impact on the CV data. For example, when $n \mathrm{Bu}_{4} \mathrm{NPF}_{6}$ was employed as the electrolyte, a minimal effect on the apparent potential of the $p-\mathbf{Q} / \mathbf{p}-\mathbf{Q}^{\bullet-}$ couple was observed over the course of a titration with 1 (Figure 2.5). 


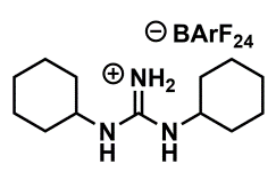

1

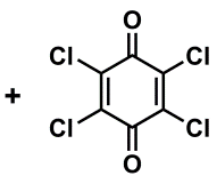

p-chloranil

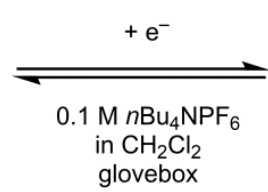

glovebox<smiles>NC(=O)NC1CC2CC3CC(C2)CC1C3</smiles>

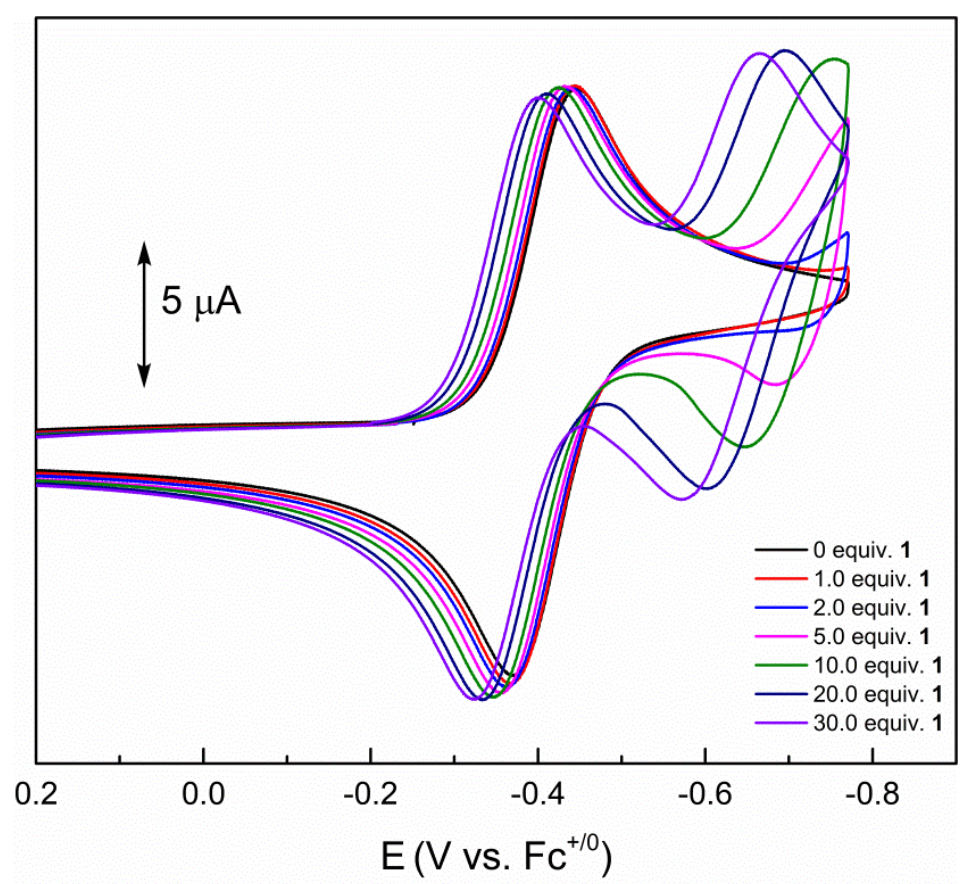

Figure 2.5. Experimental CVs of $p$-chloranil (initial concentration $=0.5 \mathrm{mM}$ ) recorded in $0.1 \mathrm{M}$ $n \mathrm{Bu}_{4} \mathrm{PF}_{6} / \mathrm{CH}_{2} \mathrm{Cl}_{2}$ (glovebox) in the presence of increasing [1].

Speculating that this small effect was due to inhibition of the $\mathrm{HBD}$ by ${ }^{-} \mathrm{PF}_{6}$, we repeated this experiment using an electrolyte with a non-coordinating anion. ${ }^{7}$ A switch to $n \mathrm{Bu}_{4} \mathrm{NBArF}_{24}{ }^{8}$ affords more substantial shifts in the recorded CVs of $\boldsymbol{p}$-Q upon titration with 1 (Figure 2.6).

${ }^{7}$ (a) LeSuer, R. J.; Buttolph, C.; Geiger, W. E. Anal. Chem. 2004, 76, 6395-6401. (b) Barrière, F.; Geiger, W. E. J. Am. Chem. Soc. 2006, 128, 3980-3989.

${ }^{8}$ Nishida, H.; Takada, N.; Yoshimura, M.; Sonoda, T.; Kobayashi, H. Bull. Chem. Soc. Jpn. 1984, 57, 2600-2604. 


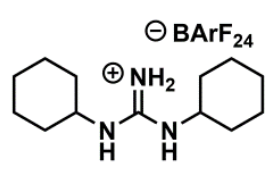

1

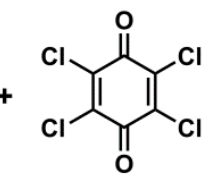

p-chloranil

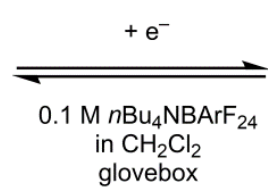

glovebox<smiles>CC(C)(C)C1C2CC3CC(C2)CC1C3</smiles><smiles></smiles>

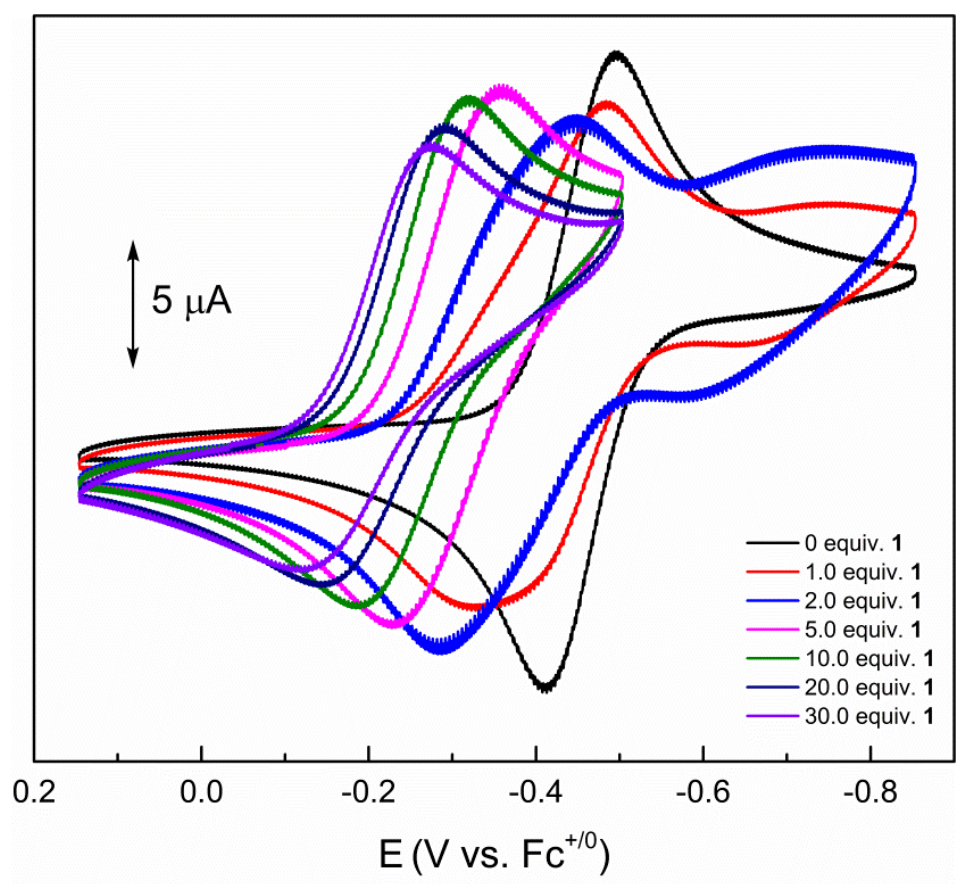

Figure 2.6. Experimental CVs of $p$-chloranil (initial concentration $=0.5 \mathrm{mM}$ ) recorded in $0.1 \mathrm{M}$ $n \mathrm{Bu}_{4} \mathrm{NBArF}_{24} / \mathrm{CH}_{2} \mathrm{Cl}_{2}$ (glovebox) in the presence of increasing [1].

Choice of solvent also proved critical in these cyclic voltammetry studies. Polar solvents such as acetonitrile are potent inhibitors of HBDs, and previous experiments using UV-vis showed no effect of $\mathrm{H}$-bonding on electron transfer to quinones in acetonitrile and acetone. Small effects were also observed with tetrahydrofuran, which can likely also be attributed to inhibition of the HBD by a Lewis basic solvent. 


\section{2a.4.2 General approach to electrochemical experiments and data analysis}

A systematic investigation of HBDs was undertaken, with the aim of understanding how H-bonding interactions can stabilize $\mathbf{Q}^{--}$and thereby promote electron transfer to electrondeficient quinones are used. Titrations of $\mathbf{Q}$ were carried out with each HBD, using cyclic voltammetry to record the $\Delta \mathrm{E}_{1 / 2}$. In each case, a significant, measurable effect was observed on the apparent potential that corresponds to the first electron transfer. Additionally, the reversibility of the CVs recorded in all titration experiments indicate that the $\Delta \mathrm{E}_{1 / 2}$ is the result of $\mathrm{H}$-bonding to $\mathbf{Q}^{--}$and not protonation, which would manifest as irreversibility in the CV traces.

The experimental data in each case were analyzed using simulations to determine the pathway and equilibrium constants associated with HBD-coupled electron transfer. The equations and parameters used for each simulation are outlined in the subsequent sections. Discrepancies in current magnitude between experiment and simulation are noted throughout the various titration experiments, and are attributed to variability in diffusion coefficient across the range of species involved in the simulation. This value, which was not determined by the simulations, has no bearing on $\Delta \mathrm{E}_{1 / 2}$ and therefore does not impact the simulated equilibrium constants.

\section{2a.5 Results and discussion}

\section{2a.5.1 Mechanistic analysis of HBD-coupled electron transfer using N,N'- dicyclohexylguanidinium salts}

Titration of $\mathbf{Q}$ with $N, N^{\prime}$-dicyclohexylguanidinium salt $\mathbf{1}$ afforded the cyclic voltammetry traces shown in Figure 2.7. 


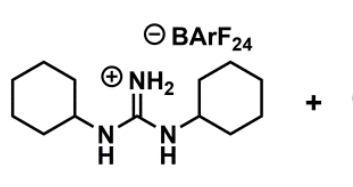

1

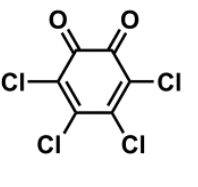

$\mathbf{Q}$

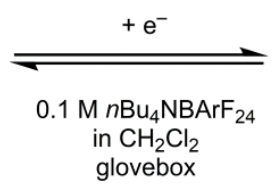

glovebox
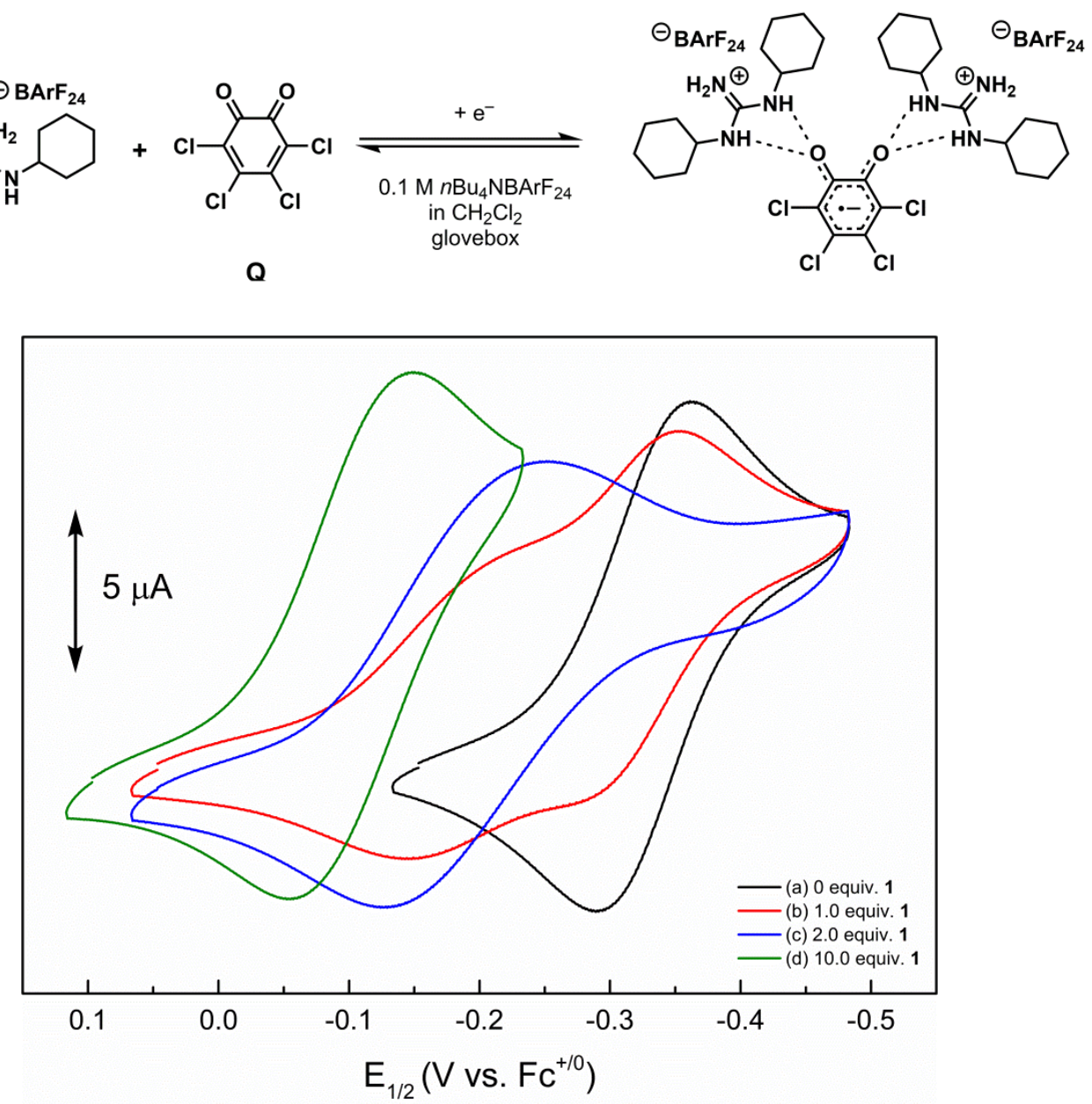

Figure 2.7. Experimental $\mathrm{CVs}$ of $o$-chloranil (initial concentration $=0.5 \mathrm{mM}$ ) recorded in $0.1 \mathrm{M}$ $n \mathrm{Bu}_{4} \mathrm{NBArF}_{24} / \mathrm{CH}_{2} \mathrm{Cl}_{2}$ (glovebox) in the presence of increasing [1].

To elucidate the equilibrium constants that describe binding of $\mathbf{Q}^{\bullet-}$ to $\mathbf{1}$, the full set of electrochemical data for this titration were subjected to simulations. The CVs recorded at low [1] present distinct curve shapes that aided in determining a starting point for the simulations (Figure 2.8). As [1] increases, a current peak at higher potential $(-0.25 \mathrm{~V})$ increases at the expense of the current peak at lower potential $(-0.35 \mathrm{~V})$. This indicates that the mechanism for HBD-coupled electron transfer between $\mathbf{Q}$ and $\mathbf{1}$ may involve pre-association (prior to electron transfer) between the two species. If this pre-association is significant, addition of $\mathbf{1}$ will cause $\mathbf{Q}$ to partition into the bound state $\mathbf{1} \cdot \mathbf{Q}$ and unbound state $\mathbf{Q}$. The increase in $[\mathbf{1} \cdot \mathbf{Q}]$ at the expense of 
[Q] would afford a corresponding increase in current due to reduction of this complex, with a concomitant decrease in current due to reduction of $\mathbf{Q}$.

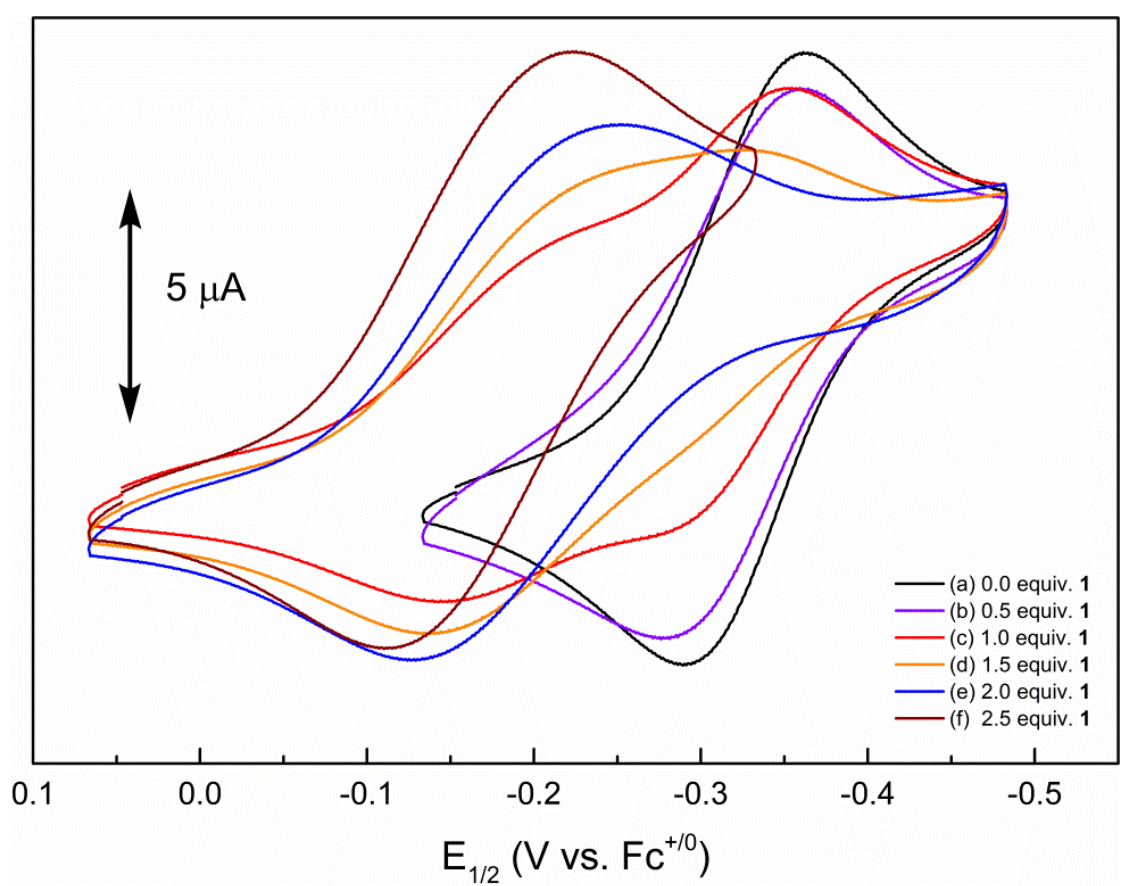

Figure 2.8. Experimental CVs of $o$-chloranil (initial concentration $=0.5 \mathrm{mM}$ ) recorded in $0.1 \mathrm{M}$ $n \mathrm{Bu}_{4} \mathrm{NBArF}_{24} / \mathrm{CH}_{2} \mathrm{Cl}_{2}$ (glovebox) in the presence of increasing [1]. Only data collected at low [1] are shown.

This pre-association hypothesis was borne out in the simulations, in which a mechanism involving binding of neutral $\mathbf{Q}$ to $\mathbf{1}$ provides the best fit to the experimental data. Specifically, these data are accurately represented by a CEC mechanism, in which this binding event $\left(\mathrm{K}_{1}\right)$ precedes electron transfer $\left(\mathrm{E}_{2}\right)$, and another binding event between $\mathbf{1}$ and $\mathbf{Q}^{\circ-}$ follows $\left(\mathrm{K}_{4}\right)$ (Figure 2.9). These simulations reproduce the experimental data with respect to the overall $\Delta \mathrm{E}_{1 / 2}$ (Figure 2.10), and also reproduce the distinct features of the CVs at low [1] as in Figure 2.11. Investigations into the binding equilibrium between $\mathbf{1}$ and $\mathbf{Q}$ using spectroscopic methods qualitatively indicate an association between the two species. Independently determined $\mathrm{K}_{1}$ 
values using a Benesi-Hildebrand analysis are consistent with those obtained from the simulations, providing additional support for the CEC mechanism. These data, along with simulated mechanisms that represent poor fits to the experimental data, are presented in section 2.7.3.

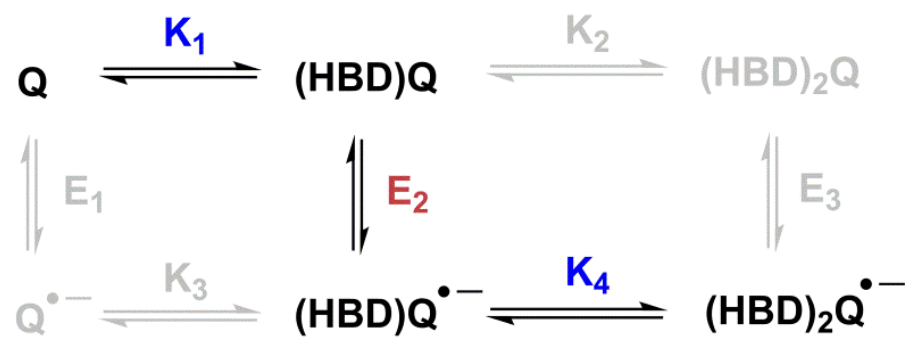

Figure 2.9. CEC mechanism.

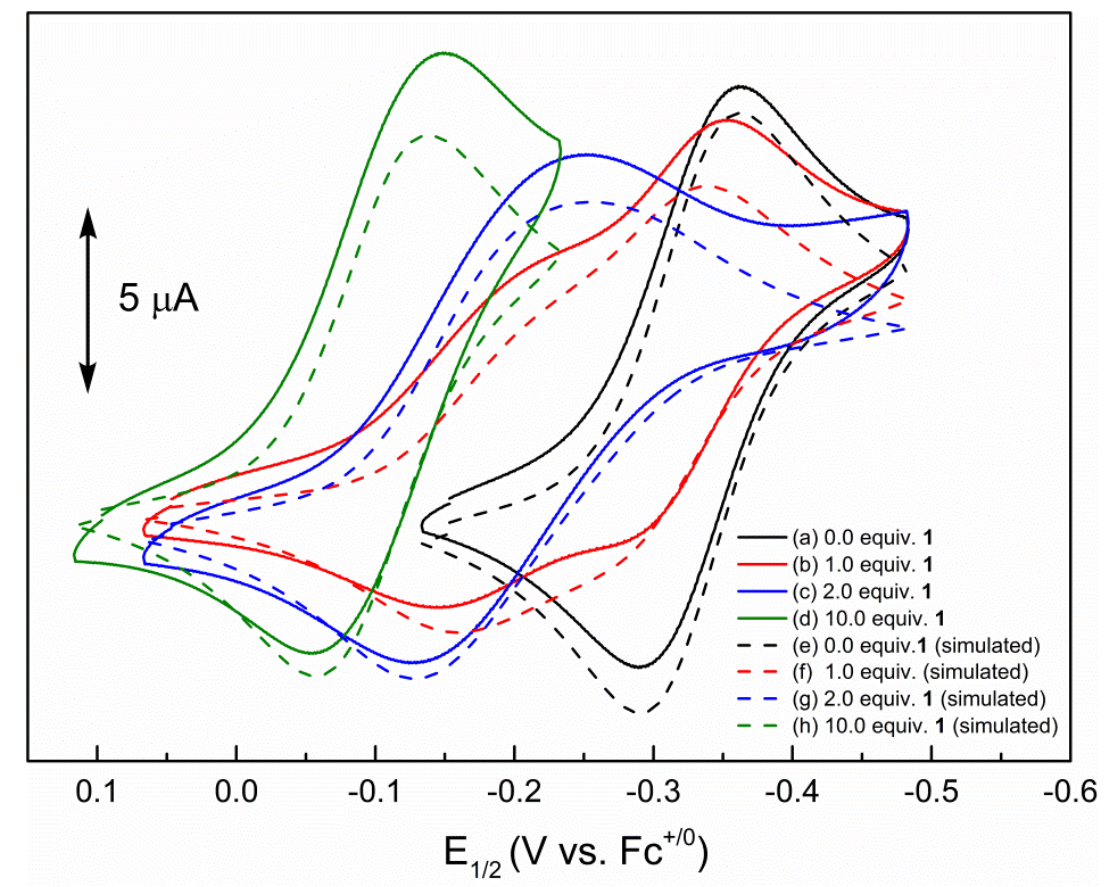

Figure 2.10. Experimental $\mathrm{CVs}$ of $o$-chloranil (initial concentration $=0.5 \mathrm{mM}$ ) recorded in 0.1 $\mathrm{M} n \mathrm{Bu}_{4} \mathrm{NBArF}_{24} / \mathrm{CH}_{2} \mathrm{Cl}_{2}$ (glovebox) in the presence of increasing [1] and comparison with simulation of a CEC mechanism. 


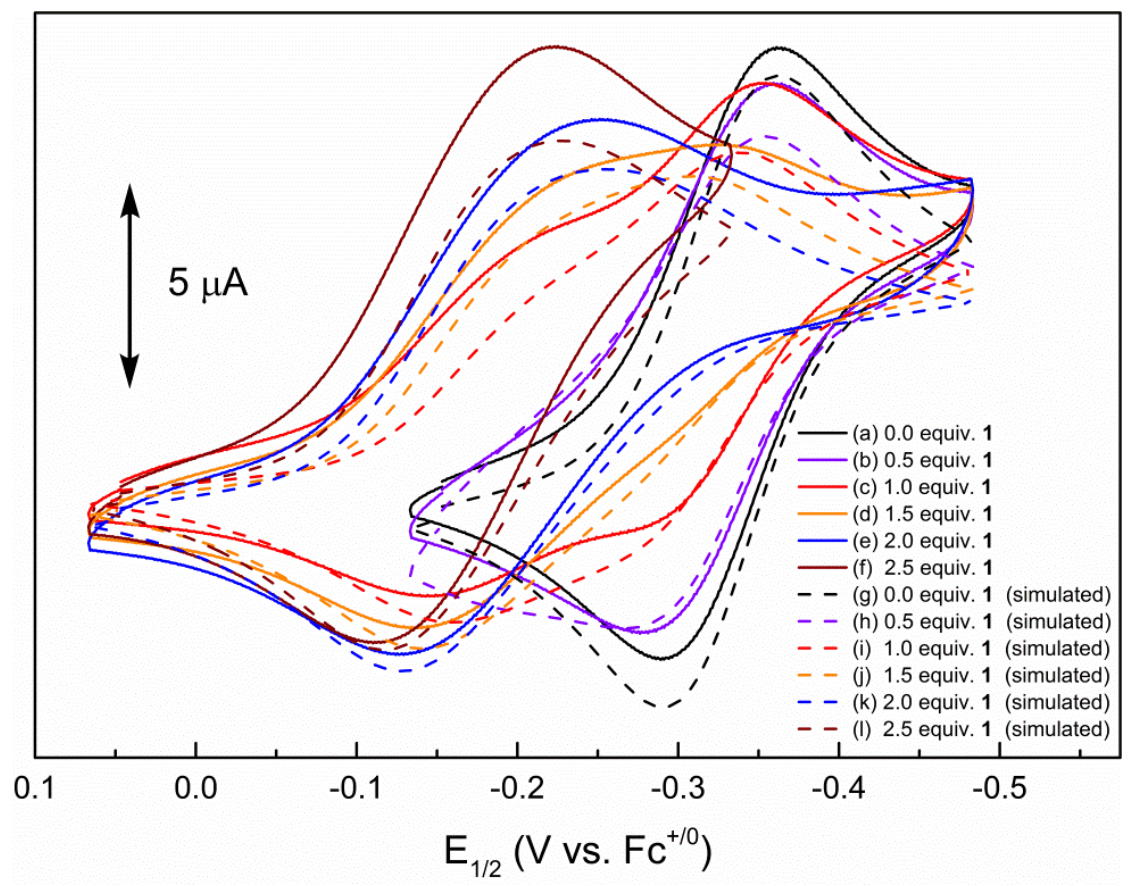

Figure 2.11. Experimental CVs of $o$-chloranil (initial concentration $=0.5 \mathrm{mM}$ ) recorded in 0.1 $\mathrm{M} n \mathrm{Bu}_{4} \mathrm{NBArF}_{24} / \mathrm{CH}_{2} \mathrm{Cl}_{2}$ (glovebox) in the presence of increasing [1] and comparison with simulation of a CEC mechanism. Only data collected at low [1] are shown.

Simple DFT calculations indicate that the geometry of the $(\mathbf{1})_{2} \cdot \mathbf{Q}^{\bullet-}$ complex involves bifurcated H-bonding interactions, in which each quinone oxygen is bound to a different HBD molecule (Figure 2.12), with H-bonding distances of $1.81 \AA$ and $1.67 \AA$ calculated with B3LYP, and $1.77 \AA$ and $1.98 \AA$ with M06-2X. Other input geometries-for example, in which each HBD binds to an oxygen and chlorine in a four-centered interaction-converge to these structures. Computations performed using two different functionals orient the HBDs differently relative to each other, although both show this bifurcated H-bond interaction with $\mathbf{Q}^{\bullet-}$. 
(a)
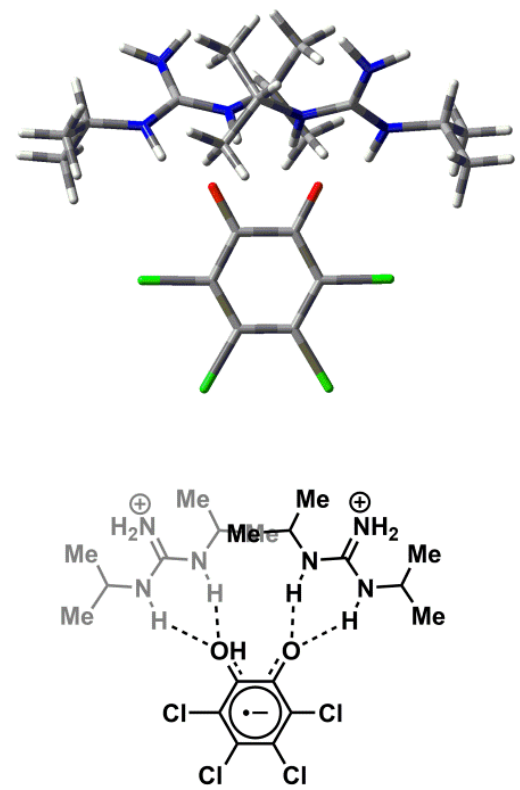

(b)
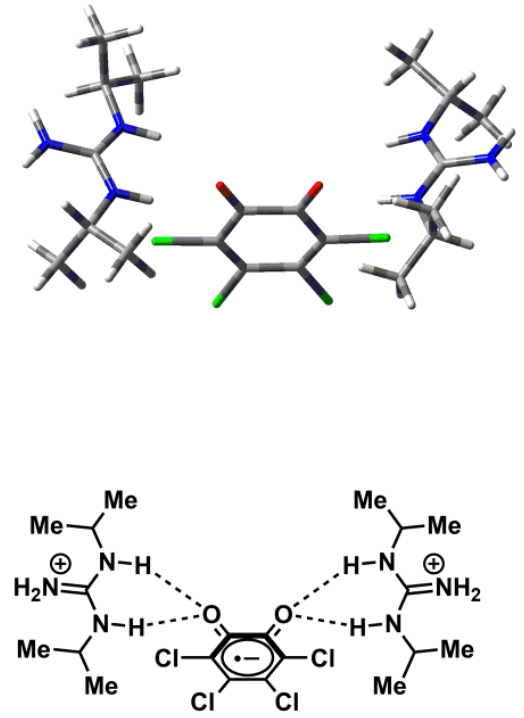

Figure 2.12. Geometry calculated for the $(\mathbf{1})_{2} \cdot \mathbf{Q}^{\bullet-}$ complex, optimized using (a) B3LYP/6$31 \mathrm{G}(\mathrm{d}, \mathrm{p})$ level of theory; the gray HBD molecule is oriented behind the other HBD molecule; (b) M06-2X/6-31G(d,p) level of theory.

The bottom of the extended square scheme in Figure 2.4 describes the stabilization of $\mathbf{Q}^{\bullet-}$ through binding to two HBD molecules. Therefore, $\mathrm{K}_{3} \mathrm{~K}_{4}$ - the product of the equilibrium constants corresponding to the two binding events - quantitatively describes the equilibrium favoring the reduced state and provides a measure of the oxidizing strength of $\mathbf{Q}$ in the presence of a given HBD. Explicit determination of $\mathrm{K}_{1}, \mathrm{E}_{2}$, and $\mathrm{K}_{4}$ is afforded by simulation of a CEC mechanism, and $\mathrm{E}_{1}$ is experimentally determined. Therefore, the thermodynamically redundant parameter $\mathrm{K}_{3}$ can be calculated from these values. The equations and parameters used to simulate the experimental data, including the elucidated equilibrium constants, are shown in Table 2.1. ${ }^{9}$ From these values, $\mathrm{K}_{3} \mathrm{~K}_{4}$ describing formation of the $(\mathbf{1})_{2} \cdot \mathbf{Q}^{\bullet-}$ complex was calculated to be $6.1 \times 10^{8} \mathrm{M}^{-2}$.

\footnotetext{
${ }^{9}$ In this simulation and subsequent simulations, $\mathrm{k}_{\mathrm{f}}$ for each binding step was fixed at the diffusioncontrolled limit of $1 \times 10^{10} \mathrm{~s}^{-1}$. This was due to the fact that the $\mathrm{k}_{\mathrm{f}}$ was consistently simulated to be slightly faster than the diffusion-controlled limit, so it was fixed at the fastest reasonable rate.
} 
Table 2.1. Equations and best-fit parameters used to simulate $\mathrm{CV}$ data for $0.5 \mathrm{mM} o$-chloranil $(\mathbf{Q})$ in $0.1 \mathrm{M} n \mathrm{Bu}_{4} \mathrm{NBArF}_{24} / \mathrm{CH}_{2} \mathrm{Cl}_{2}$ (glovebox) in the presence of increasing [1]. Italicized values were fixed in the simulation.

\begin{tabular}{cccc}
\hline $\begin{array}{c}\text { Electron transfer } \\
\text { steps }\end{array}$ & $\mathbf{E}_{\mathbf{1} / \mathbf{2}}\left(\mathbf{V} \mathbf{v s .} \mathbf{F} \mathbf{F}^{+/ 0}\right)$ & $\boldsymbol{\alpha}$ & $\mathbf{k}_{\mathbf{s}}(\mathbf{c m} / \mathbf{s})$ \\
\hline $\mathbf{Q}+\mathrm{e}^{-} \rightarrow \mathbf{Q}^{\bullet-}$ & -0.33 & 0.5 & 0.022 \\
$\mathbf{1} \cdot \mathbf{Q}+\mathrm{e}^{-} \rightarrow \mathbf{1} \cdot \mathbf{Q}^{\cdot-}$ & -0.17 & 0.5 & 0.21 \\
\hline $\mathbf{C h e m i c a l ~ s t e p s}$ & $\mathbf{K}_{\mathbf{e q}}\left(\mathbf{M}^{-1}\right)$ & & $\mathbf{k}_{\mathbf{f}}\left(\mathbf{s}^{-1}\right)$ \\
\hline $\mathbf{Q} \rightarrow \mathbf{1} \cdot \mathbf{Q}$ & 66 & $1 \times 10^{10}$ \\
$\mathbf{1}+\mathbf{1} \cdot \mathbf{Q}^{--} \rightarrow(\mathbf{1})_{\mathbf{2}} \cdot \mathbf{Q}^{--}$ & $1.8 \times 10^{4}$ & & $1 \times 10^{10}$
\end{tabular}

2a.5.2. Mechanistic analysis of HBD-coupled electron transfer using $N, N$ '-diphenylguanidinium salts

Titration of $\mathbf{Q}$ with $N, N^{\prime}$-diphenylguanidinium salt $\mathbf{2}$ also affords a shift in the apparent potential of the $\mathbf{Q} / \mathbf{Q}^{\bullet-}$ redox couple (Figure 2.13). At low [2], the recorded CVs display features similar to those observed in the titration with 1 (Figure 2.14), indicating that HBD-coupled electron transfer involving $\mathbf{2}$ may also proceed via a CEC mechanism. In addition to these curve shapes, other current peaks are observed at low potential as [2] increases (e.g. scans (c)-(d) in Figure 2.13). These peaks correspond to the second electron reduction of $\mathbf{Q}$ (e.g. the $\mathbf{Q}^{\bullet-} / \mathbf{Q}^{2-}$ redox couple). The apparent potential corresponding to this process undergoes a substantial shift upon addition of $\mathbf{2}$, due to the strong Lewis basicity of the dianion. Because we are only concerned with the effect of HBDs on the first electron transfer, we did not attempt to simulate this second CV wave. 
<smiles>[NH3+]C(Nc1ccccc1)Nc1ccc(C(F)(F)F)cc1</smiles>

2
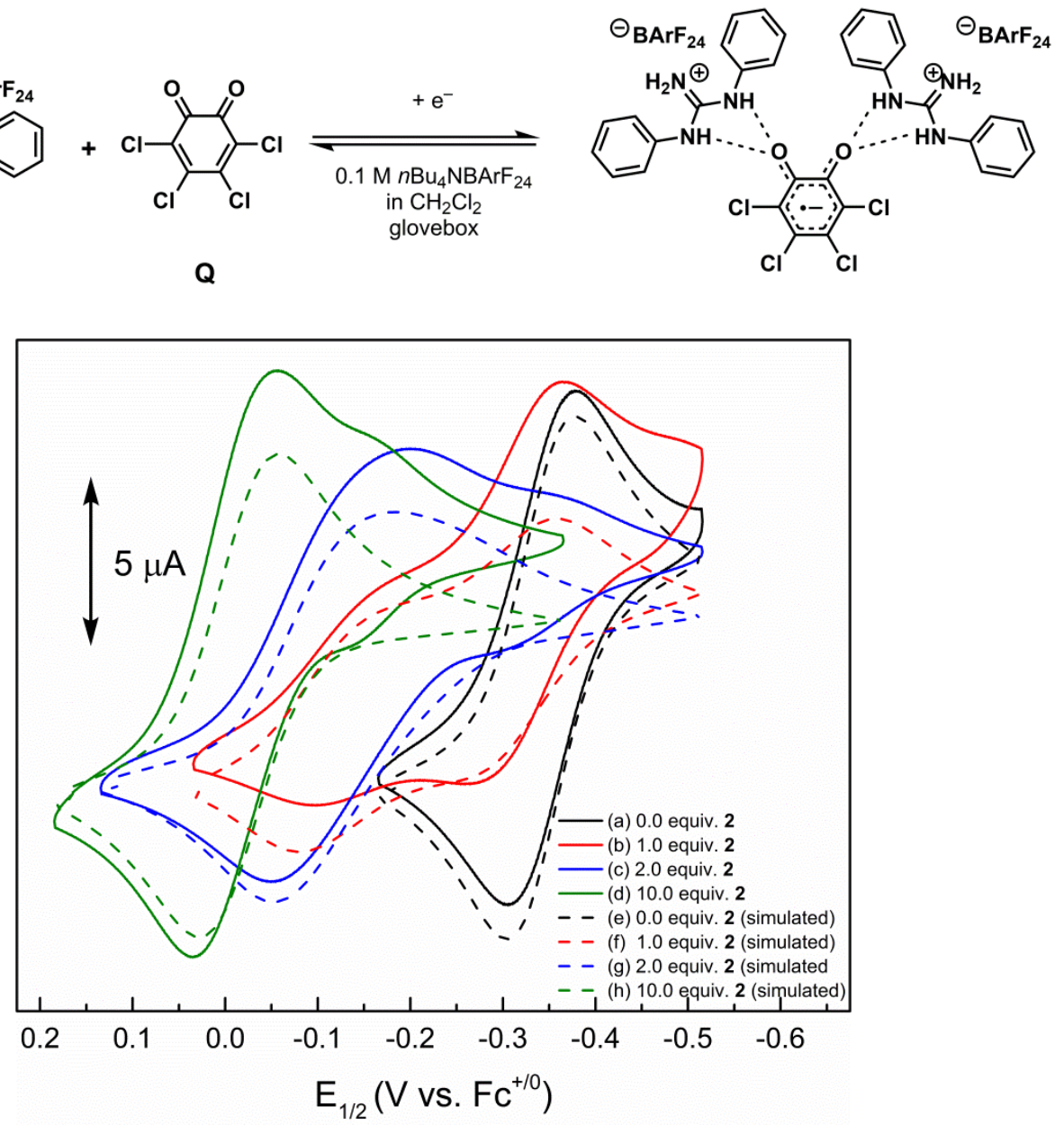

Figure 2.13. Experimental CVs of $o$-chloranil (initial concentration $=0.5 \mathrm{mM}$ ) recorded in 0.1 $\mathrm{M} n \mathrm{Bu}_{4} \mathrm{NBArF}_{24} / \mathrm{CH}_{2} \mathrm{Cl}_{2}$ (glovebox) in the presence of increasing [2] and comparison with simulation of a CEC mechanism. In scans (c) and (d), the additional current peaks at lower potential are attributed to the $2 \mathrm{e}^{-}$reduction. 


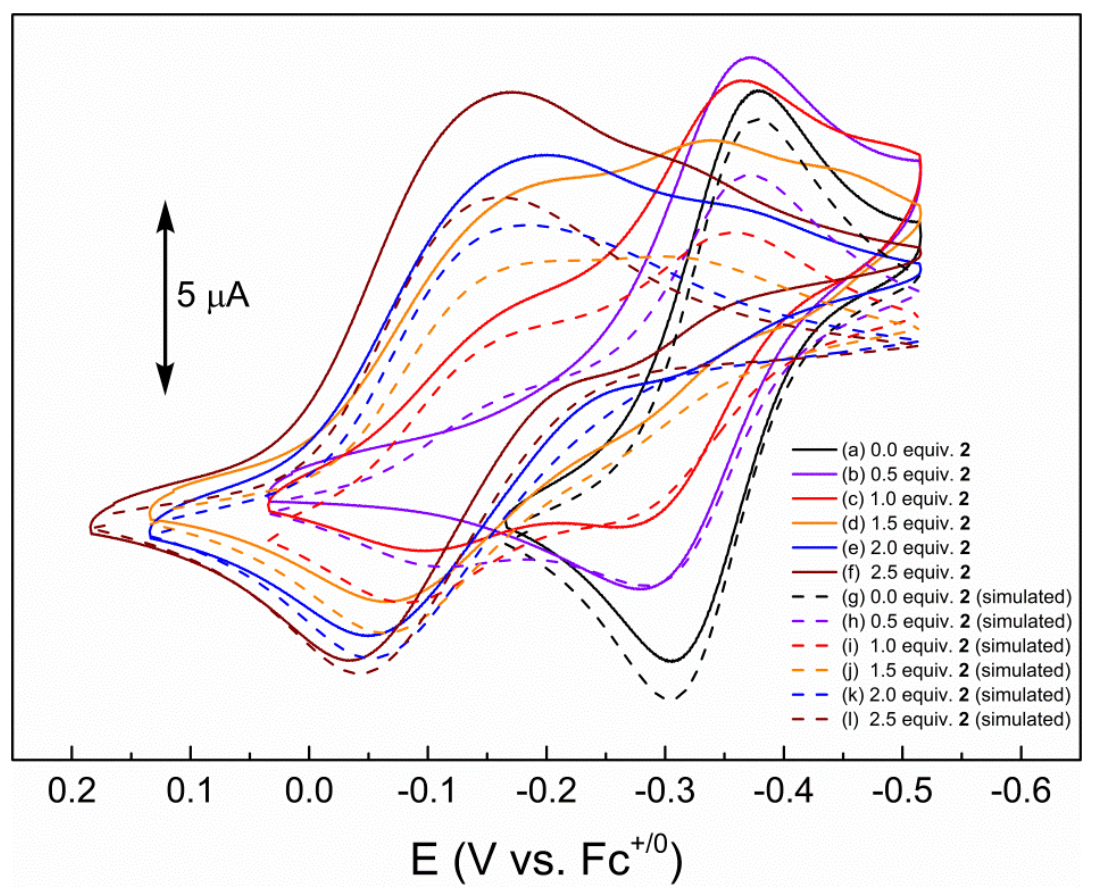

Figure 2.14. Experimental CVs of $o$-chloranil (initial concentration $=0.5 \mathrm{mM}$ ) recorded in 0.1 $\mathrm{M} n \mathrm{Bu}_{4} \mathrm{NBArF}_{24} / \mathrm{CH}_{2} \mathrm{Cl}_{2}$ (glovebox) in the presence of increasing [2] and comparison with simulation of a CEC mechanism. Only data collected at low [2] are shown.

As is the case with $\mathbf{1}$, the data recorded in the presence of $\mathbf{2}$ are best simulated by a CEC mechanism, which replicates both the overall $\Delta \mathrm{E}_{1 / 2}$ and the distinct curve shapes at low [2]. Computations on this 2:1 complex also show bifurcated H-bonding interactions with distances of $1.81 \AA$ and $1.86 \AA$ calculated with B3LYP, and $1.75 \AA$ and $1.89 \AA$ calculated with M06-2X. In the calculation using M06-2X, an apparent slipped pi-stacking interaction between the arenes of the two HBD molecules is observed, with a distance between the arenes of $3.4 \AA$ (Figure 2.15). The value of $\mathrm{K}_{3} \mathrm{~K}_{4}$ describing formation of this $(2)_{2} \cdot \mathbf{Q}^{\bullet-}$ complex was calculated to be $1.8 \times 10^{10}$ $\mathrm{M}^{-2}$, using the parameters obtained from the simulation (Table 2.2). 

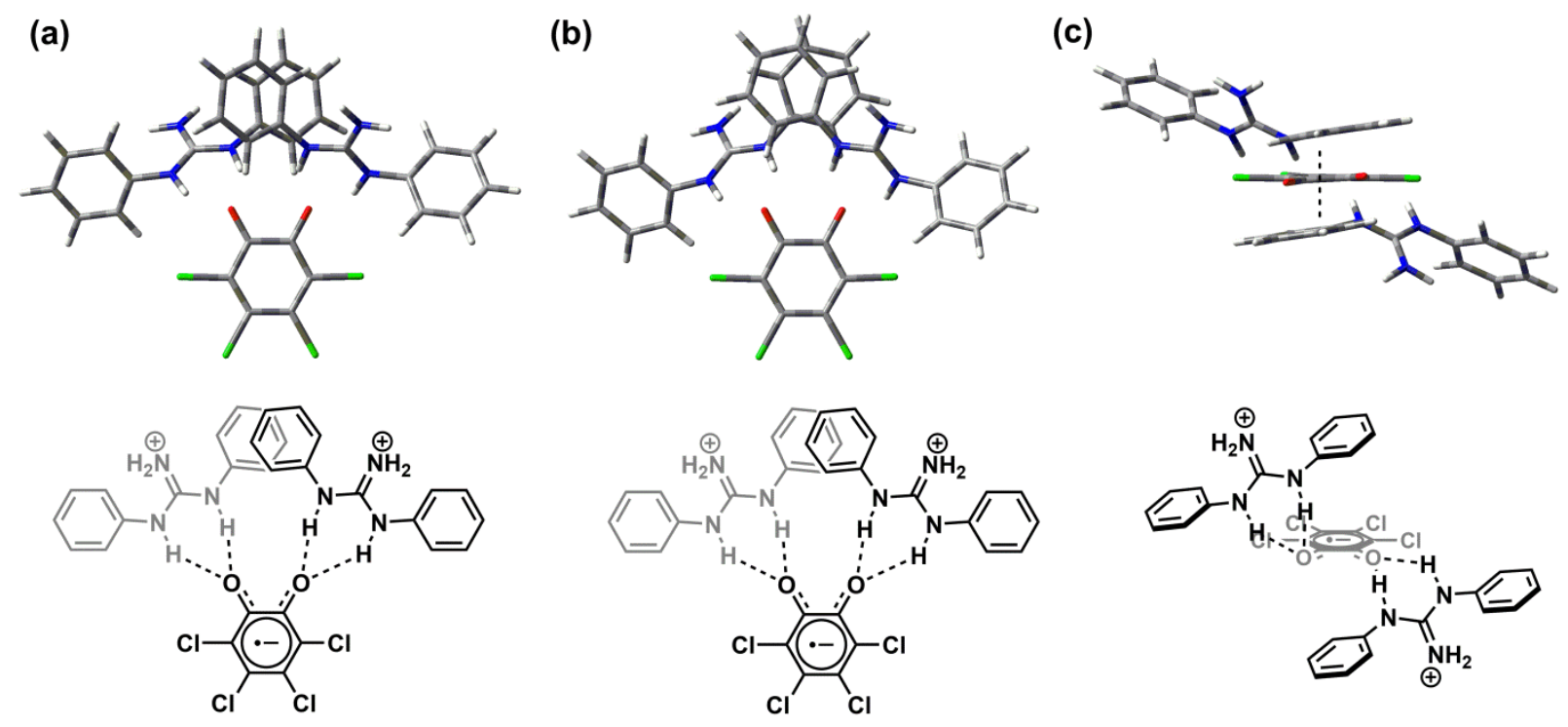

Figure 2.15. Geometry calculated for the $(\mathbf{2})_{2} \cdot \mathbf{Q}^{\bullet-}$ complex, optimized using (a) B3LYP/6$31 \mathrm{G}(\mathrm{d}, \mathrm{p})$ level of theory; the gray HBD molecule is oriented behind the other HBD; (b) M062X/6-31G(d,p) level of theory; (c) Pi-stacking interaction between the arenes on the HBD.

Table 2.2. Equations and best-fit parameters used to simulate $\mathrm{CV}$ data for $0.5 \mathrm{mM}$-chloranil (Q) in $0.1 \mathrm{M} \mathrm{nBu} \mathrm{NBArF}_{24} / \mathrm{CH}_{2} \mathrm{Cl}_{2}$ (glovebox) in the presence of increasing [2]. Italicized values were fixed in the simulation.

\begin{tabular}{cccc}
\hline $\begin{array}{c}\text { Electron transfer } \\
\text { steps }\end{array}$ & $\mathbf{E}_{\mathbf{1} / \mathbf{2}}\left(\mathbf{V} \mathbf{~ v s .} \mathbf{F c} \mathbf{c}^{\mathbf{0}+}\right)$ & $\boldsymbol{\alpha}$ & $\mathbf{k}_{\mathbf{s}}(\mathbf{c m} / \mathbf{s})$ \\
\hline $\mathbf{Q}+\mathrm{e}^{-} \rightarrow \mathbf{Q}^{\bullet-}$ & -0.34 & 0.5 & 0.022 \\
$\mathbf{2} \cdot \mathbf{Q}+\mathrm{e}^{-} \rightarrow \mathbf{2} \cdot \mathbf{Q}^{\bullet-}$ & -0.056 & 0.5 & 1.5 \\
\hline $\mathbf{C h e m i c a l ~ s t e p s}$ & $\mathbf{K}_{\mathrm{eq}}\left(\mathbf{M}^{-\mathbf{1}}\right)$ & $\mathbf{k}_{\mathbf{f}}\left(\mathbf{s}^{-\mathbf{1}}\right)$ \\
\hline $\mathbf{2}+\mathbf{Q} \rightarrow \mathbf{2} \cdot \mathbf{Q}$ & 5.5 & $1 \times 10^{10}$ \\
$\mathbf{2}+\mathbf{2} \cdot \mathbf{Q}^{\bullet-} \rightarrow(\mathbf{2})_{\mathbf{2}} \cdot \mathbf{Q}^{\bullet-}$ & $5.2 \times 10^{4}$ & $1 \times 10^{10}$
\end{tabular}




\section{2a.5.3 Mechanistic analysis of HBD-coupled electron transfer using neutral ureas}

Titration of $o$-chloranil with urea 3 affords the CV traces shown in Figure 2.16. The same features observed at low [1] and [2] are also present at low [3], although the effect is significantly less pronounced. A CEC mechanism again provides the best fit to the experimental data, demonstrating that in the case of neutral, dual HBDs, a 2:1 complex is formed with the reduced quinone. The parameters and equations used to simulate the titrations with $\mathbf{3}$ are summarized in Table 2.3. Analysis of the best-fit parameters yields a $\mathrm{K}_{3} \mathrm{~K}_{4}$ value of $1.0 \times 10^{7} \mathrm{M}^{-2}$ for formation of $(3)_{2} \cdot \mathbf{Q}^{\bullet-}$.<smiles>CCOC(=O)c1cc(NC(=O)Nc2cc(C(=O)OCC)cc(C(F)(F)F)c2)cc(C(F)(F)F)c1</smiles>

3

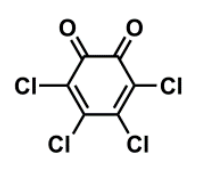

Q

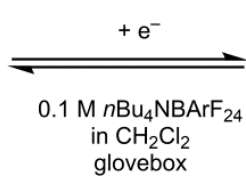

glovebox<smiles>CCOC(=O)c1cc(NC(=O)c2cc(C(=O)OCC)cc(C(F)(F)F)c2)cc(NC(=O)c2cc(C(=O)OCC)cc(C(F)(F)F)c2)c1</smiles>

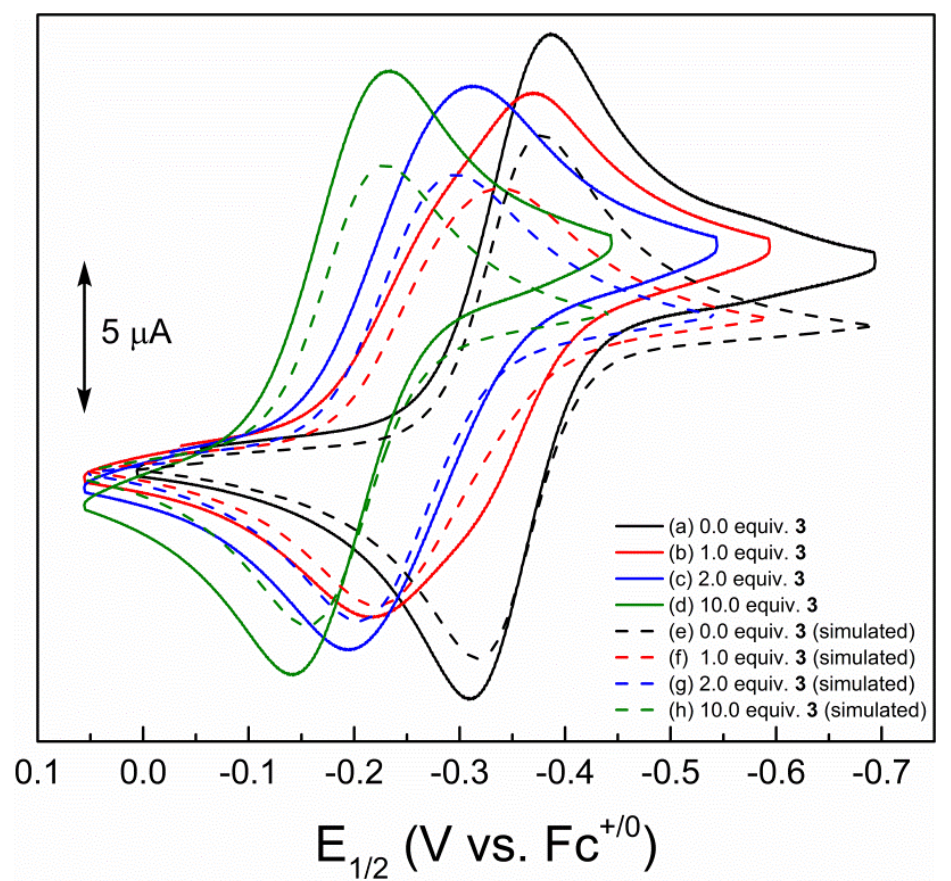

Figure 2.16. Experimental CVs of $o$-chloranil (initial concentration $=0.5 \mathrm{mM}$ ) recorded in 0.1 $\mathrm{M} n \mathrm{Bu}_{4} \mathrm{NBArF}_{24} / \mathrm{CH}_{2} \mathrm{Cl}_{2}$ (glovebox) in the presence of increasing [3] and comparison with simulation of a CEC mechanism. 
Table 2.3. Equations and best-fit parameters used to simulate $\mathrm{CV}$ data for $0.5 \mathrm{mM}$ o-chloranil (Q) in $0.1 \mathrm{M} \mathrm{nBu} \mathrm{NBArF}_{24} / \mathrm{CH}_{2} \mathrm{Cl}_{2}$ (glovebox) in the presence of increasing [3]. Italicized values were fixed in the simulation.

\begin{tabular}{cccc}
\hline $\begin{array}{c}\text { Electron transfer } \\
\text { steps }\end{array}$ & $\mathbf{E}_{\mathbf{1} / \mathbf{2}}\left(\mathbf{V} \mathbf{v s .} \mathbf{F c}^{\mathbf{0 / +}}\right)$ & $\boldsymbol{\alpha}$ & $\mathbf{k}_{\mathbf{s}}(\mathbf{c m} / \mathbf{s})$ \\
\hline $\mathbf{Q}+\mathrm{e}^{-} \rightarrow \mathbf{Q}^{\bullet-}$ & -0.35 & 0.5 & 0.022 \\
$\mathbf{3} \cdot \mathbf{Q}+\mathrm{e}^{-} \rightarrow \mathbf{3} \cdot \mathbf{Q}^{\bullet-}$ & -0.098 & 0.5 & 0.29 \\
\hline $\mathbf{C h e m i c a l ~ s t e p s}$ & $\mathbf{K}_{\mathbf{e q}}\left(\mathbf{M}^{-\mathbf{1}}\right)$ & & $1 \times 10^{10}$ \\
\hline $\mathbf{3}+\mathbf{Q} \rightarrow \mathbf{3} \cdot \mathbf{Q}$ & 3.5 & $1 \times 10^{10}$
\end{tabular}

\section{2a.5.4 Comparison of binding abilities for HBDs 1-3}

Table 2.4 summarizes the data obtained from the simulations with HBDs $\mathbf{1}-\mathbf{3}$. All three HBDs modulate the apparent potential of an electron-deficient quinone to an extent greater than that observed in any previous attempt to do so. ${ }^{\text {a,b,d }}$ Diphenylguanidinium 2 offers the greatest degree of stabilization to $\mathbf{Q}^{\bullet-}$ and urea $\mathbf{3}$ offers the weakest, with a difference of three orders of magnitude between them. A comparison of these values provides insight into the ways in which the nature of the HBD can influence its interaction with $\mathbf{Q}^{\bullet-}$. The difference between $\mathbf{1}$ and $\mathbf{2}$ can be ascribed to a difference in acidity - the $\mathrm{pK}_{\mathrm{a}}$ values in DMSO for an $N, N^{\prime}$-dialkylguanidinium ion and 2 are 14.1 and 10.1 , respectively. ${ }^{10}$ Such an effect implicates hydrogen bonding interactions in the modulation of $\Delta \mathrm{G}_{\text {bind }}$ and increased favorability of electron transfer to 2 . On the other hand, neutral $3\left(\mathrm{pK}_{\mathrm{a}}=13.8\right.$ in DMSO) ${ }^{11}$ and cationic $\mathbf{1}$ have similar $\mathrm{pK}_{\mathrm{a}}$ values, yet $\mathrm{K}_{3} \mathrm{~K}_{4}$ for 3 is weaker by an order of magnitude. This result demonstrates the importance of

\footnotetext{
${ }^{10}$ Uyeda, C.H. Catalysis of the Claisen Rearrangement by Hydrogen Bond Donors. Ph.D. Thesis, Harvard University, Cambridge, MA 2010

${ }^{11}$ Jakab, F.; Tancon, C.; Zhang, Z.; Lippert, K. M.; Schreiner, P. R. Org. Lett. 2012, 14, 1724-1727
} 
electrostatic effects in HBD-coupled electron transfer. Taken together, these trends lead us to conclude that both H-bonding and electrostatic effects play a crucial role in HBD- coupled electron transfer.

Table 2.4. Summary of parameters for HBD-coupled electron transfer promoted by HBDs 1-3.

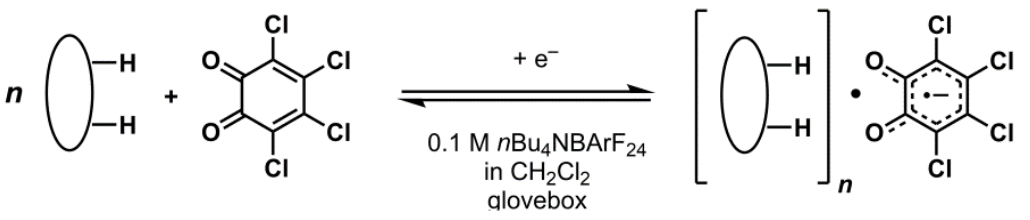

\begin{tabular}{ccccc}
\hline HBD & $\mathrm{K}_{1}\left(\mathrm{M}^{-1}\right)$ & $\mathrm{E}_{2}(\mathrm{~V})$ & $\mathrm{K}_{3}\left(\mathrm{M}^{-1}\right)^{\mathrm{b}}$ & $\mathrm{K}_{3} \mathrm{~K}_{4}\left(\mathrm{M}^{-2}\right)$ \\
\hline $\mathbf{1}$ & 66 & -0.17 & $\left(3.4 \times 10^{4}\right)$ & $6.1 \times 10^{8}$ \\
$\mathbf{2}$ & 5.5 & -0.057 & $\left(3.5 \times 10^{5}\right)$ & $1.8 \times 10^{10}$ \\
$\mathbf{3}$ & 3.5 & -0.098 & $\left(5.6 \times 10^{4}\right)$ & $1.0 \times 10^{7}$
\end{tabular}

${ }^{a}$ Parameters were determined by titrating $0.5 \mathrm{mM} \mathrm{Q}$ in $0.1 \mathrm{M} n \mathrm{Bu}_{4} \mathrm{NBArF}_{24} / \mathrm{CH}_{2} \mathrm{Cl}_{2}$ (glovebox) with $[\mathrm{HBD}]$ and simulating the experimental CVs obtained. ${ }^{b}$ Values in parentheses are defined by other parameters simulated in the square scheme.

These results, combined with the fact that each HBD binds $\mathbf{Q}^{\bullet-}$ with 2:1 stoichiometry, also have important implications for the HBD-coupled electron transfer strategy. The improved binding of $\mathbf{2}$ relative to the other HBDs clearly indicates that most substantial stabilization of the reduced state is achieved when a dicationic complex involving two HBDs is formed. 
2a.5.5 Mechanistic analysis of HBD-coupled electron transfer using dicationic bisamidinium salts

The observation that HBDs 1-3 all bind $\mathbf{Q}^{\bullet-}$ in 2:1 complexes, with HBD charge playing a critical role, prompted us to examine bis-amidinium salt $\mathbf{4}$, which involves a covalent linkage between two cationic subunits (Figure 2.17).

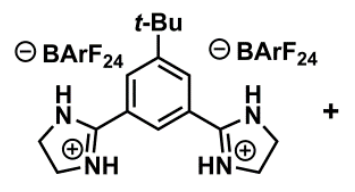

4<smiles>O=C1C(=O)C(Cl)=C(Cl)C(Cl)=C1Cl</smiles>

Q

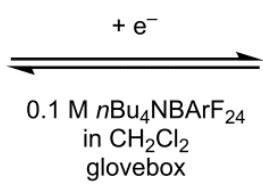

glovebox
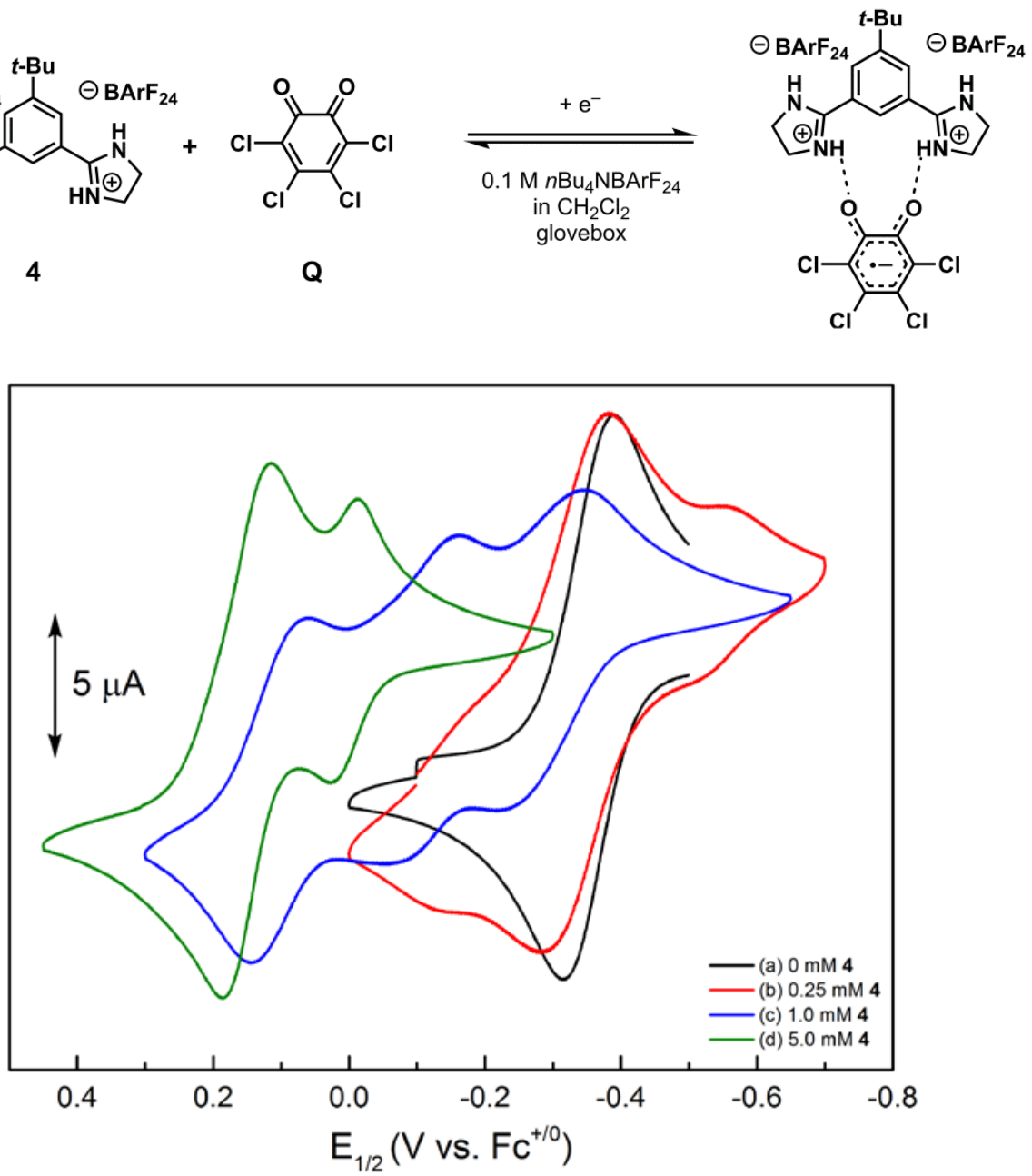

Figure 2.17. Experimental CVs of $o$-chloranil (initial concentration $=0.5 \mathrm{mM}$ ) recorded in 0.1 $\mathrm{M} n \mathrm{Bu}_{4} \mathrm{NBArF}_{24} / \mathrm{CH}_{2} \mathrm{Cl}_{2}$ (glovebox) in the presence of increasing [4]. In scans (b)-(d), the additional current peaks at lower potential are attributed to the $2 \mathrm{e}^{-}$reduction.

Qualitative differences are evident between the CVs recorded with 4 and the CVs discussed above. As in the CVs recorded with $\mathbf{1 - 3}$, an increase in current at high potential is 
observed at the expense of current magnitude at low potential. This indicates that the mechanism for HBD-coupled electron transfer promoted by 4 still involves pre-association between the HBD and the neutral quinone. With $\mathbf{4}$, however, the increase in current occurs at a much higher potential $(0.1 \mathrm{~V})$, and very little shift in apparent potential is observed upon further addition of HBD. As with $\mathbf{2}$, the $\mathrm{CV}$ wave corresponding to the $\mathbf{Q}^{\bullet-} / \mathbf{Q}^{2-}$ redox couple undergoes a very large shift upon addition of 4 . In scans (c) and (d), the current peaks at low potential are attributed to this electron transfer event, which was not included in the simulations. Additionally, a current peak between the peaks corresponding to the first- and second-electron transfers is also observed in scan (c).

The simulations reveal that a shift in mechanism occurs when $\mathbf{4}$ is used. Unlike the other HBDs, $\mathbf{4}$ binds to $\mathbf{Q}^{\bullet-}$ with 1:1 stoichiometry, and a CE mechanism-a single binding step $\left(\mathrm{K}_{1}\right)$ followed by electron transfer step $\left(\mathrm{E}_{2}\right)$ (Figure 2.18) - best describes the experimental data. These results are consistent with our observation that two monocationic HBDs are required to provide significant thermodynamic stabilization to the reduced state in a dicatonic complex. A single dicationic $\mathrm{HBD}$ can also bind tightly to $\mathbf{Q}^{\bullet-}$, and by tethering the cationic subunits together, a greater degree of stabilization can be achieved. Computations of a $b i s$-amidinium $\cdot \mathbf{Q}^{\bullet-}$ complex (abbreviated to remove the $t$-butyl substituent) indicate that the binding geometry involves a single H-bond between the HBD and each oxygen of the quinone (Figure 2.19). In the calculations using B3LYP, both H-bonds are $1.57 \AA$ in length, although the structures indicate that the binding orientation may not be symmetric. 


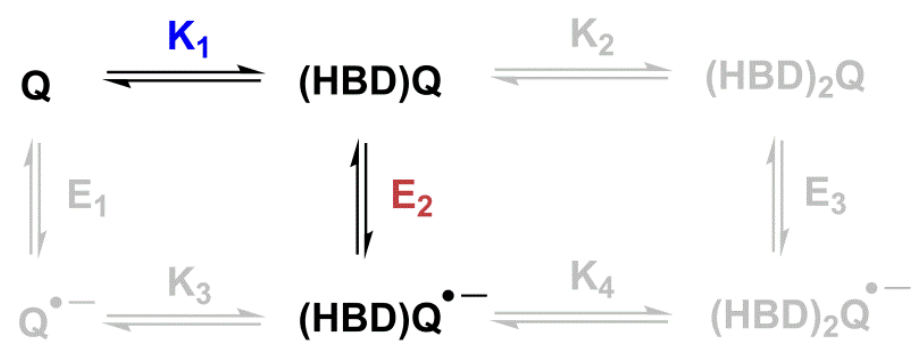

Figure 2.18. CE mechanism.
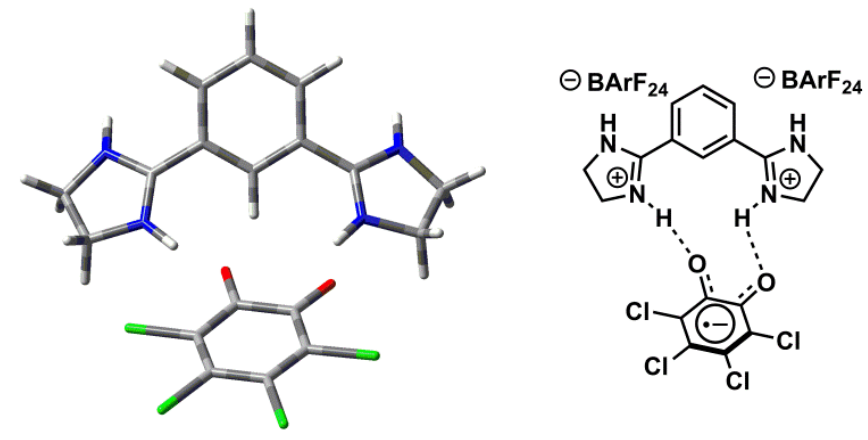

Figure 2.19. Geometry calculated for the bis-amidinium $\cdot \mathbf{Q}^{--}$complex, optimized using the B3LYP/6-31G(d,p) level of theory.

A comparison of the experimental data and the simulations that result from a $\mathrm{CE}$ mechanism are shown in Figure 2.20. Although the current that appears between the two waves corresponding to the first- and second-electron reductions is not reproduced by a CE mechanism, other features are appropriately reproduced. The CE mechanism does fit the overall $\Delta \mathrm{E}_{1 / 2}$ as [4] increases, with good overlap between the wave at high potential in scan (d) and simulated scan (h). The reversibility in the CV waves over the course of the titration is also replicated in the simulation.

Attempts to find equations and corresponding parameters that explain the appearance of the middle wave, while still adequately representing the experimental data as a whole, were unsuccessful. Simulations of an EC mechanism fail to provide reversible CV traces as observed 
in the experimental data. A CEC mechanism, which provides the best fit in the CV experiments using HBDs 1-3, does provide a suitable fit to the experimental data with respect to the overall $\Delta \mathrm{E}_{1 / 2}$, but offers no improvement over the $\mathrm{CE}$ mechanism. Consideration of the Coulombic repulsion present in a 2:1 complex involving dicatonic HBDs—in a tetracationic complex-leads us to reject the CEC mechanism as a reasonable description of these data. Furthermore, evidence in support of a 1:1 complex was obtained independently (vide infra), while no additional support for a 2:1 complex has been found.

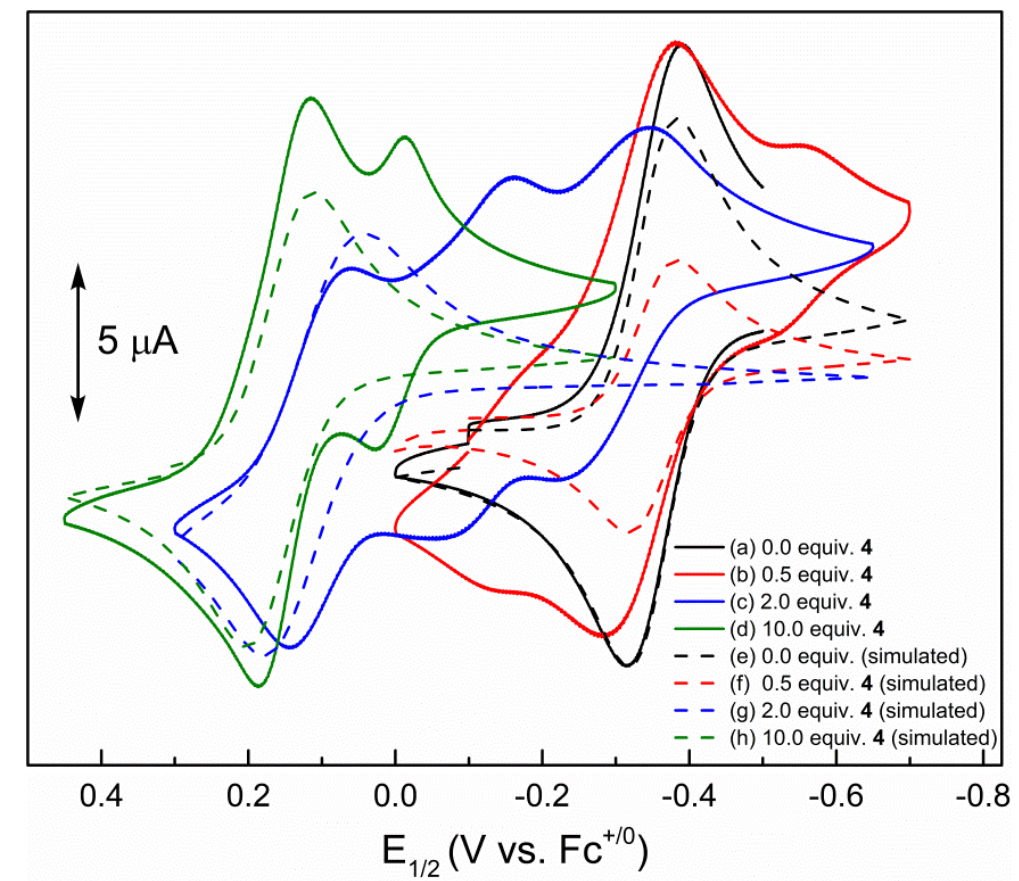

Figure 2.20. Experimental CVs of $o$-chloranil (initial concentration $=0.5 \mathrm{mM}$ ) recorded in 0.1 $\mathrm{M} n \mathrm{Bu}_{4} \mathrm{NBArF}_{24} / \mathrm{CH}_{2} \mathrm{Cl}_{2}$ (glovebox) in the presence of increasing [4] and comparison with simulation of a $\mathrm{CE}$ mechanism. In scans (b)-(d), the additional current peaks at lower potential are attributed to the $2 \mathrm{e}^{-}$reduction.

An explanation for the extra current may be found in the literature, as similar phenomena have been observed in other cyclic voltammetry experiments using quinones. In studies of both $p$ - and $o$-quinones, as well as related quinone-like structures, the formation of $\sigma$-quinone dimers 
involving a covalent linkage and $\pi$-dimers associated through noncovalent interactions has been offered as an explanation for extra current in CVs of these molecules. ${ }^{12}$ Smith and coworkers have investigated this issue in detail, and present a more likely possibility for the appearance of current between the waves corresponding to the first- and second-electron reductions. ${ }^{13}$ Their work determined that formation of oxidized functional groups on the glassy carbon surface can interact with the quinone and lead to formation of this extra current. The effects observed in this report are strikingly similar to those observed in our titrations using $\mathbf{4}$, and these oxidized functional groups may be present under our experimental conditions. The appearance of this extra current seems to be related to the nature of the HBD, as it does not appear in every case. Perhaps an interaction between acidic HBD 4 and the electrode surface provides an additional pathway for quinone reduction that affords the extra current observed. Such a pathway, however, would not interfere with the intrinsic ability of $\mathbf{4}$ to facilitate electron transfer to $\mathbf{Q}$.

\section{2a.5.6 Comparison of bis-amidinium salt 4 with HBDs 1-3}

The parameters and equations used to simulate the titrations with $\mathbf{4}$ are summarized in Table 2.5. This analysis reveals that the value for $K_{3}$ describing the formation of $\mathbf{4} \cdot \mathbf{Q}^{\bullet-}$ is $9.2 \times 10^{10} \mathrm{M}^{-1}$. Due to the change in stoichiometry when $\mathbf{4}$ is used, the values for $\mathrm{K}_{3}$ must be compared in order to evaluate the efficacy of 4 relative to the other HBDs; these values are summarized in Table 2.6.

A comparison of these values shows that $\mathbf{4}$ is exceptionally effective at promoting electron transfer and is six orders of magnitude more potent than $\mathbf{2}$ at binding $\mathbf{Q}^{\bullet-}$. This strength

${ }^{12}$ (a) Macías-Ruvalcaba, N. A.; Felton, G. A. N.; Evans, D. H. J. Phys. Chem. C. 2009, 113, 338-345. (b) Macías-Ruvalcaba, N. A.; Evans, D. H. J. Phys. Chem. C. 2010, 114, 1285-1292. (c) René A.; Evans, D. H. J. Phys. Chem. C. 2012, 116, 14454-14460. (c)

${ }^{13}$ Staley, P. A.; Newell, C. M.; Pullman, D. P.; Smith, D. K. Anal. Chem. 2014, 86, 10917-10924. 
of binding offers a stark contrast to literature precedent examining electron-deficient quinones, in which no measurable effect of H-bonding was observed on the initial electron transfer. Furthermore, reversibility in the CVs is maintained over the course of the titration, indicating that the $\mathbf{4} \cdot \mathbf{Q}^{\bullet-}$ complex is stable under the experimental conditions and does not experience full proton transfer or other decomposition pathways.

Table 2.5. Equations and parameters used to simulate $\mathrm{CV}$ data for $0.5 \mathrm{mM} o$-chloranil $(\mathbf{Q})$ in $0.1 \mathrm{M} \mathrm{nBu} \mathrm{Bu}_{4} \mathrm{NBArF}_{24} / \mathrm{CH}_{2} \mathrm{Cl}_{2}$ (glovebox) in the presence of increasing [4]. Italicized values were fixed in the simulation.

\begin{tabular}{cccc}
\hline $\begin{array}{c}\text { Electron transfer } \\
\text { steps }\end{array}$ & $\mathbf{E}_{\mathbf{1 / 2}}\left(\mathbf{V} \mathbf{v s} . \mathbf{F c}^{+/ 0}\right)$ & $\boldsymbol{\alpha}$ & $\mathbf{k}_{\mathbf{s}}(\mathbf{c m} / \mathbf{s})$ \\
\hline $\mathbf{Q}+\mathrm{e}^{-} \rightarrow \mathbf{Q}^{--}$ & -0.35 & 0.5 & 0.022 \\
$\mathbf{4} \cdot \mathbf{Q}+\mathrm{e}^{-} \rightarrow \mathbf{4} \cdot \mathbf{Q}^{\cdot-}$ & 0.28 & 0.5 & 0.10 \\
\hline $\mathbf{C h e m i c a l ~ s t e p s}$ & $\mathbf{K}_{\mathbf{e q}}\left(\mathbf{M}^{-1}\right)$ & & $\mathbf{k}_{\mathbf{f}}\left(\mathbf{s}^{-1}\right)$ \\
\hline $\mathbf{4}+\mathbf{Q} \rightarrow \mathbf{4} \cdot \mathbf{Q}$ & 2 & & $1 \times 10^{10}$
\end{tabular}


Table 2.6. Summary of parameters for HBD-coupled electron transfer promoted by HBDs $\mathbf{1}-\mathbf{4}$ and additive 5.

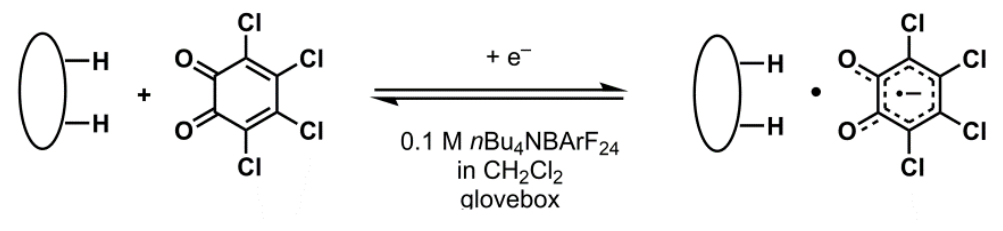

\begin{tabular}{ccc}
\hline HBD & $\mathrm{E}_{2}(\mathrm{~V})$ & $\mathrm{K}_{3}\left(\mathrm{M}^{-1}\right)^{\mathrm{b}}$ \\
\hline $\mathbf{1}$ & -0.166 & $\left(3.4 \times 10^{4}\right)$ \\
$\mathbf{2}$ & -0.057 & $\left(3.5 \times 10^{5}\right)$ \\
$\mathbf{3}$ & -0.098 & $\left(5.6 \times 10^{4}\right)$ \\
$\mathbf{4}$ & 0.280 & $9.2 \times 10^{10}$
\end{tabular}

${ }^{a}$ Parameters were determined by titrating $0.5 \mathrm{mM} \mathrm{Q}$ in $0.1 \mathrm{M} n \mathrm{Bu}_{4} \mathrm{NBArF}_{24} / \mathrm{CH}_{2} \mathrm{Cl}_{2}$ (glovebox) with [HBD] and simulating the experimental CVs obtained. ${ }^{b}$ Values in parentheses are defined by other parameters simulated in the square scheme.

\section{2a.5.7 Control experiments and comparison with bis-amidinium salt 4}

To probe the interplay of hydrogen bonding and electrostatic interactions in stabilizing $\mathbf{Q}^{\bullet-}$, tetramethylated bis-amidinium salt 5-which bears the same net charge as $\mathbf{4}$ but lacks the ability to form H-bonds-was examined. To probe the effect of tethering more directly, amidinium 6, which is a monomeric subunit of $\mathbf{4}$, was also studied.

The CVs recorded with 5 are shown in Figure 2.21, and the equations and parameters used to simulate these data are summarized in Table 2.7. The additional current that appears at lower potential in scans (b)-(c) is attributed to an interaction between $\mathbf{5}$ and the quinone dianion, which was not included in the simulations. An EC pathway, in which electron transfer $\left(\mathrm{E}_{1}\right)$ precedes binding to the $\operatorname{HBD}\left(\mathrm{K}_{3}\right)$, is found to best represent the experimental data (Figure 2.22). This change in mechanism establishes that hydrogen bonding is necessary for pre-association 
between $\mathbf{Q}$ and the HBD, and thereby dictates the pathway by which HBD-coupled electron transfer occurs. The sensitivity of the simulations to this mechanistic change also lends credence to our mechanistic analysis in general. The fact that 5-the only non-hydrogen bonding additive - is the only small molecule to promote an EC mechanism validates our conclusion that HBDs bind to the neutral quinone prior to the electron transfer event. This result also explicitly illustrates that hydrogen bonding is required for pre-association between the HBD and the quinone.

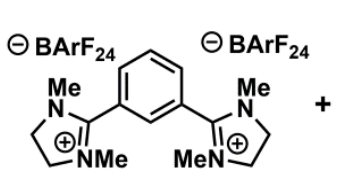

5<smiles>O=C1C(=O)C(Cl)=C(Cl)C(Cl)=C1Cl</smiles>

$\mathbf{0}$

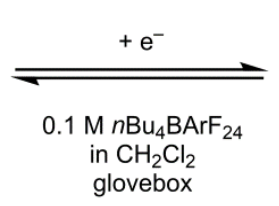

glovebox

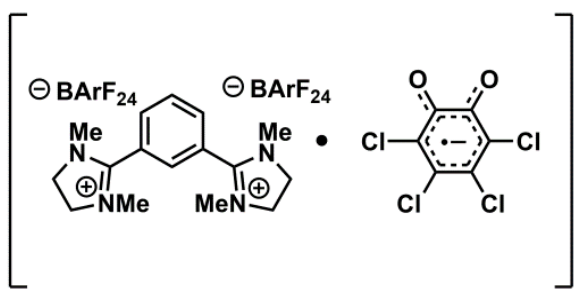

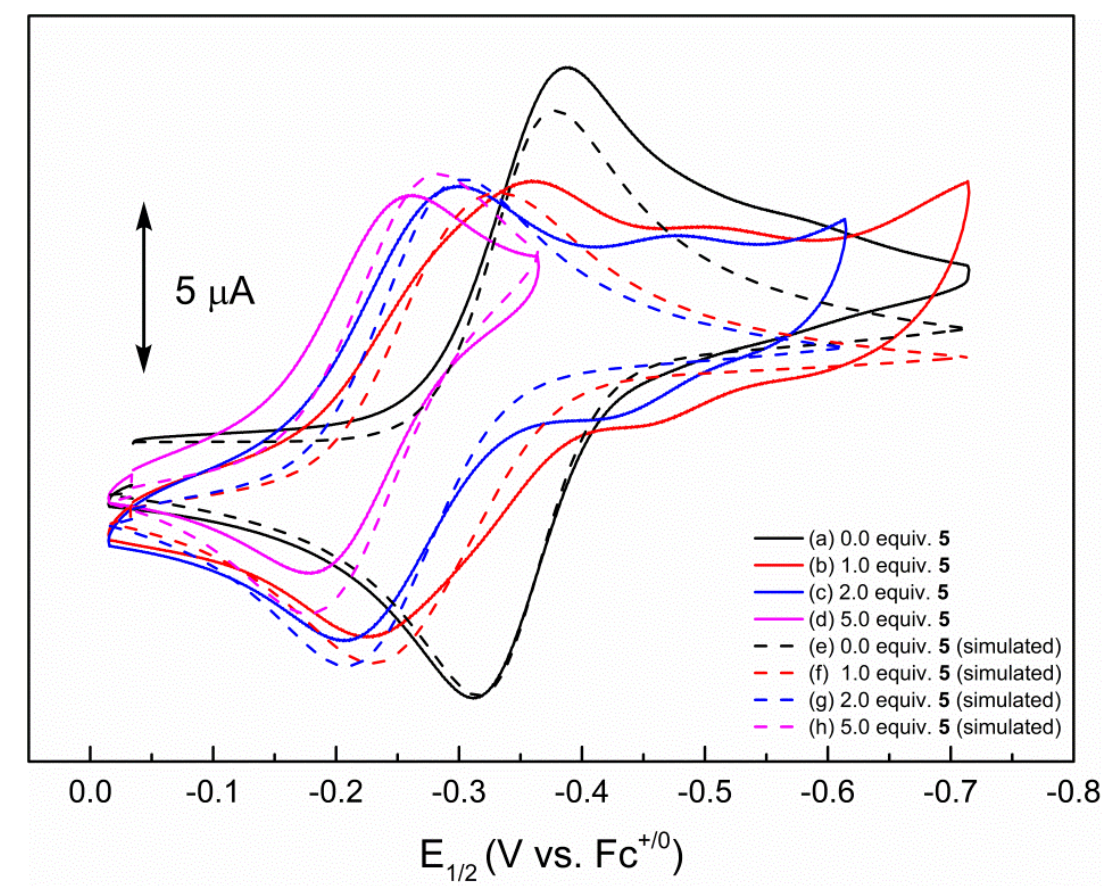

Figure 2.21. Experimental CVs of $o$-chloranil (initial concentration $=0.5 \mathrm{mM}$ ) recorded in 0.1 $\mathrm{M} n \mathrm{Bu}_{4} \mathrm{NBArF}_{24} / \mathrm{CH}_{2} \mathrm{Cl}_{2}$ (glovebox) in the presence of increasing [5] and comparison with simulation of an EC mechanism. In scans (b) and (c), the additional current peaks at lower potential are attributed to the $2 \mathrm{e}^{-}$reduction. 
Table 2.7. Equations and best-fit parameters used to simulate $\mathrm{CV}$ data for $0.5 \mathrm{mM}$ o-chloranil (Q) in $0.1 \mathrm{M} \mathrm{nBu} 4 \mathrm{NBArF}_{24} / \mathrm{CH}_{2} \mathrm{Cl}_{2}$ (glovebox) in the presence of increasing [5]. Italicized values were fixed in the simulation.

\begin{tabular}{cccc}
\hline $\begin{array}{c}\text { Electron transfer } \\
\text { steps }\end{array}$ & $\mathbf{E}_{\mathbf{1 / 2}}\left(\mathbf{V}\right.$ vs. Fc $\left.{ }^{0 /+}\right)$ & $\alpha$ & $\mathbf{k}_{\mathbf{s}}(\mathbf{c m} / \mathbf{s})$ \\
\hline $\mathbf{Q}+\mathrm{e}^{-} \rightarrow \mathbf{Q}^{\bullet-}$ & -0.38 & 0.5 & 0.06
\end{tabular}

\begin{tabular}{ccc}
\hline Chemical steps & $\mathbf{K}_{\mathrm{eq}}\left(\mathbf{M}^{-\mathbf{1}}\right)$ & $\mathbf{k}_{\mathbf{f}}\left(\mathbf{s}^{\mathbf{- 1}}\right)$ \\
\hline $\mathbf{5}+\mathbf{Q}^{\bullet-} \rightarrow \mathbf{5} \cdot \mathbf{Q}^{\bullet-}$ & $5.7 \times 10^{4}$ & $1 \times 10^{10}$
\end{tabular}

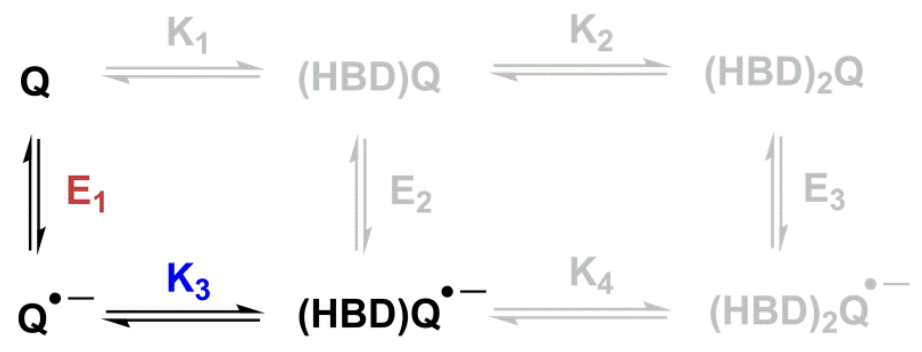

Figure 2.22. EC mechanism.

Amidinium salt $\mathbf{6}$ is also capable of binding to $\mathbf{Q}^{\bullet-}$ and affords shifts in the CV traces recorded for $\mathbf{Q}$ (Figure 2.23). These data also display the features at low [6] (Figure 2.24) that indicate pre-association and are best simulated by a CEC mechanism, consistent with the other monocationic HBDs. Although the discrepancies in current magnitude are large, both the curve shapes obtained at low [6] as well as the overall $\Delta \mathrm{E}_{1 / 2}$ are reproduced. The equations and parameters used to simulate the data collected with $\mathbf{6}$ are summarized in Table 2.8. 


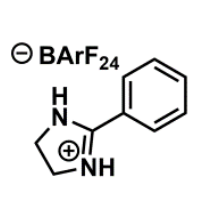

6<smiles>O=C1C(Cl)=C(Cl)C(=O)C(Cl)=C1Cl</smiles>

$\mathbf{Q}$

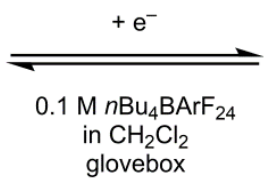

glovebox
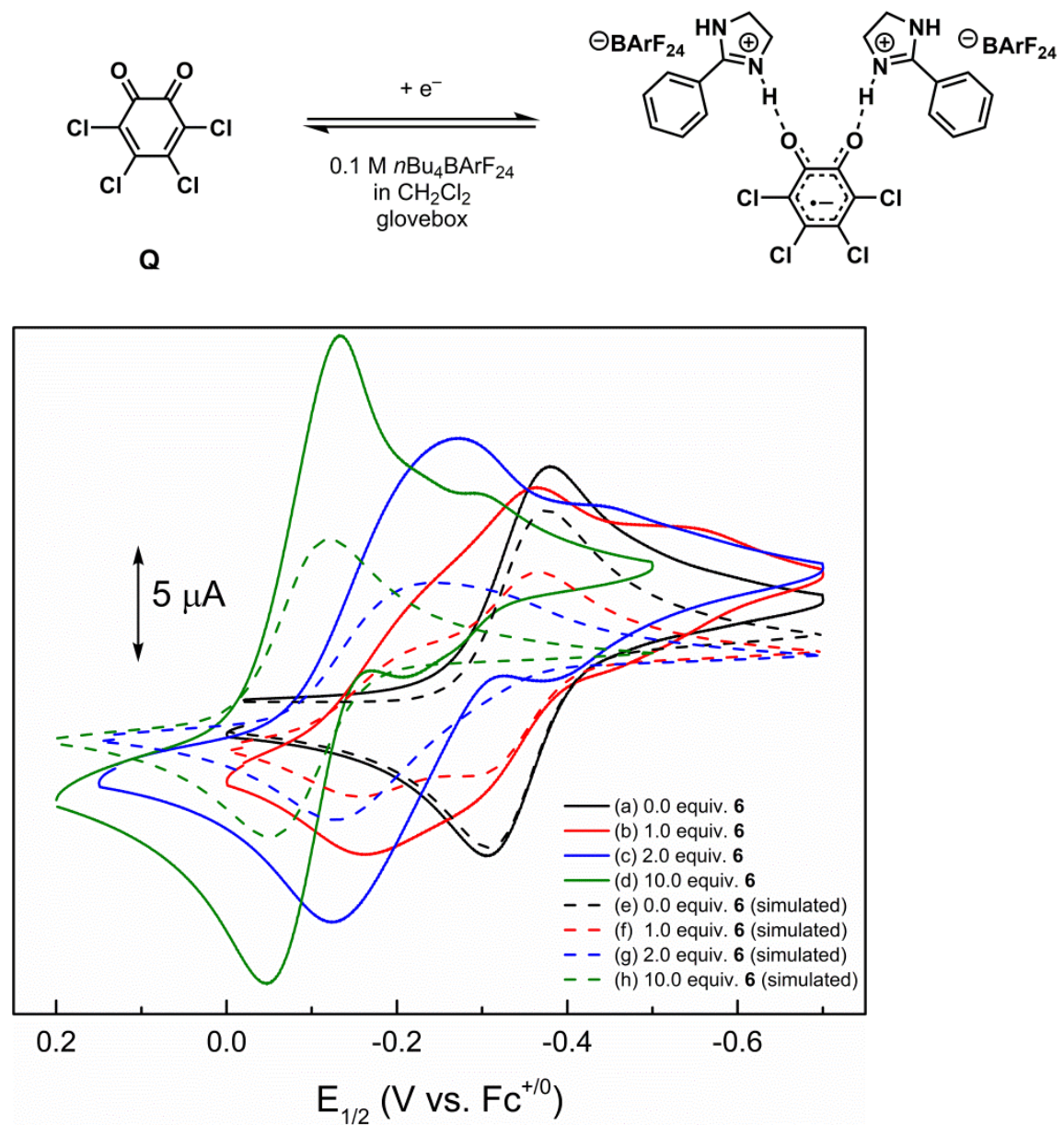

Figure 2.23. Experimental $\mathrm{CVs}$ of $o$-chloranil (initial concentration $=0.5 \mathrm{mM}$ ) recorded in 0.1 $\mathrm{M} n \mathrm{Bu}_{4} \mathrm{NBArF}_{24} / \mathrm{CH}_{2} \mathrm{Cl}_{2}$ (glovebox) in the presence of increasing [6] and comparison with simulation of a CEC mechanism. In scans (b)-(d), the additional current peaks at lower potential are attributed to the $2 \mathrm{e}^{-}$reduction. 


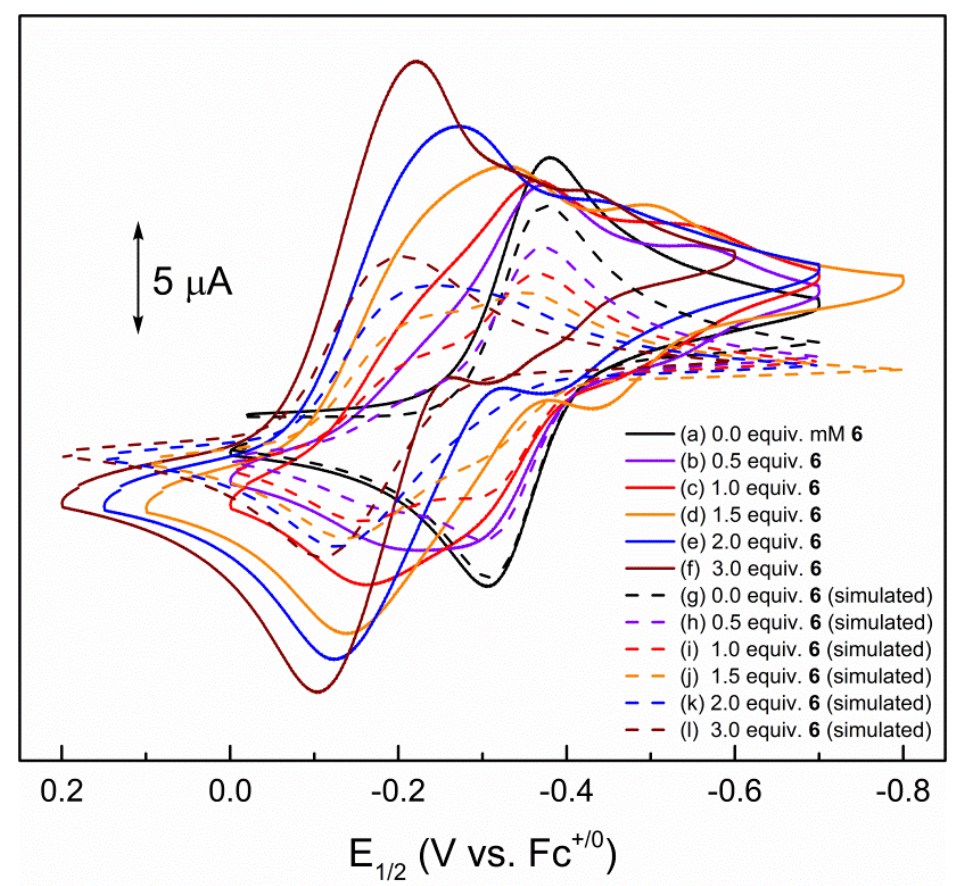

Figure 2.24. Experimental CVs of $o$-chloranil (initial concentration $=0.5 \mathrm{mM}$ ) recorded in 0.1 $\mathrm{M} n \mathrm{Bu}_{4} \mathrm{NBArF}_{24} / \mathrm{CH}_{2} \mathrm{Cl}_{2}$ (glovebox) in the presence of increasing [6] and comparison with simulation of a CEC mechanism. Only data collected at low [6] are shown.

Table 2.8. Equations and best-fit parameters used to simulate $\mathrm{CV}$ data for $0.5 \mathrm{mM} o$-chloranil (Q) in $0.1 \mathrm{M} n \mathrm{Bu}_{4} \mathrm{NBArF}_{24} / \mathrm{CH}_{2} \mathrm{Cl}_{2}$ (glovebox) in the presence of increasing [6]. Italicized values were fixed in the simulation.

\begin{tabular}{cccc}
\hline $\begin{array}{c}\text { Electron transfer } \\
\text { steps }\end{array}$ & $\mathbf{E}_{\mathbf{1} / \mathbf{2}}\left(\mathbf{V}\right.$ vs. Fc $\left.\mathbf{c}^{\mathbf{0 / +}}\right)$ & $\boldsymbol{\alpha}$ & $\mathbf{k}_{\mathbf{s}}(\mathbf{c m} / \mathbf{s})$ \\
\hline $\mathbf{Q}+\mathrm{e}^{-} \rightarrow \mathbf{Q}^{\bullet-}$ & -0.34 & 0.5 & 0.03 \\
$\mathbf{6} \cdot \mathbf{Q}+\mathrm{e}^{-} \rightarrow \mathbf{6} \cdot \mathbf{Q}^{\bullet-}$ & -0.20 & 0.5 & 16 \\
\hline $\mathbf{C h e m i c a l ~ s t e p s}$ & $\mathbf{K}_{\mathrm{eq}}\left(\mathbf{M}^{-\mathbf{1}}\right)$ & $1 \times 10^{10}$ \\
\hline $\mathbf{6}+\mathbf{Q} \rightarrow \mathbf{6} \cdot \mathbf{Q}$ & 50 & $1 \times 10^{10}$
\end{tabular}


A comparison of the values for $\mathrm{K}_{3}$ obtained from the titrations using HBDs 4-6 is shown in Table 2.9. The value for $\mathrm{K}_{3}$ describing formation of $\mathbf{5} \cdot \mathbf{Q}^{\mathbf{}-}$ is six orders of magnitude smaller than that for $\mathbf{4} \cdot \mathbf{Q}^{--}$. While both of these additives are dicationic, only 4 is capable of hydrogen bonding to $\mathbf{Q}^{\bullet-}$. This large difference, therefore, illustrates that the pronounced effect of $\mathbf{4}$ on the stability of the reduced quinone is not purely electrostatic in nature, and that H-bonding interactions are required for effective HBD-coupled electron transfer.

Table 2.9. Summary of parameters for HBD-coupled electron transfer promoted by $4-\mathbf{6} .^{a}$

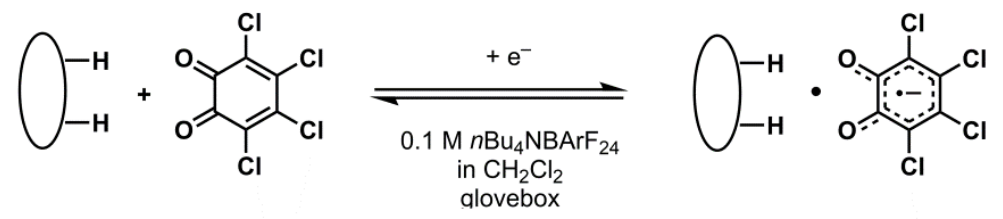

\begin{tabular}{cccc}
\hline HBD & $\mathrm{E}_{2}(\mathrm{~V})$ & $\mathrm{K}_{3}\left(\mathrm{M}^{-1}\right)^{\mathrm{b}}$ & $\mathrm{K}_{3} \mathrm{~K}_{4}\left(\mathrm{M}^{-2}\right)$ \\
\hline $\mathbf{4}$ & 0.28 & $9.2 \times 10^{10}$ & -- \\
$\mathbf{5}$ & -- & $5.7 \times 10^{4}$ & -- \\
$\mathbf{6}$ & -0.20 & $\left(1.2 \times 10^{4}\right)$ & $1.5 \times 10^{9}$
\end{tabular}

${ }^{a}$ Parameters were determined by titrating $0.5 \mathrm{mM} \mathrm{Q}$ in $0.1 \mathrm{M} n \mathrm{Bu}_{4} \mathrm{NBArF}_{24} / \mathrm{CH}_{2} \mathrm{Cl}_{2}$ (glovebox) with [HBD] and simulating the experimental CVs obtained. ${ }^{b}$ Values in parentheses are defined by other parameters simulated in the square scheme.

Monoamidinium salt 6, a cationic monomer of 4, provides insight into the effect of tethering on the ability of $\mathbf{4}$ to tightly bind to $\mathbf{Q}^{\bullet-}$. A comparison of the $\mathrm{K}_{3}$ values obtained using 4 and 6 reveals that this tethering of the cationic subunits improves binding by nearly seven orders of magnitude. This demonstrates that the intrinsic characteristics of the hydrogen-bonding moiety (e.g a phenyl-substituted amidinium salt vs. a phenyl-substituted guanidinium salt) are 
not solely responsible for the enhanced binding to $\mathbf{4}$ relative to HBDs $\mathbf{1}-\mathbf{3}$, and that the dicationic nature of the HBD is crucial. This has implications for further HBD development, as the linking of other monocationic HBDs could also afford improved binding.

\section{2a.5.8 Effect of conformational rigidity on binding ability}

Guanidinium salts such as $\mathbf{1}$ and $\mathbf{2}$ can access multiple binding conformations through bond rotation (Figure 2.25). In contrast, guanidinium salt 7 is a conformationally rigid structure with no rotational possibilities, which provides an opportunity to examine the effect of HBD flexibility on binding to $\mathbf{Q}^{\bullet-}$.
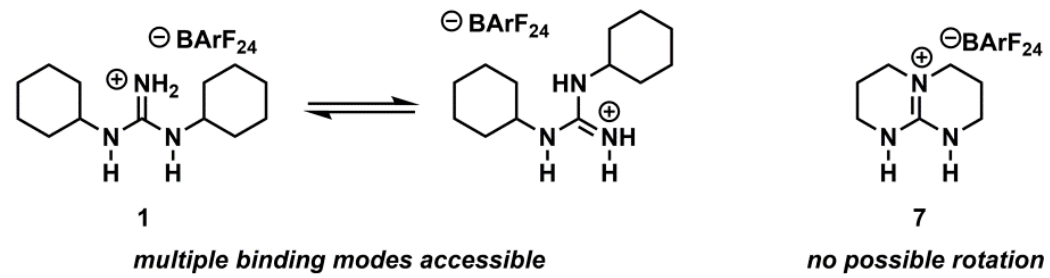

Figure 2.25. Conformational flexibility in guanidinium salt 1 vs. conformational rigidity in guanidinium salt 7.

Guanidinium salt 7 affords $\mathrm{CV}$ traces that are straightforwardly simulated by a CEC mechanism (Figure 2.26). The simulated curves neatly replicate the experimental curves, with the best overlap observed with 7 in comparison with the previous titration experiments. This may indicate that the equilibria between the multiple binding modes possible with $\mathbf{1}$ and $\mathbf{2}$ have some effect on the CVs recorded with these HBDs. 


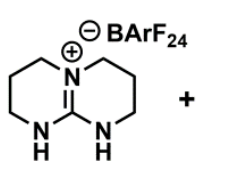

7

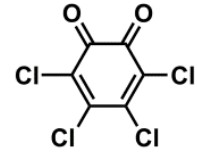

Q

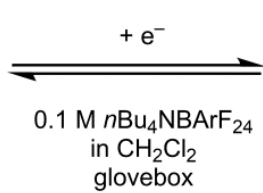

glovebox
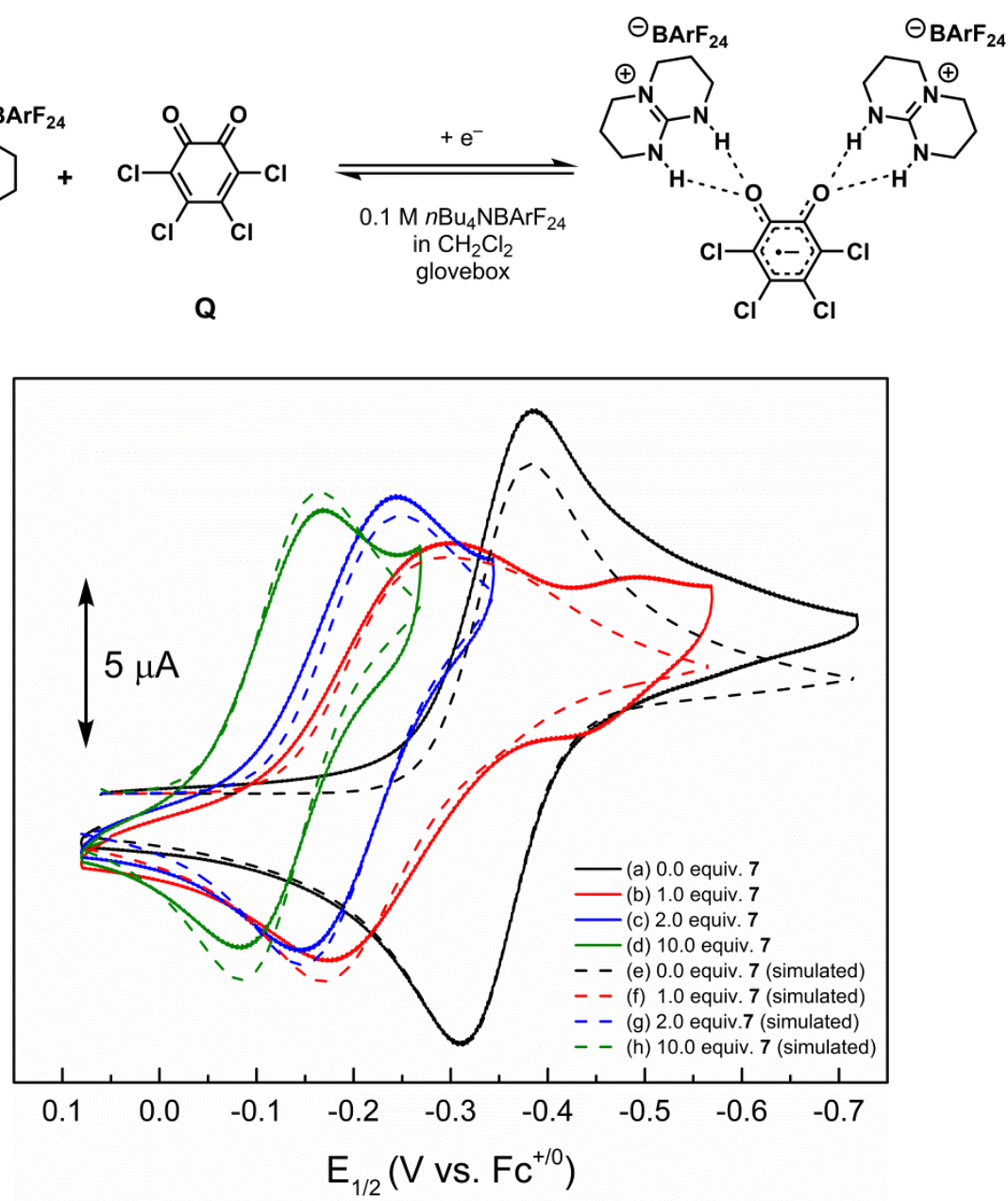

Figure 2.26. Experimental CVs of $o$-chloranil (initial concentration $=0.5 \mathrm{mM}$ ) recorded in 0.1 $\mathrm{M} n \mathrm{Bu}_{4} \mathrm{NBArF}_{24} / \mathrm{CH}_{2} \mathrm{Cl}_{2}$ (glovebox) in the presence of increasing [7] and comparison with simulation of a CEC mechanism. In scan (b), the additional current peaks at lower potential are attributed to the $2 \mathrm{e}^{-}$reduction.

The value for $\mathrm{K}_{3} \mathrm{~K}_{4}$ describing formation of $(7)_{2} \cdot \mathbf{Q}^{\cdot-}$ was calculated from the parameters used to simulate the data (Table 2.10), and was found to be $2.2 \times 10^{8} \mathrm{M}^{-2}$. This does not constitute a significant difference relative to $\mathbf{1}$, and indicates that the restricted conformation of the guanidinium salt does not substantially alter its binding ability. A slight decrease in binding ability is observed relative to $\mathbf{1}$ and could be attributed to differences in acidity between $\mathbf{1}$ and $\mathbf{7}$, as 7 is expected to be less acidic due to the alkylation on the H-bonding moiety. 
Table 2.10. Equations and best-fit parameters used to simulate $\mathrm{CV}$ data for $0.5 \mathrm{mM} o$-chloranil (Q) in $0.1 \mathrm{M} \mathrm{nBu} \mathrm{NBArF}_{24} / \mathrm{CH}_{2} \mathrm{Cl}_{2}$ (glovebox) in the presence of increasing [7]. Italicized values were fixed in the simulation.

\begin{tabular}{cccc}
\hline $\begin{array}{c}\text { Electron transfer } \\
\text { steps }\end{array}$ & $\mathbf{E}_{\mathbf{1 / 2}}\left(\mathbf{V}\right.$ vs. Fc $\left.\mathbf{c}^{\mathbf{0 / +}}\right)$ & $\boldsymbol{\alpha}$ & $\mathbf{k}_{\mathbf{s}}(\mathbf{c m} / \mathbf{s})$ \\
\hline $\mathbf{Q}+\mathrm{e}^{-} \rightarrow \mathbf{Q}^{\bullet-}$ & -0.35 & 0.5 & 0.02 \\
$\mathbf{7 \cdot} \mathbf{Q}+\mathrm{e}^{-} \rightarrow \mathbf{7} \cdot \mathbf{Q}^{\bullet-}$ & 0.11 & 0.5 & 1 \\
\hline $\mathbf{C h e m i c a l ~ s t e p s}$ & $\mathbf{K}_{\mathbf{e q}}\left(\mathbf{M}^{-\mathbf{1}}\right)$ & & $1 \times 10^{10}$ \\
\hline $\mathbf{7}+\mathbf{Q} \rightarrow \mathbf{7} \cdot \mathbf{Q}$ & 0.29 & $1 \times 10^{10}$
\end{tabular}

\section{2a.5.9 Mechanistic analysis of HBD-coupled electron transfer using formamidinium salts}

Formamidinium salt $\mathbf{8}$, which is analogous to $\mathbf{1}$, provides an opportunity to further interrogate the effects of acidity and charge distribution on hydrogen bonding to $\mathbf{Q}^{\bullet-}$. Consistent with all previous titrations using monocationic HBDs, the $\mathrm{CV}$ data obtained with $\mathbf{8}$ are best represented by a CEC mechanism (Figure 2.27). The apparent potential of the second-electron reduction is also substantially affected. The equations and parameters used to simulate the data collected with 6 are summarized in Table 2.11. 


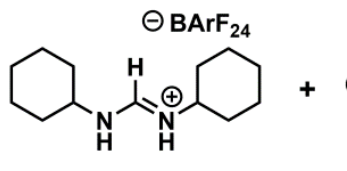

8

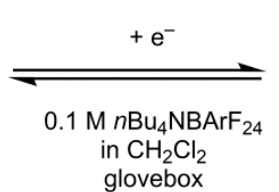

Q

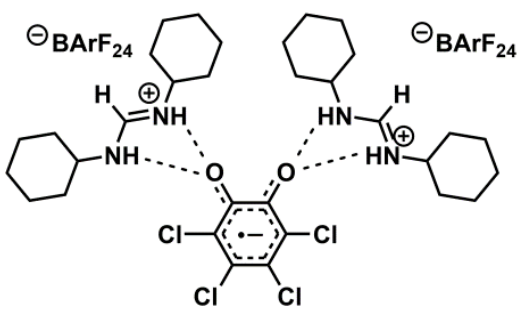

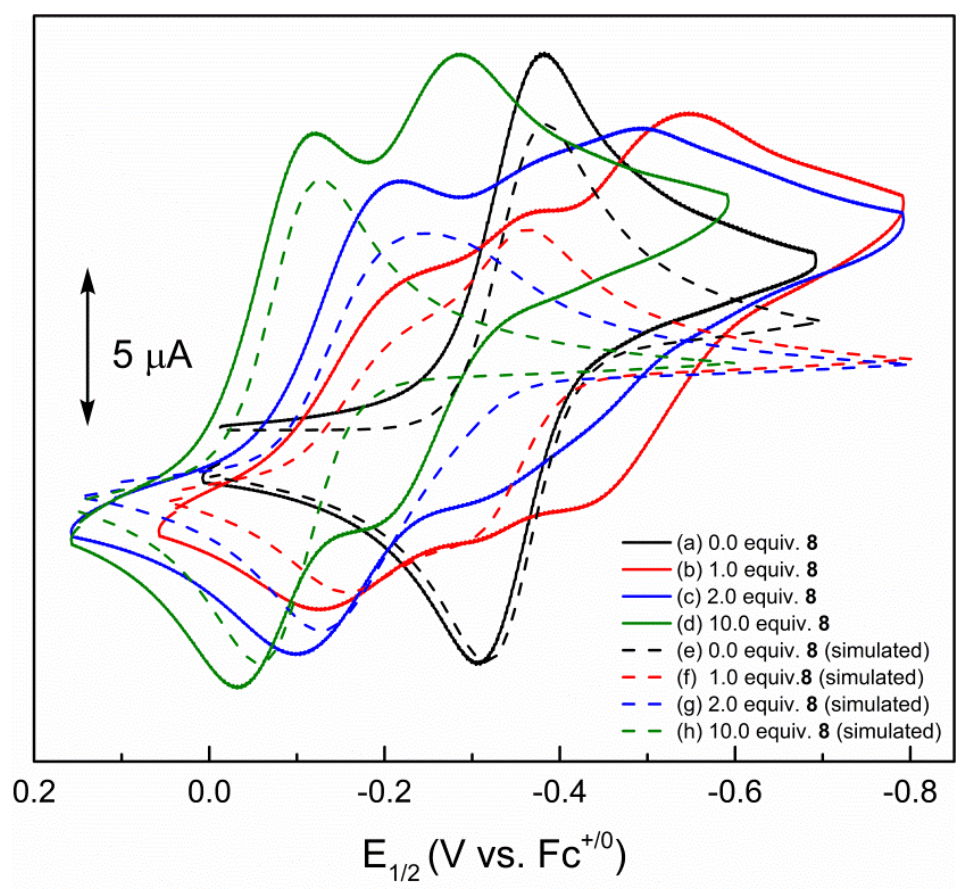

Figure 2.27. Experimental CVs of $o$-chloranil (initial concentration $=0.5 \mathrm{mM}$ ) recorded in 0.1 $\mathrm{M} n \mathrm{Bu}_{4} \mathrm{NBArF}_{24} / \mathrm{CH}_{2} \mathrm{Cl}_{2}$ (glovebox) in the presence of increasing [8] and comparison with simulation of a CEC mechanism. In scans (b)-(d), the additional current peaks at lower potential are attributed to the $2 \mathrm{e}^{-}$reduction.

Table 2.11. Equations and best-fit parameters used to simulate $\mathrm{CV}$ data for $0.5 \mathrm{mM}$-chloranil (Q) in $0.1 \mathrm{M} n \mathrm{Bu}_{4} \mathrm{NBArF}_{24} / \mathrm{CH}_{2} \mathrm{Cl}_{2}$ (glovebox) in the presence of increasing [7]. Italicized values were fixed in the simulation.

\begin{tabular}{cccc}
\hline $\begin{array}{c}\text { Electron transfer } \\
\text { steps }\end{array}$ & $\mathbf{E}_{\mathbf{1} / \mathbf{2}}\left(\mathbf{V} \mathbf{v s .} \mathbf{F c}^{\mathbf{0} /+}\right)$ & $\boldsymbol{\alpha}$ & $\mathbf{k}_{\mathbf{s}}(\mathbf{c m} / \mathbf{s})$ \\
\hline $\mathbf{Q}+\mathrm{e}^{-} \rightarrow \mathbf{Q}^{\bullet-}$ & -0.34 & 0.5 & 0.03 \\
$\mathbf{8} \cdot \mathbf{Q}+\mathrm{e}^{-} \rightarrow \mathbf{8} \cdot \mathbf{Q}^{--}$ & -0.19 & 0.5 & 1 \\
\hline $\mathbf{C h e m i c a l ~ s t e p s}$ & $\mathbf{K}_{\mathrm{eq}}\left(\mathbf{M}^{-\mathbf{1}}\right)$ & $\mathbf{k}_{\mathbf{f}}\left(\mathbf{s}^{-\mathbf{1}}\right)$ \\
\hline $\mathbf{8}+\mathbf{Q} \rightarrow \mathbf{8} \cdot \mathbf{Q}$ & 160 & $1 \times 10^{10}$ \\
$\mathbf{8} \cdot \mathbf{Q}^{\bullet-} \rightarrow(\mathbf{8})_{\mathbf{2}} \cdot \mathbf{Q}^{\bullet-}$ & $3.5 \times 10^{4}$ & $1 \times 10^{10}$
\end{tabular}


A comparison of the values for $\mathrm{K}_{3} \mathrm{~K}_{4}$ obtained with HBDs 1, 2, and $\mathbf{8}$ shows that the formamidinium salt offers intermediate stability relative to $\mathbf{1}$ and $\mathbf{2}$ (Table 2.12). The improved binding of $\mathbf{8}$ relative to $\mathbf{1}$ can be attributed to increased acidity in the formamidinium salt, which offers decreased stability through resonance compared with the guanidinium salt. These differences further emphasize the importance of hydrogen bonding in this system.

Table 2.12. Summary of parameters for HBD-coupled electron transfer promoted by HBDs 1-3.

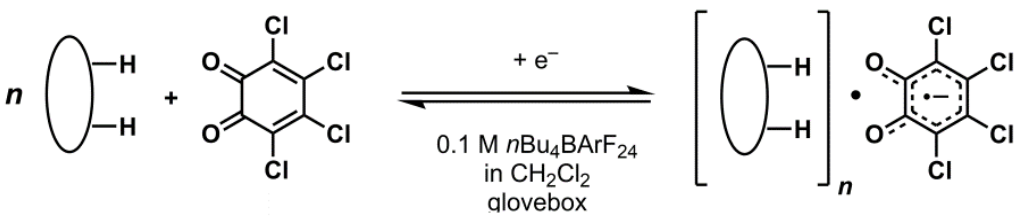

\begin{tabular}{ccccc}
\hline HBD & $\mathrm{K}_{1}\left(\mathrm{M}^{-1}\right)$ & $\mathrm{E}_{2}(\mathrm{~V})$ & $\mathrm{K}_{3}\left(\mathrm{M}^{-1}\right)^{\mathrm{b}}$ & $\mathrm{K}_{3} \mathrm{~K}_{4}\left(\mathrm{M}^{-2}\right)$ \\
\hline $\mathbf{1}$ & 66 & -0.17 & $\left(3.4 \times 10^{4}\right)$ & $6.1 \times 10^{8}$ \\
$\mathbf{2}$ & 5.5 & -0.057 & $\left(3.5 \times 10^{5}\right)$ & $1.8 \times 10^{10}$ \\
$\mathbf{8}$ & 157 & -0.19 & $\left(3.5 \times 10^{4}\right)$ & $2.1 \times 10^{9}$
\end{tabular}

${ }^{a}$ Parameters were determined by titrating $0.5 \mathrm{mM} \mathrm{Q}$ in $0.1 \mathrm{M} n \mathrm{Bu}_{4} \mathrm{NBArF}_{24} / \mathrm{CH}_{2} \mathrm{Cl}_{2}$ (glovebox) with [HBD] and simulating the experimental CVs obtained. ${ }^{b}$ Values in parentheses are defined by other parameters simulated in the square scheme.

This same resonance effect also impacts charge distribution, which in turn could affect the electrostatic attraction between the charged HBDs and $\mathbf{Q}^{\bullet-}$ (Figure 2.28). Charge delocalization in $\mathbf{1}$ in greater than that in $\mathbf{8}$, in which the positive character is concentrated at the H-bonding moiety. In comparison, the charge delocalization in $\mathbf{1}$ results in decreased cationic character at the H-bonding site. Based on the pronounced effect of electrostatic interactions, which has been discussed above, we hypothesize that this charge localization may contribute to 
the enhanced stabilization provided by $\mathbf{8}$ to $\mathbf{Q}^{\bullet-}$. Because acidity and electrostatics are so closely intertwined, it is difficult to determine precisely how resonance and charge distribution independently affect the ability of an HBD to bind to $\mathbf{Q}^{\bullet-}$. Charge distribution, however, could be an important consideration in design of future HBDs that promote electron transfer.
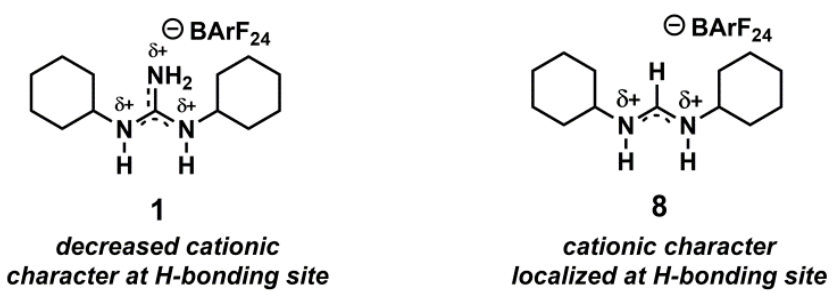

Figure 2.28. Differences in charge distribution between 1 and $\mathbf{8}$.

Based on the observed effect of increased acidity on improved H-bonding ability, we expected that diphenylformamidinium salt $\mathbf{9}$ would exhibit improved binding to $\mathbf{Q}^{--}$, relative to $\mathbf{2}$ and 8. The CV experiments afford complex traces that prove difficult to analyze through simulations (Figure 2.29). These data present similar features as observed with 4 , with extra current appearing between the peaks that correspond to the $\mathbf{Q} / \mathbf{Q}^{\bullet-}$ wave and the $\mathbf{Q}^{\bullet-} / \mathbf{Q}^{2-}$ wave. This extra current seems to be related to acidity, as it only appears in the CV traces recorded with the most acidic HBDs. The literature explanations for this current are likely applicable for $\mathbf{9}$ as well (vide supra). In addition to these complicating features, irreversibility in the CV traces is also observed as the titration progresses. 


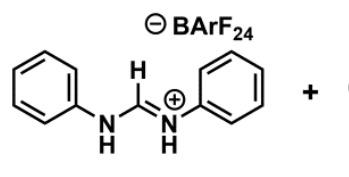

9<smiles>O=C1C(=O)C(Cl)=C(Cl)C(Cl)=C1Cl</smiles>

Q

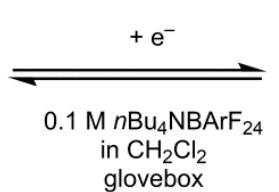

glovebox
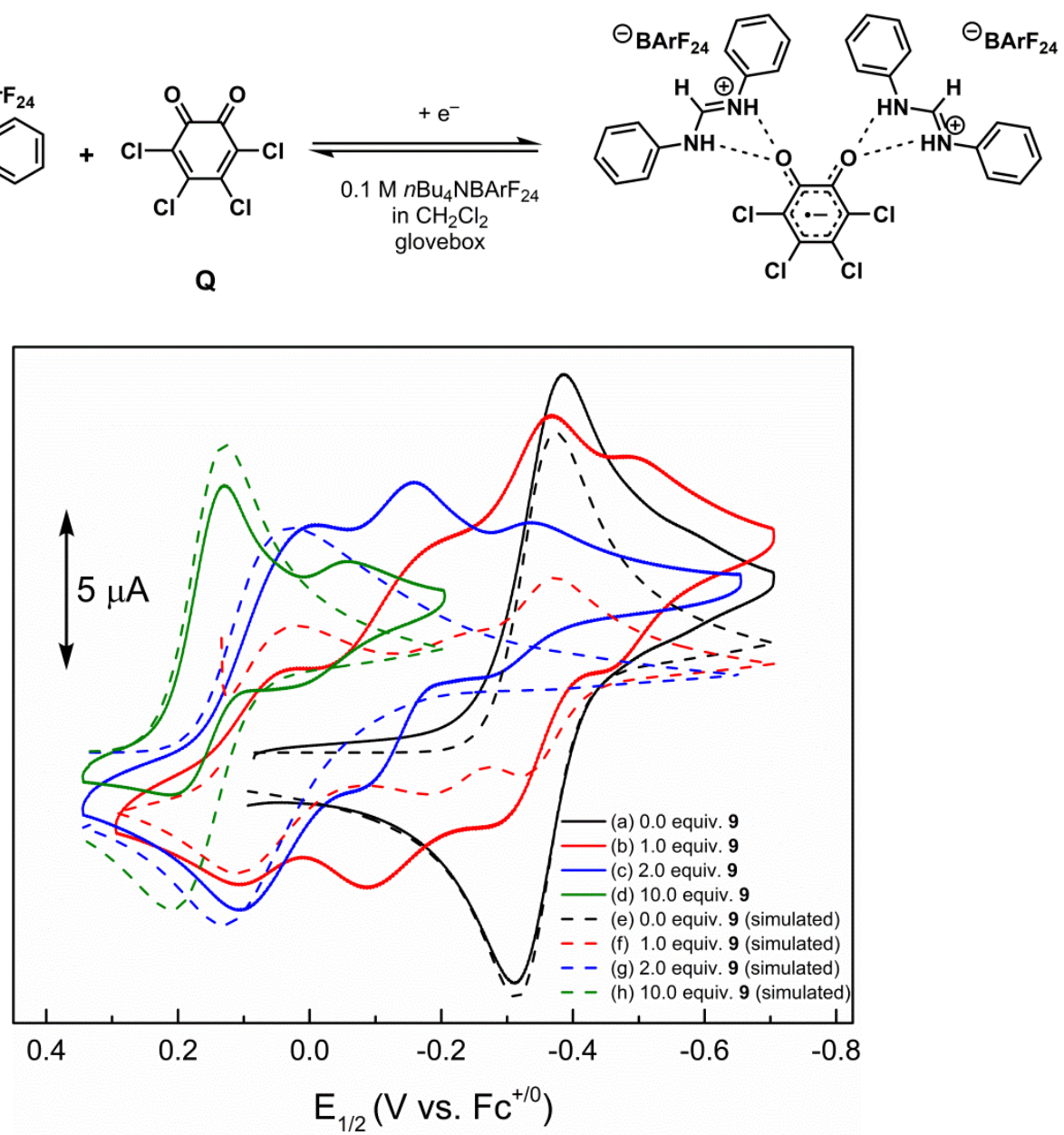

Figure 2.29. Experimental CVs of $o$-chloranil (initial concentration $=0.5 \mathrm{mM}$ ) recorded in 0.1 $\mathrm{M} n \mathrm{Bu}_{4} \mathrm{NBArF}_{24} / \mathrm{CH}_{2} \mathrm{Cl}_{2}$ (glovebox) in the presence of increasing [9] and comparison with simulation of a CEC mechanism. In scans (b)-(d), the additional current peaks at lower potential are attributed to the $2 \mathrm{e}^{-}$reduction.

Simulations of a CEC mechanism are unable to explain the extra current, but do reproduce both the overall $\Delta \mathrm{E}_{1 / 2}$ as well as the developing irreversibility (Figure 2.30). This indicates that 9 behaves consistently with the monocationic HBDs and forms a 2:1 complex with $\mathbf{Q}^{\bullet-}$. In this case, the irreversibility could be a result of very tight binding in the $(\mathbf{9})_{2} \cdot \mathbf{Q}^{\bullet-}$ complex, which depletes $\left[\mathbf{Q}^{\bullet-}\right]$ at the electrode surface and results in diminished current corresponding to its reoxidation. The observed irreversibility could also be consistent with proton transfer between 
$\mathbf{Q}^{\bullet-}$ and $\mathbf{9}$ to give $\mathbf{Q} \mathbf{H}^{\bullet}$ (Figure 2.31), although inclusion of an explicit protonation event within the simulations degrades the quality of the fit.

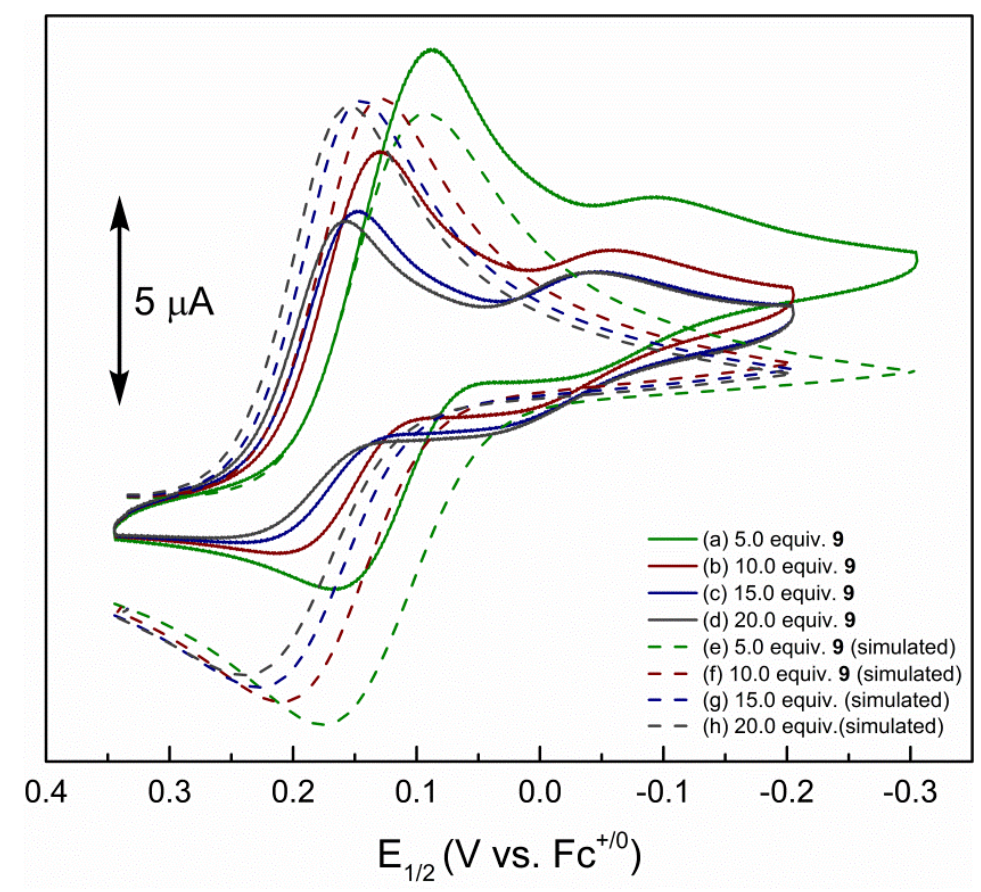

Figure 2.30. Experimental CVs of $o$-chloranil (initial concentration $=0.5 \mathrm{mM}$ ) recorded in 0.1 $\mathrm{M} n \mathrm{Bu}_{4} \mathrm{NBArF}_{24} / \mathrm{CH}_{2} \mathrm{Cl}_{2}$ (glovebox) in the presence of increasing [9] and comparison with simulation of a CEC mechanism. Only data at large [9] are shown. In scans (a)-(d), the additional current peaks at lower potential are attributed to the $2 \mathrm{e}^{-}$reduction.
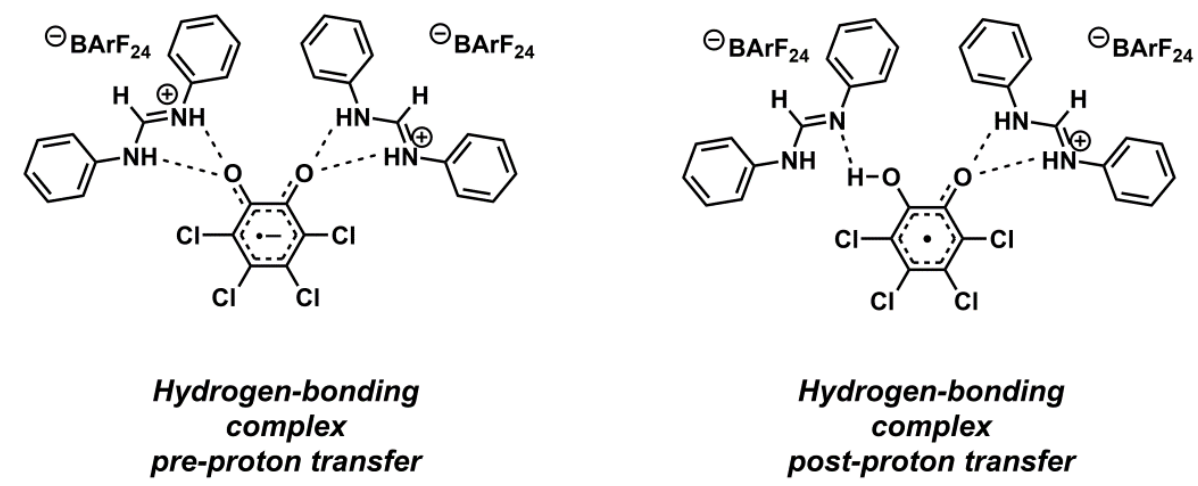

Figure 2.31. 2:1 complexes between 9 and the reduced quinone involving H-bonding and protonation. 
The parameters that describe the CEC simulation reveal that strong binding may in fact account for the observed irreversibility, as the value obtained for $\mathrm{K}_{3} \mathrm{~K}_{4}$ is $4.3 \times 10^{13} \mathrm{M}^{-2}$ and indicates that 9 is the most potent of the monocationic HBDs studied here (Table 2.13). When the $K_{3}$ values for 4 and 9 are compared, the diphenylformamidinium salt is still weaker than the bis-amidinium salt by three orders of magnitude. Further study is needed to establish the stability of the $(\mathbf{9})_{2} \cdot \mathbf{Q}^{\bullet-}$ complex and whether $\mathbf{9}$ would be viable as a catalyst for HBD-coupled electron transfer. Although the origin of the large effect observed with 9 is uncertain, we present these results here principally because they indicate promise for the use of HBDs similar to 9, and could inform future HBD design.

Table 2.13. Equations and best-fit parameters used to simulate $\mathrm{CV}$ data for $0.5 \mathrm{mM} o$-chloranil (Q) in $0.1 \mathrm{M} n \mathrm{Bu}_{4} \mathrm{NBArF}_{24} / \mathrm{CH}_{2} \mathrm{Cl}_{2}$ (glovebox) in the presence of increasing [9]. Italicized values were fixed in the simulation.

\begin{tabular}{cccc}
\hline $\begin{array}{c}\text { Electron transfer } \\
\text { steps }\end{array}$ & $\mathbf{E}_{\mathbf{1} / \mathbf{2}}\left(\mathbf{V}\right.$ vs. Fc $\left.\mathbf{c}^{\mathbf{0 / +}}\right)$ & $\boldsymbol{\alpha}$ & $\mathbf{k}_{\mathbf{s}}(\mathbf{c m} / \mathbf{s})$ \\
\hline $\mathbf{Q}+\mathrm{e}^{-} \rightarrow \mathbf{Q}^{\bullet-}$ & -0.34 & 0.5 & 0.1 \\
$\mathbf{9} \cdot \mathbf{Q}+\mathrm{e}^{-} \rightarrow \mathbf{9} \cdot \mathbf{Q}^{\bullet-}$ & -0.01 & 0.5 & 10 \\
\hline $\mathbf{C h e m i c a l ~ s t e p s}$ & $\mathbf{K}_{\mathbf{e q}}\left(\mathbf{M}^{-\mathbf{1}}\right)$ & & $1 \times 10^{10}$ \\
\hline $\mathbf{9}+\mathbf{Q} \rightarrow \mathbf{9} \cdot \mathbf{Q}$ & 120 & $1 \times 10^{10}$
\end{tabular}

\section{2a.6 Conclusions from electrochemical experiments}

The values obtained for $\mathrm{K}_{3}$ and $\mathrm{K}_{3} \mathrm{~K}_{4}$ are summarized in Table 2.14 for the HBDs that could be relevant in terms of future catalyst design. Comparison of these values allows us to establish that acidity and electrostatics are thoroughly intertwined in promoting HBD-coupled electron transfer. 
Table 2.14. Summary of parameters for HBD-coupled electron transfer promoted by HBDs 1-3.

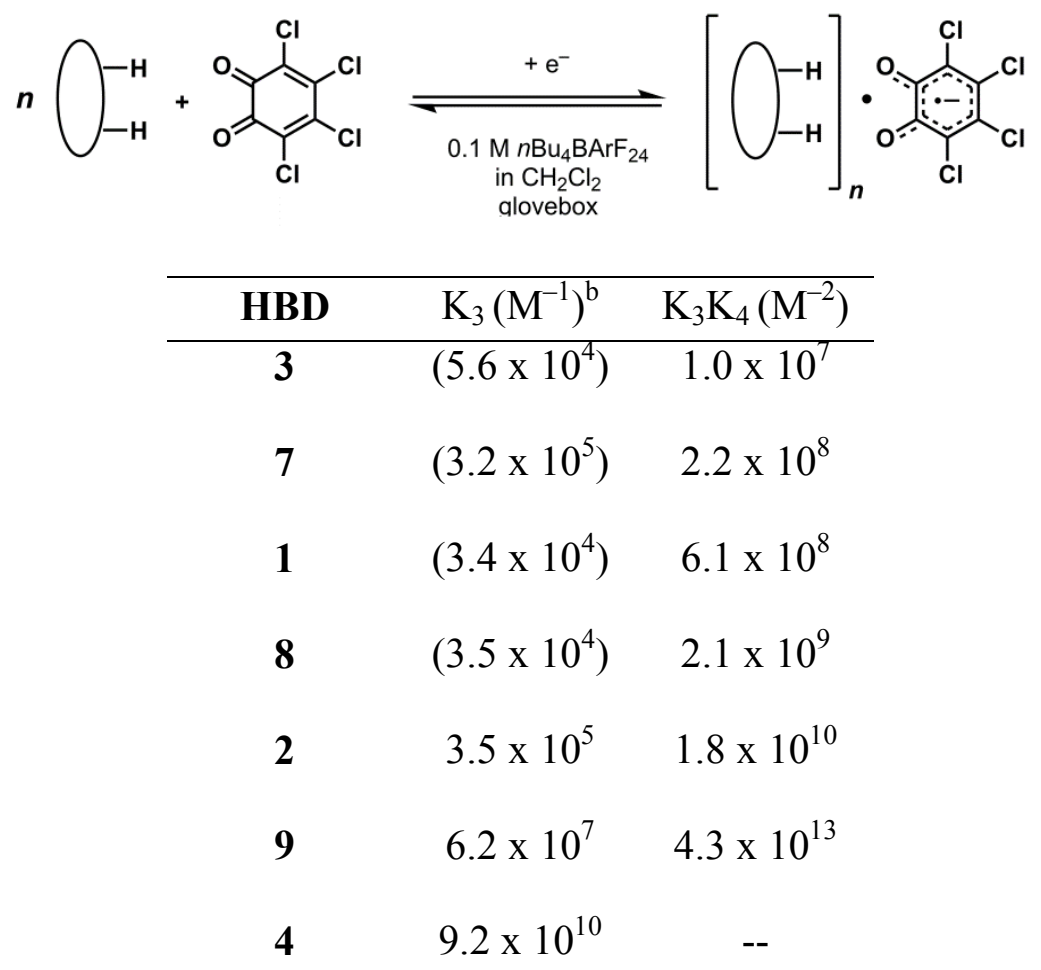

While the general increase in binding ability from neutral $\mathbf{3}$ and the superior binding ability of dicationic 4 illustrates a crucial role for electrostatics, other trends indicate that acidity is also important (e.g $\mathbf{1}<\mathbf{2}, \mathbf{1}<\mathbf{8}$ ). The observation that two cationic HBDs act in tandem to achieve electron transfer culminated in the discovery that a linked, dicatonic HBD provides the most significant degree of stabilization to the reduced quinone, thereby favorably modulating the thermodynamics of electron transfer. These results, taken together, will inform design of future HBDs that bind potently to $\mathbf{Q}^{\bullet-}$. 


\section{2a.7 Experimental details}

\section{2a.7.1 General information}

Commercial reagents were purchased from Sigma-Aldrich, Alfa Aesar, Acros, Strem Chemicals, and Matrix Scientific, and were used as received unless otherwise noted. Dichloromethane, $N, N$-dimethylformamide, diethyl ether, tert-butyl methyl ether, and tetrahydrofuran were purified by passing through a column of activated alumina. Triethylamine was freshly distilled over $\mathrm{CaH}_{2}$ at 760 torr prior to use. Cyclohexylamine was obtained by treating the crystalline hydrochloride salt with base. Sodium $\mathrm{BArF}_{24}{ }^{14}$ and $\mathrm{HBArF}_{24} \cdot \mathrm{Et}_{2} \mathrm{O}^{15}$ were prepared according to procedures found in the literature. Sodium $\mathrm{BArF}_{24}, \mathrm{TBABArF}_{24}$, and all hydrogen bond donors were stored in a vacuum desiccator over Drierite ${ }^{\circledR}$ and $\mathrm{P}_{2} \mathrm{O}_{5}$. $\mathrm{HBArF}_{24} \cdot \mathrm{Et}_{2} \mathrm{O}$ was stored as a solid at $-80{ }^{\circ} \mathrm{C}$. Column chromatography was performed on a Biotage Isolera automated purification system using EM Science silica gel 60 (230-400 mesh) and the indicated eluent. Analytical TLC was performed using EM Separations pre-coated silica gel $0.2 \mathrm{~mm}$ layer UV 254 fluorescent sheets. All glass reaction vessels were oven-dried, evacuated and backfilled with nitrogen three times prior to use unless otherwise noted. Solvents and solutions were transferred by syringes or cannulae using standard inert atmosphere techniques.

Proton nuclear magnetic resonance $\left({ }^{1} \mathrm{H}\right.$ NMR) and carbon nuclear magnetic resonance $\left({ }^{13} \mathrm{C}\right.$ NMR) spectra were obtained using an Inova-500 (500 MHz) or Mercury $400(400 \mathrm{MHz})$ spectrometer. ${ }^{1} \mathrm{H}$ NMR spectra were referenced to residual solvent peaks (using the indicated solvent). ${ }^{13} \mathrm{C}$ NMR spectra were referenced to solvent carbons. Infrared (IR) spectra were obtained using a Bruker Optics Tensor 27 FTIR spectrometer. Spectra were obtained using thin

\footnotetext{
${ }^{14}$ Yakelis, N. A.; Bergman, R. G. Organometallics 2005, 24, 3579.

${ }^{15}$ Brookhart, M.; Grant, B.; Volpe, Jr., A. F. Organometallics 1992, 11, 3920
} 
films of products. High resolution mass spectroscopic (HRMS) data were obtained using a Bruker microTOF-Q II time-of-flight LC/MS spectrometer. Low resolution mass spectroscopic data were obtained using an Agilent 6120 Single Quadrupole LC/MS instrument equipped with an ESI/APCI multimode source.

\section{2a.7.2 Procedures for the syntheses of HBDs 1-9}

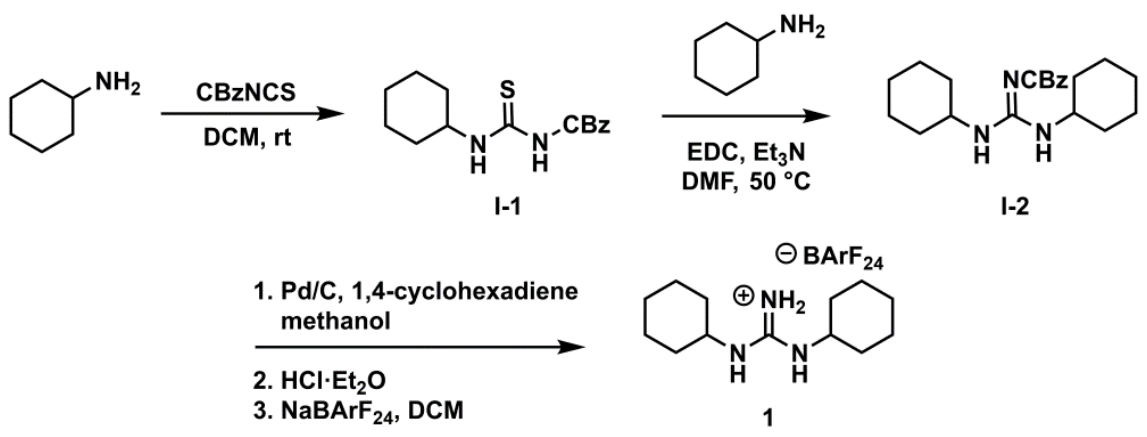

\section{$N$-Benzyloxycarbonyl- $N$ '-cyclohexylthiourea (I-1)}

CBzNCS ${ }^{16}$ (4.0 g, 20.7 mmol, 1 equiv.) was dissolved in DCM $(70 \mathrm{~mL})$. Cyclohexylamine (2.4 mL, $20.7 \mathrm{mmol}, 1$ equiv.) was added with stirring at $\mathrm{rt}$, and the reaction mixture was stirred for 6 hours. The solution was then concentrated via rotary evaporation in vacuo, and was loaded directly onto silica gel. Column chromatography $(0-5 \% \mathrm{MeOH}$ in $\mathrm{DCM})$ afforded the product as a white solid $(6.4 \mathrm{~g},>99 \%$ yield $)$.

${ }^{1}$ H NMR (500 MHz, $\mathrm{CDCl}_{3}$ ) $\delta 9.58$ (br. s, 1H), 8.01 (br. s, 1H), 7.41-7.34 (br. m, 5H), 5.17 (s, 2H), 4.27-4.21 (br. m, 2H), 2.06-2.03 (br. ap. d, 2H), 1.72-1.70 (br. ap. d., 2H), 1.64-1.60 (m, $1 \mathrm{H}), 1.46-1.22(\mathrm{~m}, 5 \mathrm{H})$;

\footnotetext{
${ }^{16}$ Lanman, B. A.; Overman, L. E.; Paulini, R.; White, N. S. J. Am. Chem. Soc. 2007, 129, 12896.
} 
${ }^{13} \mathbf{C}\left\{{ }^{1} \mathbf{H}\right\}$ NMR (125 MHz, $\left.\mathrm{CDCl}_{3}\right) \delta 177.3,152.4,134.5,128.9,128.8,128.3,68.1,54.2,31.7$, 25.4, 24.4;

IR (film) $v_{\max } 3288,3245,3177,2931,2854,1715,1536,1452,1402,1346,1225,1197,1180$, $1025,772,738,696 \mathrm{~cm}^{-1}$;

MS (ESI-TOF) calcd. for $\mathrm{C}_{15} \mathrm{H}_{20} \mathrm{~N}_{2} \mathrm{O}_{2} \mathrm{~S}\left[\mathrm{M}+\mathrm{H}^{+}\right]$293.1324, found 293.1363 .

\section{$N$-benzyloxycarbonyl- $N$ ', $N$ ',-dicyclohexylguanidine (I-2)}

Thiourea I-1 (0.75 g, $2.6 \mathrm{mmol}, 1.0$ equiv) was dissolved in DMF (30 mL) and cyclohexylamine ( $0.3 \mathrm{~mL}, 2.6 \mathrm{mmol}, 1.0$ equiv) was added. To the stirred solution was added EDC (1.25 g, $6.5 \mathrm{mmol}, 2.5$ equiv.) followed by triethylamine ( $1.8 \mathrm{~mL}, 13 \mathrm{mmol}, 5.0$ equiv), and the reaction mixture was heated to $50{ }^{\circ} \mathrm{C}$ for $12 \mathrm{~h}$. The reaction mixture was cooled to $\mathrm{rt}$, was transferred to a separatory funnel, and was extracted with EtOAc. The organic phase was washed with water $(2 \times 100 \mathrm{~mL}), 1 \mathrm{~N} \mathrm{HCl}(2 \times 100 \mathrm{~mL})$, saturated aqueous $\mathrm{NaHCO}_{3}(2 \times 100 \mathrm{~mL})$, and brine $(1 \times 100 \mathrm{~mL})$. The organic phase was dried over $\mathrm{Na}_{2} \mathrm{SO}_{4}$ and was concentrated via rotary evaporation in vacuo. Column chromatography on silica gel (0-10\% $\mathrm{MeOH}$ in DCM) yielded the product as a colorless oil $(0.73 \mathrm{~g}, 79 \%$ yield $)$.

${ }^{1}$ H NMR $\left(500 \mathrm{MHz}, \mathrm{CDCl}_{3}\right) \delta 7.42-7.40(\mathrm{~d}, 2 \mathrm{H}, \mathrm{J}=7.2 \mathrm{~Hz}), 7.34-7.31(\mathrm{t}, 2 \mathrm{H}, \mathrm{J}=7.0 \mathrm{~Hz}), 7.26-$ $7.24(\mathrm{~m}, 1 \mathrm{H}), 5.12(\mathrm{~s}, 2 \mathrm{H}), 1.95-1.93(\mathrm{~m}, 4 \mathrm{H}), 1.73-1.70(\mathrm{~m}, 4 \mathrm{H}), 1.61-1.58(\mathrm{~m}, 2 \mathrm{H}), 1.41-1.33$ (m, 4H), 1.27-1.22 (br. m, 6H);

${ }^{13} \mathbf{C}\left\{{ }^{1} \mathbf{H}\right\}$ NMR $\left(125 \mathrm{MHz}, \mathrm{CDCl}_{3}\right) \delta 164.2,158.4,137.8,128.2,127.9,127.5,66.3,49.4,33.2$, 25.5, 24.5;

IR (film) $v_{\max } 2928,2853,1592,1450,1397,1308,1297,1273,1133,1085,1071,1028,997$, $798,731,697 \mathrm{~cm}^{-1}$; 
MS (ESI-TOF) calcd. for $\mathrm{C}_{21} \mathrm{H}_{31} \mathrm{~N}_{3} \mathrm{O}_{2}\left[\mathrm{M}+\mathrm{H}^{+}\right] 358.2495$, found 358.2573 .

\section{$N, N$ '-dicyclohexylguanidinium tetrakis(3,5-bis(trifluoromethyl)phenyl)borate (1)}

A round-bottomed flask was charged with $\mathrm{Pd} / \mathrm{C}(1.3 \mathrm{~g}, 200 \mathrm{wt} \%$ with respect to I-2), which was slurried with EtOAc. Methanol $(27 \mathrm{~mL})$ was slowly added to the slurry. I-2 $(0.67 \mathrm{~g}$, $1.87 \mathrm{mmol}, 1.0$ equiv.) was transferred to the flask using $\mathrm{MeOH}(10 \mathrm{~mL})$. The flask was submerged in a rt water bath and 1,4-cyclohexadiene (3.54 mL, $37.4 \mathrm{mmol}, 20$ equiv.) was added. The reaction was stirred and monitored by mass spectrometry until no starting material remained $(\sim 12-24 \mathrm{~h})$. The reaction mixture was filtered through Celite and rinsed with $\mathrm{MeOH}(\sim 25 \mathrm{~mL})$. The filtrate was cooled to $0{ }^{\circ} \mathrm{C}$ and $\mathrm{HCl}\left(1.87 \mathrm{~mL}, 2.0\right.$ equiv, $2 \mathrm{M}$ in $\left.\mathrm{Et}_{2} \mathrm{O}\right)$ was added dropwise. The reaction mixture was stirred for 10 minutes and was concentrated via rotary evaporation in vacuo. The residue was suspended in $\mathrm{DCM}$ and $\mathrm{NaBArF}_{24}(1.66 \mathrm{~g}, 1.87 \mathrm{mmol}, 1.0$ equiv.) was added. The suspension was stirred for 15 minutes and a fine white precipitate formed, and the reaction mixture was then filtered through Celite. The filtrate was concentrated via rotary evaporation in vacuo to afford a colorless oil. Column chromatography on silica gel $(0-5 \%$ $\mathrm{MeOH}$ in DCM) yielded the product as a white solid (0.52 g, 26\% yield).

${ }^{1} \mathbf{H}$ NMR $\left(500 \mathrm{MHz}, \mathrm{CDCl}_{3}\right) \delta 7.69(\mathrm{~s}, 8 \mathrm{H}), 7.55(\mathrm{~s}, 4 \mathrm{H}) ;$ 5.03-4.97 (br.m, 4H), 3.16-3.10 (m, 2H), 1.88-1.84 (m, $4 \mathrm{H}), 1.75-1.65$ (m, 6H), 1.34-1.14 (m, 10H);

${ }^{13} \mathbf{C} \mathbf{N M R}\left\{{ }^{1} \mathbf{H}\right\}\left(125 \mathrm{MHz}, \mathrm{CDCl}_{3}\right) \delta 161.6(\mathrm{q}, \mathrm{J}=49.8 \mathrm{~Hz}), 152.6,134.7,128.9(\mathrm{q}, \mathrm{J}=28.7 \mathrm{~Hz})$, 124.5 (q, J=272.4 Hz), 117.6, 52.3, 32.5, 24.4, 24.0;

IR (film) $v_{\max } 3546,3434,2943,2865,1632,1611,1354,1276,1121,887,838,711,681,670$ $\mathrm{cm}^{-1}$;

MS (ESI-TOF) calcd. for $\mathrm{C}_{21} \mathrm{H}_{31} \mathrm{~N}_{3} \mathrm{O}_{2}$ [M - BAr $\left.{ }_{24}^{\mathrm{F}}\right]$ 224.2127, found 224.2204. 


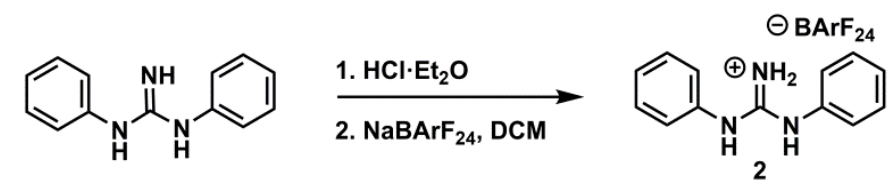

\section{$N, N$ '-diphenylguanidinium tetrakis(3,5-bis(trifluoromethyl)phenyl)borate (2)}

Diphenylguanidinium $\mathrm{BArF}_{24} 2$ is a known compound and was synthesized from commercially available $N, N^{\prime}$-diphenylguanidine as reported in the literature. ${ }^{17}$ Spectral data matched that which has previously been reported.
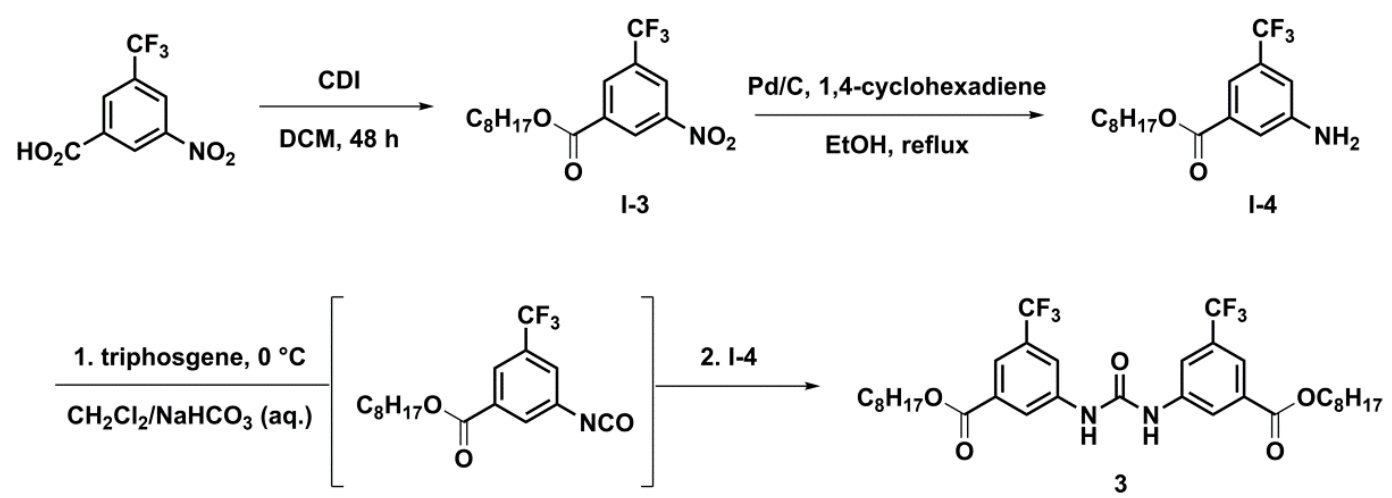

\section{Octyl 3-nitro-5-(trifluoromethyl)benzoate (I-3)}

Octyl 3-nitro-5-(trifluoromethyl)benzoate (I-3) is a known compound and was synthesized from commercially available 3-nitro-5-(trifluoromethyl)benzoic acid as reported in the literature. ${ }^{18}$ Excess 1 -octanol was removed by distillation at reduced pressure $(\sim 1$ torr $)$ prior to purification by chromatography. Spectral data matched that which was previously reported.

\footnotetext{
${ }^{17}$ Uyeda, C.; Jacobsen, E. N. J. Am. Chem. Soc. 2008, 138, 9228-9229.

${ }^{18}$ Curran, D. P.; Kuo, L. H. J. Org. Chem. 1994, 59, 3259-3261.
} 


\section{Octyl 3-amino-5-(trifluoromethyl)benzoate (I-4)}

Octyl 3-amino-5-(trifluoromethyl)benzoate (I-4) is a known compound and was synthesized from I-3 by a slight modification of the previously reported procedure. ${ }^{18}$ To a solution of I-3 (1.91 g, $5.5 \mathrm{mmol}, 1.0$ equiv) and 1,4-cyclohexadiene (2.6 mL, $27.5 \mathrm{mmol}, 5.0$ equiv) in EtOH $(11 \mathrm{~mL}, 0.5 \mathrm{M})$ was added palladium on carbon (50 wt. \% with respect to $\mathbf{I}-3)$. The reaction mixture was heated to reflux until no starting material remained by TLC analysis (3 h). After cooling to rt, the reaction mixture was filtered through Celite and washed with $\mathrm{MeOH}$ $(\sim 50 \mathrm{~mL})$, and the filtrate was concentrated via rotary evaporation in vacuo. Column chromatography on silica gel (0-25\% EtOAc in hexanes) afforded the product as a white solid (0.99 g, 57\% yield). Spectral data matched that which was previously reported.

\section{$N, N$ 'bis-(3-trifluoromethyl-5-carbooctyloxyphenyl) urea (3)}

$N, N$ '-bis-(3-trifluoromethyl-5-carbooctyloxyphenyl) urea (3) is a known compound ${ }^{18}$ and was prepared by a different procedure as follows. Triphosgene $(0.23 \mathrm{~g}, 0.8 \mathrm{mmol}, 0.5 \mathrm{guiv}$.) (caution: highly toxic) was dissolved in DCM. In a separate flask, I-4 (0.5 g, $1.6 \mathrm{mmol}, 1.0$ equiv.) was dissolved in a 1:1 mixture of sat. aq. sodium bicarbonate and DCM (0.15 M) and the solution was cooled to $0{ }^{\circ} \mathrm{C}$. Stirring was stopped and the triphosgene solution was added directly to the organic phase of the biphasic reaction mixture. The reaction was stirred at $0{ }^{\circ} \mathrm{C}$ for 30 minutes, and was warmed to $\mathrm{rt}$ and was stirred for $16 \mathrm{~h}$. The reaction mixture was transferred to a separatory funnel and was extracted with DCM $(3 \times 20 \mathrm{~mL})$. The organic phase was washed with brine and dried over sodium sulfate, and was filtered and concentrated via rotary evaporation in vacuo to afford the isocyanate as a colorless oil, which was carried forward without further purification. The crude isocyanate was dissolved in DCM (4 mL) and I-4 (0.51 g, 
$1.6 \mathrm{mmol}, 1.0$ equiv) was added as a solution in DCM $(4 \mathrm{~mL})$, and the reaction mixture was stirred for $36 \mathrm{~h}$. The reaction mixture was concentrated in vacuo to afford a white solid. Trituration with DCM afforded the product urea as a white solid ( $0.6 \mathrm{~g}, 57 \%$ yield). Spectral data matched that which was previously reported.

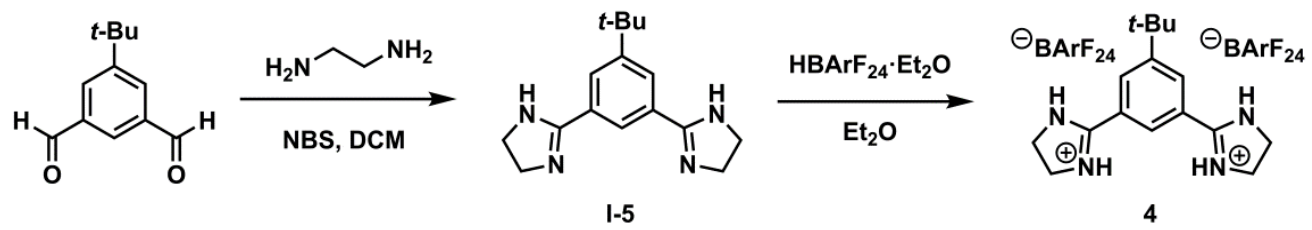

\section{2,2'-(5-(Tert-butyl)-1,3-phenylene)bis(4,5-dihydro-1H-imidazole) (I-5)}

5-(Tert-butyl)isophthalaldehyde was prepared in two steps from commercially available 5-(tert-butyl)isophthalic acid as reported in the literature. ${ }^{19}$ 2,2'-(5-(Tert-butyl)-1,3phenylene)bis(4,5-dihydro-1H-imidazole) (I-5) is a known compound ${ }^{20}$ that was prepared by a different procedure as follows. 5-(Tert-butyl)isophthalaldehyde (0.5 g, $2.6 \mathrm{mmol}, 1.0$ equiv) was dissolved in DCM (26 mL). Ethylenediamine (0.35 mL, $5.2 \mathrm{mmol}, 2.0$ equiv) was added and the reaction mixture was stirred for $20 \mathrm{~min}$. The reaction mixture was cooled to $0{ }^{\circ} \mathrm{C}$ and $\mathrm{N}$ bromosuccinimide (0.93 g, $5.2 \mathrm{mmol}, 2.0$ equiv) was added portionwise. The reaction mixture was stirred for $12 \mathrm{~h}$ at $\mathrm{rt}$, after which it was slowly quenched with sat. aq. sodium bicarbonate. The solution was transferred to a separatory funnel, and the aqueous phase was washed with DCM and the organic phase was discarded. The aqueous layer was basified with $1 \mathrm{M}$ sodium hydroxide until a white precipitate formed $(\mathrm{pH} \sim 12)$. The aqueous layer was extracted with EtOAc $(3 \times 100 \mathrm{~mL})$ and the combined organic layers were washed with brine, dried over $\mathrm{Na}_{2} \mathrm{SO}_{4}$,

\footnotetext{
${ }^{19}$ Bennani, Y. L.; Marron, K. S.; Mais, D. E.; Flatten, K.; Nadzan, A. M.; Boehm, M. F. J. Org. Chem. 1998, 63, 543.

${ }^{20}$ Kozlowski, M.C.; Annamalai, V.R.; Linton, E.C. Org. Lett. 2009, 11, 621-624.
} 
and concentrated in vacuo to yield the product as a white solid ( $0.56 \mathrm{~g}, 80 \%$ yield). Spectral data was identical to that which was previously reported.

\section{2,2'-(5-(Tert-butyl)-1,3-phenylene)bis(4,5-dihydro-1H-imidazol-3-ium) tetrakis(3,5- bis(trifluoromethyl)phenyl)borate (4)}

Bis-amidinium salt 4 is a known compound ${ }^{20}$ that was prepared by a different procedure as follows. A $50 \mathrm{~mL}$ round-bottom flask was charged with $\mathbf{I - 4}$, which was suspended in $\mathrm{Et}_{2} \mathrm{O}$. $\mathrm{HBArF}_{24} \cdot \mathrm{Et}_{2} \mathrm{O}^{15}$ was added and the reaction was stirred for 10 minutes, and 4 partitioned from $\mathrm{Et}_{2} \mathrm{O}$ as an oil. The layers were separated and the oil containing the bisamidinium salt was transferred to a round-bottom flask, which was placed under vacuum to yield a white foamy solid. The solid was triturated with hexanes $(\sim 20 \mathrm{~mL})$ and was dried under vacuum $(0.76 \mathrm{~g}, 48 \%$ yield $)$. Spectral data was identical to that which was previously reported.

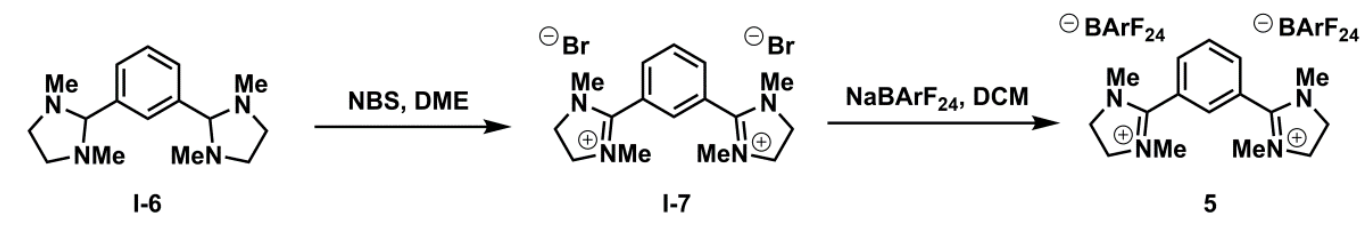

\section{2,2'-(1,3-phenylene)bis(1,3-dimethyl-4,5-dihydro-1H-imidazol-3-ium) tetrakis(3,5-}

\section{bis(trifluoromethyl)phenyl)borate (5)}

1,3-Bis(1,3-dimethylimidazolidine-2-yl)benzene (I-6) is a known compound and was synthesized as reported in the literature. ${ }^{21} \mathrm{~N}$-Bromosuccinimide (178 mg, $1.0 \mathrm{mmol}, 2.0$ equiv) was added in two portions to a solution of $\mathbf{I - 6}(137 \mathrm{mg}, 0.5 \mathrm{mmol}, 1.0$ equiv) in DME (3.3 $\mathrm{mL})$ at room temperature. A precipitate formed immediately and the reaction was stirred vigorously

${ }^{21}$ Corriu, R. J. P.; Mix, A.; Lanneau, G. F. J. Organomet. Chem. 1998, 570, 183-193. 
overnight. The reaction mixture was filtered to obtain a white solid that was washed with DME and dried under vacuum to yield the dibromide salt $\mathbf{I}-\mathbf{7}$, which was carried forward without further purification. $\mathrm{NaBArF}_{24}(88.6 \mathrm{mg}, 0.1 \mathrm{mmol}, 2.0$ equiv) was added in one portion to a solution of I-7 (21.6 mg, $0.05 \mathrm{mmol}, 1.0$ equiv) in DCM $(0.5 \mathrm{~mL})$ at room temperature. A white precipitate formed immediately. After stirring for 24 hours, the mixture was cooled to $0{ }^{\circ} \mathrm{C}$, filtered through Celite, and concentrated via rotary evaporation in vacuo to yield $\mathbf{5}$ as a white solid (95 mg, $0.48 \mathrm{mmol}, 95 \%$ yield).

${ }^{1}$ H NMR $\left(500 \mathrm{MHz}\right.$, Acetone- $\left.d_{6}\right) \delta 8.21(\mathrm{~s}, 1 \mathrm{H}), 8.18(\mathrm{~m}, 3 \mathrm{H}), 7.78(\mathrm{~s}, 16 \mathrm{H}), 7.67(\mathrm{~s}, 8 \mathrm{H}), 4.29$ (s, 8H), 3.19 (s, 12Hs);

${ }^{13} \mathbf{C}$ NMR $\left\{{ }^{1} \mathbf{H}\right\}\left(125 \mathrm{MHz}\right.$, Acetone- $\left.d_{6}\right) \delta 161.7$ (q, J=49.6 Hz), 134.6, 133.4, 131.8, 129.2, 129.1 (q, J=30.5 Hz), 124.5 (q, J=271.8 Hz), 124.0, 117.5, 50.8, 34.3;

IR (film) $v_{\max } 1701,1612,1354,1273,1113,887,839,745,712,682,670 \mathrm{~cm}^{-1}$;

MS (ESI-TOF) calcd. for $\mathrm{C}_{16} \mathrm{H}_{24} \mathrm{~N}_{4}\left[\mathrm{M}-\mathrm{BArF}_{24}\right] / 2$ 136.1000, found 136.0981 .

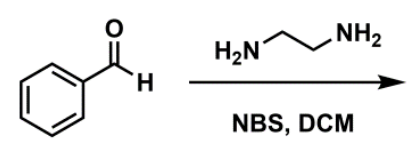

\section{4,5-dihydro-2-phenylimidazole (I-8)}

4,5-Dihydro-2-phenylimidazole ${ }^{22}$ is a known compound that was prepared by a different procedure as follows. To a solution of benzaldehyde ( $2.0 \mathrm{~g}, 18.8 \mathrm{mmol}, 1.0$ equiv.) in dichloromethane $(0.1 \mathrm{M})$ was added ethylenediamine. After stirring for $20 \mathrm{~min}$, the reaction mixture was cooled to $0{ }^{\circ} \mathrm{C}$ and $\mathrm{N}$-bromosuccinimide (3.35 g, $18.8 \mathrm{mmol}, 1.0$ equiv.) was added.

\footnotetext{
${ }^{22}$ Prokopcova, H.; Kappe, C. O. J. Org. Chem. 2007, 72, 4440-4448.
} 
The reaction mixture was allowed to warm to room temperature and was stirred overnight. Saturated aq. sodium bicarbonate was added and the reaction mixture was transferred to a separatory funnel. The aqueous phase was extracted with DCM $(3 \times 25 \mathrm{~mL})$ and the combined organics were washed with brine. The organic phase was dried over $\mathrm{Na}_{2} \mathrm{SO}_{4}$ and was concentrated in vacuo. The crude product was carried forward without further purification.

\section{4,5-dihydro-2-phenylimidazol-3-ium tetrakis(3,5-bis(trifluoromethyl)phenyl)borate (6)}

A solution of $\mathbf{I - 8}(0.2 \mathrm{~g}, 1.4 \mathrm{mmol}, 1.0$ equiv. $)$ in methanol $(0.5 \mathrm{M})$ was cooled to $0{ }^{\circ} \mathrm{C}$. $\mathrm{HCl}\left(1.4 \mathrm{~mL}, 2.0\right.$ equiv, $2 \mathrm{M}$ in $\left.\mathrm{Et}_{2} \mathrm{O}\right)$ was added dropwise. The reaction mixture was stirred for 5 minutes and was concentrated in vacuo. The residue was suspended in DCM (5 mL), $\operatorname{NaBArF}_{24}$ (1.2 g, $1.4 \mathrm{mmol}, 1.0$ equiv.) was added, and the mixture was stirred for 15 minutes. The reaction mixture was filtered through Celite, and the filtrate was concentrated in vacuo. Column chromatography on silica gel (5-10\% methanol in dichloromethane) afforded the product as a white solid (0.98 g, 69\% yield).

${ }^{1}$ H NMR $\left(500 \mathrm{MHz}\right.$, Acetone- $\left.d_{6}\right) \delta 8.01(\mathrm{~d}, \mathrm{~J}=7.0 \mathrm{~Hz}, 2 \mathrm{H}), 7.86-7.83(\mathrm{~m}, 1 \mathrm{H}), 7.79(\mathrm{~s}, 8 \mathrm{H})$, 7.70 (t, J = 7.8 Hz, 2 H), 7.68 (s, 4 H), 4.35 (s, 4 H);

${ }^{13} \mathbf{C}$ NMR $\left\{{ }^{1} \mathbf{H}\right\}\left(125 \mathrm{MHz}\right.$, Acetone- $\left.d_{6}\right) \delta 161.7$ (q, J=49.6 Hz) 135.0, 134.6, 129.6, 129.2, 129.0, $128.3,124.5$ (q, J=271.8 Hz), 122.3, 117.5, 45.1;

IR (film) $v_{\max } 3462.5,1607.2,1568.4,1353.4,1273.5,1113.1,1036.9,1001.2,985.2,935.9$, $888.0,838.7,779.3,744.5,712.6,695.9,681.4,670.7,617.3,544.8,450.2 \mathrm{~cm}^{-1}$;

MS (ESI-TOF) calcd. for [M - BAr $\left.{ }_{24}^{\mathrm{F}}\right]$ 147.0922, found 147.0902. 


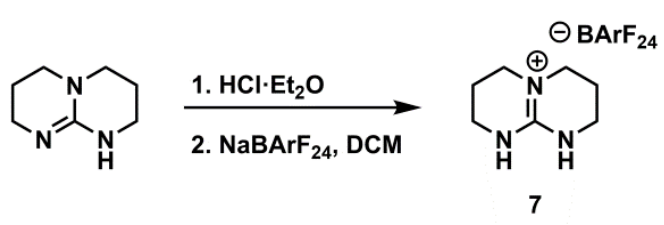

A solution of commercially available 1,5,7-triaazabicyclo[4.4.0]dec-5-ene $(0.51 \mathrm{~g}, 3.7$ mmol, 1.0 equiv.) in methanol was cooled to $0{ }^{\circ} \mathrm{C}$ and $\mathrm{HCl}\left(3.7 \mathrm{~mL}, 2.0 \mathrm{M}\right.$ in $\left.\mathrm{Et}_{2} \mathrm{O}\right)$ was added. The reaction mixture was stirred for 15 minutes and was concentrated in vacuo and the hydrochloride salt was isolated as a white solid. The hydrochloride salt $(0.25 \mathrm{~g}, 1.4 \mathrm{mmol}, 1.0$ equiv.) was suspended in dichloromethane and $\operatorname{NaBArF}_{24}(1.2 \mathrm{~g}, 1.4 \mathrm{mmol}, 1.0$ equiv.) was added. The reaction mixture was stirred for 15 minutes, was filtered through Celite and was concentrated in vacuo. Column chromatography on silica gel $(0-10 \%$ methanol in dichloromethane) afforded the product as a white solid ( $0.63 \mathrm{~g}, 45 \%$ yield $)$.

${ }^{1}$ H NMR (500 MHz, Acetone- $\left.d_{6}\right) \delta 7.79(\mathrm{~s}, 8 \mathrm{H}), 7.67(\mathrm{~s}, 4 \mathrm{H}), 7.04$ (br. s, $\left.2 \mathrm{H}\right), 3.51$ (t, J = 5.5 $\mathrm{Hz}, 4 \mathrm{H}), 3.42(\mathrm{t}, \mathrm{J}=5.5 \mathrm{~Hz}, 4 \mathrm{H}), 2.12-2.07$ (m, $4 \mathrm{H})$;

${ }^{13} \mathbf{C} \mathbf{N M R}\left\{{ }^{1} \mathbf{H}\right\}\left(125 \mathrm{MHz}\right.$, Acetone- $\left.d_{6}\right) \delta 161.6(\mathrm{q}, \mathrm{J}=49.8 \mathrm{~Hz}), 134.6,129.1(\mathrm{q}, \mathrm{J}=28.7 \mathrm{~Hz})$, $124.5(\mathrm{q}, \mathrm{J}=272.4 \mathrm{~Hz}), 117.5,46.8,38.2,20.5$;

IR (film) $v_{\max } 3343.5,1626.5,1355.1,1276.4,1121.4,887.1,838.9,713.2,682.3,670.1 \mathrm{~cm}^{-1}$; 


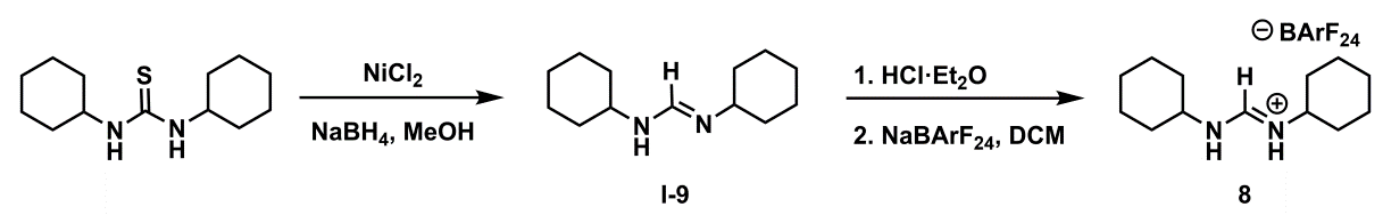

\section{$N, N^{\prime}$-dicyclohexylformamidinium tetrakis(3,5-bis(trifluoromethyl)phenyl)borate (8)}

N,N'-dicyclohexylformamidine (I-9) is a known compound that was prepared as previously reported. ${ }^{23}$ To a solution of $\mathbf{I - 9}$ in methanol was added $\mathrm{HCl}(1.87 \mathrm{~mL}, 2.0$ equiv, $2 \mathrm{M}$ in $\mathrm{Et}_{2} \mathrm{O}$ ), dropwise. The reaction mixture was stirred for 10 minutes and was concentrated via rotary evaporation in vacuo. The residue was suspended in dichloromethane and $\mathrm{NaBArF}_{24}(1.66$ $\mathrm{g}, 1.87 \mathrm{mmol}, 1.0$ equiv.) was added. The suspension was stirred for 15 minutes and a fine white precipitate formed, and the reaction mixture was then filtered through Celite. The filtrate was concentrated via rotary evaporation in vacuo to afford a colorless oil. Column chromatography on silica gel ( $0-5 \% \mathrm{MeOH}$ in $\mathrm{DCM})$ yielded the product as a white solid $(0.52 \mathrm{~g}, 26 \%$ yield).

${ }^{1}$ H NMR (500 MHz, Acetone- $d_{6}$ ) $\delta 8.70$ (br. s., 2 H), 8.16 (s, 1 H), 7.79 (s, 8 H), 7.67 (s, 4 H), 3.76-3.74 (m, $1 \mathrm{H}), 3.64-3.61(\mathrm{~m}, 1 \mathrm{H}), 2.05-2.02(\mathrm{~m}, 4 \mathrm{H}), 1.78-1.61(\mathrm{~m}, 6 \mathrm{H}), 1.49-1.12(\mathrm{~m}$, $10 \mathrm{H})$;

${ }^{13} \mathbf{C} \mathbf{N M R}\left\{{ }^{1} \mathbf{H}\right\}\left(125 \mathrm{MHz}\right.$, Acetone- $\left.d_{6}\right) \delta 161.6(\mathrm{q}, \mathrm{J}=49.8 \mathrm{~Hz}), 152.5,134.6,129.1$ (q, J=28.7 $\mathrm{Hz}), 124.5$ (q, J=272.4 Hz), 117.5, 51.2, 33.0, 24.6, 24.1;

IR (film) $v_{\max } 2943.7,2864.5,1697.8,1610.2,1538.0,1454.9,1353.0,1272.7,1114.4,931.1$, $886.3,838.2,744.3,712.1,681.4,669.3,597.1,582.5,448.5 \mathrm{~cm}^{-1}$;

MS (ESI-TOF) calcd. for [M - BAr $\left.{ }_{24}^{\mathrm{F}}\right]$ 209.2018, found 209.2045.

\footnotetext{
${ }^{23}$ Khurana, J. M.; Kukrega, G.; Bansal, G. J. Chem. Soc., Perkin Trans 1 2002, 22, 2520-2524.
} 


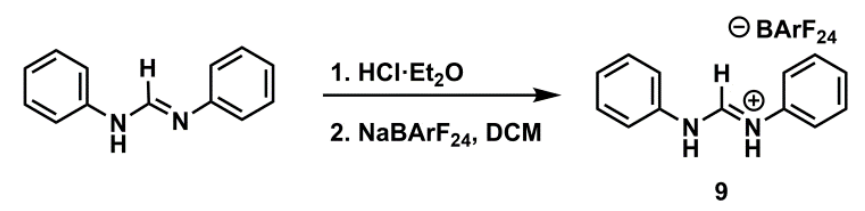

A solution of commercially available diphenylformamidine ( $1 \mathrm{~g}, 5.1 \mathrm{mmol}, 1.0$ equiv.) in 1:1 THF: $\mathrm{Et}_{2} \mathrm{O}$ was cooled to $0{ }^{\circ} \mathrm{C}$. $\mathrm{HCl}\left(5.1 \mathrm{~mL}, 2.0 \mathrm{M}^{\text {in }} \mathrm{Et}_{2} \mathrm{O}\right)$ was added and the solution was stirred for $30 \mathrm{~min}$. The hydrochloride salt precipitated as a white solid and was isolated via filtration. The hydrochloride salt (184 mg, $0.79 \mathrm{mmol}, 1.0$ equiv.) was suspended in DCM and $\mathrm{NaBArF}_{24}(0.7 \mathrm{~g}, 0.79 \mathrm{mmol}, 1.0$ equiv.) was added. The reaction mixture was stirred for $15 \mathrm{~min}$, was filtered through Celite and concentrated in vacuo to isolate 9 as a tan solid $(0.65 \mathrm{~g}, 78 \%$ yield).

${ }^{1}$ H NMR $\left(500 \mathrm{MHz}\right.$, Acetone- $\left.d_{6}\right) \delta 9.36(\mathrm{~s}, 1 \mathrm{H}), 7.80(\mathrm{~s}, 8 \mathrm{H}), 7.68(\mathrm{~s}, 4 \mathrm{H}), 7.63-7.41(\mathrm{~m}, 10 \mathrm{H})$;

${ }^{13} \mathbf{C} \mathbf{N M R}\left\{{ }^{1} \mathbf{H}\right\}\left(125 \mathrm{MHz}\right.$, Acetone- $\left.d_{6}\right) \delta 161.6$ (q, J=49.8 Hz), 134.6, 129.8, 129.1 (q, J=28.7 $\mathrm{Hz}), 127.5,124.5$ (q, J=272.4 Hz), 119.9, 117.5;

IR (film) $v_{\max } 1694.0,1595.9,1499.7,1352.9,1272.8,1113.9,1027.7,930.8,886.3,838.0$, $753.8,744.4,712.2,681.4,669.5,579.7,504.9,448.5 \mathrm{~cm}^{-1}$

MS (ESI-TOF) calcd. for [M - BAr $\left.{ }_{24}^{\mathrm{F}}\right]$ 197.1079, found 197.1087

\section{2a.7.3 Cyclic voltammetry experiments}

\section{General information}

Cyclic voltammetry measurements were performed with a $\mathrm{CH}$ Instruments (Austin, TX) model 610D electrochemical workstation using CHI 610D software. All experiments were conducted in a glovebox under an atmosphere of $\mathrm{N}_{2}$, using a $\mathrm{CH}$ instruments glassy carbon working electrode $\left(\right.$ area $=0.076 \mathrm{~cm}^{2}$ ), a Pt wire counter electrode, and Ag wire in $0.1 \mathrm{M}$ $n \mathrm{Bu}_{4} \mathrm{NBArF}_{24} / \mathrm{DCM}$ as reference electrode. DCM and THF were purified by passing through a 
column of activated alumina and were stored in the glovebox. All stock solutions were prepared in the glove box in volumetric flasks. All CVs were recorded with compensation for solution resistance and were referenced to the ferrocenium/ferrocene $\left(\mathrm{Fc}^{+/ 0}\right)$ couple by recording the $\mathrm{CVs}$ in the presence of ferrocene after each titration was complete. Solutions were stirred between acquisition of individual CVs and the working electrode was polished between titration experiments. Simulations of the CV experiments were performed using the DigiElch V. $7 .^{24}$ Symmetry factors ( $\alpha$ values) were set as 0.5 for all electron transfer steps.

Tetrabutylammonium $\mathrm{BArF}_{24}(0.1 \mathrm{M}, 4 \mathrm{~mL}$ total volume) was used as the electrolyte, and was prepared by mixing $n \mathrm{Bu} 4 \mathrm{NCl}$ and $\mathrm{NaBArF}_{24}$ in dichloromethane, filtering through Celite, and concentrating in vacuo. Recrystallization of the solid residue from pentane/Et $\mathrm{t}_{2} \mathrm{O}$ afforded the product as colorless needles.

Discrepancies in current magnitude between experiment and simulation are noted in many data sets. This is attributed to variations in the diffusion coefficient (D) between the complexes that are relevant to the simulation. D was assumed to be constant ${ }^{25}$ and was fixed at 1 $\mathrm{x} 10^{-5} \mathrm{~cm}^{2} / \mathrm{s}$ for all of the species involved. However, D is likely to change for the various complexes involving $\mathbf{Q}$, due to changes in molecular mass. Perturbing the $\mathrm{D}$ values within the simulations indicates that this can account for the differences in current magnitude. However, due to the number of parameters involved in each simulation, determination of precise $\mathrm{D}$ values for each relevant species was not pursued.

\footnotetext{
${ }^{24}$ Rudolf, M. J. Electroanal. Chem. 2003, 543, 23-39. Digital simulations on unequally spaced grids.: Part 2. Using the box method by discretisation on a transformed equally spaced grid. DigiElch from Elchsoft under http://www.elchsoft.com.

${ }^{25}$ This is a commonly applied assumption in simulation analysis of electrochemical data. For examples, see: (a) Ge, Y.; Miller, L.; Ouimet, T.; Smith, D. K. J. Org. Chem. 2000, 65, 8831-8838. (b) Bu, J.; Lilienthal, N. D.; Woods, J. E.; Nohrden, C. E.; Hoang, K. T.; Truong, D.; Smith, D. K. J. Am. Chem. Soc. 2005, 127, 6423-6429. (c) Chan-Leonor, C.; Martin, S. L.; Smith, D. K. J. Org. Chem. 2005, 70, 1081710822
} 


\section{CV experiments with 1}

A $0.1 \mathrm{M}$ stock solution of $\mathbf{1}(108.6 \mathrm{mg})$ in $\mathrm{CH}_{2} \mathrm{Cl}_{2}$ was prepared in a $1 \mathrm{~mL}$ volumetric flask. A stock solution of $o$-chloranil $\left(6.4 \mathrm{mg}\right.$ in $\left.0.1 \mathrm{M} \mathrm{nBu} \mathrm{BBArF}_{24} / \mathrm{CH}_{2} \mathrm{Cl}_{2}\right)$ was prepared in a $1 \mathrm{~mL}$ volumetric flask. A $0.5 \mathrm{mM}$ solution of $o$-chloranil in $0.1 \mathrm{M} \mathrm{nBu} \mathrm{NBArF}_{24} / \mathrm{CH}_{2} \mathrm{Cl}_{2}$ was prepared in an electrochemical cell using $77 \mu \mathrm{L}$ of the $o$-chloranil stock solution and $3.9 \mathrm{~mL}$ of $0.1 \mathrm{M} n \mathrm{Bu}_{4} \mathrm{NBArF}_{24} / \mathrm{CH}_{2} \mathrm{Cl}_{2}$. The $\mathrm{CV}$ of the solution was recorded. The $o$-chloranil solution was titrated with $10-200 \mu \mathrm{L}$ increments of $\mathbf{1}$, and a CV was recorded after each addition of $\mathbf{1}$. The experimental data were reproduced by simulation of a CEC mechanism.

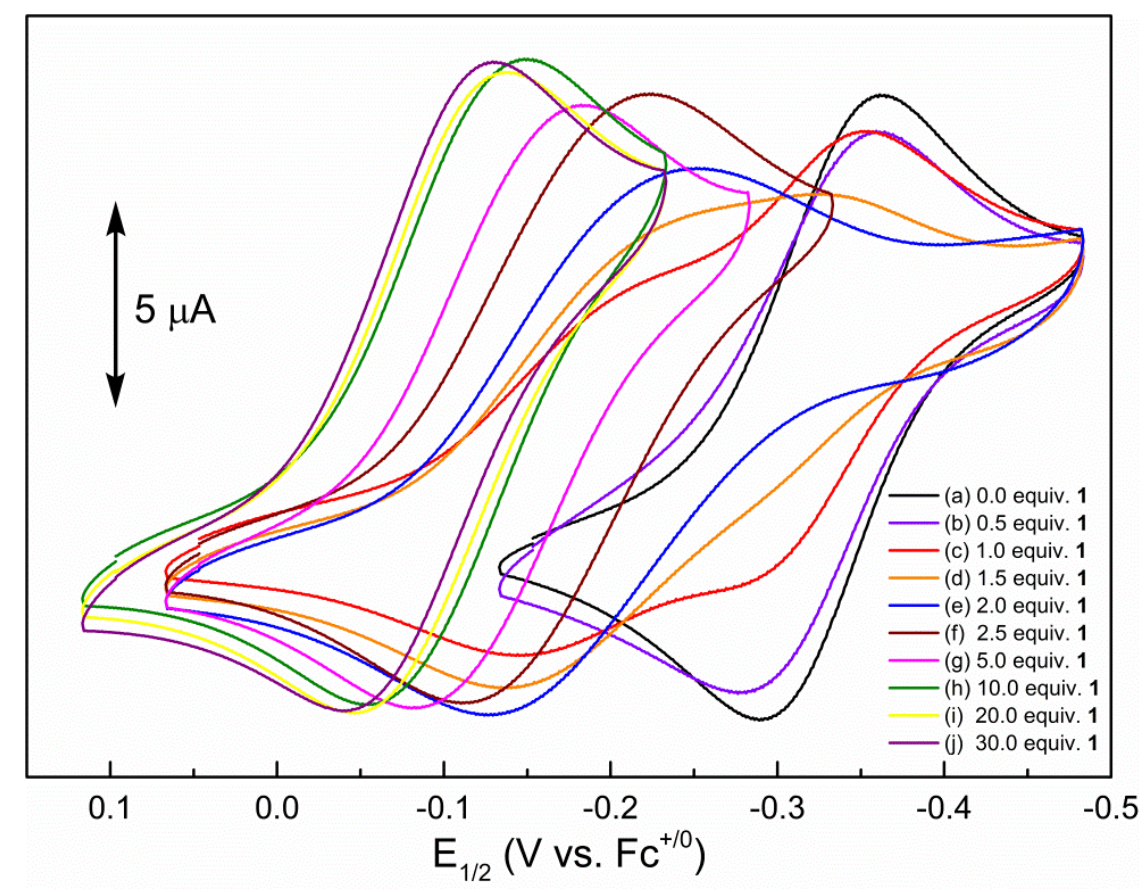

Figure 2.32. Full set of experimental CVs of $o$-chloranil (initial concentration $=0.5 \mathrm{mM}$ ) recorded in $0.1 \mathrm{M} n \mathrm{Bu}_{4} \mathrm{NBArF}_{24} / \mathrm{CH}_{2} \mathrm{Cl}_{2}$ (glovebox) in the presence of increasing [1]. 


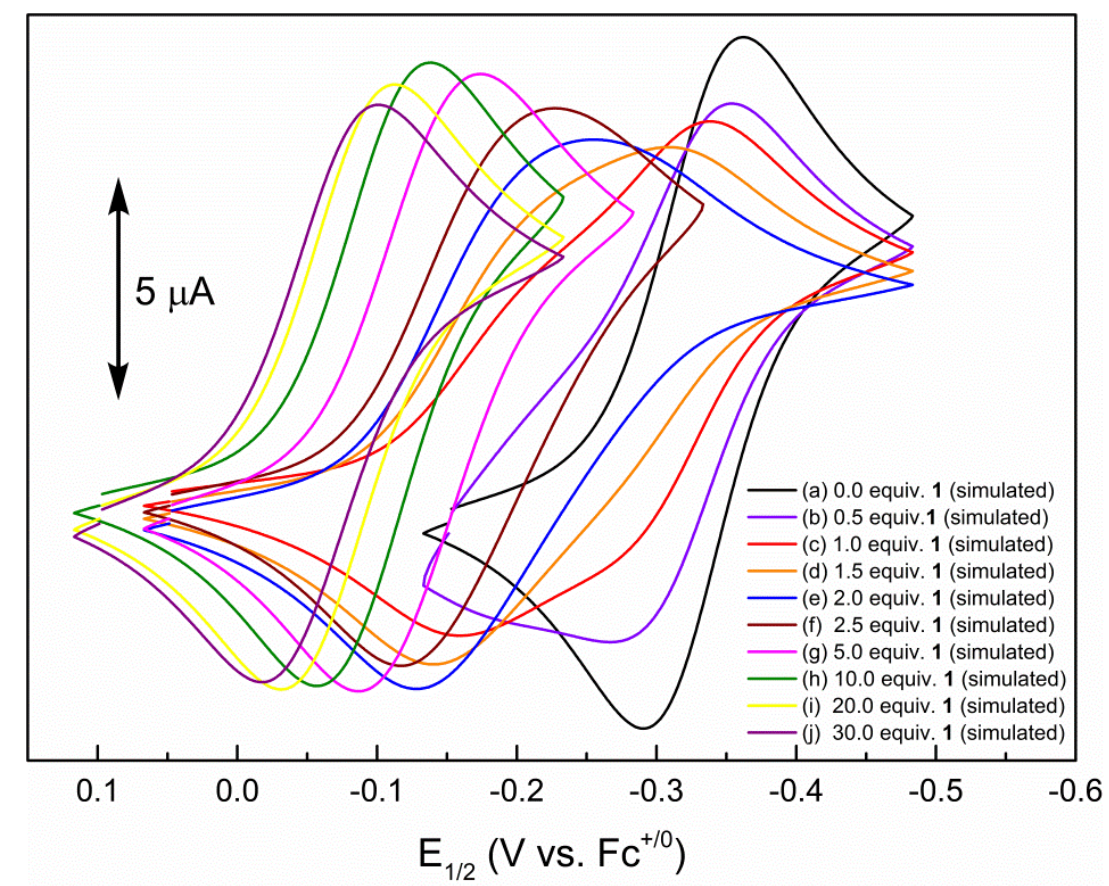

Figure 2.33. Full set of CEC simulations of $\mathrm{CV}$ data for $o$-chloranil (initial concentration $=0.5$ $\mathrm{mM}$ ) recorded in $0.1 \mathrm{MnBu} \mathrm{NBArF}_{24} / \mathrm{CH}_{2} \mathrm{Cl}_{2}$ (glovebox) in the presence of increasing [1].

Simulations of a CE mechanism and ECC mechanism both provided poor fits to the experimental data. Figure 2.34 shows the comparison of the CE simulation with the experimental data. The irregular curve shapes at low [1] are not reproduced by this mechanism (e.g. scan (b) and simulation (f)), nor is the overall $\Delta \mathrm{E}_{1 / 2}($ e.g. scan (d) and simulation (h)). 


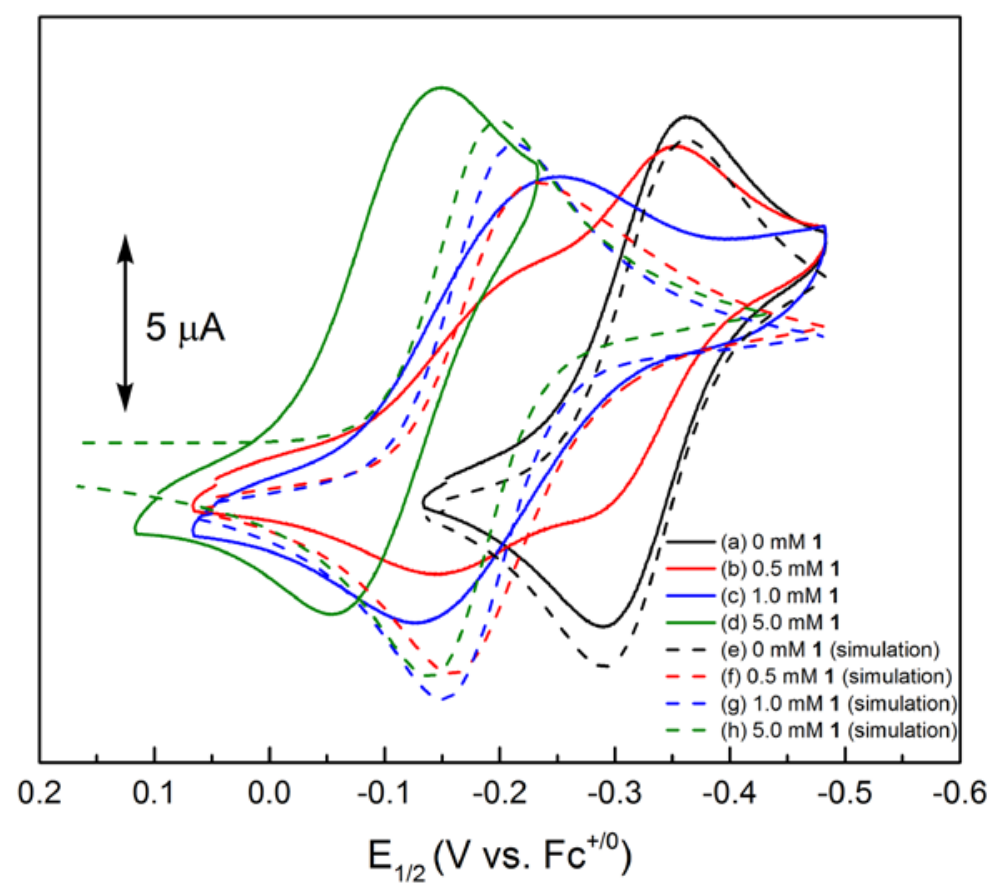

Figure 2.34. Experimental CVs of $o$-chloranil (initial concentration $=0.5 \mathrm{mM}$ ) recorded in 0.1 $\mathrm{M} n \mathrm{Bu}_{4} \mathrm{NBArF}_{24} / \mathrm{CH}_{2} \mathrm{Cl}_{2}$ (glovebox) in the presence of increasing [1] and comparison with simulation of a $\mathrm{CE}$ mechanism representing a poor fit.

Figure 2.35 compares the simulation of an ECC mechanism with the experimental data. While this mechanism does provide a good fit to the data collected at low [1] (e.g. scan (b) and simulation (f)) and does reasonably reproduce the overall $\Delta \mathrm{E}_{1 / 2}$, the reversibility observed experimentally at large [1] is inconsistent with the irreversibility obtained by the simulation (e.g scan (e) and simulation (j)). 


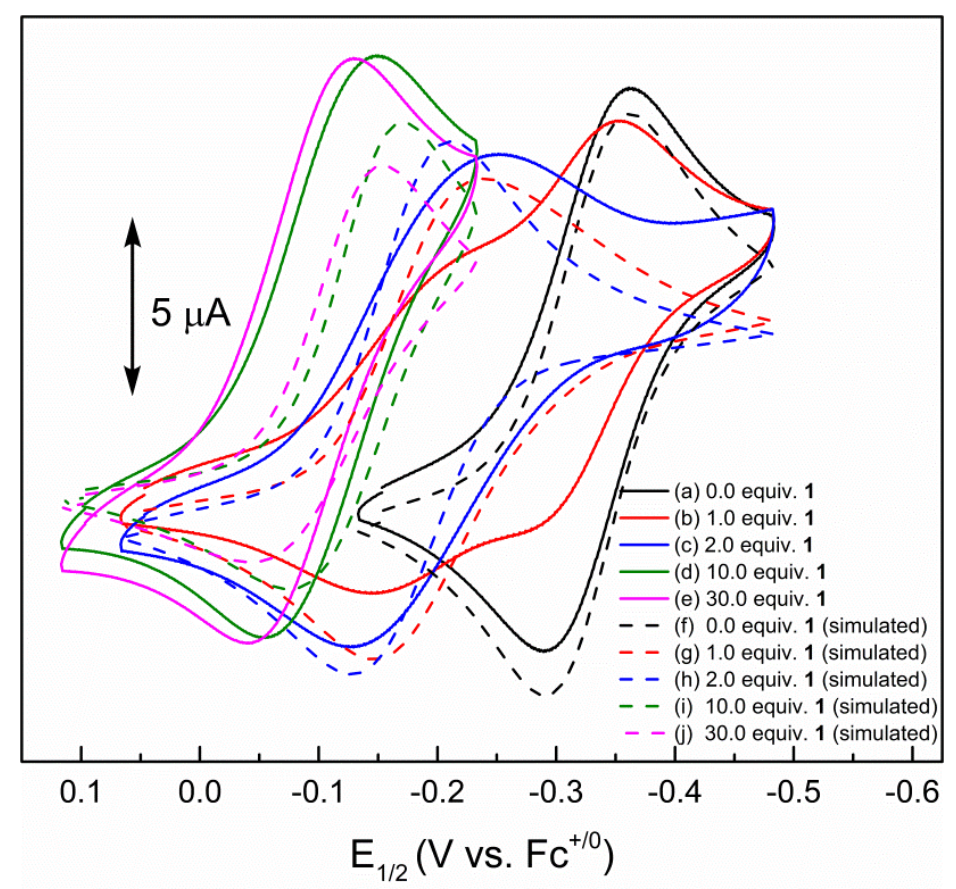

Figure 2.35. Experimental $\mathrm{CVs}$ of $o$-chloranil (initial concentration $=0.5 \mathrm{mM}$ ) recorded in 0.1 $\mathrm{M} n \mathrm{Bu}_{4} \mathrm{NBArF}_{24} / \mathrm{CH}_{2} \mathrm{Cl}_{2}$ (glovebox) in the presence of increasing [1] and comparison with simulation of an ECC mechanism representing a poor fit.

\section{Cyclic voltammetry experiments with 2}

A $0.1 \mathrm{M}$ stock solution of $2(107.7 \mathrm{mg})$ in $\mathrm{CH}_{2} \mathrm{Cl}_{2}$ was prepared in a $1 \mathrm{~mL}$ volumetric flask. A stock solution of $o$-chloranil $\left(6.4 \mathrm{mg}\right.$ in $\left.0.1 \mathrm{M} n \mathrm{Bu}_{4} \mathrm{NBArF}_{24} / \mathrm{CH}_{2} \mathrm{Cl}_{2}\right)$ was prepared in a $1 \mathrm{~mL}$ volumetric flask. A $0.5 \mathrm{mM}$ solution of $o$-chloranil in $0.1 \mathrm{M} \mathrm{nBu} \mathrm{NBArF}_{24} / \mathrm{CH}_{2} \mathrm{Cl}_{2}$ was prepared in an electrochemical cell using $77 \mu \mathrm{L}$ of the $o$-chloranil stock solution and $3.9 \mathrm{~mL}$ of 0.1 $\mathrm{M} n \mathrm{Bu}_{4} \mathrm{NBArF}_{24} / \mathrm{CH}_{2} \mathrm{Cl}_{2}$. The $\mathrm{CV}$ of the solution was recorded. The $o$-chloranil solution was titrated with 10-200 $\mu \mathrm{L}$ increments of $\mathbf{2}$, and a CV was recorded after each addition of $\mathbf{2}$. The experimental data were reproduced by simulation of a CEC mechanism. 


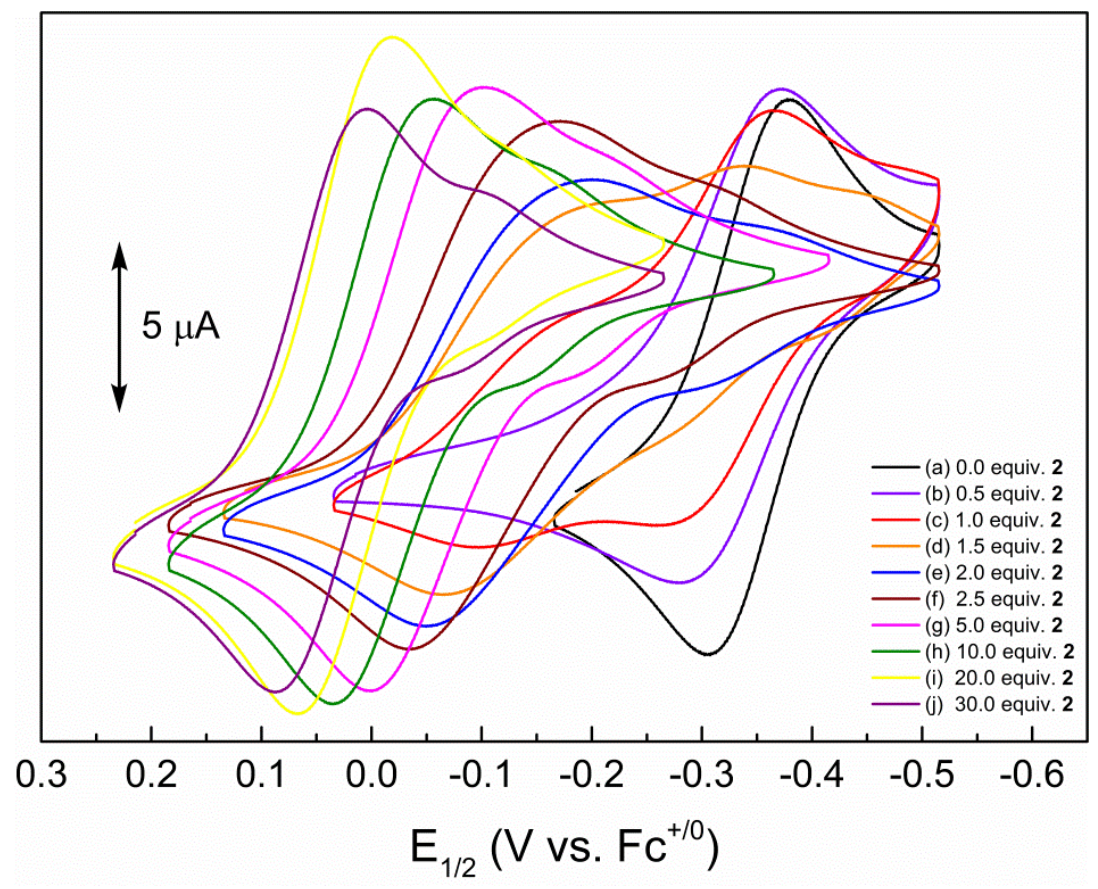

Figure 2.36. Full set of experimental CVs of $o$-chloranil (initial concentration $=0.5 \mathrm{mM}$ ) recorded in $0.1 \mathrm{M} \mathrm{nBu} \mathrm{BuArF}_{24} / \mathrm{CH}_{2} \mathrm{Cl}_{2}$ (glovebox) in the presence of increasing [2]. In scans (d)-(j), the additional current peaks at lower potential are attributed to the $2 \mathrm{e}^{-}$reduction.

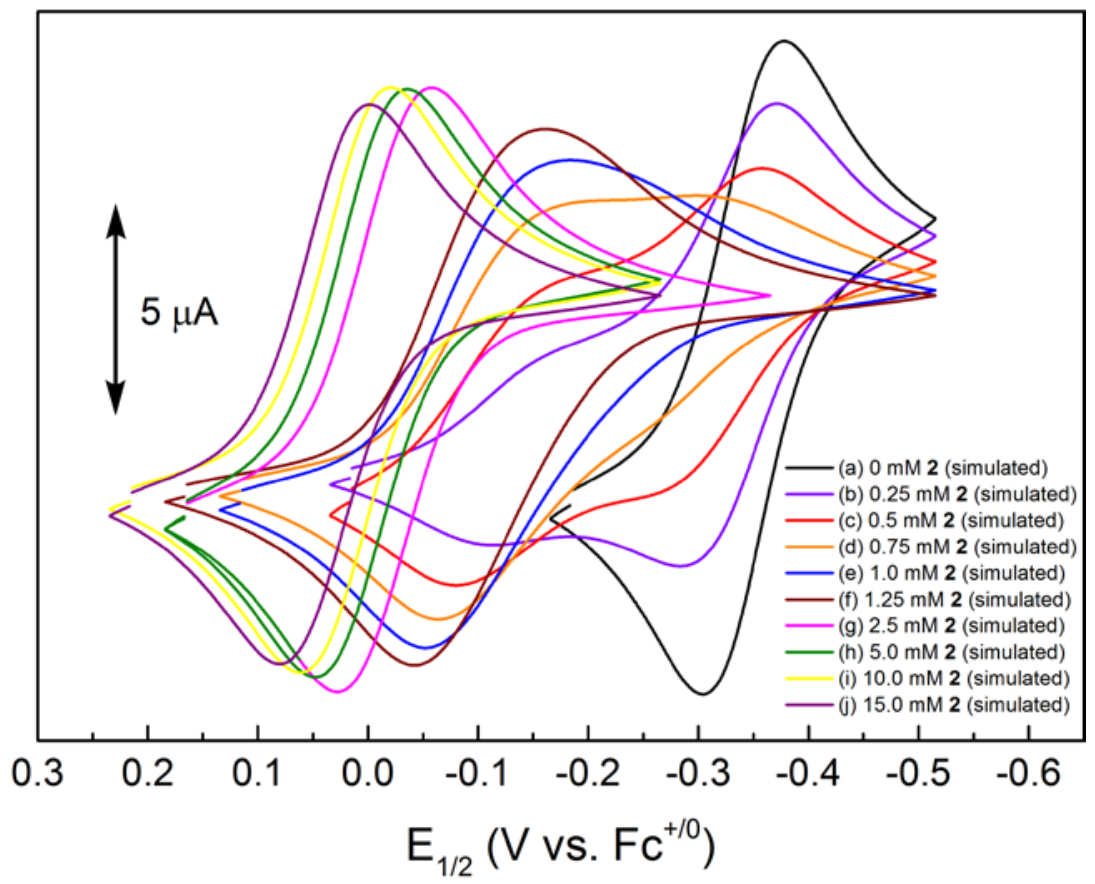

Figure 2.37. Full set of $\mathrm{CEC}$ simulations of $\mathrm{CV}$ data for $o$-chloranil (initial concentration $=0.5$ $\mathrm{mM}$ ) recorded in $0.1 \mathrm{M} \mathrm{n} n \mathrm{Bu}_{4} \mathrm{NBArF}_{24} / \mathrm{CH}_{2} \mathrm{Cl}_{2}$ (glovebox) in the presence of increasing [2]. 


\section{CV experiments with 3}

A $0.1 \mathrm{M}$ stock solution of $3(65.1 \mathrm{mg})$ in $10 \% \mathrm{THF} / \mathrm{CH}_{2} \mathrm{Cl}_{2}$ was prepared in a $1 \mathrm{~mL}$ volumetric flask. The addition of THF was necessary to fully dissolve 3. Data were also obtained with 1 using a stock solution in $10 \% \mathrm{THF} / \mathrm{CH}_{2} \mathrm{Cl}_{2}$, and the presence of THF in these small quantities was not found to have an impact on the measured equilibrium constants. A stock solution of $o$-chloranil $\left(9.8 \mathrm{mg}\right.$ in $0.1 \mathrm{M} \mathrm{nBu} \mathrm{NBArF}_{24} / \mathrm{CH}_{2} \mathrm{Cl}_{2}$ ) was prepared in a $1 \mathrm{~mL}$ volumetric flask. A $0.5 \mathrm{mM}$ solution of $o$-chloranil in $0.1 \mathrm{M} n \mathrm{Bu}_{4} \mathrm{NBArF}_{24} / \mathrm{CH}_{2} \mathrm{Cl}_{2}$ was prepared in an electrochemical cell using $50 \mu \mathrm{L}$ of the $o$-chloranil stock solution and $3.95 \mathrm{~mL}$ of $0.1 \mathrm{M}$ $n \mathrm{Bu}_{4} \mathrm{NBArF}_{24} / \mathrm{CH}_{2} \mathrm{Cl}_{2}$. The $o$-chloranil solution was titrated with $10-200 \mu \mathrm{L}$ increments of $\mathbf{3}$, and a $\mathrm{CV}$ was recorded after each addition of $\mathbf{3}$. The experimental data were reproduced by simulation of a CEC mechanism.

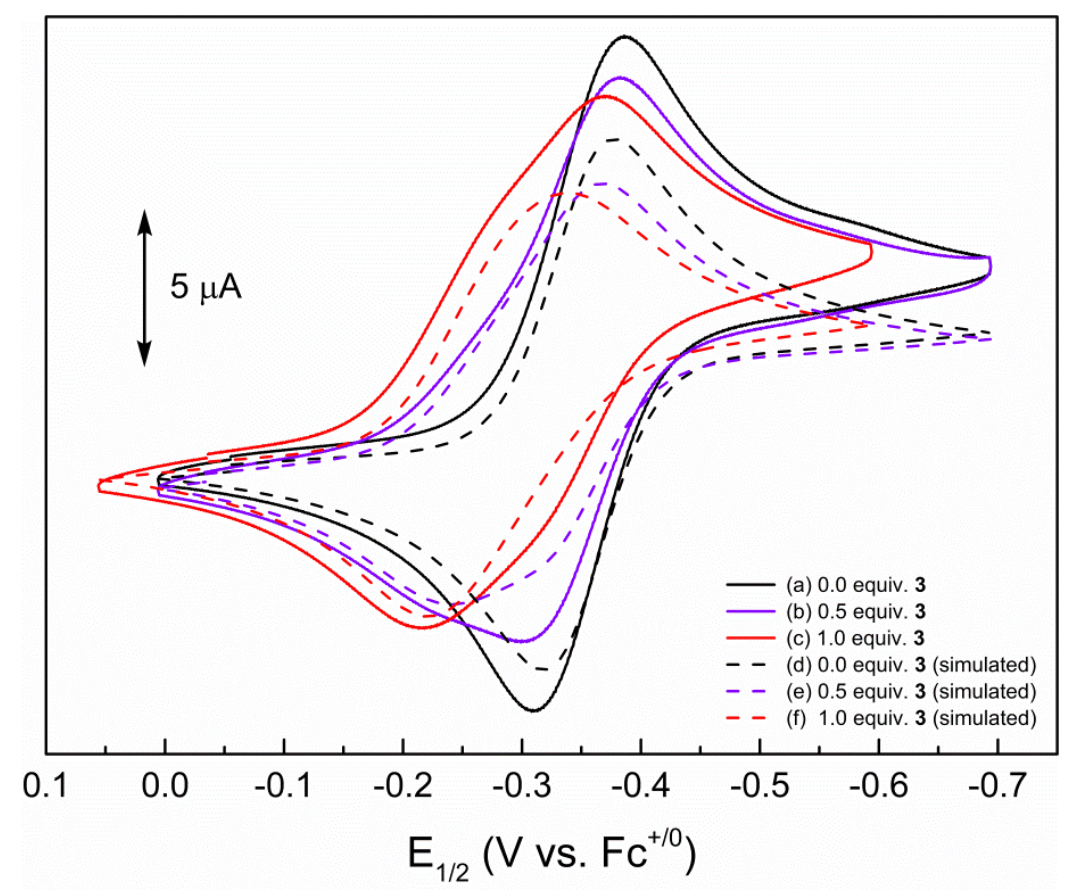

Figure 2.38. Experimental CVs of $o$-chloranil (initial concentration $=0.5 \mathrm{mM}$ ) recorded in 0.1 $\mathrm{M} n \mathrm{Bu}_{4} \mathrm{NBArF}_{24} / \mathrm{CH}_{2} \mathrm{Cl}_{2}$ (glovebox) in the presence of increasing [3] and comparison with simulation of a CEC mechanism. Only data collected at low [3] are shown. The simulated scan (e) replicates the irregular curve shape observed experimentally in scan (b), and simulated scan (f) partially replicates the broadness observed experimentally in scan (c). 


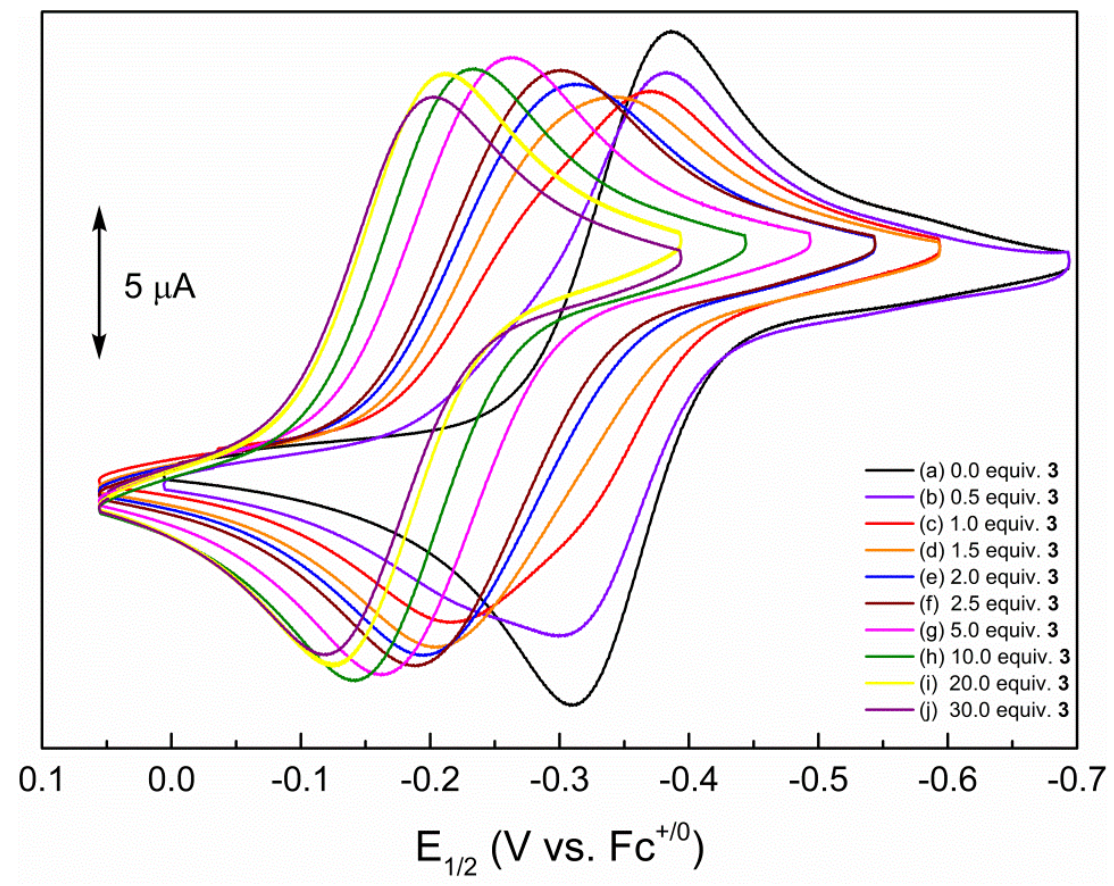

Figure 2.39. Full set of experimental $\mathrm{CVs}$ of $o$-chloranil (initial concentration $=0.5 \mathrm{mM}$ ) recorded in $0.1 \mathrm{M} n \mathrm{Bu}_{4} \mathrm{NBArF}_{24} / \mathrm{CH}_{2} \mathrm{Cl}_{2}$ (glovebox) in the presence of increasing [3].

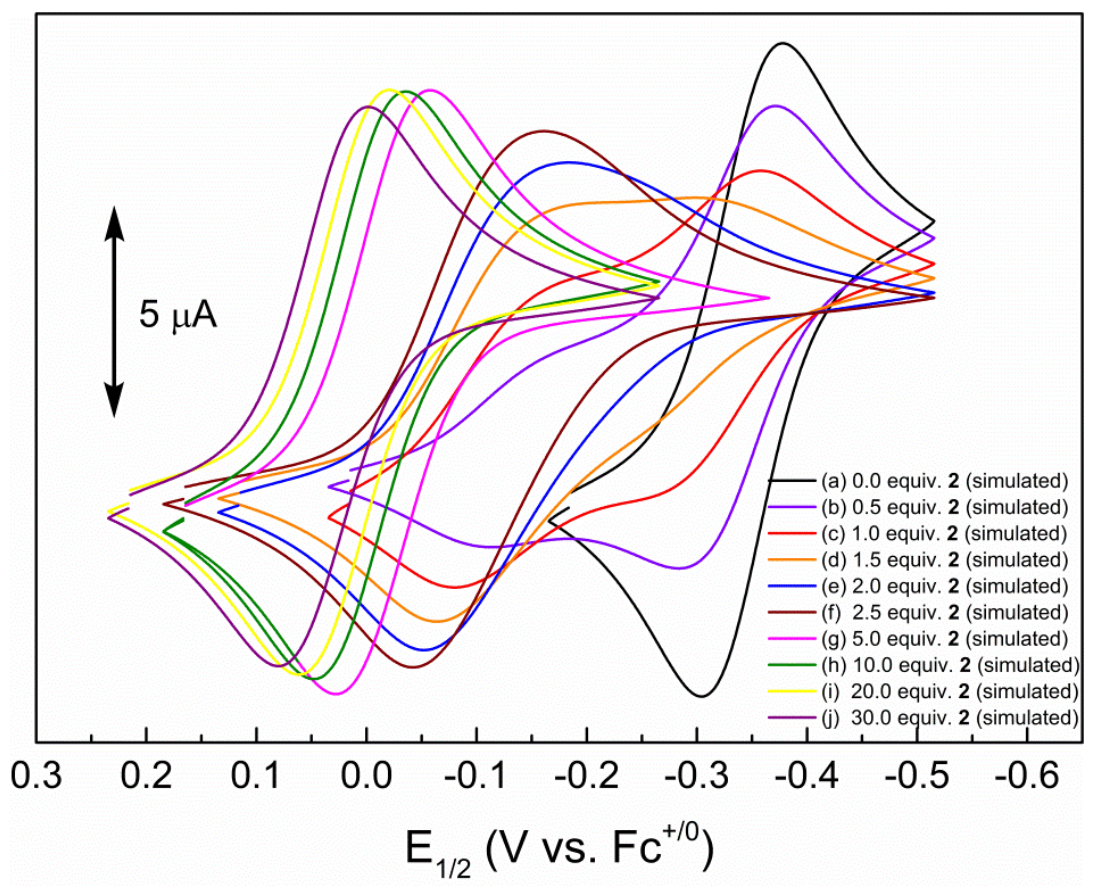

Figure 2.40. Full set of simulations of $\mathrm{CV}$ data for $o$-chloranil (initial concentration $=0.5 \mathrm{mM}$ ) recorded in $0.1 \mathrm{M} \mathrm{nBu} \mathrm{NBArF}_{24} / \mathrm{CH}_{2} \mathrm{Cl}_{2}$ (glovebox) in the presence of increasing [3]. 


\section{CV experiments with 4}

A $0.05 \mathrm{M}$ stock solution of $4(200.1 \mathrm{mg})$ in $\mathrm{CH}_{2} \mathrm{Cl}_{2}$ was prepared in a $2 \mathrm{~mL}$ volumetric flask. A stock solution of $o$-chloranil $\left(5.0 \mathrm{mg}\right.$ in $\left.0.1 \mathrm{M} n \mathrm{Bu}_{4} \mathrm{NBArF}_{24} / \mathrm{CH}_{2} \mathrm{Cl}_{2}\right)$ was prepared in a $2 \mathrm{~mL}$ volumetric flask. A $0.5 \mathrm{mM}$ solution of $o$-chloranil in $0.1 \mathrm{M} n \mathrm{Bu}_{4} \mathrm{NBArF}_{24} / \mathrm{CH}_{2} \mathrm{Cl}_{2}$ was prepared in an electrochemical cell using $197 \mu \mathrm{L}$ of the $o$-chloranil stock solution and $3.8 \mathrm{~mL}$ of $0.1 \mathrm{M} n \mathrm{Bu}_{4} \mathrm{NBArF}_{24} / \mathrm{CH}_{2} \mathrm{Cl}_{2}$. The $\mathrm{CV}$ of the solution was recorded. The $o$-chloranil solution was titrated with $20-40 \mu \mathrm{L}$ increments of 4 , and a $\mathrm{CV}$ was recorded after each addition of 4 . The experimental data were reasonably reproduced by simulation of a CE mechanism.

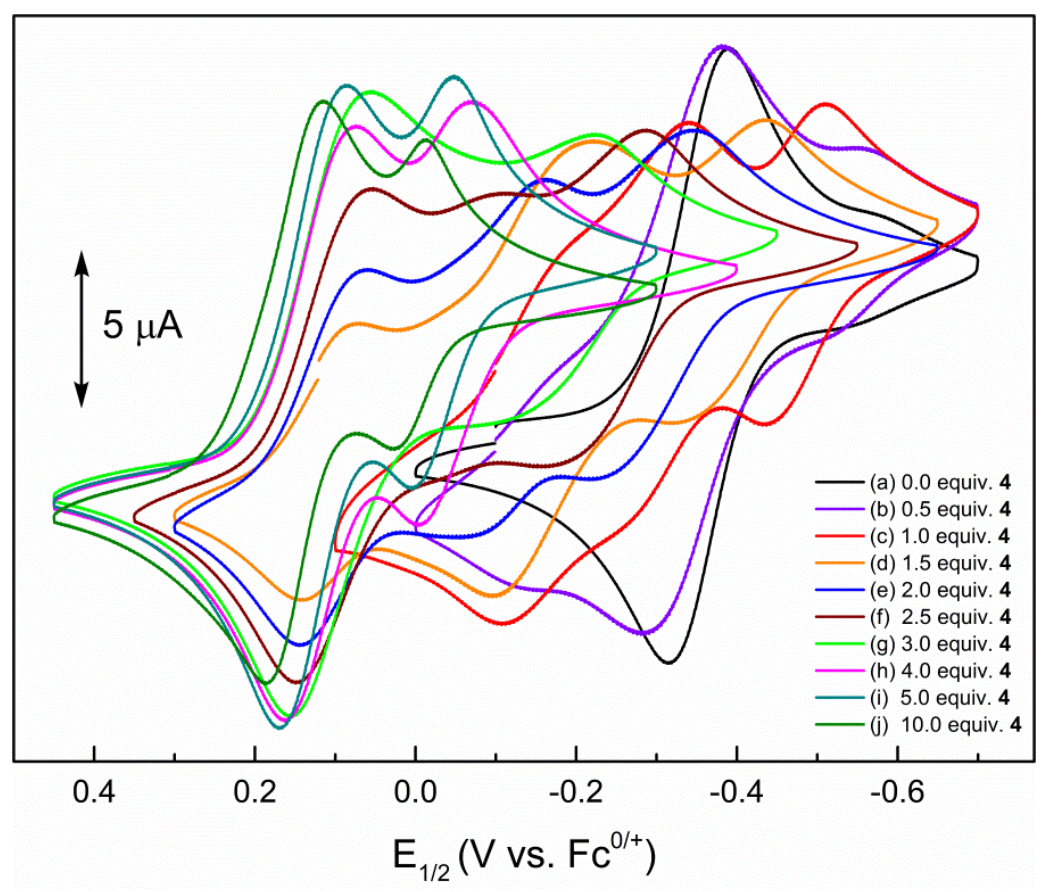

Figure 2.41. Full set of experimental $\mathrm{CVs}$ of $0.5 \mathrm{mM} o$-chloranil recorded in $0.1 \mathrm{M}$ $n \mathrm{Bu}_{4} \mathrm{NBArF}_{24} / \mathrm{CH}_{2} \mathrm{Cl}_{2}$ (glovebox) in the presence of increasing [4]. In scans (b)- $(\mathrm{j})$, the additional current peaks at lower potential are attributed to the $2 \mathrm{e}^{-}$reduction. 


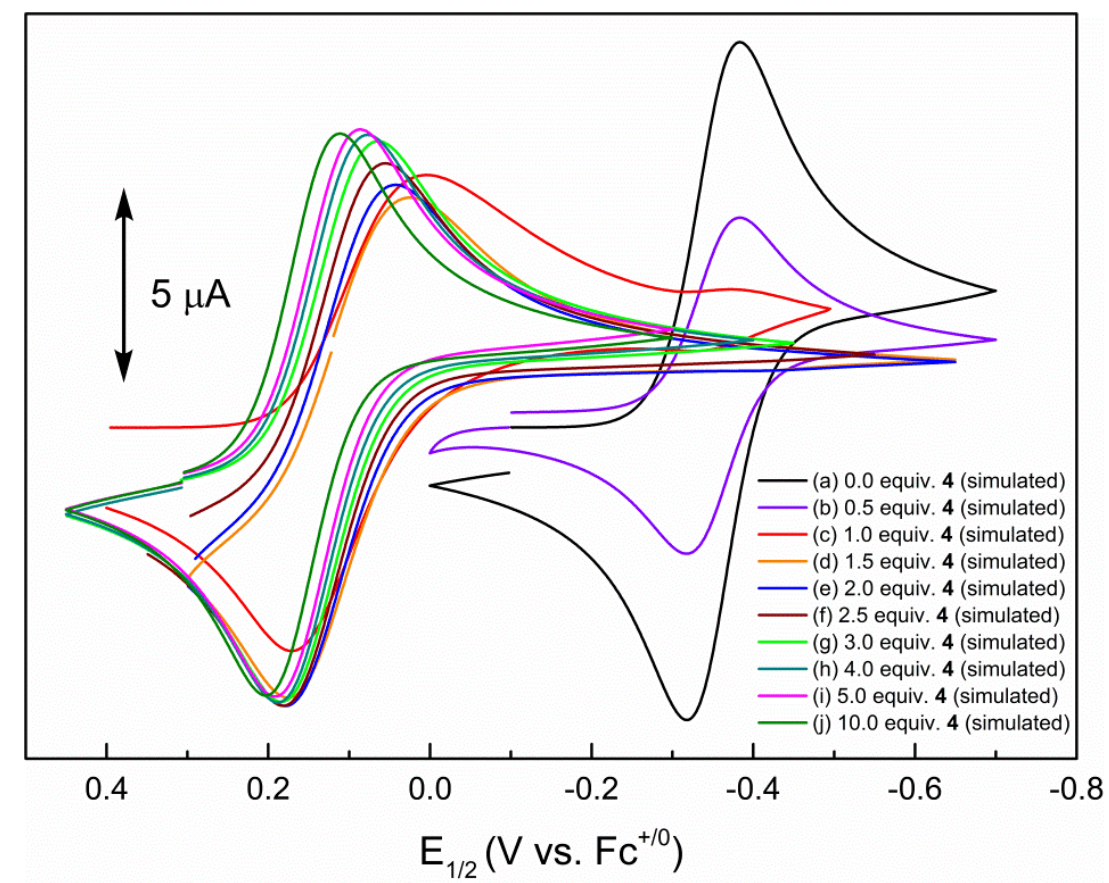

Figure 2.42. Full set of simulations of $\mathrm{CV}$ data for $o$-chloranil (initial concentration $=0.5 \mathrm{mM}$ ) recorded in $0.1 \mathrm{Mn} n \mathrm{Bu}_{4} \mathrm{NBArF}_{24} / \mathrm{CH}_{2} \mathrm{Cl}_{2}$ (glovebox) in the presence of increasing [4]

Simulations of an EC mechanism provided a poor fit to the experimental data (Figure 2.42). The overall $\Delta \mathrm{E}_{1 / 2}$ is not reproduced - even with very large values for $\mathrm{K}_{3}$ (in this simulation, $\mathrm{K}_{3}=1 \times 10^{14} \mathrm{M}^{-1}$ ), the $\mathrm{CV}$ waves are never shifted to the same extent as in the experimental data. Furthermore, the simulated CV waves are irreversible, which is inconsistent with the reversibility that is observed over the course of the titration. 


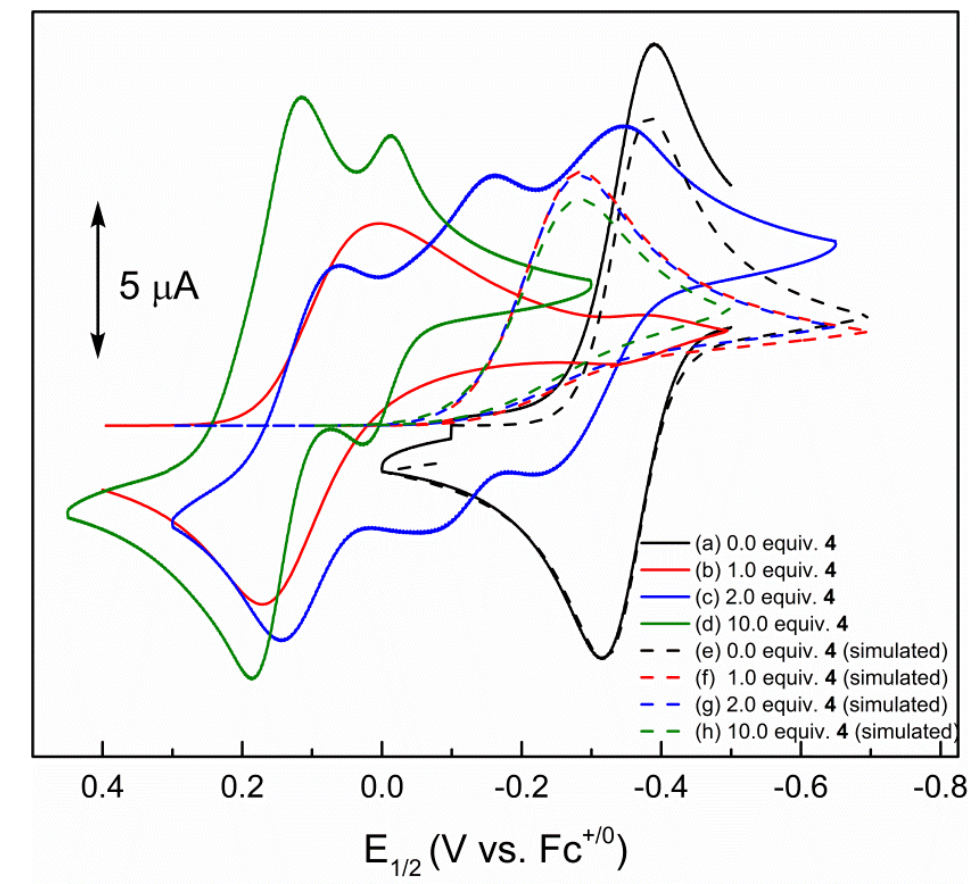

Figure 2.43. Experimental CVs of $o$-chloranil (initial concentration $=0.5 \mathrm{mM}$ ) recorded in 0.1 $\mathrm{M} n \mathrm{Bu}_{4} \mathrm{NBArF}_{24} / \mathrm{CH}_{2} \mathrm{Cl}_{2}$ (glovebox) in the presence of increasing [4] and comparison with simulation of an $\mathrm{EC}$ mechanism representing a poor fit.

\section{CV experiments with 5}

A $0.05 \mathrm{M}$ stock solution of $5(198.2 \mathrm{mg})$ in $\mathrm{CH}_{2} \mathrm{Cl}_{2}$ was prepared in a $2 \mathrm{~mL}$ volumetric flask. A stock solution of $o$-chloranil (4.1 mg in $0.1 \mathrm{M} n \mathrm{Bu}_{4} \mathrm{NBArF}_{24} / \mathrm{CH}_{2} \mathrm{Cl}_{2}$ ) was prepared in a $1 \mathrm{~mL}$ volumetric flask. A $0.5 \mathrm{mM}$ solution of $o$-chloranil in $0.1 \mathrm{M} \mathrm{nBu} \mathrm{NBArF}_{24} / \mathrm{CH}_{2} \mathrm{Cl}_{2}$ was prepared in an electrochemical cell using $120 \mu \mathrm{L}$ of the $o$-chloranil stock solution and $3.9 \mathrm{~mL}$ of $0.1 \mathrm{M} n \mathrm{Bu}_{4} \mathrm{NBArF}_{24} / \mathrm{CH}_{2} \mathrm{Cl}_{2}$. The $\mathrm{CV}$ of the solution was recorded. The $o$-chloranil solution was titrated with $20-40 \mu \mathrm{L}$ increments of 5, and a CV was recorded after each addition of $\mathbf{5}$. After the total concentration of 5 in the cell exceeded $2.5 \mathrm{mM}$, irreversibility was observed in the $\mathrm{CV}$ and formation of a precipitate was observed in the electrochemical cell. We attribute this irreversibility and precipitate formation to methylation of the quinone radical anion at large [5]. However, the desired equilibrium constants could still be estimated from the data that were 
obtained at low [5]. The experimental data in this range were reproduced by simulation of an EC mechanism.

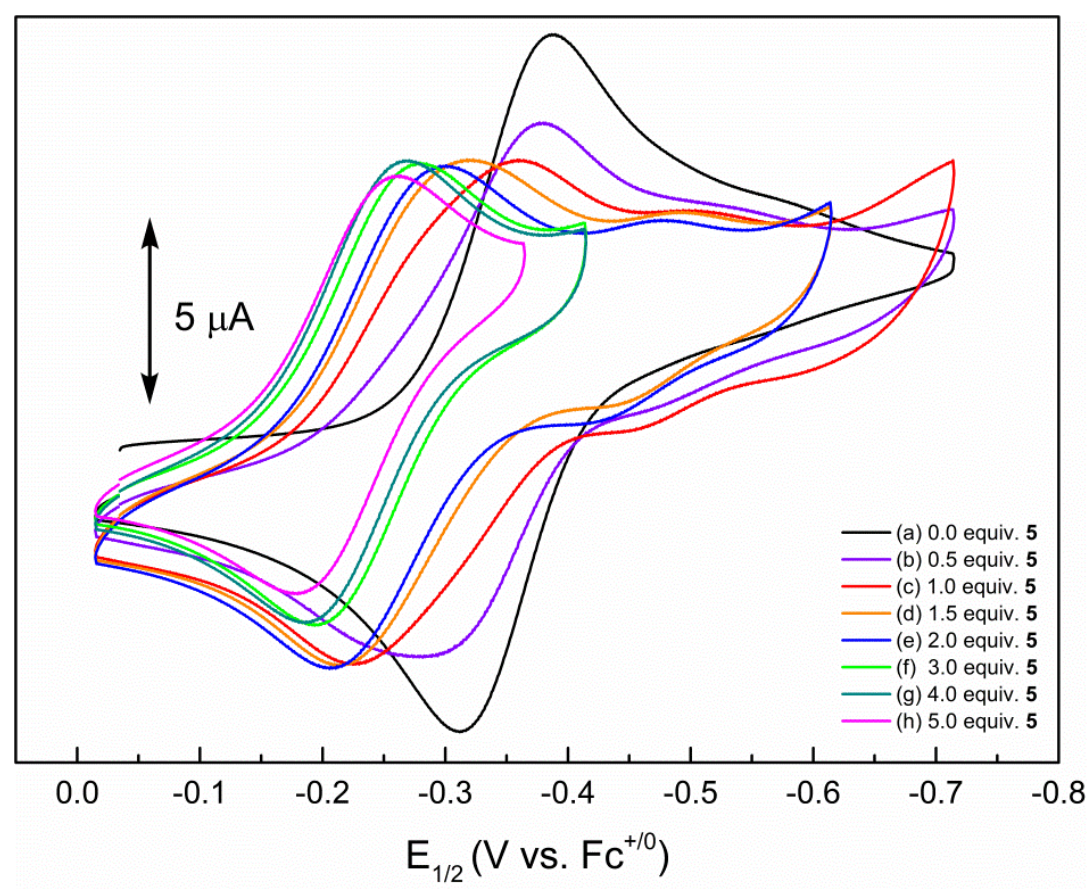

Figure 2.44. Full set of experimental $\mathrm{CVs}$ of $o$-chloranil (initial concentration $=0.5 \mathrm{mM}$ ) recorded in $0.1 \mathrm{M} \mathrm{nBu} \mathrm{Bu}_{4} \mathrm{NBArF}_{24} / \mathrm{CH}_{2} \mathrm{Cl}_{2}$ (glovebox) in the presence of increasing [5]. In scans (b)-(d), the additional current peaks at lower potential are attributed to the $2 \mathrm{e}^{-}$reduction. 


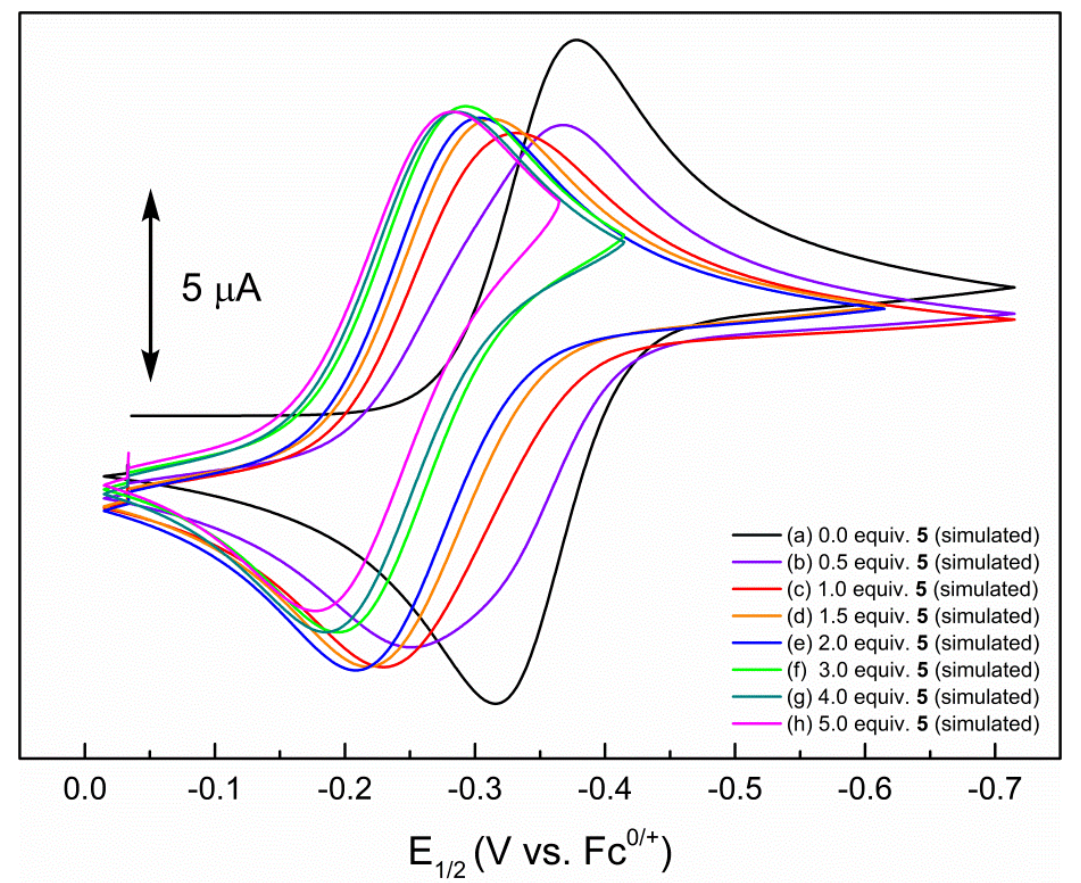

Figure 2.45. Full set of simulations of $\mathrm{CV}$ data for $o$-chloranil (initial concentration $=0.5 \mathrm{mM}$ ) recorded in $0.1 \mathrm{M} n \mathrm{Bu}_{4} \mathrm{NBArF}_{24} / \mathrm{CH}_{2} \mathrm{Cl}_{2}$ (glovebox) in the presence of increasing [5]

\section{CV experiments with 6}

A $0.1 \mathrm{M}$ stock solution of $6(100.6 \mathrm{mg})$ in $\mathrm{CH}_{2} \mathrm{Cl}_{2}$ was prepared in a $1 \mathrm{~mL}$ volumetric

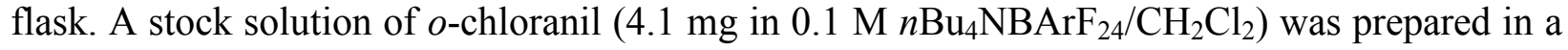
$1 \mathrm{~mL}$ volumetric flask. A $0.5 \mathrm{mM}$ solution of $o$-chloranil in $0.1 \mathrm{M} \mathrm{nBu} \mathrm{NBArF}_{24} / \mathrm{CH}_{2} \mathrm{Cl}_{2}$ was prepared in an electrochemical cell using $120 \mu \mathrm{L}$ of the $o$-chloranil stock solution and $3.9 \mathrm{~mL}$ of $0.1 \mathrm{M} n \mathrm{Bu}_{4} \mathrm{NBArF}_{24} / \mathrm{CH}_{2} \mathrm{Cl}_{2}$. The $\mathrm{CV}$ of the solution was recorded. The $o$-chloranil solution was titrated with 10-200 $\mu \mathrm{L}$ increments of $\mathbf{6}$, and a CV was recorded after each addition of $\mathbf{6}$. The experimental data were reproduced by simulation of a CEC mechanism. 


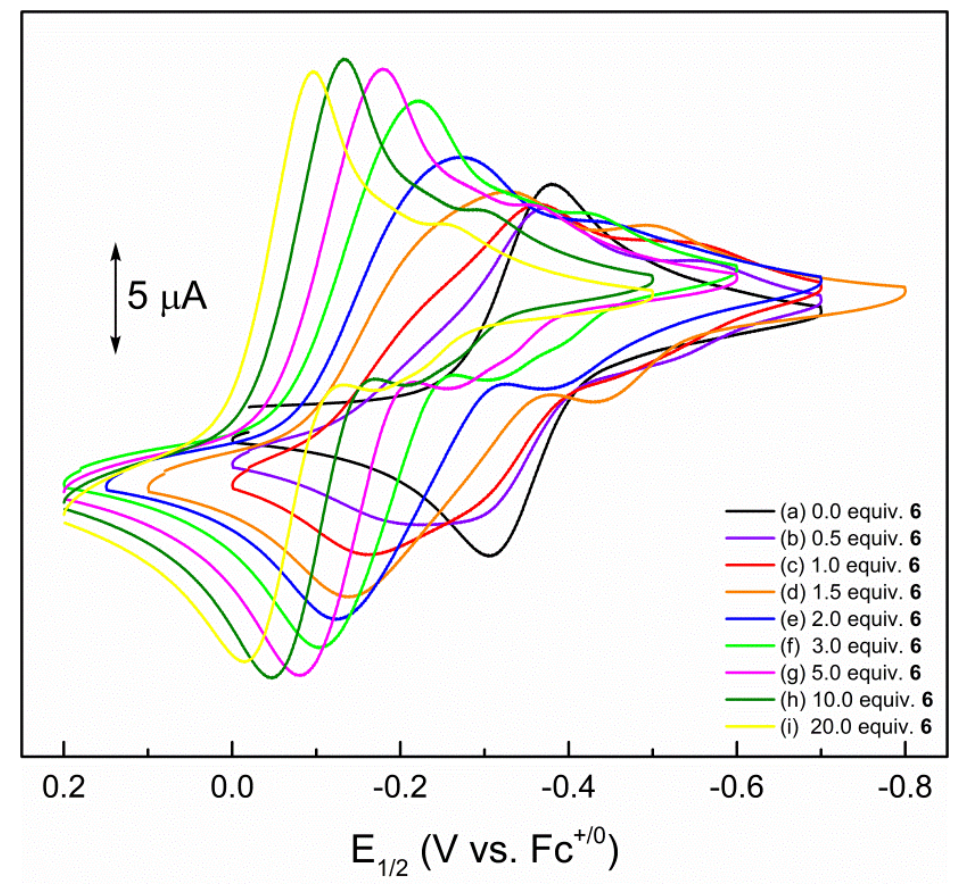

Figure 2.46. Full set of experimental $\mathrm{CVs}$ of $o$-chloranil (initial concentration $=0.5 \mathrm{mM}$ ) recorded in $0.1 \mathrm{M} \mathrm{nBu} \mathrm{BuArF}_{24} / \mathrm{CH}_{2} \mathrm{Cl}_{2}$ (glovebox) in the presence of increasing [6]. In scans (b)-(i), the additional current peaks at lower potential are attributed to the $2 \mathrm{e}^{-}$reduction.

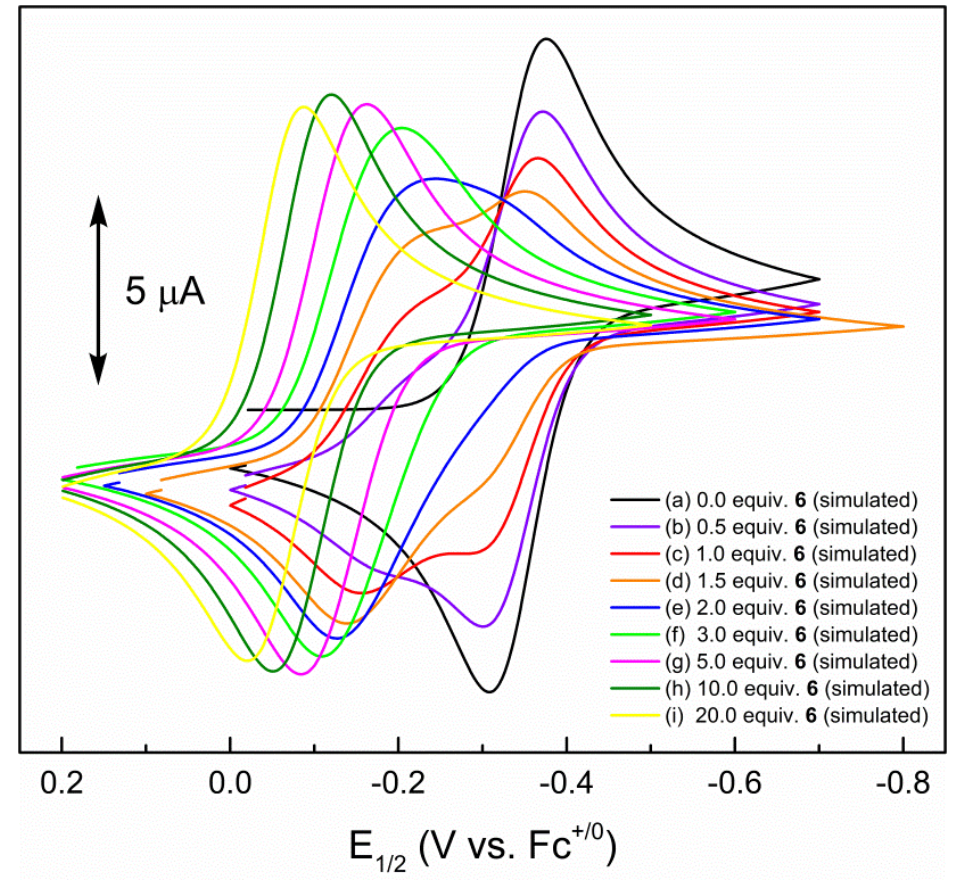

Figure 2.47. Full set of simulations of $\mathrm{CV}$ data for $o$-chloranil (initial concentration $=0.5 \mathrm{mM}$ ) recorded in $0.1 \mathrm{M} \mathrm{n} n \mathrm{Bu}_{4} \mathrm{NBArF}_{24} / \mathrm{CH}_{2} \mathrm{Cl}_{2}$ (glovebox) in the presence of increasing [6] 


\section{CV experiments with 7}

A $0.1 \mathrm{M}$ stock solution of $7(114.9 \mathrm{mg})$ in $\mathrm{CH}_{2} \mathrm{Cl}_{2}$ was prepared in a $1 \mathrm{~mL}$ volumetric flask. A stock solution of $o$-chloranil $\left(8.6 \mathrm{mg}\right.$ in $\left.0.1 \mathrm{M} \mathrm{nBu}{ }_{4} \mathrm{NBArF}_{24} / \mathrm{CH}_{2} \mathrm{Cl}_{2}\right)$ was prepared in a $1 \mathrm{~mL}$ volumetric flask. A $0.5 \mathrm{mM}$ solution of $o$-chloranil in $0.1 \mathrm{M} n \mathrm{Bu}_{4} \mathrm{NBArF}_{24} / \mathrm{CH}_{2} \mathrm{Cl}_{2}$ was prepared in an electrochemical cell using $57 \mu \mathrm{L}$ of the $o$-chloranil stock solution and $3.95 \mathrm{~mL}$ of $0.1 \mathrm{M} n \mathrm{Bu}_{4} \mathrm{NBArF}_{24} / \mathrm{CH}_{2} \mathrm{Cl}_{2}$. The $\mathrm{CV}$ of the solution was recorded. The $o$-chloranil solution was titrated with 10-200 $\mu \mathrm{L}$ increments of 7 , and a CV was recorded after each addition of 7 . The experimental data were reproduced by simulation of a CEC mechanism.

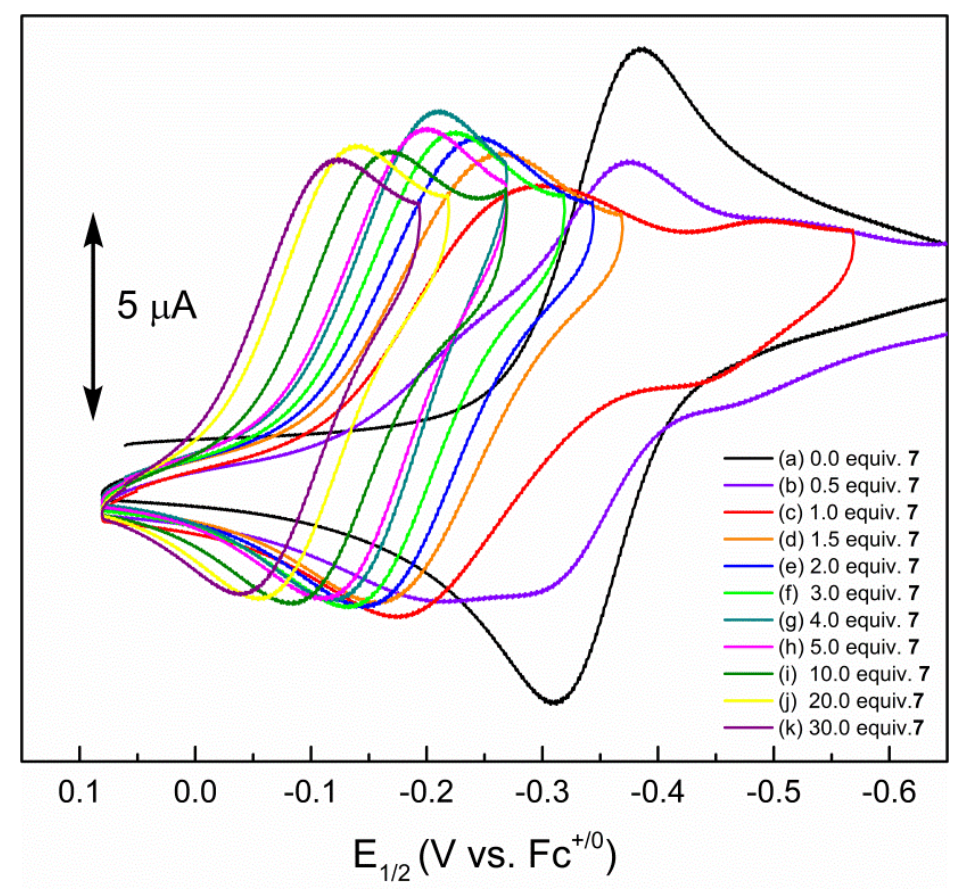

Figure 2.48. Full set of experimental $\mathrm{CVs}$ of $o$-chloranil (initial concentration $=0.5 \mathrm{mM}$ ) recorded in $0.1 \mathrm{M} \mathrm{nBu} \mathrm{Bu}_{4} \mathrm{NBArF}_{24} / \mathrm{CH}_{2} \mathrm{Cl}_{2}$ (glovebox) in the presence of increasing [7]. In scans (b)-(c), the additional current peaks at lower potential are attributed to the $2 \mathrm{e}^{-}$reduction. 


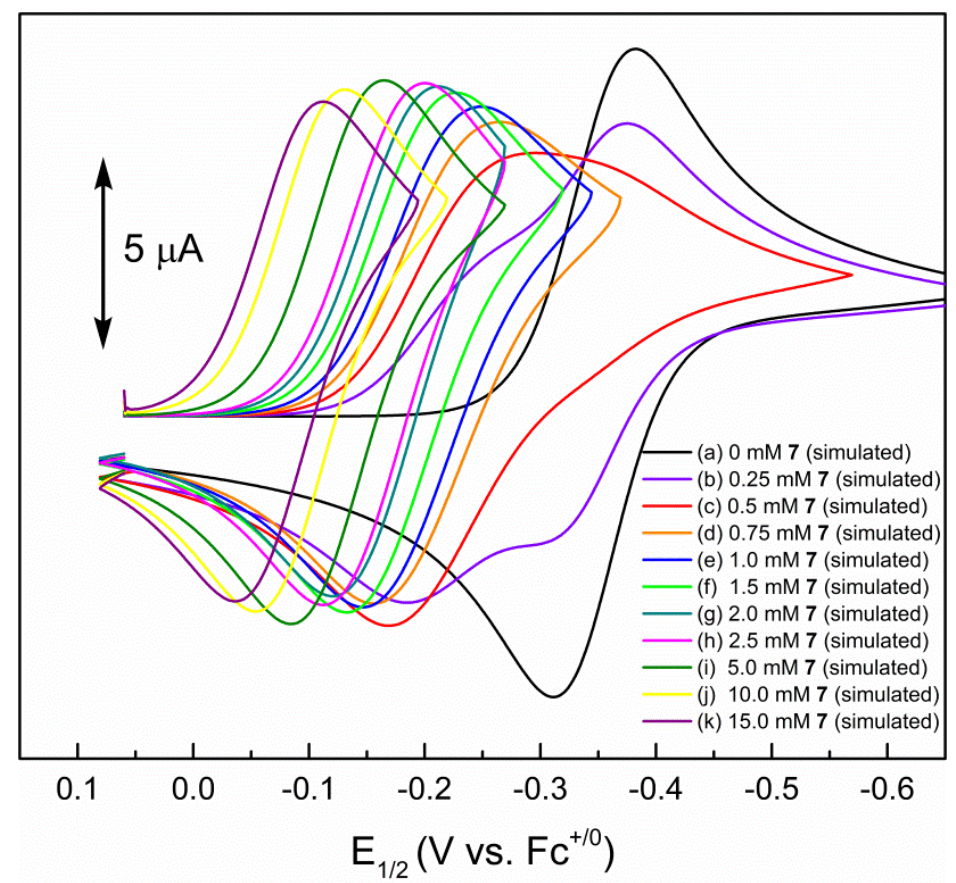

Figure 2.49. Full set of simulations of $\mathrm{CV}$ data for $o$-chloranil (initial concentration $=0.5 \mathrm{mM}$ ) recorded in $0.1 \mathrm{M} n \mathrm{Bu}_{4} \mathrm{NBArF}_{24} / \mathrm{CH}_{2} \mathrm{Cl}_{2}$ (glovebox) in the presence of increasing [7]

\section{CV experiments with 8}

A $0.1 \mathrm{M}$ stock solution of $8(108.1 \mathrm{mg})$ in $\mathrm{CH}_{2} \mathrm{Cl}_{2}$ was prepared in a $1 \mathrm{~mL}$ volumetric flask. A stock solution of $o$-chloranil $\left(4.1 \mathrm{mg}\right.$ in $0.1 \mathrm{M} n \mathrm{Bu}_{4} \mathrm{NBArF}_{24} / \mathrm{CH}_{2} \mathrm{Cl}_{2}$ ) was prepared in a $1 \mathrm{~mL}$ volumetric flask. A $0.5 \mathrm{mM}$ solution of $o$-chloranil in $0.1 \mathrm{M} \mathrm{nBu} \mathrm{NBArF}_{24} / \mathrm{CH}_{2} \mathrm{Cl}_{2}$ was prepared in an electrochemical cell using $120 \mu \mathrm{L}$ of the $o$-chloranil stock solution and $3.9 \mathrm{~mL}$ of 0.1 $\mathrm{M} n \mathrm{Bu}_{4} \mathrm{NBArF}_{24} / \mathrm{CH}_{2} \mathrm{Cl}_{2}$. The $\mathrm{CV}$ of the solution was recorded. The $o$-chloranil solution was titrated with $10-200 \mu \mathrm{L}$ increments of $\mathbf{8}$, and a CV was recorded after each addition of $\mathbf{8}$. The experimental data were reproduced by simulation of a CEC mechanism. 


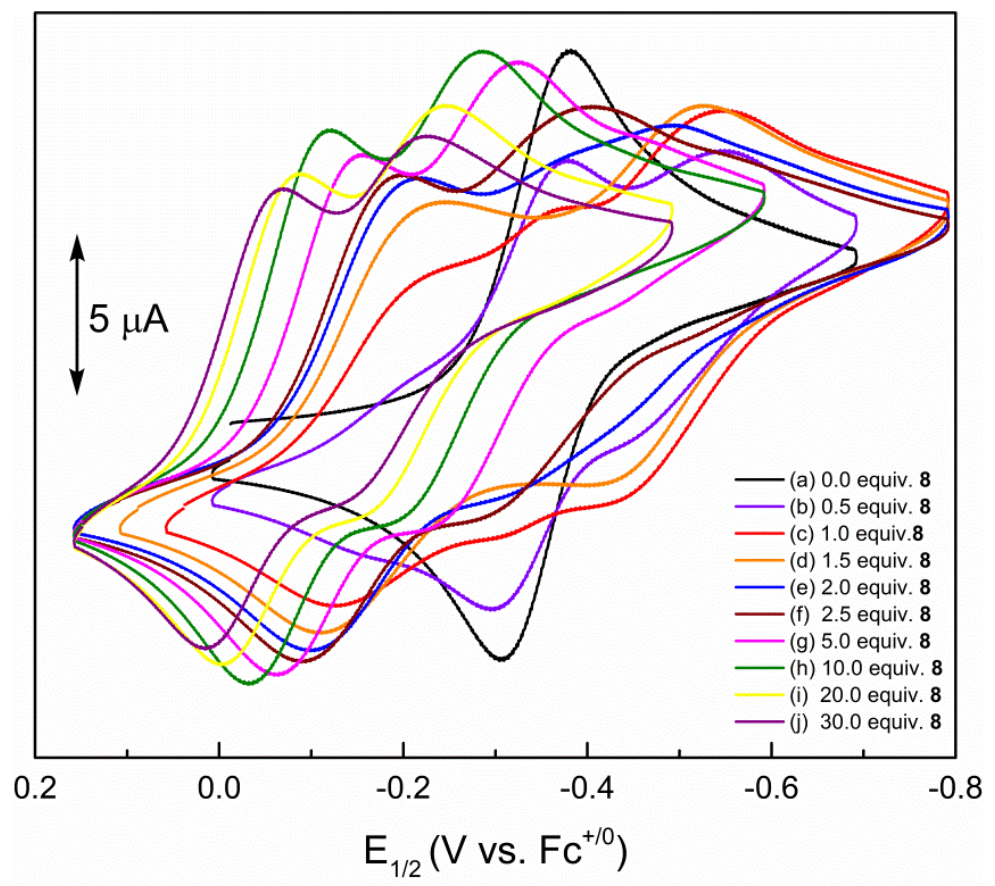

Figure 2.50. Full set of experimental $\mathrm{CVs}$ of $o$-chloranil (initial concentration $=0.5 \mathrm{mM}$ ) recorded in $0.1 \mathrm{M} \mathrm{nBu} \mathrm{BuArF}_{24} / \mathrm{CH}_{2} \mathrm{Cl}_{2}$ (glovebox) in the presence of increasing [8]. In scans (b)-(j), the additional current peaks at lower potential are attributed to the $2 \mathrm{e}^{-}$reduction.

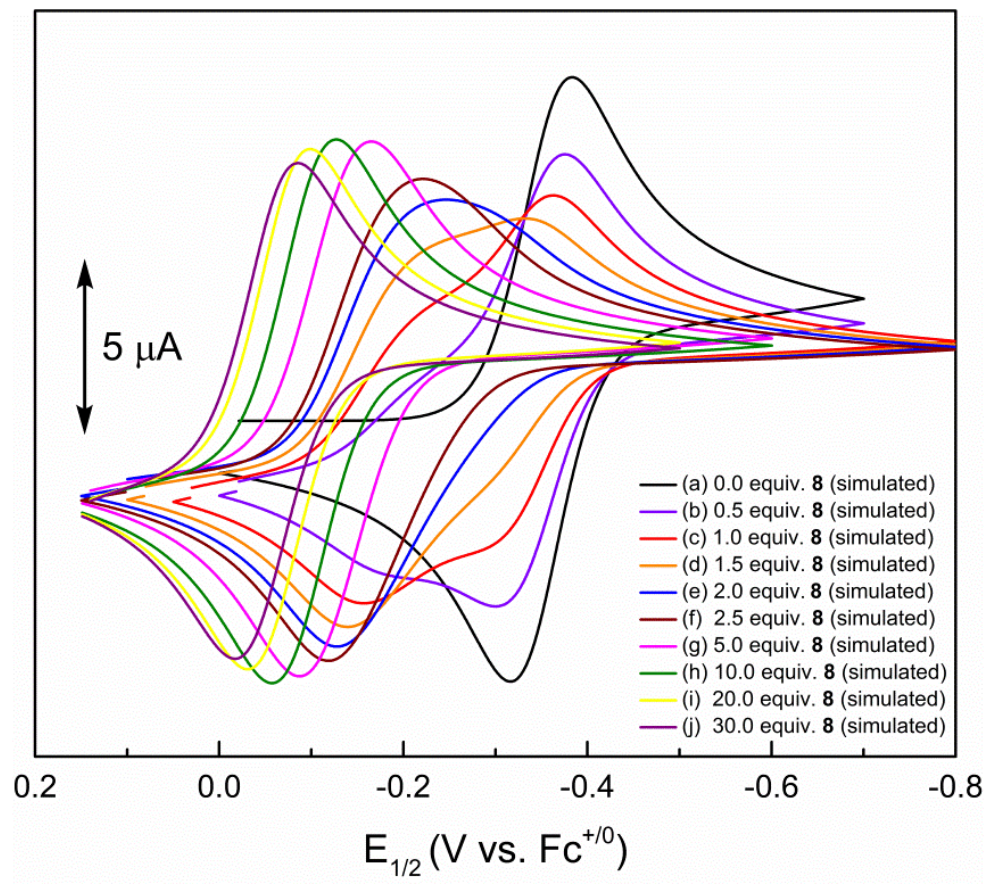

Figure 2.51. Full set of simulations of $\mathrm{CV}$ data for $o$-chloranil (initial concentration $=0.5 \mathrm{mM}$ ) recorded in $0.1 \mathrm{M} n \mathrm{Bu}_{4} \mathrm{NBArF}_{24} / \mathrm{CH}_{2} \mathrm{Cl}_{2}$ (glovebox) in the presence of increasing [8] 


\section{CV experiments with 9}

A $0.1 \mathrm{M}$ stock solution of $9(107.0 \mathrm{mg})$ in $\mathrm{CH}_{2} \mathrm{Cl}_{2}$ was prepared in a $1 \mathrm{~mL}$ volumetric flask. A stock solution of $o$-chloranil $\left(7.3 \mathrm{mg}\right.$ in $\left.0.1 \mathrm{M} n \mathrm{Bu}_{4} \mathrm{NBArF}_{24} / \mathrm{CH}_{2} \mathrm{Cl}_{2}\right)$ was prepared in a $2 \mathrm{~mL}$ volumetric flask. A $0.5 \mathrm{mM}$ solution of $o$-chloranil in $0.1 \mathrm{M} n \mathrm{Bu}_{4} \mathrm{NBArF}_{24} / \mathrm{CH}_{2} \mathrm{Cl}_{2}$ was prepared in an electrochemical cell using $135 \mu \mathrm{L}$ of the $o$-chloranil stock solution and $3.85 \mathrm{~mL}$ of $0.1 \mathrm{M} n \mathrm{Bu}_{4} \mathrm{NBArF}_{24} / \mathrm{CH}_{2} \mathrm{Cl}_{2}$. The $\mathrm{CV}$ of the solution was recorded. The $o$-chloranil solution was titrated with 10-200 $\mu \mathrm{L}$ increments of 9, and a CV was recorded after each addition of 9. The experimental data were somewhat reproduced by simulation of a CEC mechanism.

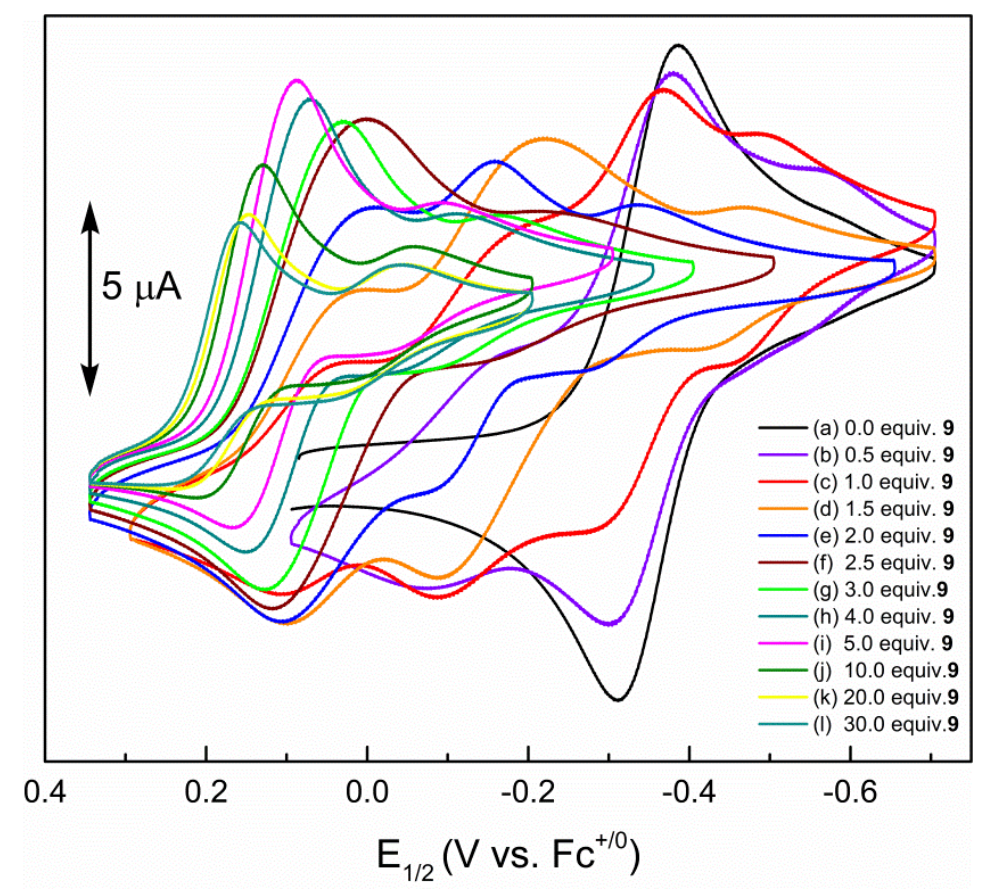

Figure 2.52. Full set of experimental CVs of $0.5 \mathrm{mM} o$-chloranil recorded in $0.1 \mathrm{M}$ $n \mathrm{Bu}_{4} \mathrm{NBArF}_{24} / \mathrm{CH}_{2} \mathrm{Cl}_{2}$ (glovebox) in the presence of increasing [9]. In scans (b)-(1), the additional current peaks at lower potential are attributed to the $2 \mathrm{e}^{-}$reduction. 


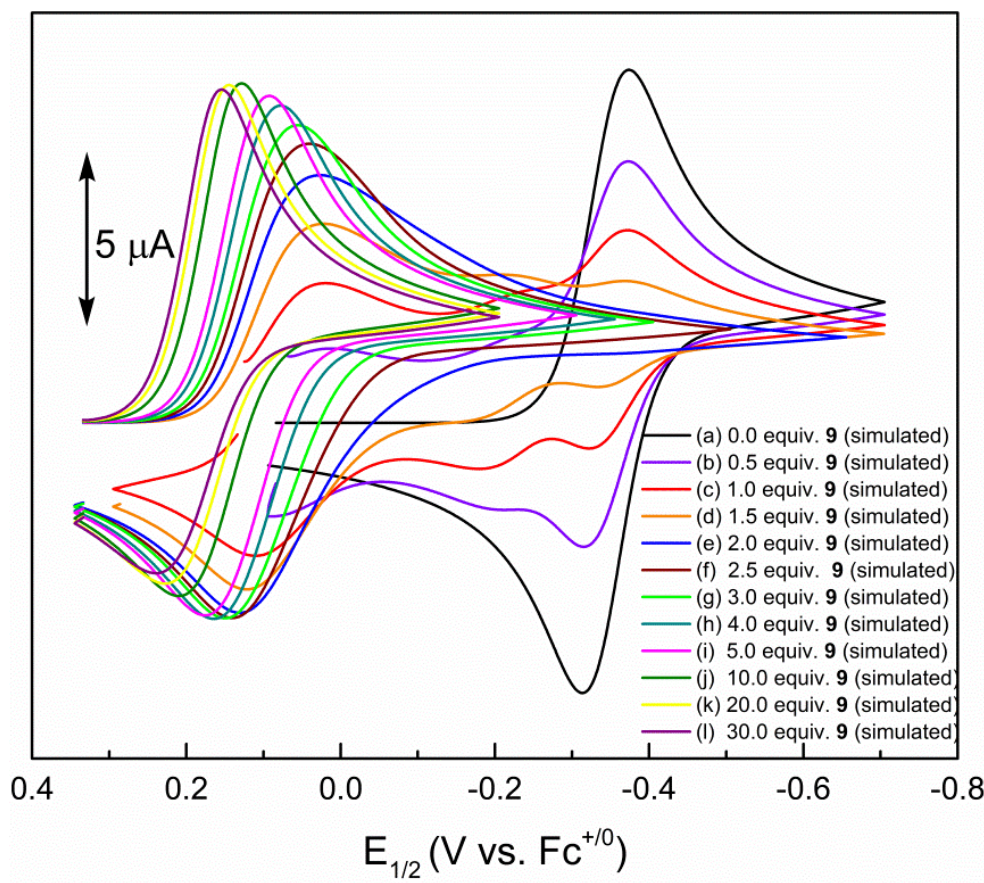

Figure 2.53. Full set of simulations of $\mathrm{CV}$ data for $o$-chloranil (initial concentration $=0.5 \mathrm{mM}$ ) recorded in $0.1 \mathrm{M} n \mathrm{Bu}_{4} \mathrm{NBArF}_{24} / \mathrm{CH}_{2} \mathrm{Cl}_{2}$ (glovebox) in the presence of increasing [9]. The irreversibility that develops over the course of the experimental titration is somewhat replicated in this simulation.

\section{2a.7.4 Measurement of binding constants for neutral $Q$}

\section{Benesi-Hildebrand plot for $1 \cdot Q$ binding}
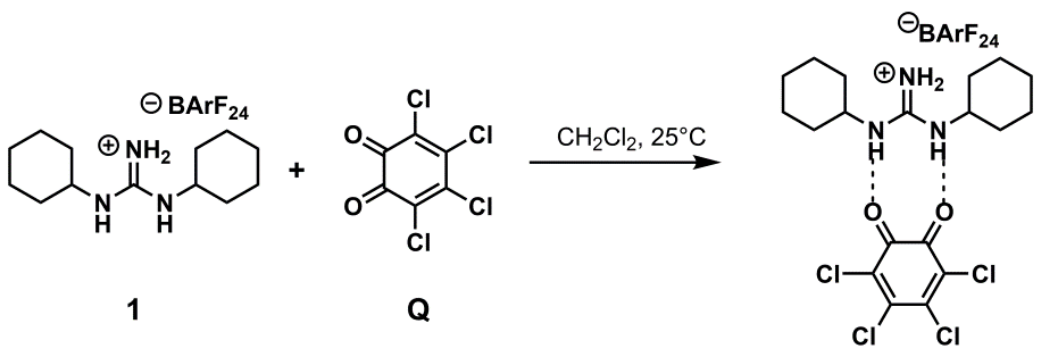

A stock solution of $o$-chloranil $(19 \mathrm{mM})$ was prepared in $\mathrm{CH}_{2} \mathrm{Cl}_{2}$ in a $2 \mathrm{~mL}$ volumetric flask. A stock solution of $1(19 \mathrm{mM})$ was prepared in $\mathrm{CH}_{2} \mathrm{Cl}_{2}$ in a $1 \mathrm{~mL}$ volumetric flask. A quartz cuvette was charged with $1.5 \mathrm{~mL} \mathrm{CH}_{2} \mathrm{Cl}_{2}$, followed by $8 \mu \mathrm{L} o$-chloranil (total sample concentration $=0.1 \mathrm{mM})$. The cuvette was titrated with increments of $\mathbf{1}$. 


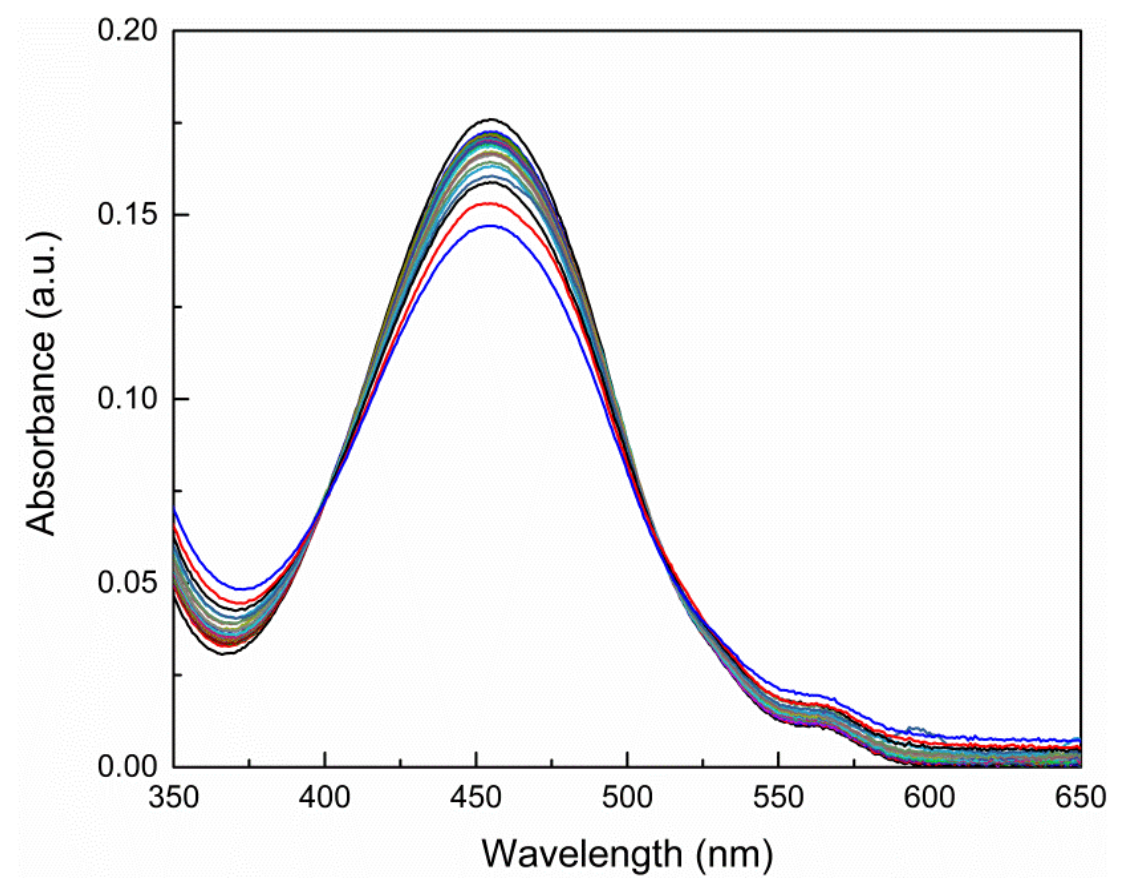

Figure 2.54. Absorbance vs. wavelength data for titration of $o$-chloranil with increments of $\mathbf{1}$.

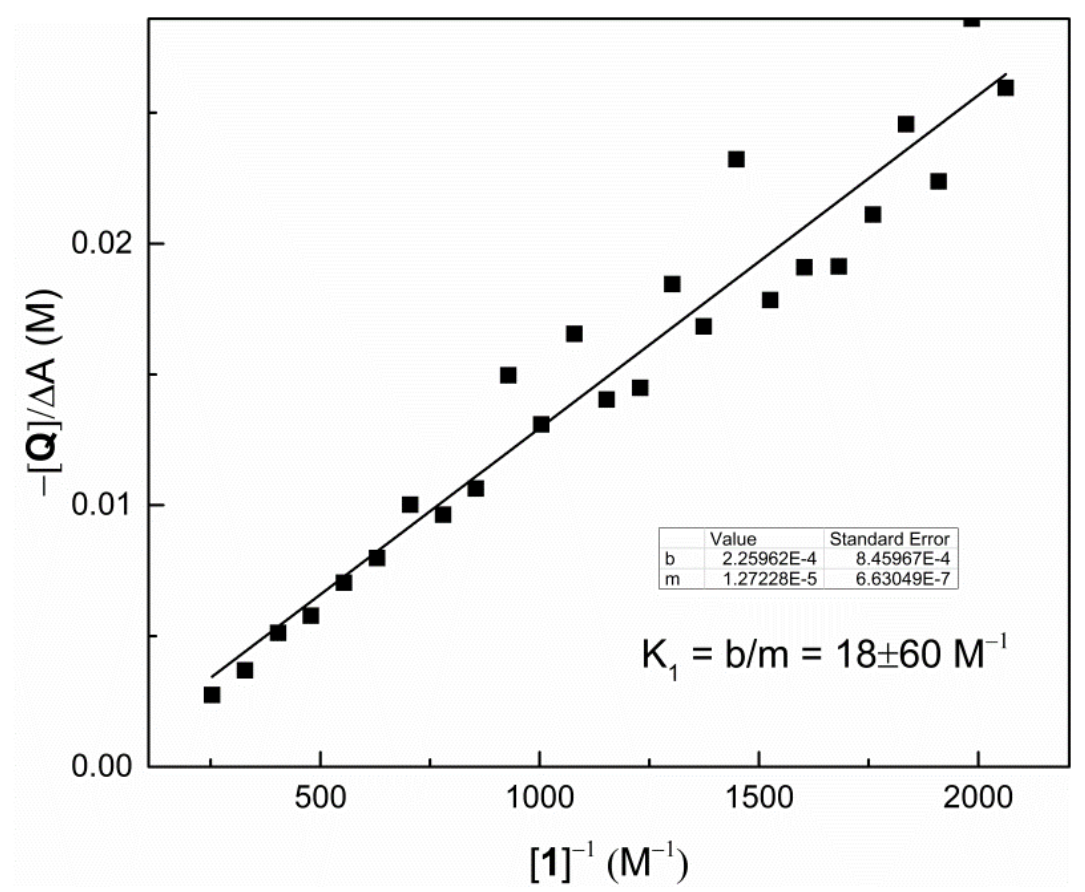

Figure 2.55. Benesi-Hildebrand plot for binding of 1 and $o$-chloranil determined using absorbance at $454 \mathrm{~nm}$. 


\section{Benesi-Hildebrand plot for $2 \cdot \mathrm{Q}$ binding}
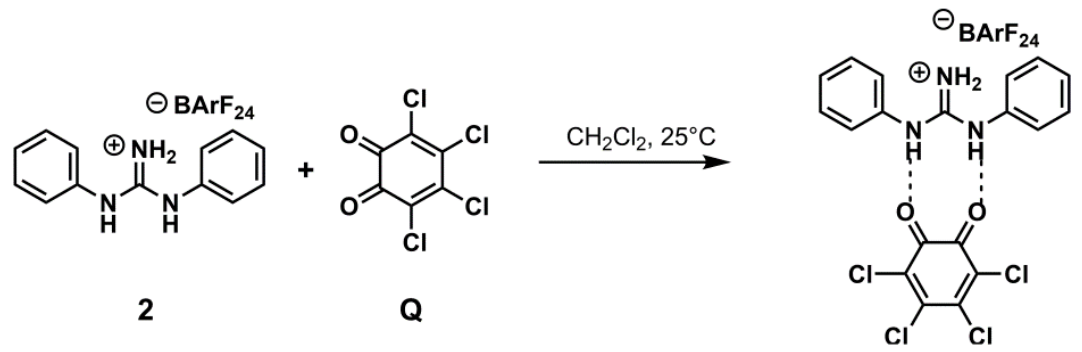

A stock solution of $o$-chloranil $(19 \mathrm{mM})$ was prepared in $\mathrm{CH}_{2} \mathrm{Cl}_{2}$ in a $2 \mathrm{~mL}$ volumetric flask. A stock solution of $2(23 \mathrm{mM})$ was prepared in $\mathrm{CH}_{2} \mathrm{Cl}_{2}$ in a $1 \mathrm{~mL}$ volumetric flask. A quartz cuvette was charged with $1.5 \mathrm{~mL} \mathrm{CH}_{2} \mathrm{Cl}_{2}$, followed by $8 \mu \mathrm{L} o$-chloranil (total sample concentration $=0.1 \mathrm{mM})$. The cuvette was titrated with increments of 2 .

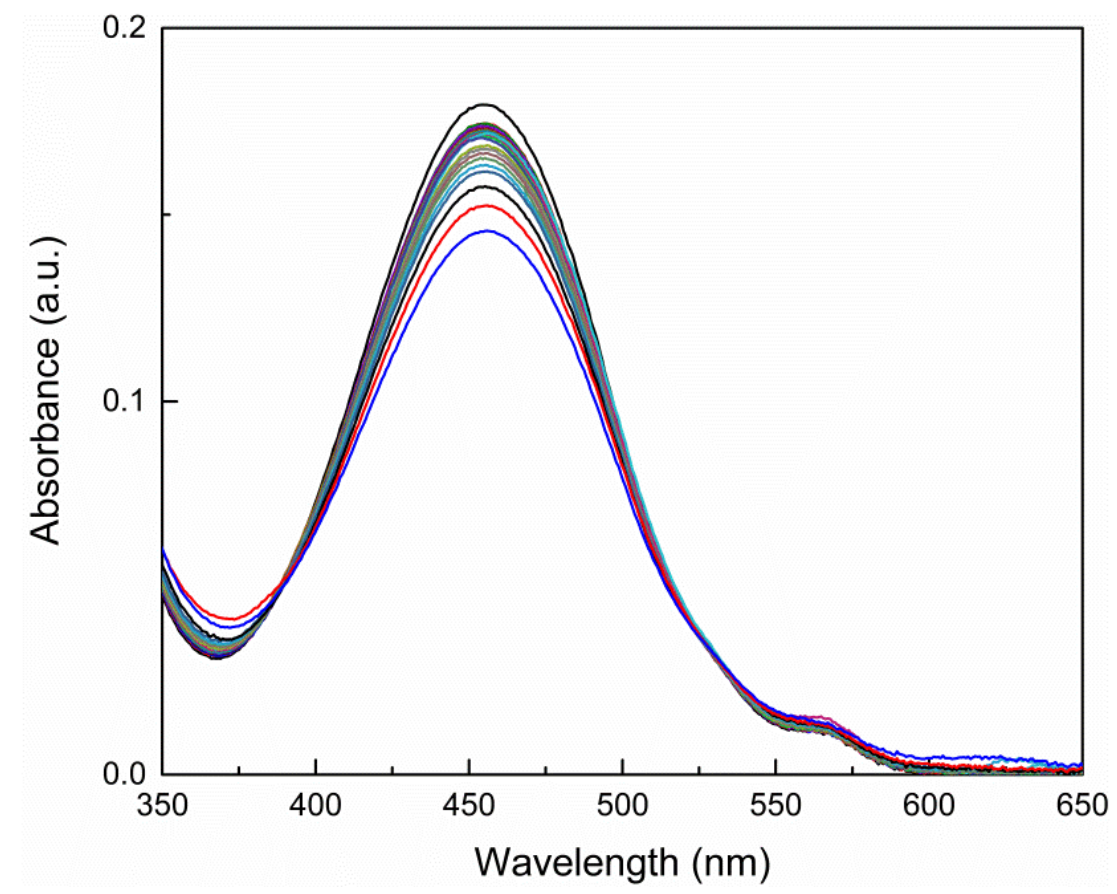

Figure 2.56. Absorbance vs. wavelength data for titration of $o$-chloranil with increments of $\mathbf{2}$. 


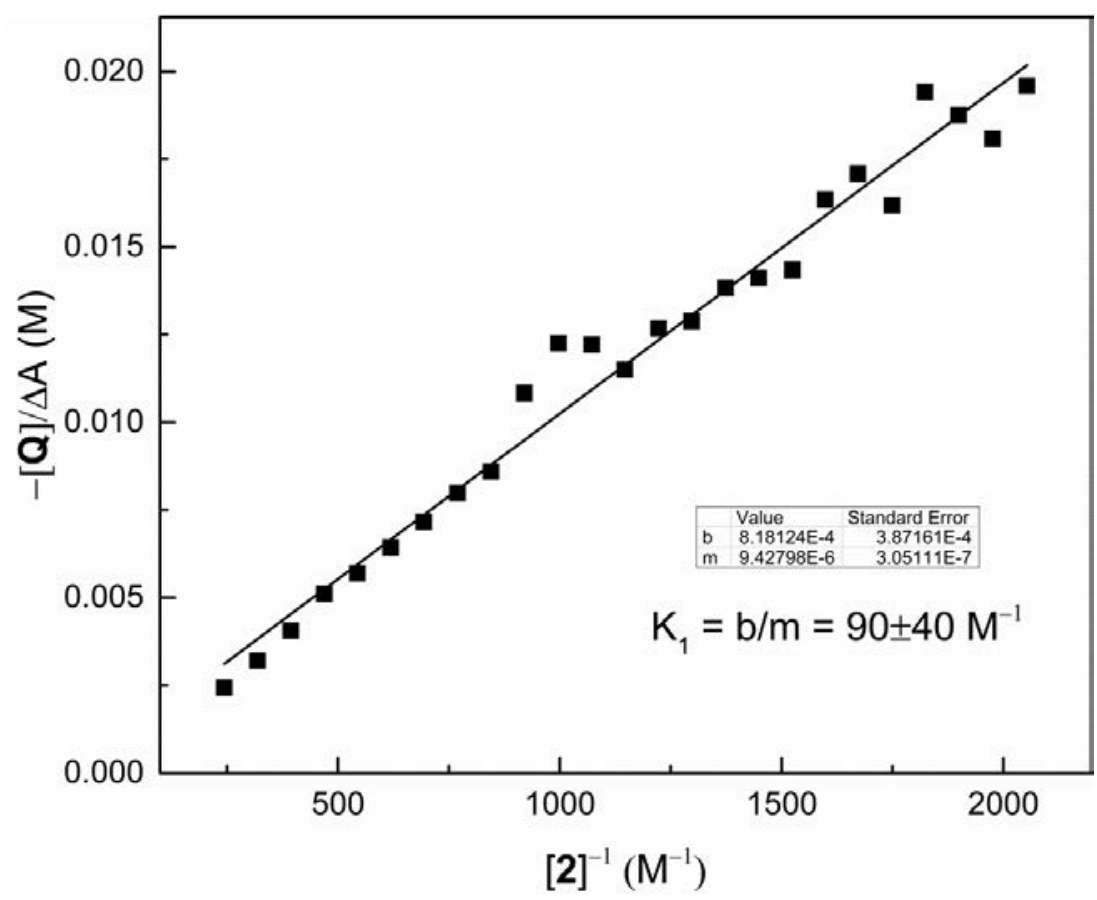

Figure 2.57. Benesi-Hildebrand plot for binding of $\mathbf{2}$ and $o$-chloranil determined using absorbance at $454 \mathrm{~nm}$.

\section{Benesi-Hildebrand plot for $3 \cdot Q$ binding}<smiles>CCCOC(=O)c1cc(NC(=O)Nc2cc(C(F)(F)F)cc(C(F)(F)F)c2)cc(C(=O)OCC)c1</smiles>

3

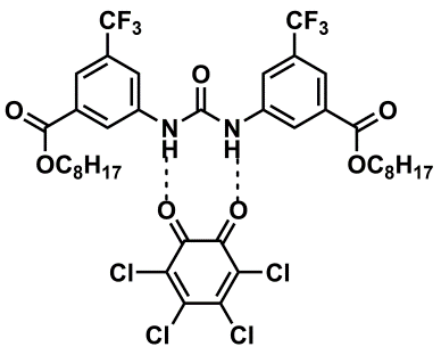

A stock solution of $o$-chloranil $(19 \mathrm{mM})$ was prepared in $\mathrm{CH}_{2} \mathrm{Cl}_{2}$ in a $2 \mathrm{~mL}$ volumetric flask. A stock solution of $3(34 \mathrm{mM})$ was prepared in $\mathrm{CH}_{2} \mathrm{Cl}_{2}$ with $2 \%$ TBME in a $1 \mathrm{~mL}$ volumetric flask. A quartz cuvette was charged with $1.5 \mathrm{~mL} \mathrm{CH}_{2} \mathrm{Cl}_{2}$, followed by $8 \mu \mathrm{L} o$ chloranil (total sample concentration $=0.1 \mathrm{mM}$ ). The cuvette was titrated with increments of 3 . 


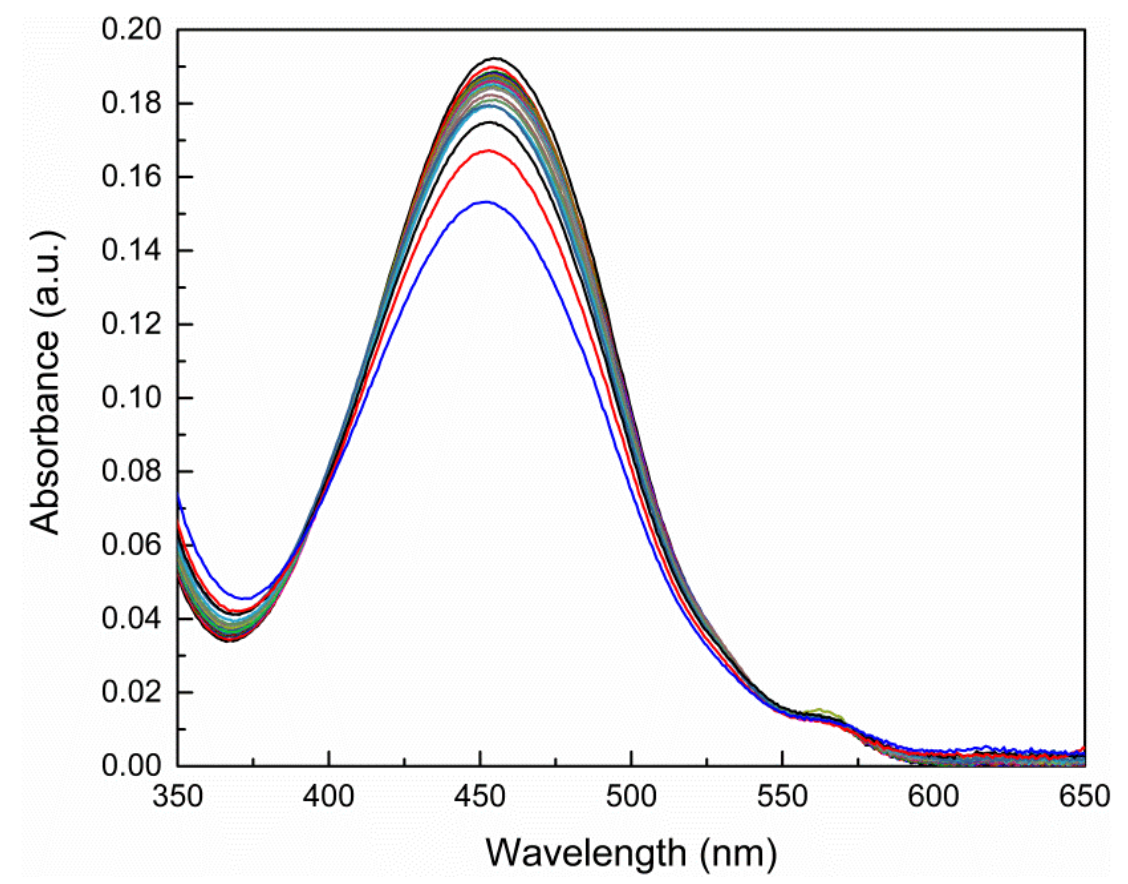

Figure 2.58. Absorbance vs. wavelength data for titration of $o$-chloranil with increments of $\mathbf{3}$.

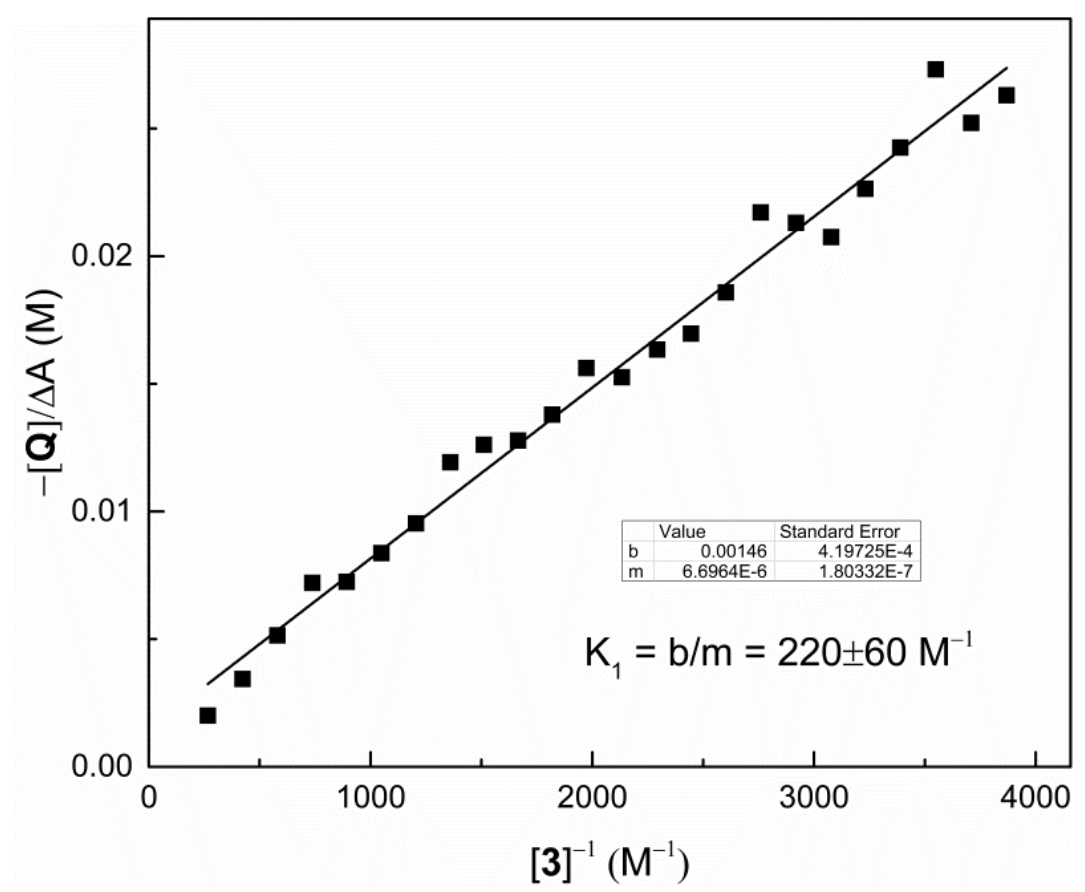

Figure 2.59. Benesi-Hildebrand plot for binding of $\mathbf{3}$ and $o$-chloranil determined using absorbance at $454 \mathrm{~nm}$. 


\section{Benesi-Hildebrand plot for $4 \cdot \mathrm{Q}$ binding}

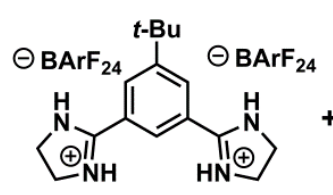

4

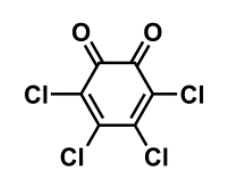

Q
$\stackrel{\mathrm{CH}_{2} \mathrm{Cl}_{2}, 25^{\circ} \mathrm{C}}{\longrightarrow}$

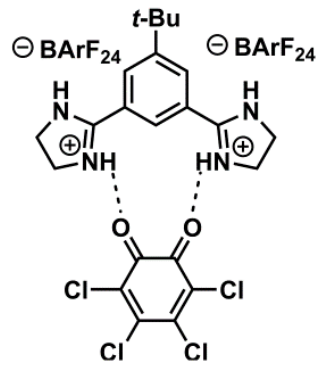

A stock solution of $o$-chloranil $(18 \mathrm{mM})$ was prepared in $\mathrm{CH}_{2} \mathrm{Cl}_{2}$ in a $2 \mathrm{~mL}$ volumetric flask. A stock solution of $4(16 \mathrm{mM})$ was prepared in $\mathrm{CH}_{2} \mathrm{Cl}_{2}$ in a $1 \mathrm{~mL}$ volumetric flask. A quartz cuvette was charged with $1.5 \mathrm{~mL} \mathrm{CH}_{2} \mathrm{Cl}_{2}$, followed by $8 \mu \mathrm{L}$ o-chloranil (total sample concentration $=0.1 \mathrm{mM})$. The cuvette was titrated with increments of 4 .

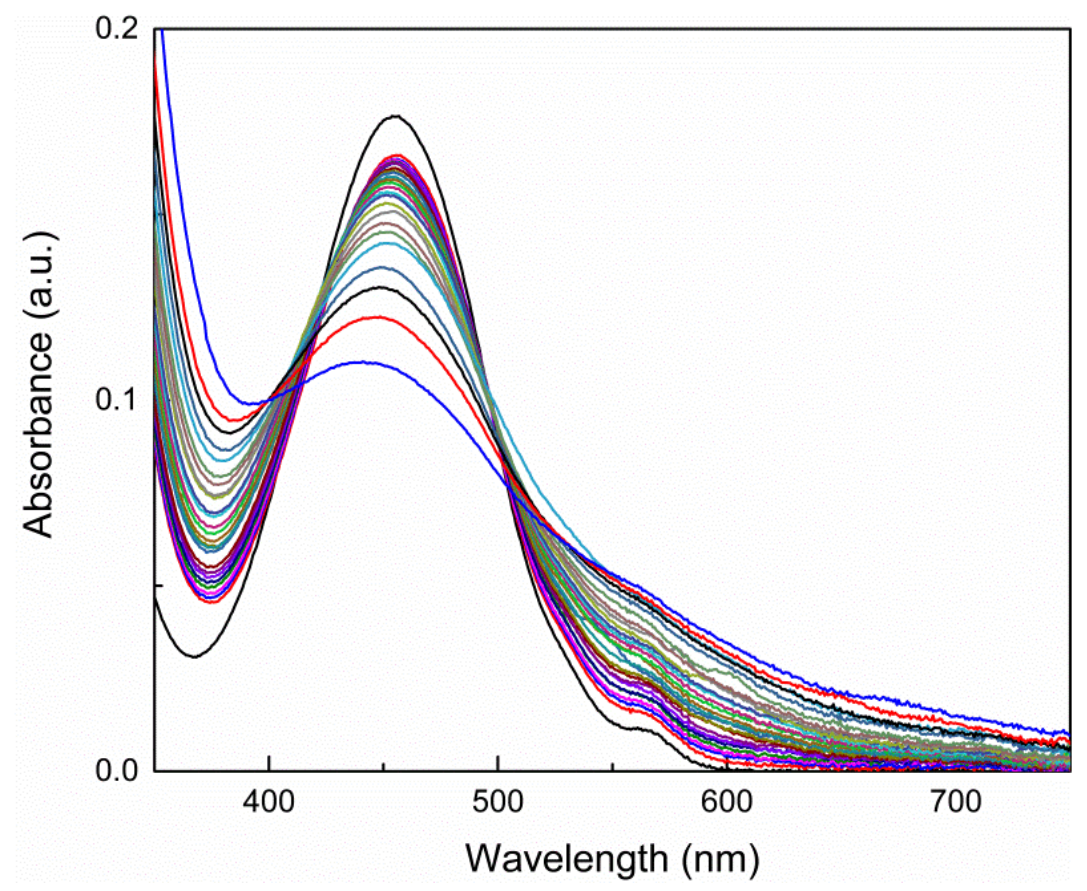

Figure 2.60. Absorbance vs. wavelength data for titration of $o$-chloranil with increments of 4 . 


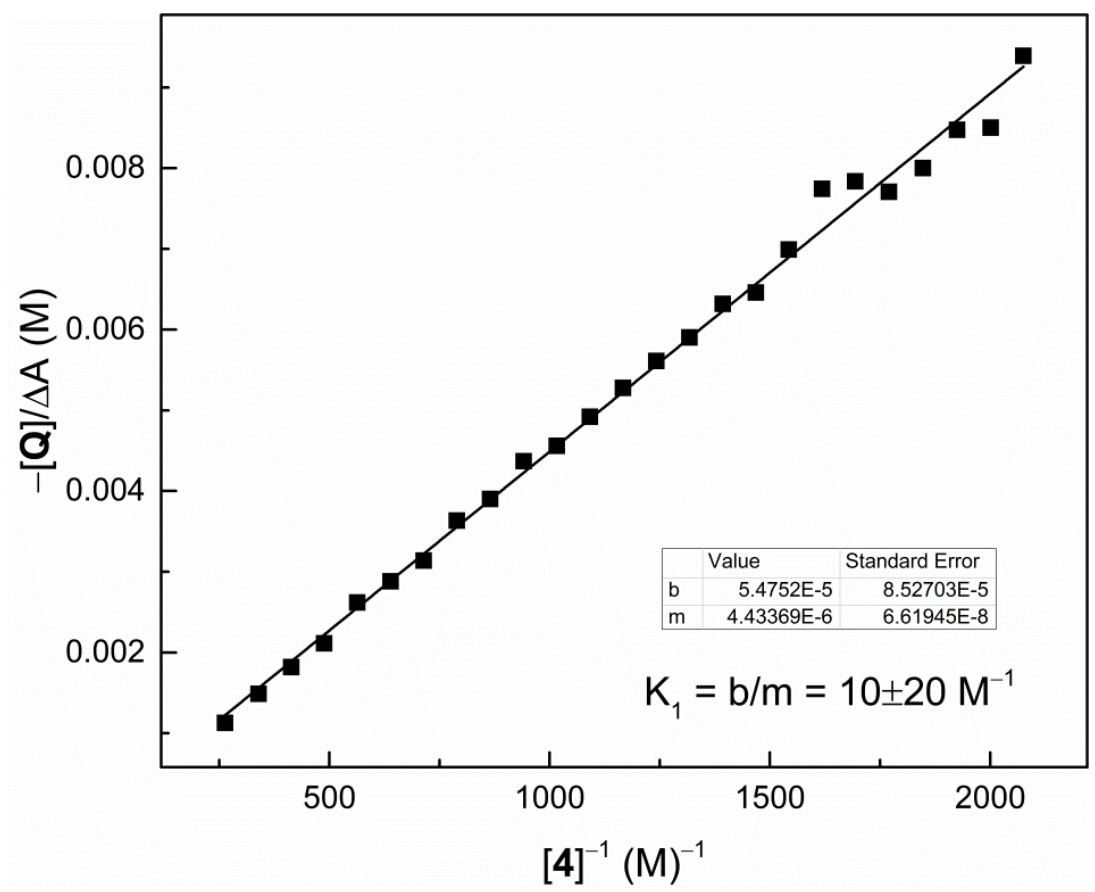

Figure 2.61. Benesi-Hildebrand plot for binding of 4 and $o$-chloranil determined using absorbance at $454 \mathrm{~nm}$.

\section{2a.7.5 Geometry optimizations for $(\mathrm{HBD})_{\mathrm{n}} \cdot \mathrm{Q}^{\circ-}$ complexes}

Calculations were executed on the Harvard University Odyssey computing cluster using the Gaussian $09^{26}$ program with the B3LYP method ${ }^{27}$ and the M06-2X method, ${ }^{28}$ using the 6-

\footnotetext{
${ }^{26}$ Gaussian 09, Revision A.02, Frisch, M. J.; Trucks, G. W.; Schlegel, H. B.; Scuseria, G. E.; Robb, M. A.; Cheeseman, J. R.; Scalmani, G.; Barone, V.; Mennucci, B.; Petersson, G. A.; Nakatsuji, H.; Caricato, M.; Li, X.; Hratchian, H. P.; Izmaylov, A. F.; Bloino, J.; Zheng, G.; Sonnenberg, J. L.; Hada, M.; Ehara, M.; Toyota, K.; Fukuda, R.; Hasegawa, J.; Ishida, M.; Nakajima, T.; Honda, Y.; Kitao, O.; Nakai, H.; Vreven, T.; Montgomery, Jr., J. A.; Peralta, J. E.; Ogliaro, F.; Bearpark, M.; Heyd, J. J.; Brothers, E.; Kudin, K. N.; Staroverov, V. N.; Kobayashi, R.; Normand, J.; Raghavachari, K.; Rendell, A.; Burant, J. C.; Iyengar, S. S.; Tomasi, J.; Cossi, M.; Rega, N.; Millam, J. M.; Klene, M.; Knox, J. E.; Cross, J. B.; Bakken, V.; Adamo, C.; Jaramillo, J.; Gomperts, R.; Stratmann, R. E.; Yazyev, O.; Austin, A. J.; Cammi, R.; Pomelli, C.; Ochterski, J. W.; Martin, R. L.; Morokuma, K.; Zakrzewski, V. G.; Voth, G. A.; Salvador, P.; Dannenberg, J. J.; Dapprich, S.; Daniels, A. D.; Farkas, O.; Foresman, J. B.; Ortiz, J. V.; Cioslowski, J.; Fox, D. J. Gaussian, Inc., Wallingford CT, 2009.
}

${ }^{27}$ (a) Becke, A. D. J. Chem. Phys. 1993, 98, 1372-1377. (b) Lee, C; Yang, W.; Parr, R. G. Phys. Rev. B 1988, 37, 785-789.

${ }^{28}$ Zhao, Y.; Truhlar, D. G. Theor. Chem. Acc. 2008, 120, 215-241. 
$31 \mathrm{G}(\mathrm{d}, \mathrm{p})$ basis set. ${ }^{29}$ All energies are counterpoise-corrected, ${ }^{30}$ dispersion-corrected (for B3LYP calculations), ${ }^{31}$ and solvent-corrected using implicit solvation (PCM). ${ }^{32}$

$\left(N, N^{\prime} \text {-diisopropylguanidinium }\right)_{2} \cdot \mathbf{Q}^{\bullet-}$ complex

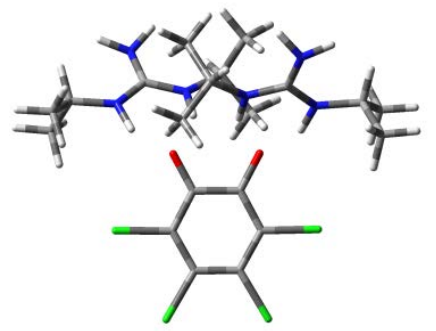

E(UB3LYP): -3103.69707671

Counterpoise correction: 0.00419822

Dispersion correction: -0.15062399

PCM correction: 0.1372927

Cartesian coordinates:

$\begin{array}{lrrrrrrr}\mathrm{C} & 0.661545 & 3.522671 & 0.217488 & \mathrm{H} & -5.86571 & -1.51055 & -1.96302 \\ \mathrm{C} & -0.66234 & 3.52236 & -0.21767 & \mathrm{H} & -4.44856 & -0.44161 & -1.86639 \\ \mathrm{C} & -1.31826 & 2.289386 & -0.43231 & \mathrm{C} & -5.60987 & -0.76239 & 0.688427 \\ \mathrm{C} & -0.69348 & 1.044965 & -0.22644 & \mathrm{H} & -6.66068 & -0.85918 & 0.390774 \\ \mathrm{C} & 0.693772 & 1.045283 & 0.226697 & \mathrm{H} & -5.53651 & -0.98063 & 1.759504 \\ \mathrm{C} & 1.318017 & 2.290036 & 0.432321 & \mathrm{H} & -5.29354 & 0.273055 & 0.525748 \\ \mathrm{O} & 1.275158 & -0.10654 & 0.426478 & \mathrm{~N} & 3.324976 & -1.62554 & -0.27435 \\ \mathrm{O} & -1.27437 & -0.10721 & -0.42602 & \mathrm{C} & 2.742763 & -2.26764 & -1.29572\end{array}$

${ }^{29}$ (a) Ditchfield, R.; Hehre, W. J.; Pople, J. A. J. Chem. Phys. 1971, 54, 724-728. (b) Hehre, W. J.; Ditchfield, R.; Pople, J. A. J. Chem. Phys. 1972, 56, 2257-2261. (c) Hariharan, P. C.; Pople, J. A. Theor. Chim. Acta. 1973, 28, 213-222.

${ }^{30}$ Boys, S. F.; Bernardi, F. Mol. Phys. 1970, 19, 553-566.

${ }^{31}$ Grimme, S.; Antony, J.; Ehrlich, S.; Krieg, H. J. Chem. Phys. 2010, 132, 154104

${ }^{32}$ (a) Miertuš, S.; Scrocco, E.; Tomasi, J. Chem. Phys. 1981, 55, 117-129. (b) Pascual-Ahuir, J. L.; Silla, E.; Tuñón, I. J. Comp. Chem. 1994, 15, 1127-1138. (c) Cossi, M.; Scalmani, G.; Rega, N.; Barone, V. J. Chem. Phys. 2002, 117, 43-54. 


$\begin{array}{rrrrrrrr}\mathrm{Cl} & -2.99842 & 2.265128 & -0.98069 & \mathrm{H} & 2.73244 & -0.85917 & 0.126819 \\ \mathrm{Cl} & -1.51018 & 5.03832 & -0.49635 & \mathrm{~N} & 1.473454 & -1.9688 & -1.58627 \\ \mathrm{Cl} & 1.508632 & 5.039051 & 0.496195 & \mathrm{H} & 1.048567 & -1.26267 & -0.94896 \\ \mathrm{Cl} & 2.998237 & 2.266565 & 0.980526 & \mathrm{~N} & 3.430712 & -3.19007 & -2.03625 \\ \mathrm{~N} & -3.32447 & -1.62602 & 0.274099 & \mathrm{H} & 4.281723 & -3.56515 & -1.62524 \\ \mathrm{C} & -2.74235 & -2.26791 & 1.295638 & \mathrm{H} & 2.87989 & -3.91231 & -2.49295 \\ \mathrm{H} & -2.7319 & -0.85968 & -0.12717 & \mathrm{C} & 0.70167 & -2.46125 & -2.73567 \\ \mathrm{~N} & -1.47309 & -1.969 & 1.58629 & \mathrm{H} & 1.418277 & -2.8246 & -3.48286 \\ \mathrm{H} & -1.04829 & -1.26268 & 0.949044 & \mathrm{C} & -0.22192 & -3.61948 & -2.33001 \\ \mathrm{~N} & -3.43043 & -3.19022 & 2.036262 & \mathrm{H} & -0.75985 & -4.00368 & -3.20524 \\ \mathrm{H} & -4.28118 & -3.56558 & 1.624992 & \mathrm{H} & 0.346982 & -4.44736 & -1.89038 \\ \mathrm{H} & -2.87962 & -3.91227 & 2.49327 & \mathrm{H} & -0.9572 & -3.28245 & -1.59234 \\ \mathrm{C} & -0.70153 & -2.46111 & 2.735984 & \mathrm{C} & -0.07342 & -1.28901 & -3.35012 \\ \mathrm{H} & -1.41826 & -2.82415 & 3.483202 & \mathrm{H} & 0.615324 & -0.51524 & -3.70579 \\ \mathrm{C} & 0.222074 & -3.61953 & 2.330865 & \mathrm{H} & -0.66802 & -1.64067 & -4.20061 \\ \mathrm{H} & 0.759882 & -4.00343 & 3.2063 & \mathrm{H} & -0.74207 & -0.84113 & -2.60733 \\ \mathrm{H} & -0.34677 & -4.44756 & 1.891439 & \mathrm{C} & 4.729577 & -1.71988 & 0.129569 \\ \mathrm{H} & 0.957464 & -3.28277 & 1.593174 & \mathrm{H} & 5.071547 & -2.75157 & -0.03871 \\ \mathrm{C} & 0.073543 & -1.28871 & 3.350145 & \mathrm{C} & 4.823648 & -1.44308 & 1.633337 \\ \mathrm{H} & -0.6152 & -0.5148 & 3.705507 & \mathrm{H} & 4.231884 & -2.17112 & 2.197262 \\ \mathrm{H} & 0.668032 & -1.64012 & 4.200813 & \mathrm{H} & 5.866257 & -1.50977 & 1.962708 \\ \mathrm{H} & 0.742287 & -0.84105 & 2.607306 & \mathrm{H} & 4.44893 & -0.44105 & 1.866108 \\ \mathrm{C} & -4.72906 & -1.72047 & -0.12984 & \mathrm{C} & 5.610289 & -0.76174 & -0.68872 \\ \mathrm{H} & -5.07096 & -2.75218 & 0.038443 & \mathrm{H} & 6.661106 & -0.8584 & -0.39107 \\ \mathrm{C} & -4.82313 & -1.4437 & -1.63361 & \mathrm{H} & 5.536958 & -0.98002 & -1.75979 \\ \mathrm{H} & -4.23123 & -2.17166 & -2.1975 & \mathrm{H} & 5.293835 & 0.273673 & -0.52607\end{array}$

$\left(N, N^{\prime} \text {-diisopropylguanidinium }\right)_{2} \cdot \mathbf{Q}^{\bullet-}$ complex

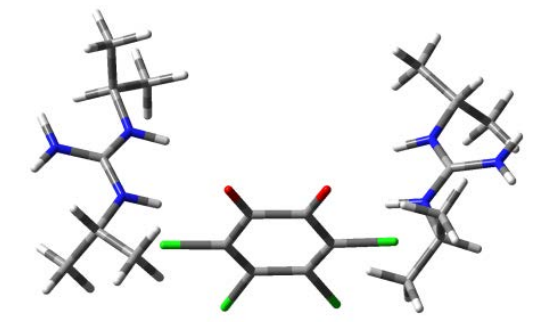

E(UM06-2X): -3102.807118

Counterpoise correction: 0.00435811 
PCM correction: -0.05899887

Cartesian coordinates:

\begin{tabular}{|c|c|c|c|c|c|c|c|}
\hline $\mathrm{C}$ & 3.735477 & -0.3897 & -0.26686 & $\mathrm{H}$ & -3.44778 & -4.76071 & 1.723379 \\
\hline $\mathrm{C}$ & 3.378768 & -1.74182 & 0.08334 & $\mathrm{H}$ & -1.8503 & -4.28224 & 1.123869 \\
\hline $\mathrm{C}$ & 2.057588 & -2.10474 & 0.2161 & $\mathrm{C}$ & -3.63867 & -4.58496 & -1.01432 \\
\hline $\mathrm{C}$ & 1.007852 & -1.15338 & 0.000033 & $\mathrm{H}$ & -4.30002 & -5.36634 & -0.63114 \\
\hline $\mathrm{C}$ & 1.372166 & 0.22059 & -0.38656 & $\mathrm{H}$ & -4.03937 & -4.23682 & -1.97185 \\
\hline $\mathrm{C}$ & 2.758617 & 0.555872 & -0.48855 & $\mathrm{H}$ & -2.65642 & -5.02619 & -1.207 \\
\hline $\mathrm{O}$ & 0.430673 & 1.040156 & -0.59171 & $\mathrm{~N}$ & -0.52548 & 3.303357 & 0.568216 \\
\hline $\mathrm{O}$ & -0.21745 & -1.4163 & 0.134039 & $\mathrm{C}$ & -1.30441 & 2.755769 & 1.49587 \\
\hline $\mathrm{Cl}$ & 1.583479 & -3.70363 & 0.650975 & $\mathrm{H}$ & 0.076394 & 2.653902 & 0.045122 \\
\hline $\mathrm{Cl}$ & 4.623449 & -2.89568 & 0.343227 & $\mathrm{~N}$ & -1.32018 & 1.429522 & 1.653244 \\
\hline $\mathrm{Cl}$ & 5.395724 & 0.031086 & -0.39461 & $\mathrm{H}$ & -0.80591 & 0.888492 & 0.950986 \\
\hline $\mathrm{Cl}$ & 3.137091 & 2.19374 & -0.88319 & $\mathrm{~N}$ & -2.10388 & 3.542558 & 2.246904 \\
\hline $\mathrm{N}$ & -2.67972 & -2.35964 & -0.51657 & $\mathrm{H}$ & -1.93068 & 4.533841 & 2.294517 \\
\hline $\mathrm{C}$ & -3.10248 & -1.35917 & -1.28517 & $\mathrm{H}$ & -2.68352 & 3.143738 & 2.966427 \\
\hline $\mathrm{H}$ & -1.69156 & -2.33462 & -0.24779 & $\mathrm{C}$ & -2.09969 & 0.686108 & 2.649723 \\
\hline $\mathrm{N}$ & -2.26982 & -0.37312 & -1.62284 & $\mathrm{H}$ & -2.22843 & 1.334943 & 3.524583 \\
\hline $\mathrm{H}$ & -1.35683 & -0.36322 & -1.15334 & $\mathrm{C}$ & -3.46497 & 0.297816 & 2.082556 \\
\hline $\mathrm{N}$ & -4.39248 & -1.33097 & -1.68522 & $\mathrm{H}$ & -4.05789 & -0.22931 & 2.835521 \\
\hline $\mathrm{H}$ & -4.95201 & -2.16728 & -1.63473 & $\mathrm{H}$ & -4.02818 & 1.174492 & 1.747038 \\
\hline $\mathrm{H}$ & -4.72217 & -0.59734 & -2.29039 & $\mathrm{H}$ & -3.32235 & -0.36932 & 1.224288 \\
\hline $\mathrm{C}$ & -2.61137 & 0.763799 & -2.48733 & $\mathrm{C}$ & -1.2962 & -0.5314 & 3.096418 \\
\hline $\mathrm{H}$ & -3.34567 & 0.410017 & -3.2216 & $\mathrm{H}$ & -0.33783 & -0.22869 & 3.526028 \\
\hline $\mathrm{C}$ & -3.20483 & 1.905787 & -1.66215 & $\mathrm{H}$ & -1.85561 & -1.08516 & 3.854844 \\
\hline $\mathrm{H}$ & -3.51597 & 2.72895 & -2.31176 & $\mathrm{H}$ & -1.09992 & -1.19335 & 2.246627 \\
\hline $\mathrm{H}$ & -4.07108 & 1.577053 & -1.07832 & $\mathrm{C}$ & -0.46225 & 4.727918 & 0.222061 \\
\hline $\mathrm{H}$ & -2.4448 & 2.281422 & -0.96759 & $\mathrm{H}$ & -1.4728 & 5.143775 & 0.329676 \\
\hline $\mathrm{C}$ & -1.37043 & 1.198873 & -3.25965 & $\mathrm{C}$ & -0.06116 & 4.826186 & -1.24637 \\
\hline $\mathrm{H}$ & -0.98067 & 0.376728 & -3.8649 & $\mathrm{H}$ & -0.79216 & 4.323716 & -1.88608 \\
\hline $\mathrm{H}$ & -1.62685 & 2.027141 & -3.92515 & $\mathrm{H}$ & 0.006048 & 5.873532 & -1.54848 \\
\hline $\mathrm{H}$ & -0.58742 & 1.527091 & -2.56992 & $\mathrm{H}$ & 0.917038 & 4.359558 & -1.40486 \\
\hline $\mathrm{C}$ & -3.50815 & -3.4529 & 0.005448 & $\mathrm{C}$ & 0.516731 & 5.47164 & 1.130683 \\
\hline $\mathrm{H}$ & -4.49778 & -3.03757 & 0.239634 & $\mathrm{H}$ & 0.535003 & 6.535472 & 0.880255 \\
\hline$C$ & -2.87207 & -3.93183 & 1.305967 & $\mathrm{H}$ & 0.254391 & 5.373818 & 2.189183 \\
\hline $\mathrm{H}$ & -2.83481 & -3.12497 & 2.043085 & $\mathrm{H}$ & 1.524228 & 5.065963 & 0.998628 \\
\hline
\end{tabular}


(2) ${ }_{2} \cdot \mathbf{Q}^{\bullet-}$ complex

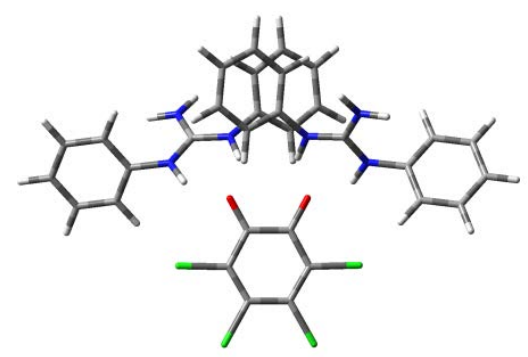

E(UB3LYP): -3555.96022230

Counterpoise correction: 0.00497453

Dispersion correction: -0.1830365

PCM correction: -0.05307481

Cartesian coordinates:

$\begin{array}{lrrrrrrr}\mathrm{C} & -5.70864 & 0.605306 & 0.560246 & \mathrm{O} & -1.28069 & 0.527159 & -0.4387 \\ \mathrm{C} & -5.08819 & -0.56617 & 0.111977 & \mathrm{O} & 1.280795 & 0.527212 & 0.438757 \\ \mathrm{C} & -5.83192 & -1.56195 & -0.53381 & \mathrm{Cl} & 2.96272 & 2.868601 & 1.098422 \\ \mathrm{C} & -7.20678 & -1.38905 & -0.71215 & \mathrm{Cl} & 1.46501 & 5.610967 & 0.554336 \\ \mathrm{C} & -7.83106 & -0.22034 & -0.26716 & \mathrm{Cl} & -1.46509 & 5.610906 & -0.55433 \\ \mathrm{C} & -7.08062 & 0.776665 & 0.362598 & \mathrm{Cl} & -2.9627 & 2.868481 & -1.09839 \\ \mathrm{H} & -5.11824 & 1.373097 & 1.049936 & \mathrm{C} & 5.70867 & 0.605309 & -0.56029 \\ \mathrm{H} & -5.33334 & -2.45184 & -0.90641 & \mathrm{C} & 5.088225 & -0.56618 & -0.11204 \\ \mathrm{H} & -7.78431 & -2.15972 & -1.21301 & \mathrm{C} & 5.831959 & -1.56199 & 0.533684 \\ \mathrm{H} & -8.89765 & -0.08466 & -0.41587 & \mathrm{C} & 7.206832 & -1.3891 & 0.711993 \\ \mathrm{H} & -7.5617 & 1.687678 & 0.704277 & \mathrm{C} & 7.831106 & -0.22038 & 0.267026 \\ \mathrm{~N} & -3.67062 & -0.72235 & 0.283414 & \mathrm{C} & 7.080656 & 0.77665 & -0.36268 \\ \mathrm{C} & -3.10139 & -1.59453 & 1.130042 & \mathrm{H} & 5.118261 & 1.373122 & -1.04993 \\ \mathrm{H} & -3.02874 & -0.06523 & -0.1813 & \mathrm{H} & 5.333385 & -2.45189 & 0.906269 \\ \mathrm{~N} & -1.76 & -1.63231 & 1.211407 & \mathrm{H} & 7.784368 & -2.15979 & 1.212806 \\ \mathrm{H} & -1.27048 & -0.89707 & 0.677447 & \mathrm{H} & 8.897703 & -0.08471 & 0.415703 \\ \mathrm{C} & -1.00065 & -2.46841 & 2.089498 & \mathrm{H} & 7.561733 & 1.687672 & -0.70434 \\ \mathrm{C} & -0.10107 & -1.86939 & 2.979583 & \mathrm{~N} & 3.67064 & -0.72234 & -0.28344 \\ \mathrm{C} & -1.11365 & -3.86445 & 2.032909 & \mathrm{C} & 3.101375 & -1.59451 & -1.13006 \\ \mathrm{C} & 0.671093 & -2.6689 & 3.824302 & \mathrm{H} & 3.028783 & -0.06521 & 0.181289 \\ \mathrm{H} & -0.00649 & -0.78848 & 3.001395 & \mathrm{~N} & 1.759983 & -1.63229 & -1.21136 \\ \mathrm{C} & -0.35162 & -4.65496 & 2.897752 & \mathrm{H} & 1.27048 & -0.89704 & -0.67738 \\ \mathrm{H} & -1.7722 & -4.32784 & 1.304301 & \mathrm{C} & 1.000618 & -2.46838 & -2.08944 \\ \mathrm{C} & 0.540021 & -4.0605 & 3.794489 & \mathrm{C} & 0.101001 & -1.86937 & -2.97949\end{array}$




$\begin{array}{lrrr}\mathrm{H} & 1.366731 & -2.20095 & 4.513903 \\ \mathrm{H} & -0.44629 & -5.73579 & 2.857577 \\ \mathrm{H} & 1.132285 & -4.67791 & 4.46248 \\ \mathrm{~N} & -3.8636 & -2.4191 & 1.870506 \\ \mathrm{H} & -4.86587 & -2.29745 & 1.872912 \\ \mathrm{H} & -3.43872 & -2.98256 & 2.591952 \\ \mathrm{C} & -0.67464 & 4.100868 & -0.25303 \\ \mathrm{C} & 0.67462 & 4.100896 & 0.253041 \\ \mathrm{C} & 1.330041 & 2.905754 & 0.493184 \\ \mathrm{C} & 0.699075 & 1.639476 & 0.247753 \\ \mathrm{C} & -0.69901 & 1.639447 & -0.24771 \\ \mathrm{C} & -1.33002 & 2.905699 & -0.49315\end{array}$

(2) ${ }_{2} \cdot \mathbf{Q}^{\bullet-}$ complex

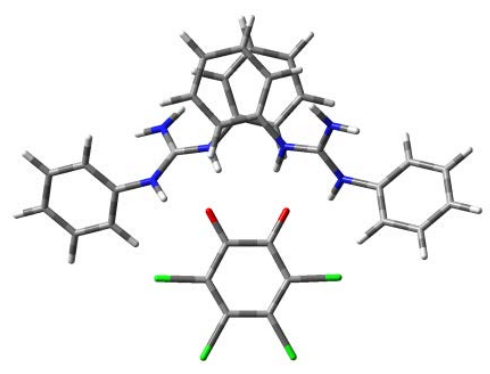

E(UM06-2X): -3555.11972241

Counterpoise correction: 0.00570859

PCM correction: -0.05317968

Cartesian coordinates:

$\begin{array}{lrrr}\mathrm{C} & 1.113665 & -3.86442 & -2.03289 \\ \mathrm{C} & -0.67117 & -2.66889 & -3.8242 \\ \mathrm{H} & 0.006383 & -0.78846 & -3.00128 \\ \mathrm{C} & 0.351635 & -4.65493 & -2.89772 \\ \mathrm{H} & 1.772261 & -4.3278 & -1.30432 \\ \mathrm{C} & -0.54006 & -4.06048 & -3.79442 \\ \mathrm{H} & -1.36684 & -2.20094 & -4.51377 \\ \mathrm{H} & 0.446343 & -5.73576 & -2.85758 \\ \mathrm{H} & -1.13232 & -4.6779 & -4.4624 \\ \mathrm{~N} & 3.863532 & -2.41911 & -1.87053 \\ \mathrm{H} & 4.865807 & -2.29748 & -1.87299 \\ \mathrm{H} & 3.438607 & -2.98258 & -2.59195\end{array}$

$\begin{array}{lrrrlrrr}\mathrm{C} & -5.46514 & 0.947517 & 0.682763 & \mathrm{O} & -1.17141 & 0.547016 & -0.67057 \\ \mathrm{C} & -5.02892 & -0.2414 & 0.099711 & \mathrm{O} & 1.171427 & 0.54706 & 0.670561 \\ \mathrm{C} & -5.93564 & -1.1254 & -0.48759 & \mathrm{Cl} & 2.853913 & 2.857082 & 1.312744 \\ \mathrm{C} & -7.29565 & -0.82324 & -0.46951 & \mathrm{Cl} & 1.431514 & 5.59887 & 0.618326 \\ \mathrm{C} & -7.7395 & 0.364351 & 0.110512 & \mathrm{Cl} & -1.43171 & 5.598813 & -0.61832 \\ \mathrm{C} & -6.82498 & 1.250612 & 0.678237 & \mathrm{Cl} & -2.854 & 2.856971 & -1.31274 \\ \mathrm{H} & -4.74135 & 1.625124 & 1.125133 & \mathrm{C} & 5.465142 & 0.947611 & -0.68279 \\ \mathrm{H} & -5.57143 & -2.03067 & -0.96559 & \mathrm{C} & 5.028933 & -0.2413 & -0.09972 \\ \mathrm{H} & -8.0048 & -1.50794 & -0.92266 & \mathrm{C} & 5.935671 & -1.12528 & 0.487591 \\ \mathrm{H} & -8.79795 & 0.602405 & 0.11415 & \mathrm{C} & 7.295673 & -0.82309 & 0.469519 \\ \mathrm{H} & -7.1696 & 2.178241 & 1.122905 & \mathrm{C} & 7.739511 & 0.36449 & -0.11052\end{array}$




$\begin{array}{lrrr}\mathrm{N} & -3.62888 & -0.54172 & 0.083586 \\ \mathrm{C} & -3.07311 & -1.53722 & 0.783261 \\ \mathrm{H} & -2.98267 & 0.086229 & -0.40826 \\ \mathrm{~N} & -1.74403 & -1.67757 & 0.755661 \\ \mathrm{H} & -1.23046 & -0.9089 & 0.296647 \\ \mathrm{C} & -0.99719 & -2.74101 & 1.335128 \\ \mathrm{C} & 0.142627 & -2.41738 & 2.074784 \\ \mathrm{C} & -1.34264 & -4.07456 & 1.10653 \\ \mathrm{C} & 0.929454 & -3.43966 & 2.598517 \\ \mathrm{H} & 0.40561 & -1.37241 & 2.220922 \\ \mathrm{C} & -0.56269 & -5.08746 & 1.659698 \\ \mathrm{H} & -2.19068 & -4.31708 & 0.47217 \\ \mathrm{C} & 0.572935 & -4.77458 & 2.404785 \\ \mathrm{H} & 1.81327 & -3.18935 & 3.176668 \\ \mathrm{H} & -0.83242 & -6.12427 & 1.485089 \\ \mathrm{H} & 1.179312 & -5.56735 & 2.830571 \\ \mathrm{~N} & -3.8416 & -2.37921 & 1.489186 \\ \mathrm{H} & -4.82697 & -2.17883 & 1.587756 \\ \mathrm{H} & -3.40625 & -3.01616 & 2.140811 \\ \mathrm{C} & -0.65586 & 4.100599 & -0.29856 \\ \mathrm{C} & 0.65575 & 4.100625 & 0.298535 \\ \mathrm{C} & 1.277886 & 2.912943 & 0.606502 \\ \mathrm{C} & 0.65007 & 1.649499 & 0.346235 \\ \mathrm{C} & -0.65009 & 1.649474 & -0.34626 \\ \mathrm{C} & -1.27796 & 2.912894 & -0.60653\end{array}$

$\begin{array}{lrrr}\mathrm{C} & 6.824975 & 1.250728 & -0.67827 \\ \mathrm{H} & 4.741339 & 1.625199 & -1.12517 \\ \mathrm{H} & 5.571474 & -2.03055 & 0.965609 \\ \mathrm{H} & 8.004836 & -1.50778 & 0.922679 \\ \mathrm{H} & 8.797956 & 0.602562 & -0.11416 \\ \mathrm{H} & 7.169584 & 2.178356 & -1.12295 \\ \mathrm{~N} & 3.628901 & -0.54165 & -0.0836 \\ \mathrm{C} & 3.073147 & -1.53717 & -0.78325 \\ \mathrm{H} & 2.982677 & 0.086304 & 0.40824 \\ \mathrm{~N} & 1.744072 & -1.67754 & -0.75566 \\ \mathrm{H} & 1.230482 & -0.90887 & -0.29666 \\ \mathrm{C} & 0.997254 & -2.74099 & -1.33512 \\ \mathrm{C} & -0.14255 & -2.41741 & -2.07481 \\ \mathrm{C} & 1.342734 & -4.07454 & -1.10649 \\ \mathrm{C} & -0.92934 & -3.43972 & -2.59854 \\ \mathrm{H} & -0.40555 & -1.37244 & -2.22098 \\ \mathrm{C} & 0.562822 & -5.08747 & -1.65965 \\ \mathrm{H} & 2.190765 & -4.31702 & -0.4721 \\ \mathrm{C} & -0.5728 & -4.77462 & -2.40477 \\ \mathrm{H} & -1.81315 & -3.18944 & -3.17672 \\ \mathrm{H} & 0.832567 & -6.12426 & -1.48502 \\ \mathrm{H} & -1.17915 & -5.56742 & -2.83055 \\ \mathrm{~N} & 3.841644 & -2.37917 & -1.48916 \\ \mathrm{H} & 4.827008 & -2.17879 & -1.58773 \\ \mathrm{H} & 3.406299 & -3.01614 & -2.14077\end{array}$

Bis-amidinium $\cdot \mathbf{Q}^{\bullet-}$ complex

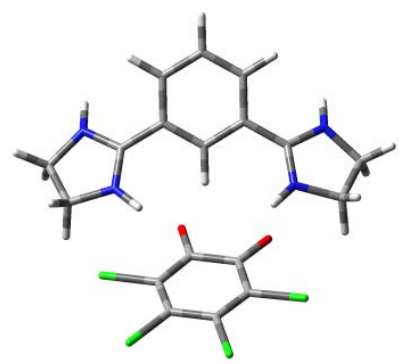

E(UB3LYP): -2905.629578

Counterpoise correction: 0.0020886 
Dispersion correction: -0.11208832

PCM correction: -0.06835953

Cartesian coordinates:

$\begin{array}{lrrrrrrr}\mathrm{C} & -2.987137 & 0.013695 & -0.08827 & \mathrm{C} & -3.075525 & 4.658001 & -1.043567 \\ \mathrm{C} & -3.542817 & 1.222789 & 0.347345 & \mathrm{H} & -4.819149 & 3.523272 & -0.363102 \\ \mathrm{C} & -4.583267 & 1.223465 & 1.291484 & \mathrm{H} & -1.009668 & 4.630847 & -0.243592 \\ \mathrm{C} & -5.066046 & 0.017796 & 1.797836 & \mathrm{H} & -1.107088 & 4.175818 & -1.953482 \\ \mathrm{C} & -4.526597 & -1.190068 & 1.357488 & \mathrm{H} & -3.448367 & 4.752283 & -2.068674 \\ \mathrm{C} & -3.486582 & -1.196212 & 0.412497 & \mathrm{H} & -3.194799 & 5.614644 & -0.532035 \\ \mathrm{H} & -2.169941 & 0.01619 & -0.800106 & \mathrm{~N} & -1.694937 & -2.664136 & -0.466472 \\ \mathrm{H} & -4.990238 & 2.16052 & 1.658649 & \mathrm{~N} & -3.647969 & -3.61707 & -0.089325 \\ \mathrm{H} & -5.852223 & 0.019492 & 2.545007 & \mathrm{~N} & -1.802236 & 2.714975 & -0.583335 \\ \mathrm{H} & -4.891219 & -2.121859 & 1.778572 & \mathrm{~N} & -3.811347 & 3.58803 & -0.336619 \\ \mathrm{C} & -2.927727 & -2.478587 & -0.055889 & \mathrm{C} & 3.886277 & 0.504774 & 0.21216 \\ \mathrm{C} & -1.458824 & -4.083873 & -0.789862 & \mathrm{C} & 3.544887 & -0.888344 & 0.09131 \\ \mathrm{H} & -0.918692 & -1.932456 & -0.414075 & \mathrm{C} & 2.227284 & -1.27127 & -0.108804 \\ \mathrm{C} & -2.885668 & -4.686963 & -0.765831 & \mathrm{C} & 1.165457 & -0.317703 & -0.204532 \\ \mathrm{H} & -4.657996 & -3.604216 & -0.083408 & \mathrm{C} & 1.516729 & 1.118388 & -0.075073 \\ \mathrm{H} & -0.964338 & -4.1797 & -1.75769 & \mathrm{C} & 2.900676 & 1.469755 & 0.131325 \\ \mathrm{H} & -0.812273 & -4.520818 & -0.023595 & \mathrm{O} & 0.615882 & 1.99938 & -0.144884 \\ \mathrm{H} & -2.941281 & -5.620615 & -0.20373 & \mathrm{O} & -0.055436 & -0.625447 & -0.393526 \\ \mathrm{H} & -3.284893 & -4.851219 & -1.772261 & \mathrm{Cl} & 1.803131 & -2.960616 & -0.263336 \\ \mathrm{C} & -3.035555 & 2.496023 & -0.195528 & \mathrm{Cl} & 4.797171 & -2.07808 & 0.197627 \\ \mathrm{C} & -1.622534 & 4.122221 & -0.993277 & \mathrm{Cl} & 5.540491 & 0.952959 & 0.460046 \\ \mathrm{H} & -0.931419 & 2.137199 & -0.379786 & \mathrm{Cl} & 3.26787 & 3.159457 & 0.271895\end{array}$

Bis-amidinium $\cdot \mathbf{Q}^{\bullet-}$ complex

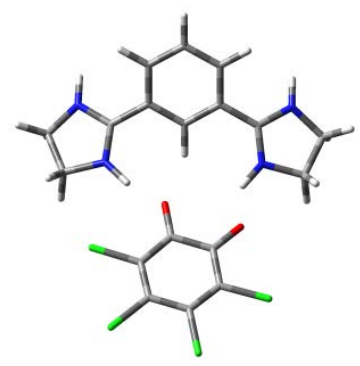

E(UM06-2X): -2905.065064

Counterpoise correction: 0.00212957 
PCM correction: -0.06712119

Cartesian coordinates:

$\begin{array}{rrrrrrrr}\mathrm{C} & -2.983885 & 0.039459 & -0.126969 & \mathrm{C} & -2.939333 & 4.688075 & -0.892983 \\ \mathrm{C} & -3.524011 & 1.230686 & 0.355295 & \mathrm{H} & -4.719339 & 3.607999 & -0.222617 \\ \mathrm{C} & -4.556021 & 1.216057 & 1.299524 & \mathrm{H} & -0.89803 & 4.54086 & -0.055102 \\ \mathrm{C} & -5.050937 & 0.001951 & 1.765475 & \mathrm{H} & -0.974451 & 4.192653 & -1.792803 \\ \mathrm{C} & -4.519566 & -1.193394 & 1.289873 & \mathrm{H} & -3.302998 & 4.808665 & -1.917529 \\ \mathrm{C} & -3.486047 & -1.172634 & 0.347393 & \mathrm{H} & -3.027114 & 5.636634 & -0.36343 \\ \mathrm{H} & -2.177188 & 0.058576 & -0.853024 & \mathrm{~N} & -1.665227 & -2.594275 & -0.500506 \\ \mathrm{H} & -4.947825 & 2.14709 & 1.698811 & \mathrm{~N} & -3.621216 & -3.571244 & -0.245587 \\ \mathrm{H} & -5.837139 & -0.013471 & 2.511965 & \mathrm{~N} & -1.750122 & 2.688512 & -0.525051 \\ \mathrm{H} & -4.885281 & -2.138348 & 1.681235 & \mathrm{~N} & -3.709588 & 3.63165 & -0.212469 \\ \mathrm{C} & -2.910285 & -2.437612 & -0.143079 & \mathrm{C} & 3.866079 & 0.441508 & 0.180661 \\ \mathrm{C} & -1.407039 & -4.002198 & -0.838289 & \mathrm{C} & 3.455918 & -0.936223 & 0.147647 \\ \mathrm{H} & -0.905126 & -1.835877 & -0.395281 & \mathrm{C} & 2.127087 & -1.263342 & -0.018318 \\ \mathrm{C} & -2.829166 & -4.589095 & -0.957025 & \mathrm{C} & 1.113855 & -0.267019 & -0.159271 \\ \mathrm{H} & -4.630808 & -3.556636 & -0.278967 & \mathrm{C} & 1.532288 & 1.147316 & -0.114516 \\ \mathrm{H} & -0.826548 & -4.078755 & -1.757638 & \mathrm{C} & 2.934173 & 1.442848 & 0.052781 \\ \mathrm{H} & -0.843988 & -4.462607 & -0.021416 & \mathrm{O} & 0.674676 & 2.055156 & -0.224403 \\ \mathrm{H} & -2.92438 & -5.564447 & -0.480646 & \mathrm{O} & -0.116701 & -0.52409 & -0.320677 \\ \mathrm{H} & -3.161642 & -4.656759 & -1.997461 & \mathrm{Cl} & 1.613903 & -2.917895 & -0.069514 \\ \mathrm{C} & -2.981223 & 2.508631 & -0.135031 & \mathrm{Cl} & 4.639407 & -2.167855 & 0.317058 \\ \mathrm{C} & -1.510841 & 4.103976 & -0.848232 & \mathrm{Cl} & 5.531846 & 0.813651 & 0.383552 \\ \mathrm{H} & -0.890541 & 2.078099 & -0.365902 & \mathrm{Cl} & 3.37516 & 3.104531 & 0.082205\end{array}$

\section{B. Kinetic analysis of electron transfer to o-chloranil promoted by}

\section{HBDs}

\section{2b.1 Introduction}

Catalysis of organic reactions by HBDs is achieved through stabilization of transition structures, which decreases the energy barriers to these reactions. ${ }^{33}$ In Part A, we established that

\footnotetext{
${ }^{33}$ (a) Taylor, M. S.; Jacobsen, E. N. Angew. Chem. Int. Ed. 2006, 45, 1520-1543. (b) Knowles, R. R.; Jacobsen, E. N. Proc. Natl. Acad. Sci. USA 2010, 107, 20678-20685
} 
dicatonic HBDs can favorably influence the thermodynamics of electron transfer to an electrondeficient quinone through tight binding to $\mathbf{Q}^{\bullet-}$. Herein, we report the effects of HBDs on the kinetics of electron transfer, and the correlation of such effects with the thermodynamic trends measured in the electrochemical experiments.

\section{2b.2 Design of kinetic experiments}

The question of kinetic effects was addressed using UV-vis spectrophotometry to monitor simple, homogeneous electron transfer reactions between $\mathbf{Q}$ and several electron donors, promoted by HBDs (Figure 2.62). Specifically, we examined an electron transfer between $\mathbf{Q}$ and ferrocene derivatives, which stoichiometrically produce salts of $\mathrm{HBD} \cdot \mathbf{Q}^{\bullet-}$ and the ferrocenium product. We opted to use ferrocenes as electron donors due to the distinct UV-vis absorption that is presented by their corresponding ferrocenium products, providing a convenient handle for monitoring the progress of the electron transfer reaction.

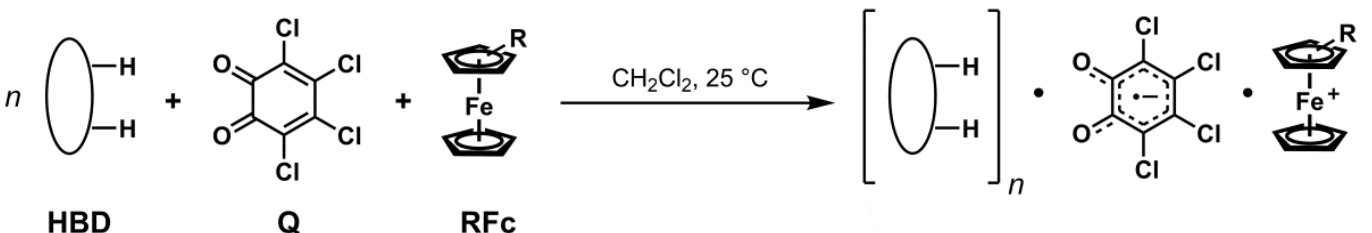

Figure 2.62. Homogeneous electron transfer between $\mathbf{Q}$ and ferrocene derviatives (RFc) promoted by HBDs.

Because the HBDs were found to span a broad range of reactivity, multiple electron donors with varying reduction potentials were required for this study (Figure 2.63). 


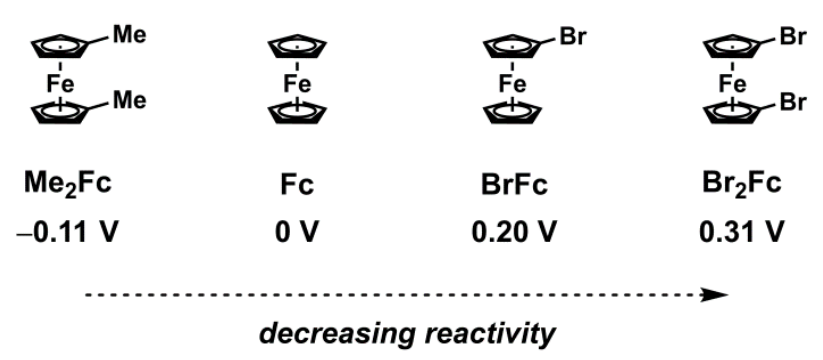

Figure 2.63. Series of ferrocene derivatives used as electron donors in this study. ${ }^{34}$

\section{2b.3 Determination of kinetic order in HBD for electron transfer reactions}

Our mechanistic analyses of the electrochemical data rested on our ability to determine the correct binding stoichiometries between the HBDs and $\mathbf{Q}^{\bullet-}$ (section 2a.5). Therefore, we sought independent verification for these analyses through determination of the kinetic order in HBD. These data would provide information required to assign the transition-state stoichiometry, in addition to offering evidence in support of a specific rate-limiting step within a pathway for HBD-coupled electron transfer.

The electron transfer between $\mathbf{Q}$ and bromoferrocene $(\mathbf{B r F c})$, promoted by dialkylguanidinium salt 1, was examined under conditions equimolar in $\mathbf{Q}$ and $\mathbf{B r F c}$, varying the excess of 1 . The instantaneous rates at 5\% yield, applying an initial rates assumption, were then used to determine the kinetic order in 1 (Figure 2.64). Our analysis reveals that this electron transfer is second-order in HBD. This result is consistent with the electrochemical experiments indicating that two cationic HBDs act in tandem to promote electron transfer. The order in $\mathbf{1}$ also indicates that the second binding event is rate-determining in HBD-coupled electron transfer.

\footnotetext{
${ }^{34}$ Ferrocene reduction potentials: Daeneke, T.; Mozer, A. J.; Uemura, Y.; Makuta, S.; Fekete, M.; Tachibana, Y.; Koumura, N.; Bach, U.; Spiccia, L. Dye Regeneration Kinetics in Dye-Sensitized Solar Cells. J. Am. Chem. Soc. 2012, 134, 16925-16928.
} 

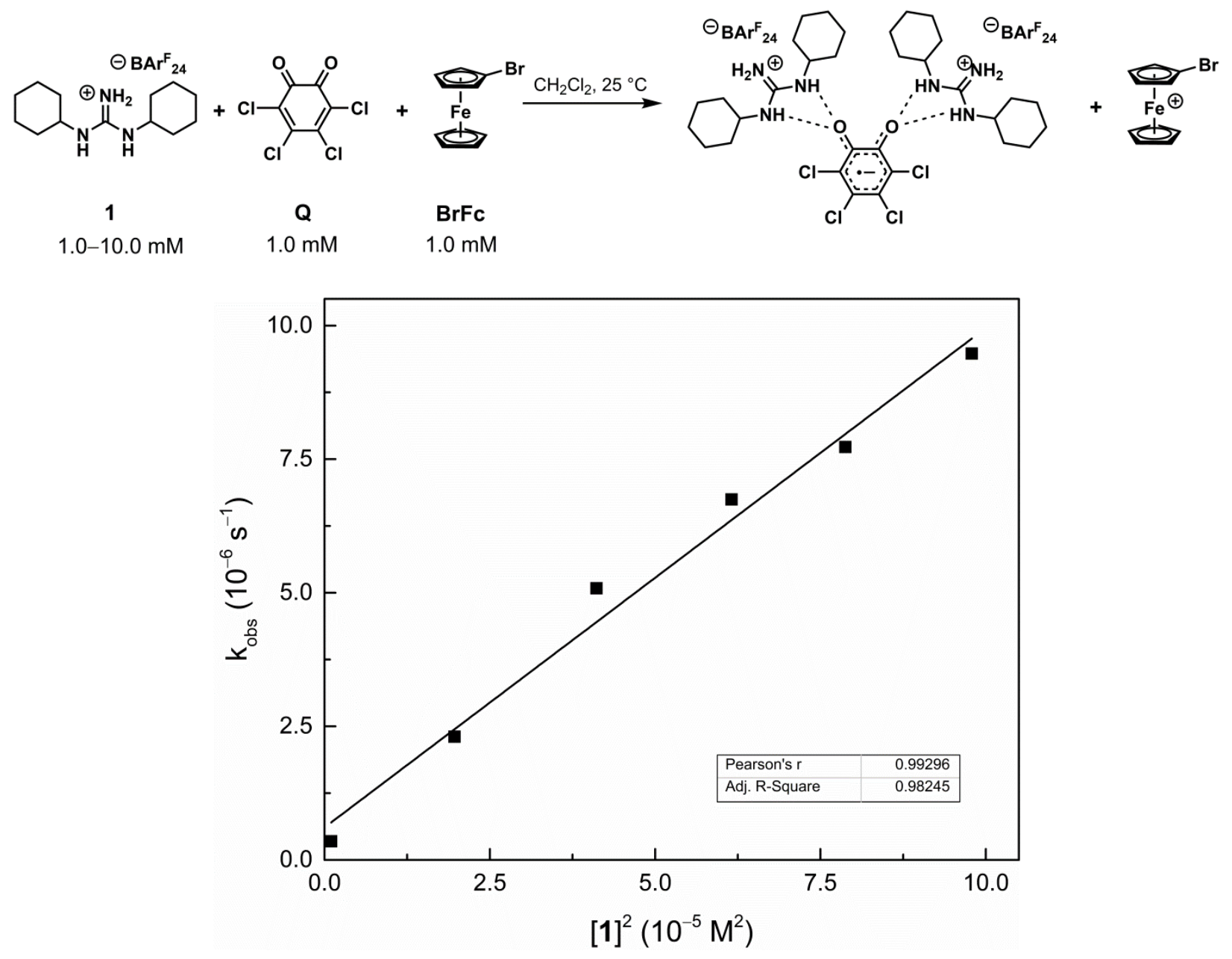

Figure 2.64. Second order plot of initial rate constants $\left(\mathrm{k}_{\mathrm{obs}}\right)$ vs. $[1]^{2}$ for electron transfer from BrFc $(1.0 \mathrm{mM})$ to $\mathbf{Q}(1.0 \mathrm{mM})$, promoted by $\mathbf{1}(1.0-10.0 \mathrm{mM})$ in $\mathrm{CH}_{2} \mathrm{Cl}_{2}$ at $25{ }^{\circ} \mathrm{C}$ under $\mathrm{N}_{2}\left(\mathrm{R}^{2}\right.$ $=0.98245)$.

In HBD-coupled electron transfer mediated by diphenylguanidinium salt $\mathbf{2}$, a significant improvement in reaction rate is observed. To compensate for this enhanced acceleration, 1,1'dibromoferrocene $\left(\mathbf{B r}_{2} \mathbf{F c}\right)$ - a weaker electron donor-was used. Consistent with the kinetic results obtained with $\mathbf{1}$ and analogous to the thermodynamic electrochemical results, the rate of electron transfer exhibits a second-order dependence on 2 (Figure 2.65), indicative of 2:1 stoichiometry between $\mathbf{2}$ and $\mathbf{Q}$ in the transition state. 

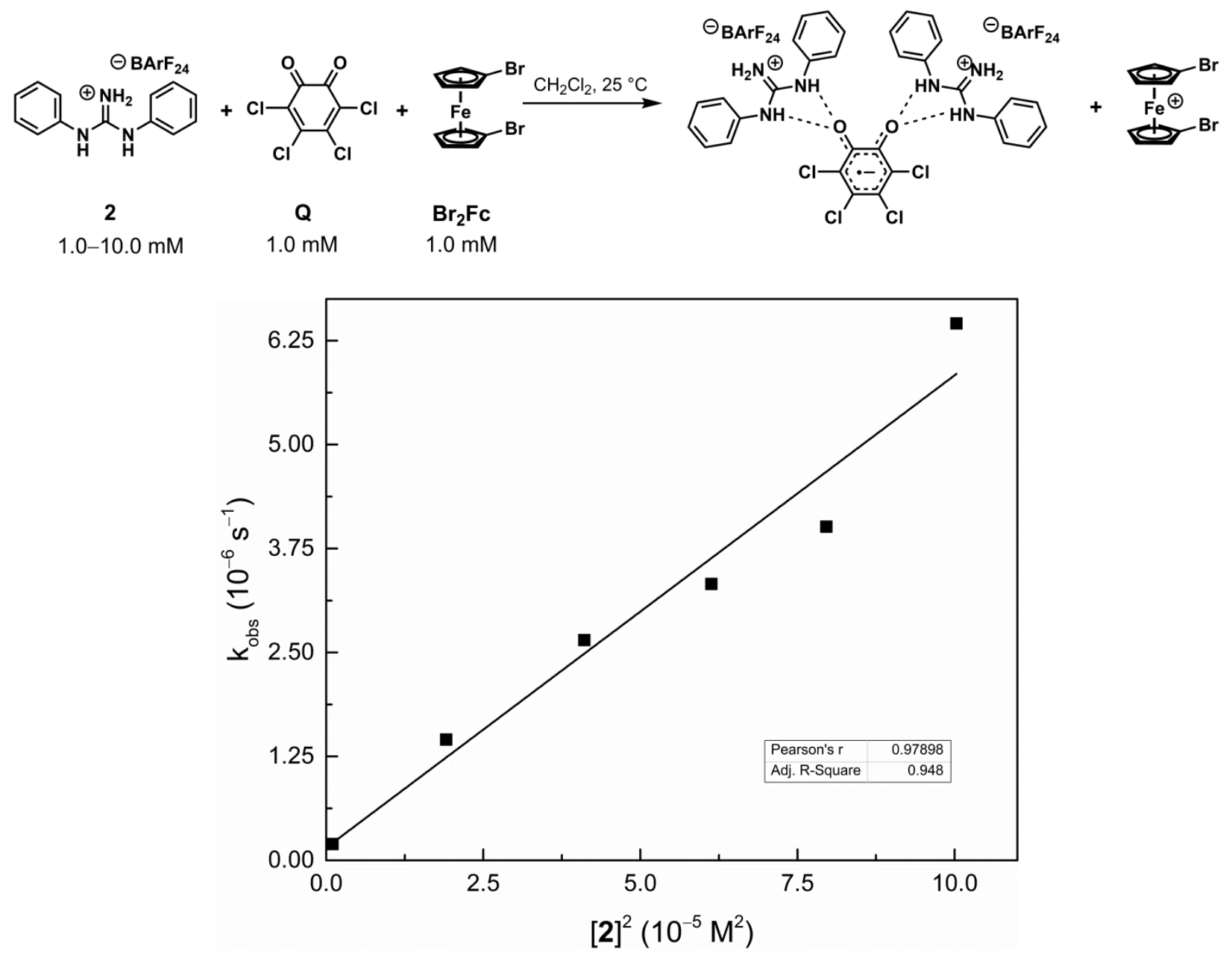

Figure 2.65. Second-order plot of initial rate constants $\left(\mathrm{k}_{\mathrm{obs}}\right)$ vs. $[2]^{2}$ for electron transfer from $\mathbf{B r}_{2} \mathbf{F c}(1.0 \mathrm{mM})$ to $\mathbf{Q}(1.0 \mathrm{mM})$, promoted by $2(1.0-10 \mathrm{mM})$ in $\mathrm{CH}_{2} \mathrm{Cl}_{2}$ at $25{ }^{\circ} \mathrm{C}$ under $\mathrm{N}_{2}\left(\mathrm{R}^{2}=\right.$ 0.948)

Electron transfer mediated by urea $\mathbf{3}$ exhibits significantly depressed reaction rates, requiring the use of 1,1'-dimethylferrocene $\left(\mathbf{M e}_{2} \mathbf{F c}\right)$, a stronger electron donor. In contrast with HBDs 1 and 2, the reaction mediated by urea $\mathbf{3}$ is found to be first-order in HBD (Figure 2.66). This result indicates that a 1:1 complex involving 3 is relevant to the rate-determining step (rds), but does not exclude the formation of a 2:1 complex over the entire reaction course. One likely explanation for this divergence in kinetic order is a change in rate-determining step upon use of a neutral HBD (Figure 2.67). When guanidinium salts $\mathbf{1}$ and $\mathbf{2}$ are used, the HBD·Q complex 
formed by pre-association is cationic and more strongly oxidizing-electron transfer to this complex is likely fast (Figure 2.67A). In the case of neutral 3, which forms a neutral HBD.Q complex, electron transfer occurs at a slower rate (Figure 2.67B). If this electron transfer, as opposed to the association of $\mathbf{3}$ with the $\mathbf{3} \cdot \mathbf{Q}^{--}$complex, is rate-determining, the reaction would exhibit a first-order dependence on $\mathbf{3}$. This would not exclude the formation of the $(\mathbf{3})_{2} \cdot \mathbf{Q}^{-}$ complex in a post-rate-limiting step.
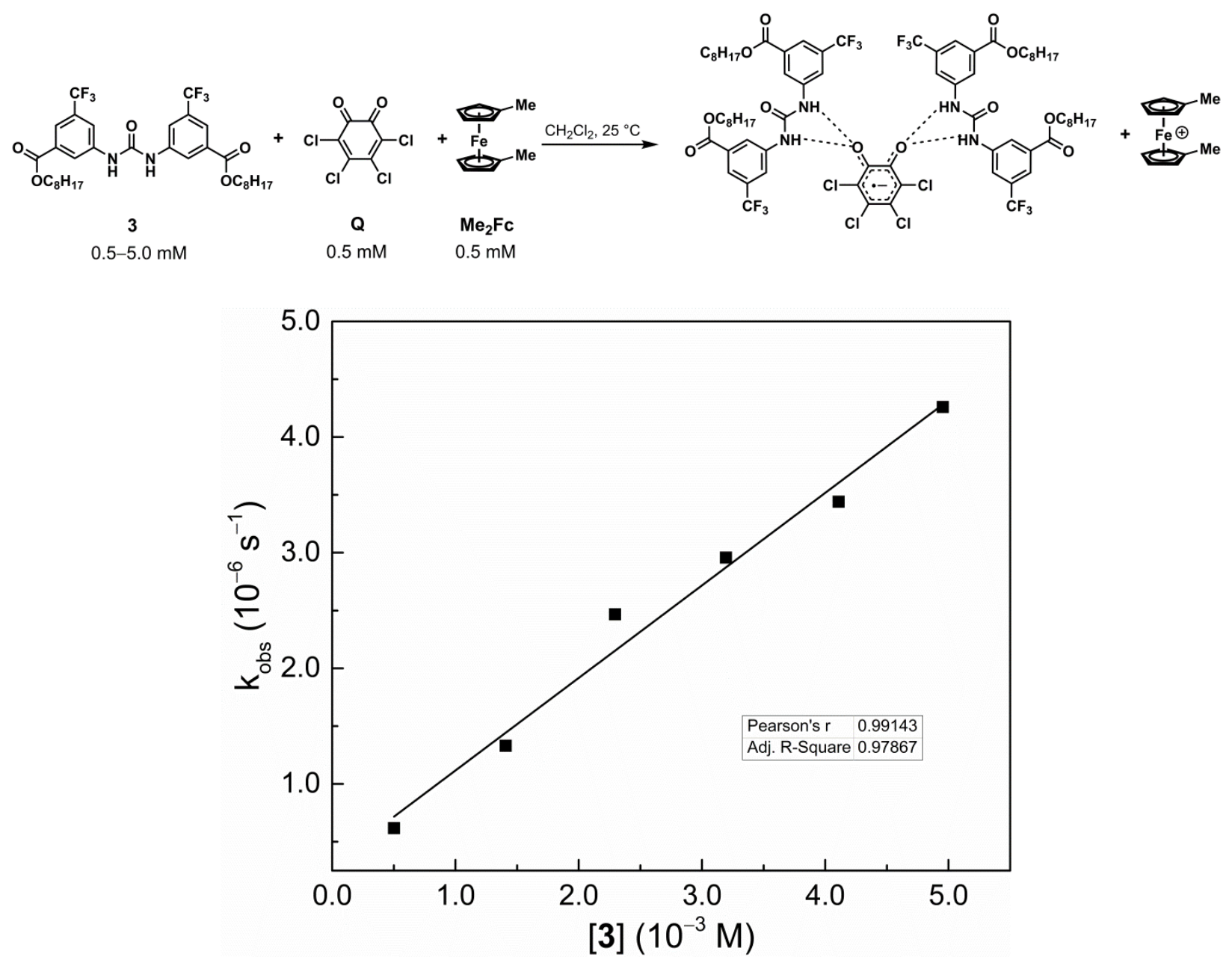

Figure 2.66. First-order plot of initial rate constants $\left(\mathrm{k}_{\mathrm{obs}}\right)$ vs. [3] for electron transfer from $\mathbf{M e}_{2} \mathbf{F c}(0.5 \mathrm{mM})$ to $\mathbf{Q}(0.5 \mathrm{mM})$, promoted by $3(0.5-5.0 \mathrm{mM})$ in $\mathrm{CH}_{2} \mathrm{Cl}_{2}$ at $25{ }^{\circ} \mathrm{C}$ under $\mathrm{N}_{2}\left(\mathrm{R}^{2}\right.$ $=0.97867)$ 
(a)
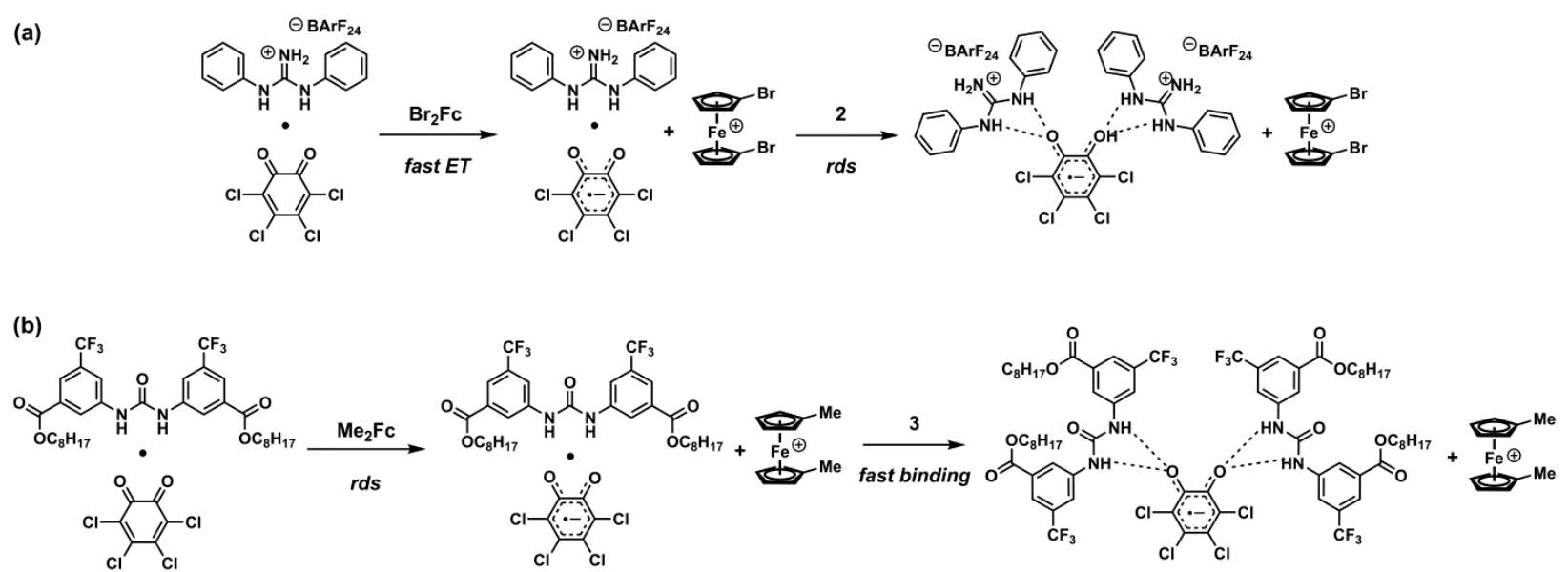

Figure 2.67. Proposed mechanisms for HBD-coupled electron transfer involving (a) a ratedetermining binding step using 2 (and monocationic HBDs). (b) rate-determining electron transfer using neutral 3.

\section{2b.4 Method of continuous variations for determination of $4 \cdot Q$ stoichiometry}

Determination of order of reaction in $\mathbf{4}$ proved challenging for practical reasons, largely due to the inherent reactivity observed with this HBD. Because $\mathbf{4}$ enhances the rate of electron transfer to such a large extent, measuring the rates at $5 \%$ conversion was not possible. Under pseudo-first-order conditions using a large excess of $\mathbf{4}$, the rates of electron transfer to $\mathbf{B r}_{2} \mathbf{F c}$ were also too rapid to obtain data at low conversion required to determine the kinetic order. Attempts to use a weaker electron donor also proved unsuccessful, as the products of this electron transfer appeared to be unstable under the conditions required for these experiments.

We therefore opted to use the method of continuous variation to determine the stoichiometry between $\mathbf{4}$ and $\mathbf{Q}^{\bullet-}$ over the course of a homogeneous electron transfer. Binding between $\mathbf{4}$ and $\mathbf{Q}$ induces a shift in the absorbance of $\mathbf{Q}$, and under the conditions required for this analysis, this shift interferes with the absorbance associated with $\mathbf{B r}_{2} \mathbf{F c}^{+}$. Instead, we used $N$-methylphenothiazine (MePTZ), an organic electron donor with a reduction potential 
comparable to that of $\mathbf{B r}_{2} \mathbf{F c}{ }^{35}$ A series of electron transfer reactions between $\mathbf{Q}$ and $\mathbf{M e P T Z}$ (used in excess) were carried out, varying the mole fractions of $\mathbf{4}$ and $\mathbf{Q}$. The amount of $\mathbf{M e P T Z}^{\bullet+}$ generated is directly proportional to the amount of $\mathbf{Q}^{\cdot-}$ that is complexed to 4 as a result of the electron transfer. Therefore, the maximum yield of $\mathbf{M e P T Z}^{\mathbf{}}$ would be obtained when the initial ratio of $\mathbf{4}$ to $\mathbf{Q}$ represents the binding stoichiometry between these two components. Using UV-vis spectrophotometry, we confirmed that these reactions were complete, prior to construction of the Job plot (Figure 2.68).

This analysis clearly shows that the reaction stoichiometry between $\mathbf{4}$ and $\mathbf{Q}$ is 1:1, which corroborates the result we obtained electrochemically indicating that $\mathbf{4}$ promotes electron transfer via a CE pathway. These data provide further evidence in support of our conclusions that HBDcoupled electron transfer requires dicationic HBDs, which complex to $\mathbf{Q}^{\mathbf{}}$ with 1:1 stoichiometry, analogous to the 2:1 complexes observed when monocationic HBDs are used.

${ }^{35}$ Rosokha, S. V.; Kochi, J. K. J. Am. Chem. Soc. 2007, 129, 3683-3697. 

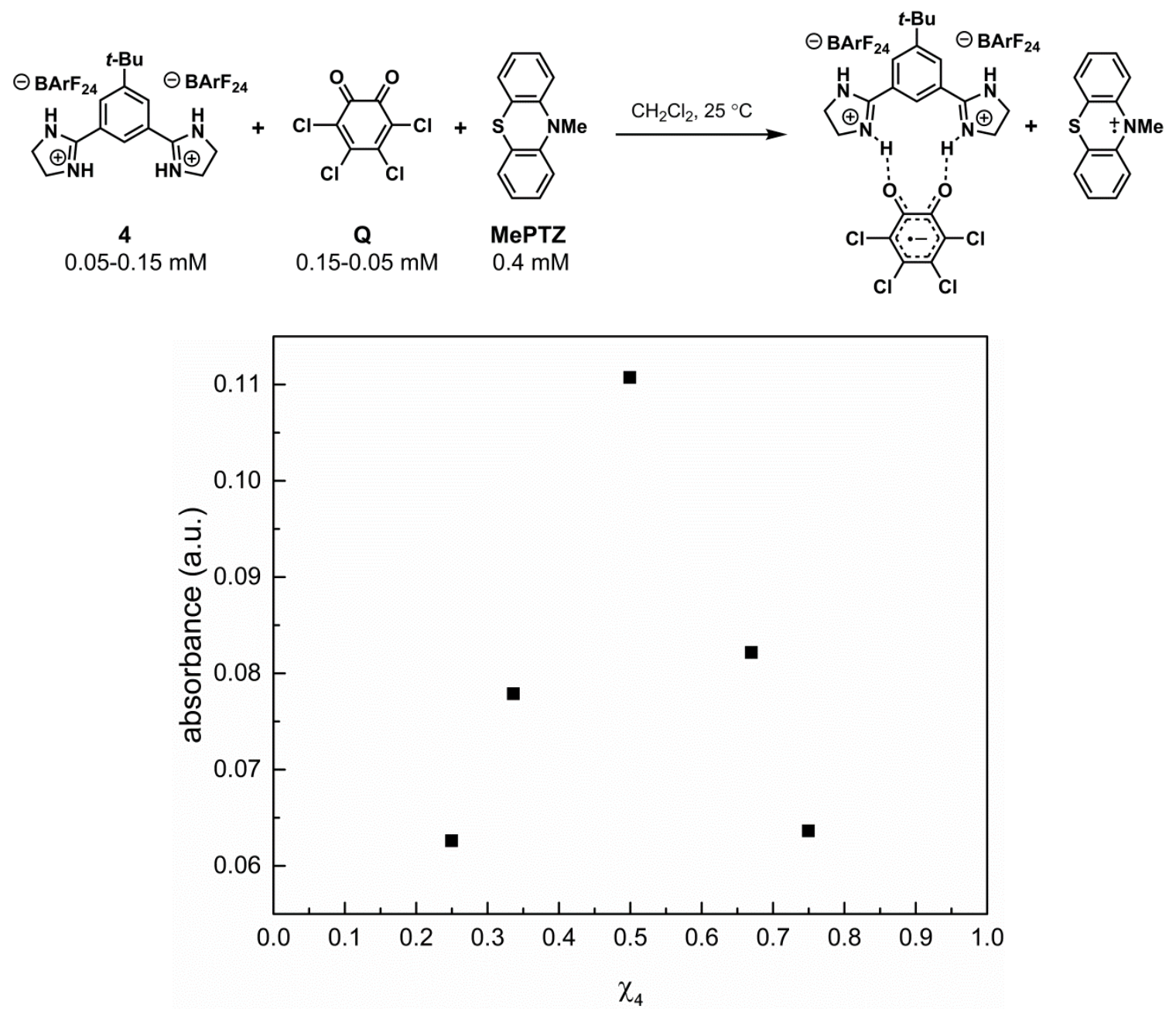

Figure 2.68. Job plot using absorbance of $\mathbf{M e P T Z}^{++}$obtained after reaction between MePTZ $(0.4 \mathrm{mM})$ and $\mathbf{Q}$ in the presence of $\mathbf{4}$ in $\mathrm{CH}_{2} \mathrm{Cl}_{2}$ at $25^{\circ} \mathrm{C}$ under $\mathrm{N}_{2}$. The total concentration of [Q] + [4] was held at $0.2 \mathrm{mM}$. [Note: the y-axis is scaled and does not start at 0].

\section{2b.5 Relative rate constants for HBD-coupled electron transfer}

To probe the effects of the HBDs on the rate of electron transfer, we determined the relative rate constants for the HBD-mediated reaction between $\mathbf{Q}$ and ferrocene derivatives. The rate constants were obtained under pseudo-first-order conditions, in which the excess of HBD was varied. Two electron donors were studied with each HBD, and the relative rates were scaled according to the intrinsic reactivity differences of those electron donors. 
A comparison of the relative rate constants with the corresponding equilibrium constants reveals a strong correlation between the thermodynamics and kinetics of electron transfer (Table 2.15). It is evident that H-bonding and Coulombic attraction both play an important role in lowering the barrier to electron transfer, in the same way as they stabilize the ground state by binding to $\mathbf{Q}^{\bullet-}$. Acidity, as a surrogate for H-bonding ability, has a clear effect on the kinetics of electron transfer, This is indicated by the relationship between the relative rates observed with $\mathbf{1}$, 2, and 8, which show an improvement in rate enhancement as HBD acidity increases. For example, HBDs $\mathbf{2}$ and $\mathbf{8}$ are both more acidic than 1, and also exhibit a greater degree of rate acceleration. Electrostatic interactions are also critical, evidenced by the minimal rate acceleration afforded by neutral 3. The negligible difference in rate afforded by $\mathbf{1}$ relative to 7 demonstrates that constraining the conformation of the guanidinium has no impact on the kinetics of electron transfer. This is consistent with our electrochemical observation that conformational rigidity also has little discernible effect on binding to $\mathbf{Q}^{\bullet-}$. 
Table 2.15. Relative rate constants for HBD-coupled electron transfer. ${ }^{\text {a }}$

\begin{tabular}{|c|c|c|c|c|c|c|}
\hline & Q & RFc & & & & \\
\hline HBD & $\begin{array}{c}\mathrm{k}_{\mathrm{obs}}\left(\mathrm{s}^{-1}\right) \\
(\mathbf{F c})\end{array}$ & $\begin{array}{c}\mathrm{k}_{\mathrm{rel}}\left(\mathrm{s}^{-1}\right) \\
(\mathbf{B r F c})\end{array}$ & $\begin{array}{l}\mathrm{k}_{\mathrm{rel}}\left(\mathrm{s}^{-1}\right) \\
\left(\mathbf{B r}_{2} \mathbf{F c}\right)\end{array}$ & $\mathrm{k}_{\mathrm{rel}}\left(\mathrm{s}^{-1}\right)^{\mathrm{b}}$ & $\mathrm{K}_{3}\left(\mathrm{M}^{-1}\right)$ & $\mathrm{K}_{3} \mathrm{~K}_{4}\left(\mathrm{M}^{-2}\right)$ \\
\hline 3 & 1 & -- & -- & 1 & $5.6 \times 10^{4}$ & $1.0 \times 10^{7}$ \\
\hline 7 & 355 & -- & -- & $3.6 \times 10^{2}$ & $3.2 \times 10^{5}$ & $2.2 \times 10^{8}$ \\
\hline 1 & 486 & 1 & -- & $4.9 \times 10^{2}$ & $3.4 \times 10^{4}$ & $6.1 \times 10^{8}$ \\
\hline 8 & -- & 2.75 & -- & $5.2 \times 10^{5}$ & $3.5 \times 10^{4}$ & $2.9 \times 10^{9}$ \\
\hline 2 & -- & 124 & 1 & $2.3 \times 10^{7}$ & $3.5 \times 10^{5}$ & $1.8 \times 10^{10}$ \\
\hline 4 & -- & -- & 104 & $9.0 \times 10^{11}$ & $9.2 \times 10^{10}$ & -- \\
\hline
\end{tabular}

${ }^{a}$ Pseudo-first-order rate constants were determined at $25^{\circ} \mathrm{C}$ by monitoring the reaction between Q $(2.5 \mathrm{mM})$ and the indicated ferrocene derivative $(0.5 \mathrm{mM})$ in $\mathrm{CH}_{2} \mathrm{Cl}_{2}$ in the presence of the indicated HBD $(5.0 \mathrm{mM})$. The unscaled relative rate constants are shown on the left. ${ }^{\mathrm{b}}$ All units for relative rate constants are assumed to be identical. ${ }^{36}$

Bis-amidinium salt 4 provides remarkable acceleration of the rate of electron transfer, with a relative rate constant that is 12 orders of magnitude larger than that for urea $\mathbf{3}$. Even when compared with $\mathbf{2}$ the rate enhancement is substantial, as $\mathbf{4}$ affords rate acceleration five orders of magnitude greater. Combined with the low rate enhancement provided by $\mathbf{3}$, the potency of $\mathbf{4}$ reinforces our proposed importance of electrostatics in promoting electron transfer. It further

\footnotetext{
${ }^{36}$ Although the different HBDs display different kinetic orders, a large excess of HBD was used under the pseudo-first-order conditions. Therefore, the concentration of HBD is assumed to be constant over the course of the electron transfer, and does not affect the units of the relative rate constants.
} 
illustrates that dicationic HBDs are superior in their ability to participate in HBD-coupled electron transfers.

\section{2b.6 Large rate acceleration provided by diphenylformamidinium salts}

When $\mathbf{9}$ is employed to mediate the reaction between $\mathbf{Q}$ and $\mathbf{B r}_{2} \mathbf{F c}$, electron transfer occurs rapidly and essentially reaches completion within 20 seconds (Figure 2.69). Because electron transfer is so rapid, a quantitative value for $\mathrm{k}_{\mathrm{rel}}$ could not be obtained using UV-vis techniques. This rapid reaction rate could be consistent with protonation of $\mathbf{Q}$ by $\mathbf{9}$, which would also explain the irreversibility observed in the electrochemical titration with this HBD. However, determining the origin of this large rate enhancement requires further investigation. A kinetic isotope effect experiment, using deuterated 9 at the H-bonding site, may allow for discrimination between the H-bonding and protonation pathways. Nonetheless, this large effect provides a compelling basis for future catalyst development, which motivates our presentation of this remarkable, albeit qualitative effect, here. 

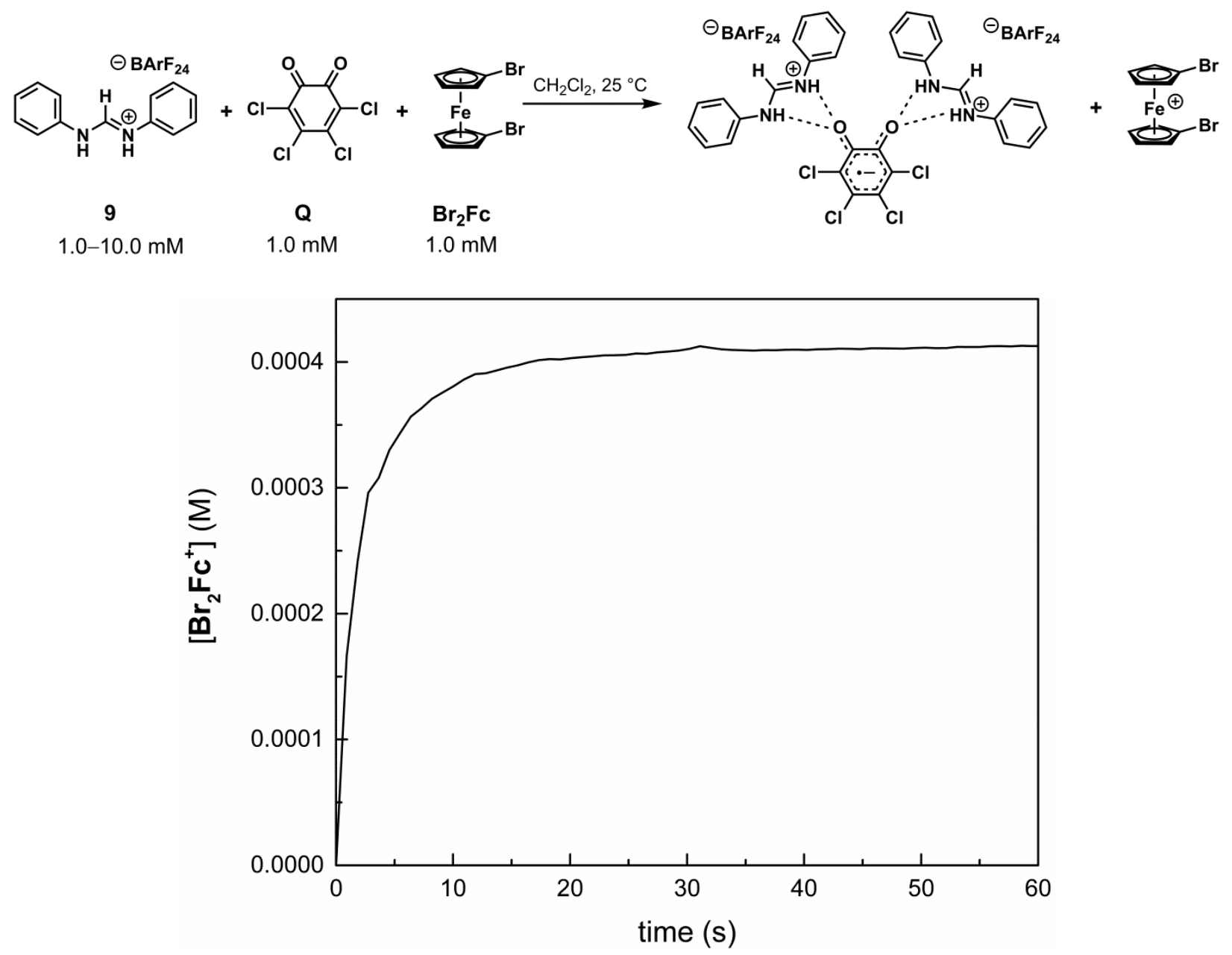

Figure 2.69. Raw data for electron transfer from $\mathbf{B r}_{2} \mathbf{F c}(0.5 \mathrm{mM})$ to $o$-chloranil $(2.5 \mathrm{mM})$ promoted by $9(5.0 \mathrm{mM})$, in $\mathrm{CH}_{2} \mathrm{Cl}_{2}$ at $25^{\circ} \mathrm{C}$.

\section{2b.7 Thermodynamic analysis of homogeneous electron transfer processes}

By studying these homogeneous electron transfers kinetically, we also gain a new perspective on the degree to which 4 modulates the thermodynamics of electron transfer. Electron transfer between 1,1'-dibromoferrocene and $\mathbf{Q}$ is highly unfavorable in the absence of an $\operatorname{HBD}\left(\Delta \mathrm{G}_{\mathrm{ET}}=+15.3 \mathrm{kcal} / \mathrm{mol}\right)$, yet 4 modulates the kinetics and thermodynamics of this inherently disfavored process such that it proceeds rapidly. In comparison, DDQ $\left(\mathrm{E}_{1 / 2}=0.12 \mathrm{~V}\right.$ 
vs. $\left.\mathrm{Fc}^{+/ 0}\right),{ }^{37}$ a commonly used and more powerful oxidant than $\mathbf{Q}\left(\mathrm{E}_{1 / 2}=-0.35 \mathrm{~V}\right.$ vs. $\left.\mathrm{Fc}^{+/ 0}\right)$, is not intrinsically reactive enough to independently perform this electron transfer reaction $\left(\Delta \mathrm{G}_{\mathrm{ET}}=\right.$ $+4.4 \mathrm{kcal} / \mathrm{mol})$. The generality regarding the large effect of 4 in HBD-coupled electron transfer reactions was further examined with additional electron donors, revealing that $\mathbf{4}$ facilitates oxidation of perylene in a yet more unfavorable process $\left(\Delta \mathrm{G}_{\mathrm{ET}}=+19.8 \mathrm{kcal} / \mathrm{mol}\right)^{38}$ (Figure 2.70$)$. These experiments demonstrate that $\mathbf{4}$ allows for successful implementation HBD-coupled electron transfer with electron-deficient quinones, by modulating both the thermodynamics and kinetics of intrinsically disfavored electron transfer reactions. The fact that $\mathbf{4}$ can surmount such large barriers to reactivity further illustrates its promise for use in synthetically relevant redox events requiring strong oxidants.

${ }^{37}$ Connelly, N. G.; Geiger, W. E. Chem. Rev. 1996, 96, 877-910.

${ }^{38}$ Cui, X.; Charaf-Eddin, A.; Wang, J.; Le Guennic, B.; Zhao, J.; Jacquemin, D. J. Org. Chem. 2014, 79, 2038-2048. 

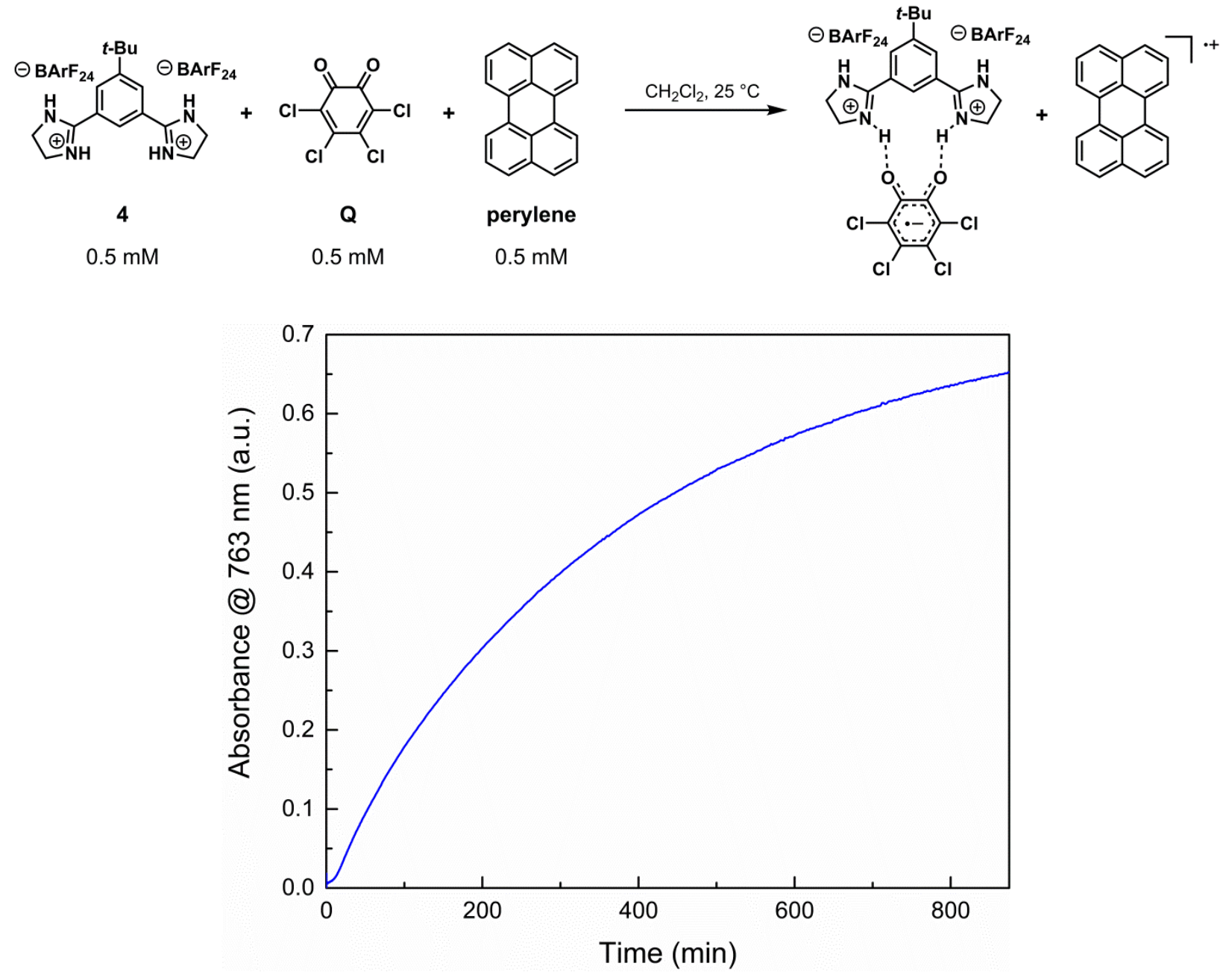

Figure 2.70. Raw data for electron transfer from perylene $(0.5 \mathrm{mM})$ to $o$-chloranil $(0.5 \mathrm{mM})$ in $\mathrm{CH}_{2} \mathrm{Cl}_{2}$ at $25^{\circ} \mathrm{C}$ promoted by $4(0.5 \mathrm{mM})$. Absorbance of perylene ${ }^{\circ+}\left(\lambda_{\max }=763 \mathrm{~nm}\right)$ vs. time.

\section{2b.8 Experimental details}

\section{2b.8.1 General information}

Commercial reagents were purchased from Sigma-Aldrich, Alfa Aesar, Acros, Strem Chemicals, and Matrix Scientific, and were used as received unless otherwise noted. Dichloromethane, N,N-dimethylformamide, diethyl ether, tert-butyl methyl ether, and tetrahydrofuran were purified by passing through a column of activated alumina. 


\section{General information for kinetics measurements}

Dichloromethane, which was purified according to known literature procedures, ${ }^{39}$ was subjected to three freeze-pump-thaw cycles prior to use and was used within four days of degassing. Ferrocene was purified by recrystallization from cyclohexane. Bromoferrocene was purified by passing through an alumina plug with hexanes. Dibromoferrocene was recrystallized from methanol at $-78{ }^{\circ} \mathrm{C}$ and was dried in vacuo after filtration. $N$-Methylphenothiazine was recrystallized from ethanol. All glassware was oven-dried prior to use. Cuvettes were stored in a desiccator over Drierite and $\mathrm{P}_{2} \mathrm{O}_{5}$. All stock solutions were prepared in volumetric flasks with septum-seal screw caps under a nitrogen atmosphere. Solvents and solutions were transferred by stainless-steel syringes using standard inert atmosphere techniques. Samples were studied in quartz cuvettes (Starna) with 1-cm path length, fitted with a septum-seal screw cap. The UV-vis experiments were performed using an Agilent Cary 60 spectrophotometer equipped with a Quantum Northwest T2 temperature regulator. All kinetics data, data for Job plots, and data for Beer's Law plots were acquired at $25{ }^{\circ} \mathrm{C}$. The increase in absorbance at the $\lambda_{\max }$ of the appropriate cationic product species was monitored over the course of the reaction and was used to evaluate the kinetic profile of each reaction. The concentration vs. time data were empirically fit to a ninth-order polynomial $\left([\right.$ product $]=\mathrm{f}(\mathrm{t})=\mathrm{at}^{9}+\mathrm{bt}^{8}+\mathrm{ct}^{7}+\mathrm{dt}^{6}+\mathrm{et}^{5}+\mathrm{ft}^{4}+\mathrm{gt}^{3}+\mathrm{ht}^{2}+\mathrm{it}+$ j). The derivative of this polynomial, solved at a given time, gives the corresponding observed rate constant at that time. The rate constants were thus obtained by solving the derivative for the times corresponding to the appropriate conversions. Determination of kinetic order in HBD was determined using instantaneous rates at $5 \%$ conversion, applying an initial rates assumption. This

${ }^{39}$ Armarego, W. L. F.; Chai, C. L. L. Purification of Laboratory Chemicals, $6^{\text {th }}$ ed.; Elsevier: Oxford, UK., 2009 
analysis using instantaneous rates was necessary due to a burst in reactivity that occurs up to $2-3 \%$ conversion.

Conversion of the absorbance data to concentration was achieved using values for $\varepsilon$ that were measured for $\mathrm{BrFcBArF}_{24}, \mathrm{Br}_{2} \mathrm{FcBArF}_{24}, \mathrm{FcBArF}_{24}, \mathrm{Me}_{2} \mathrm{BArF}_{24}$, and $\mathrm{MePTZBArF}_{24}$. These salts were prepared via a modification of two previously reported procedures ${ }^{40}$ : DDQ $(0.5$ equiv.), $\mathrm{NaBArF}_{24}$ (1.0 equiv.), and the appropriate electron donor (1.0 equiv.) were combined in a Schlenk flask and dissolved in $\mathrm{Et}_{2} \mathrm{O}(0.05 \mathrm{M}) . \mathrm{HCl} \cdot \mathrm{Et}_{2} \mathrm{O}$ (1.0 equiv.) was added and the reaction mixture stirred for 15 minutes before filtering under air-free conditions to remove the precipitated $\mathrm{NaCl}$. The filtrate was concentrated and dissolved in DCM, and was filtered again to remove the hydroquinone.

\section{2b.8.2 Beer's Law plots}

\section{General procedure for Beer's Law plots}

A stock solution of the appropriate ferrocenium salt in $\mathrm{CH}_{2} \mathrm{Cl}_{2}$ was prepared in a $1 \mathrm{~mL}$ volumetric flask. A quartz cuvette was charged with $1 \mathrm{~mL}$ of $\mathrm{CH}_{2} \mathrm{Cl}_{2}$. The blank solution was titrated with the ferrocenium salt, and the solution was scanned after each addition.

\footnotetext{
${ }^{40}$ (a) Khobragade, D. A.; Mahamulkar, S. G.; Pospíš̌l, L.; Císařová, I.; Rulíšek, L.; Jahn, U. Chem. Eur. J. 2012, 18, 12267-12277. (b) Manson, J. L.; Buschmann, W. E.; Miller, J. S. Inorg. Chem. 2001, 40, $1926-1935$.
} 


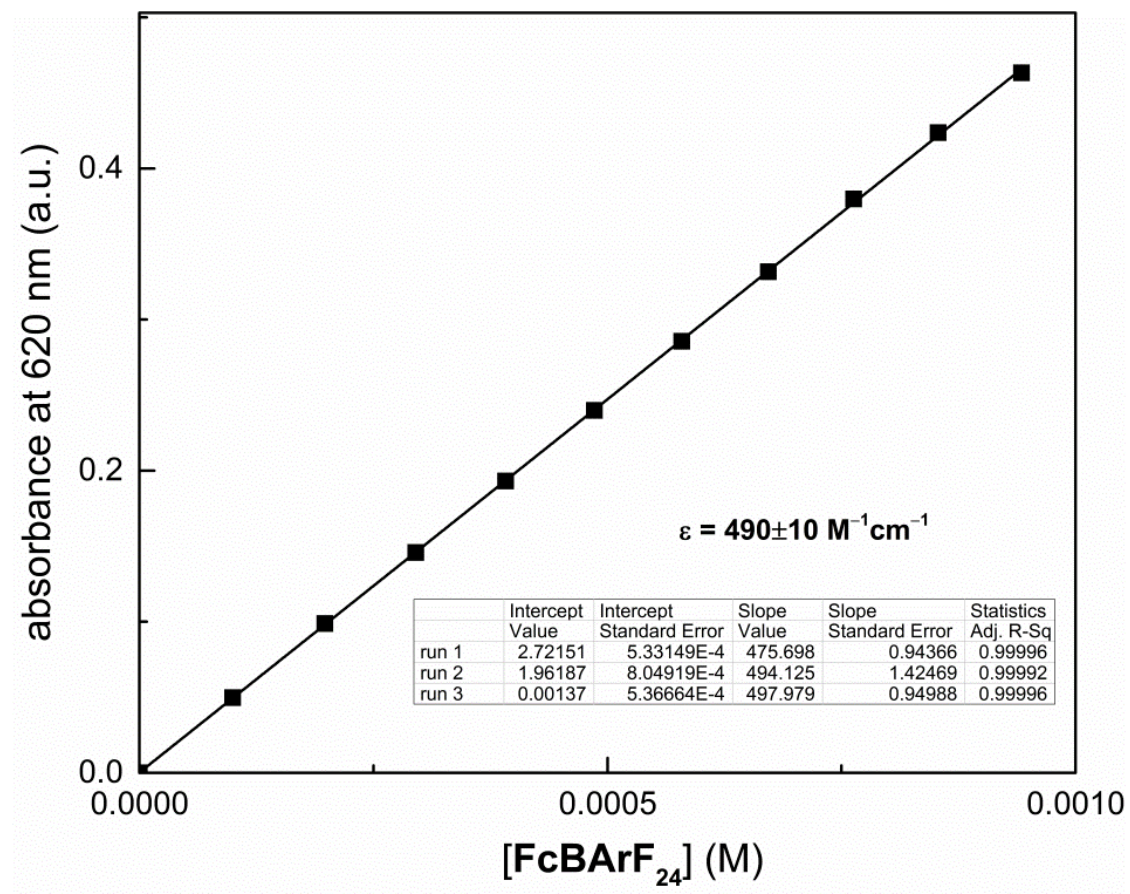

Figure 2.71. Beer's Law Plot for $\mathbf{F c B A r F}_{24}$ at $620 \mathrm{~nm}$. The average of three runs is shown.

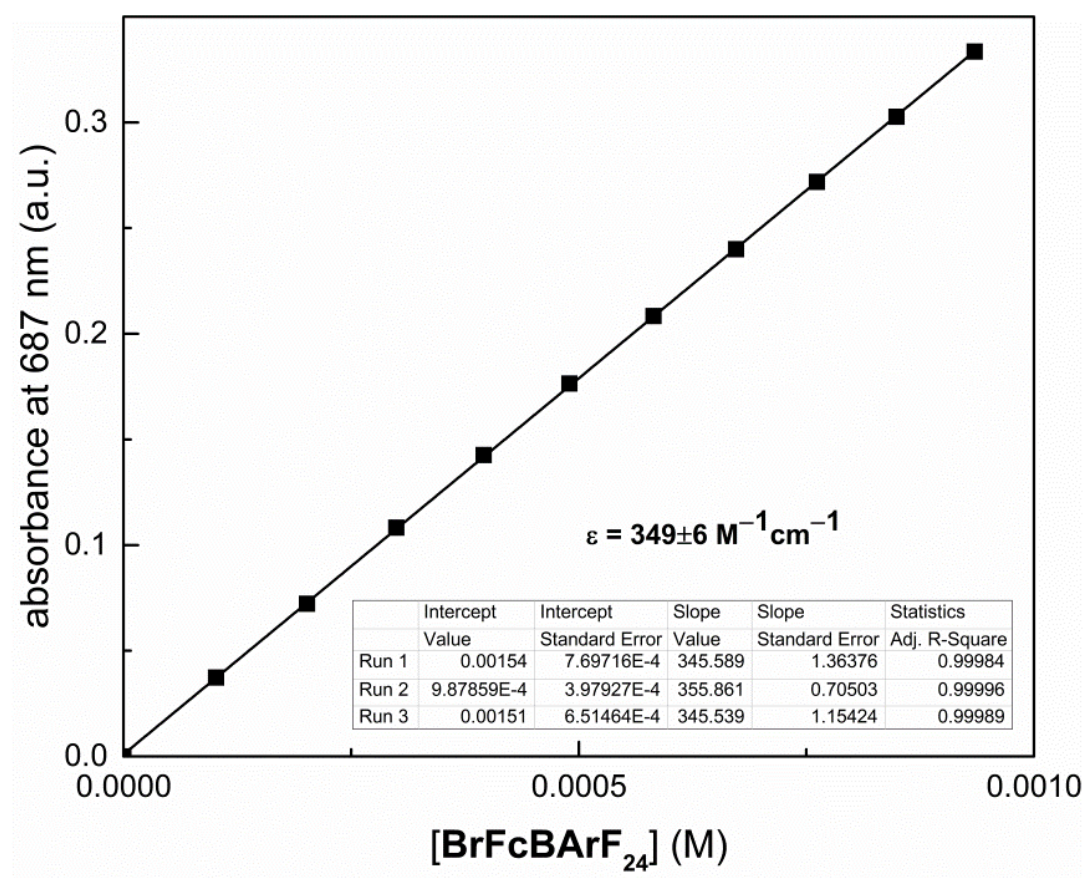

Figure 2.72. Beer's Law Plot for $\mathbf{B r F c B A r F}_{24}$ at $687 \mathrm{~nm}$. The average of three runs is shown. 


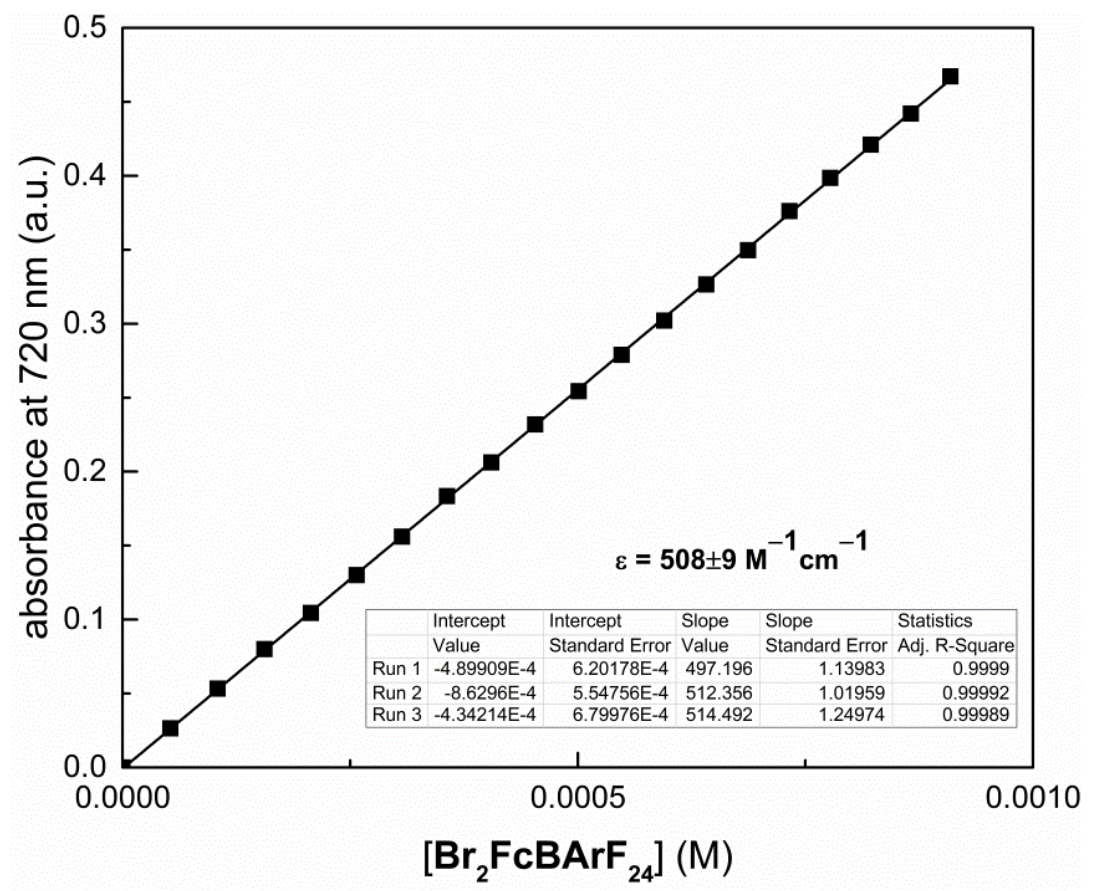

Figure 2.73. Beer's Law Plot for $\mathbf{B r}_{2} \mathbf{F c B A r F} \mathbf{F}_{24}$ at $720 \mathrm{~nm}$. The average of three runs is shown.

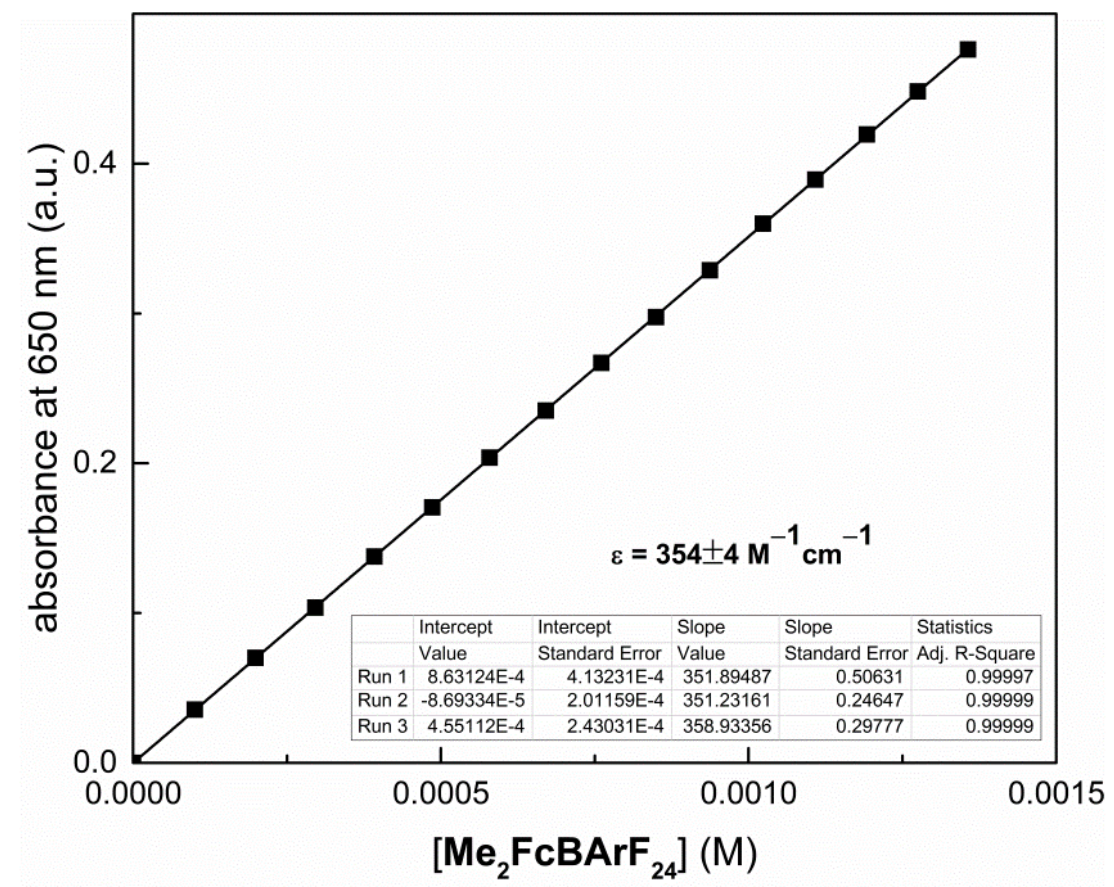

Figure 2.74. Beer's Law Plot for $\mathbf{M e}_{2} \mathbf{F c B A r F}_{24}$ at $650 \mathrm{~nm}$. The average of three runs is shown. 


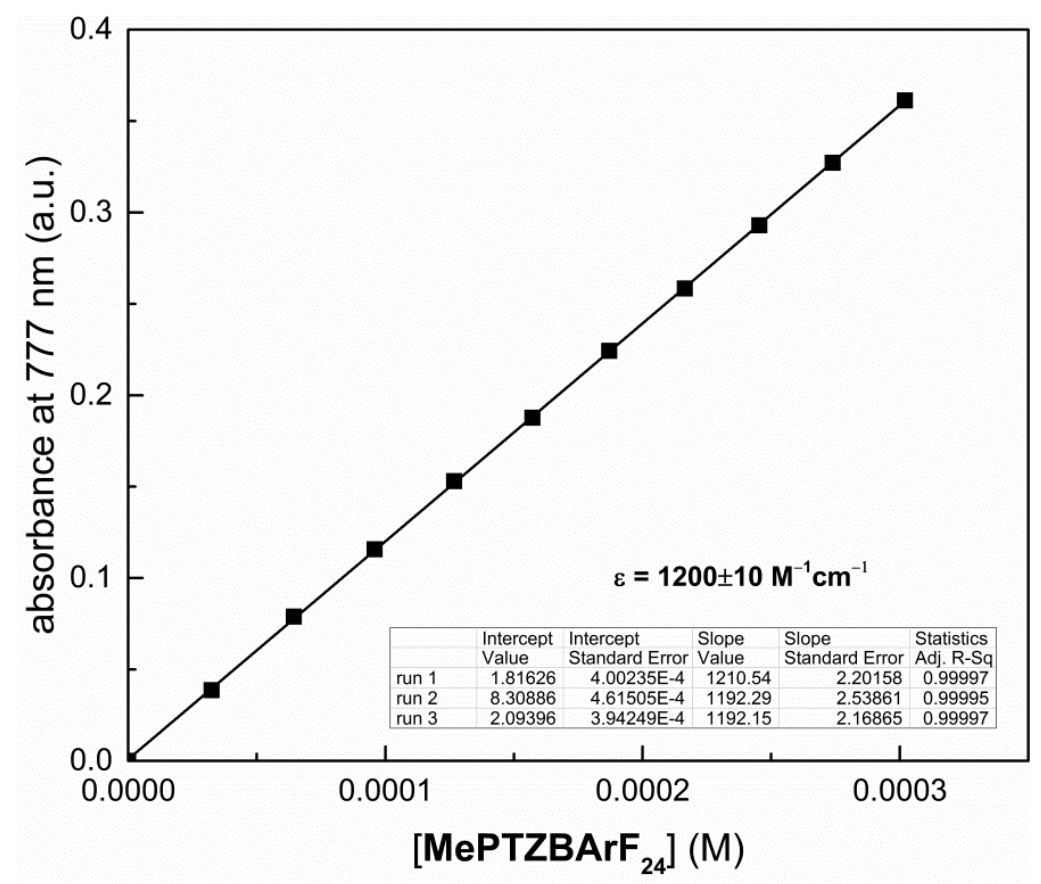

Figure 2.75. Beer's Law Plot for MePTZBArF 24 at $777 \mathrm{~nm}$. The average of three runs is shown.

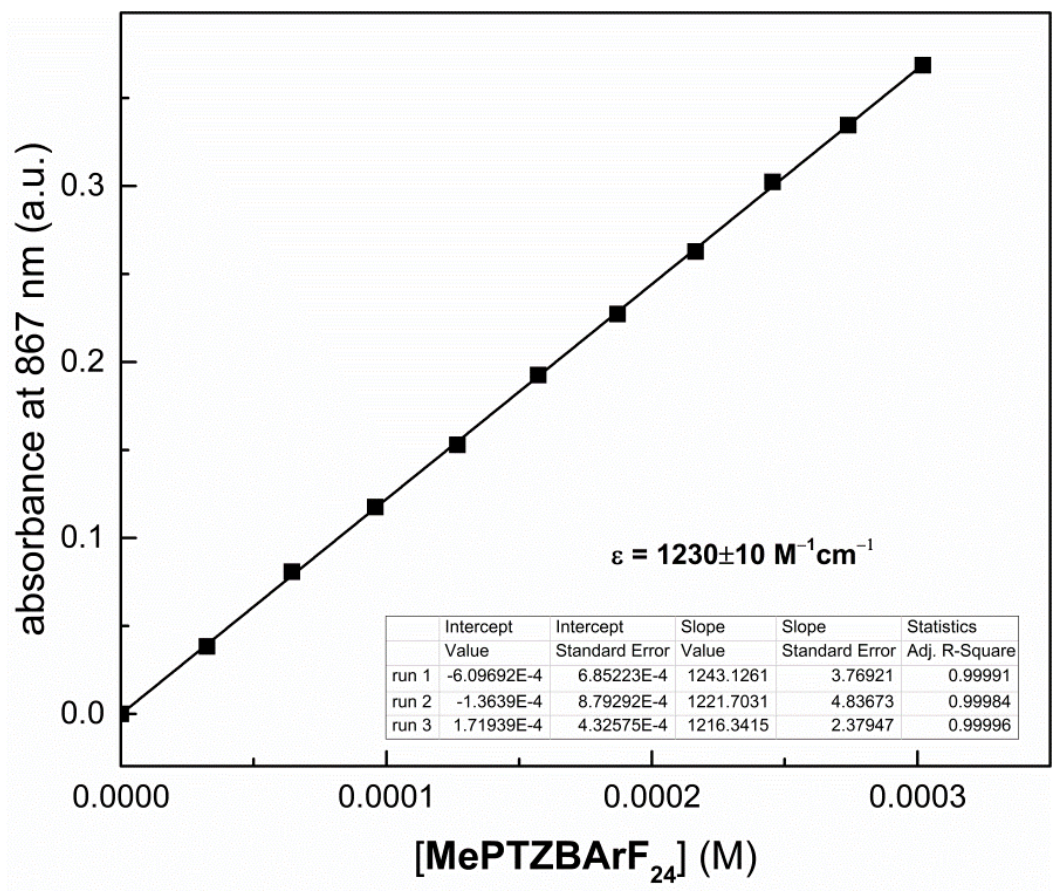

Figure 2.76. Beer's Law Plot for MePTZBArF 24 at $867 \mathrm{~nm}$. The average of three runs is shown. 


\section{2b.8.3 Initial rates experiments for determination of order in HBD}

\section{Determination of order in 1}

Stock solutions of $o$-chloranil $(0.21 \mathrm{M})$, bromoferrocene $(\mathbf{B r F c})(0.056 \mathrm{M})$, and $1(0.14$ M) were prepared in $\mathrm{CH}_{2} \mathrm{Cl}_{2}$. The appropriate amount of $\mathbf{1}$ (for six runs: $145 \mu \mathrm{L}, 130 \mu \mathrm{L}, 115 \mu \mathrm{L}$, $94 \mu \mathrm{L}, 65 \mu \mathrm{L}, 14.75 \mu \mathrm{L}$ ) and $36 \mu \mathrm{L}$ bromoferrocene were added to a $2 \mathrm{~mL}$ volumetric flask, and the solution was diluted with $\mathrm{CH}_{2} \mathrm{Cl}_{2}$. $1.5 \mathrm{~mL}$ of this solution was transferred to a cuvette equipped with a micro stir-bar under an atmosphere of nitrogen, and the cuvette was placed in the spectrophotometer. Stirring was enabled and the temperature was held at $25{ }^{\circ} \mathrm{C}$. The solution was scanned at $687 \mathrm{~nm}$ and $7 \mu \mathrm{L} o$-chloranil was added. Stirring was stopped and the reaction mixture was monitored through at least $10 \%$ conversion.

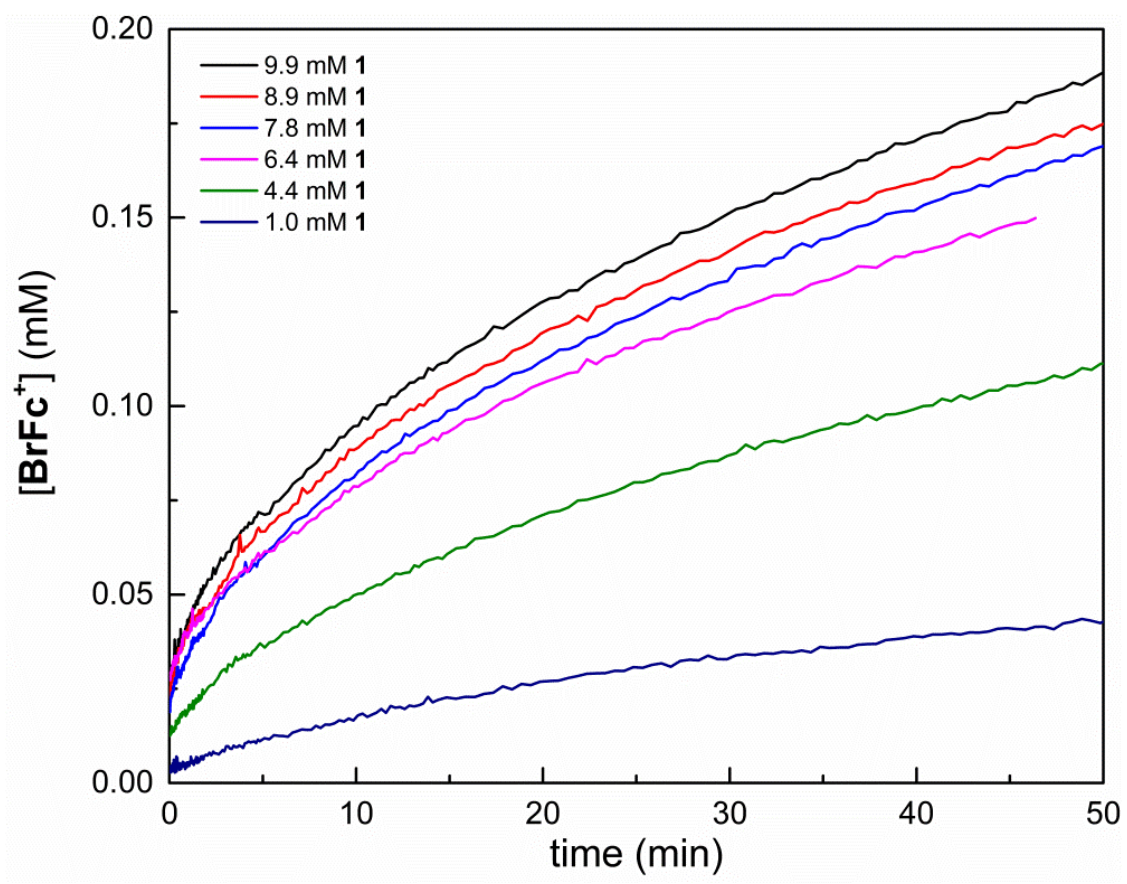

Figure 2.77. $\left[\mathrm{BrFc}^{+}\right]$vs. time for reaction of BrFc $(1.0 \mathrm{mM})$ with $o$-chloranil $(1.0 \mathrm{mM})$, with increasing [1], for two runs. 

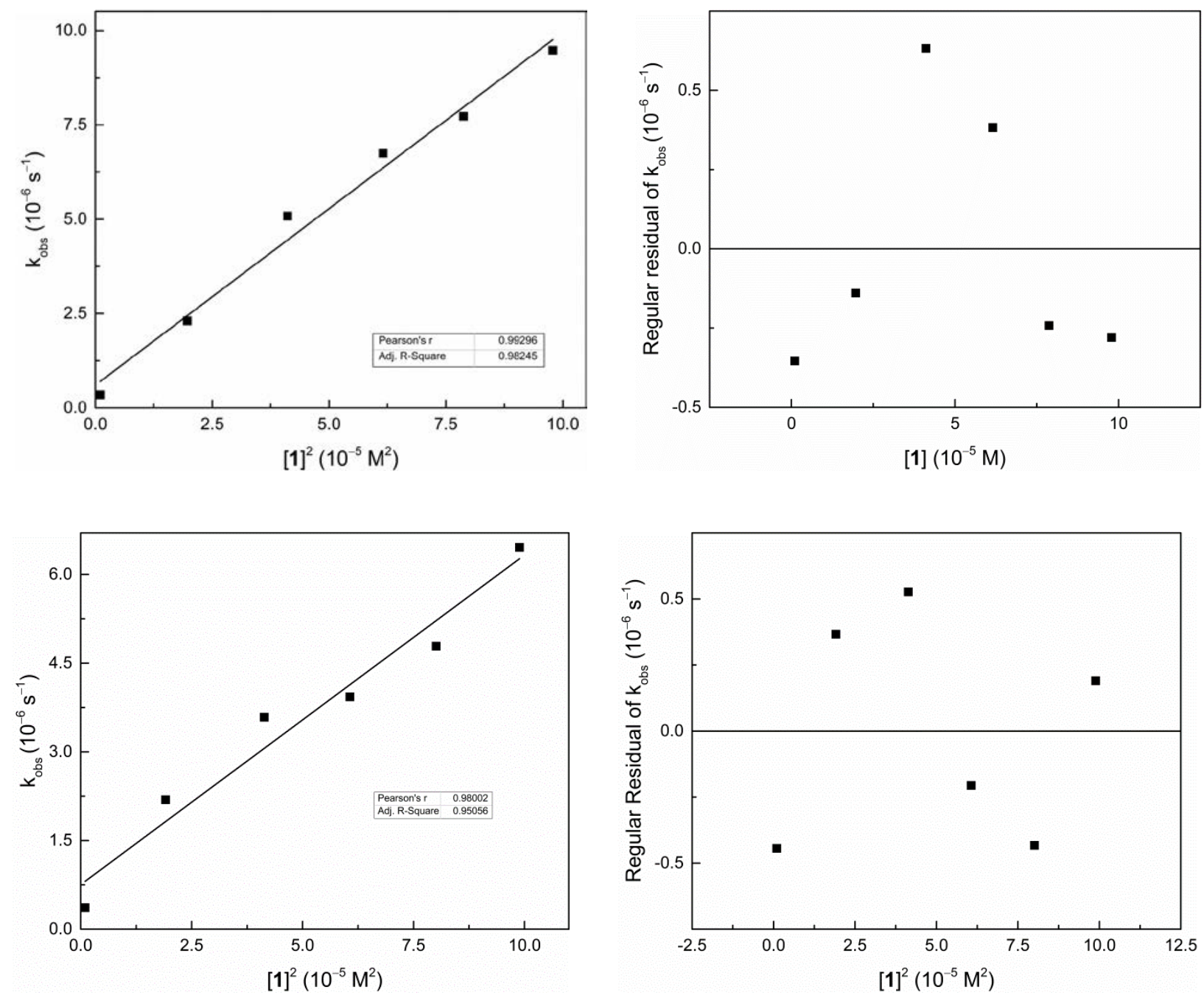

Figure 2.78. Linear fit of $\left(\mathrm{k}_{\mathrm{obs}}\right)$ vs. $[\mathbf{1}]^{2}$ and corresponding residuals for two runs.

\section{Determination of order in 2}

Stock solutions of $o$-chloranil $(0.21 \mathrm{M}), 1,1^{\prime}$-dibromoferrocene $\left(\mathbf{B r}_{2} \mathbf{F c}\right)(0.020 \mathrm{M})$, and $\mathbf{2}$ $(0.15 \mathrm{M})$ were prepared in $\mathrm{CH}_{2} \mathrm{Cl}_{2}$. The appropriate amount of 2 (for six runs: $137.5 \mu \mathrm{L}, 122.5$ $\mu \mathrm{L}, 107.5 \mu \mathrm{L}, 88 \mu \mathrm{L}, 60 \mu \mathrm{L}, 13.75 \mu \mathrm{L}$ ) and $100 \mu \mathrm{L}$ 1,1'-bromoferrocene were added to a $2 \mathrm{~mL}$ volumetric flask, and the solution was diluted with $\mathrm{CH}_{2} \mathrm{Cl}_{2} .1 .5 \mathrm{~mL}$ of this solution was transferred to a cuvette equipped with a micro stir-bar under an atmosphere of nitrogen, and the 
cuvette was placed in the spectrophotometer. Stirring was enabled and the temperature was held at $25^{\circ} \mathrm{C}$. The solution was scanned at $720 \mathrm{~nm}$ and $11.75 \mu \mathrm{L} o$-chloranil was added. Stirring was stopped and the reaction mixture was monitored through at least $10 \%$ conversion.

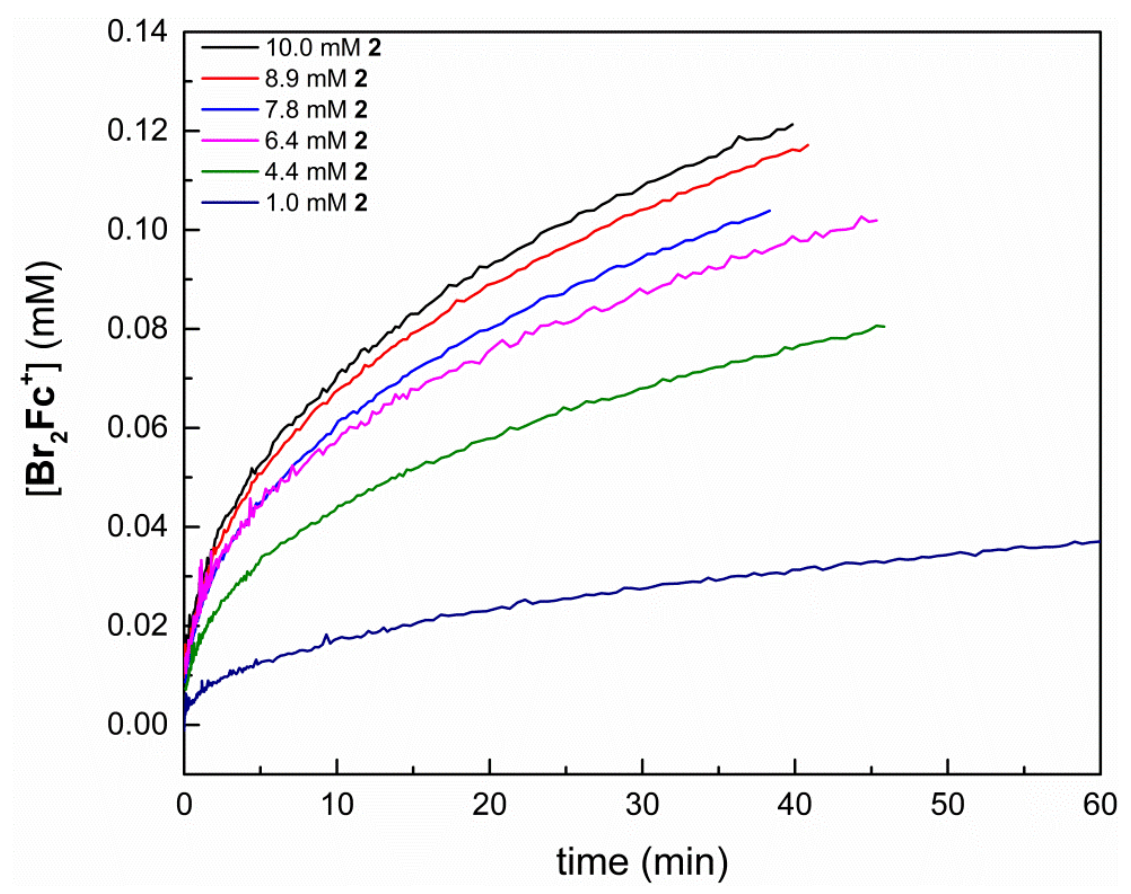

Figure 2.79. $\left[\mathrm{Br}_{2} \mathbf{F c}^{+}\right]$vs. time for reaction of $\mathbf{B r}_{2} \mathbf{F c}(1.0 \mathrm{mM})$ with $o$-chloranil $(1.0 \mathrm{mM})$ with increasing [2]. 

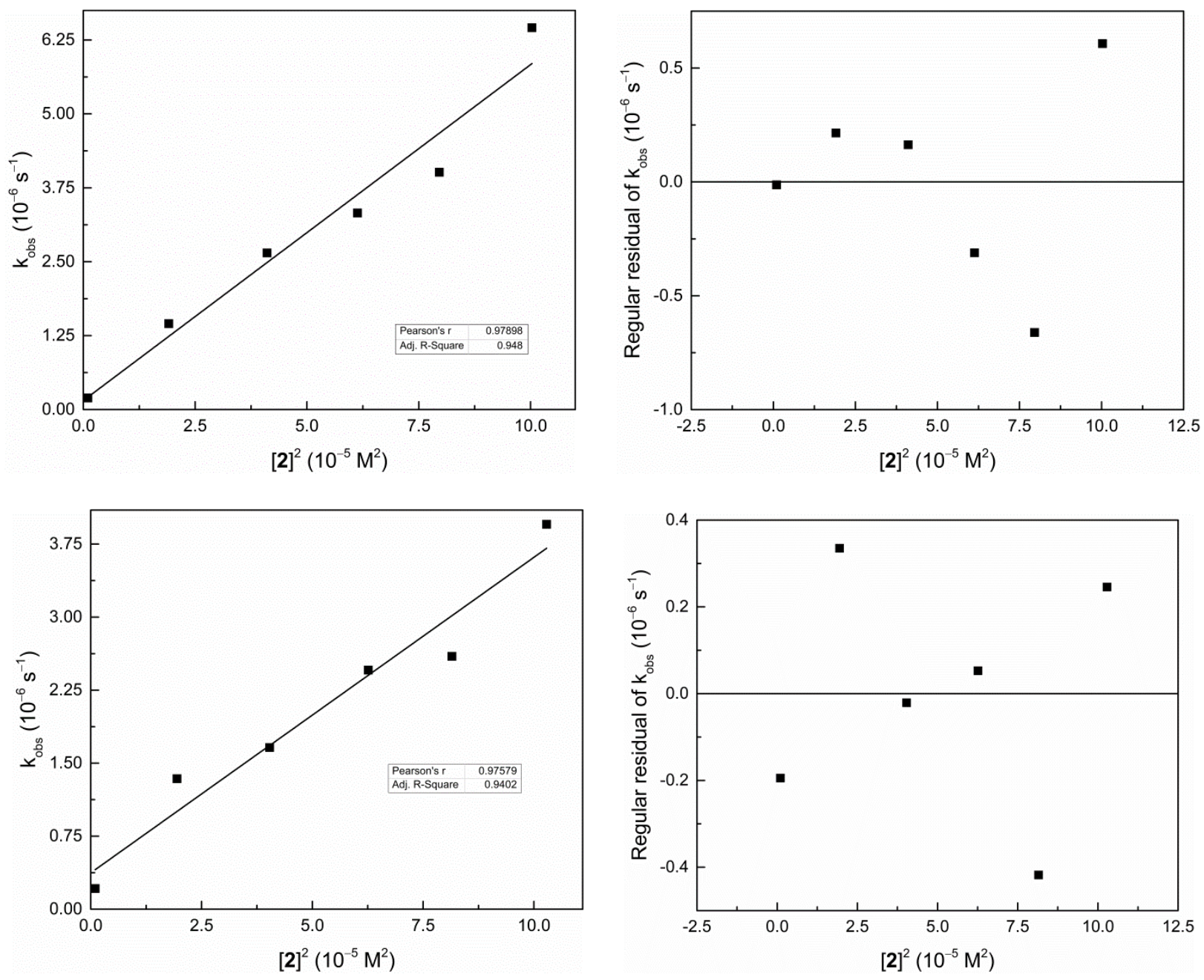

Figure 2.80. Linear fit of $\left(\mathrm{k}_{\mathrm{obs}}\right)$ vs. $[2]^{2}$ and corresponding residuals for two runs.

\section{Determination of order in 3}

Stock solutions of $o$-chloranil $(70 \mathrm{mM})$ and 1,1'-dimethylferrocene $\left(\mathbf{M e}_{2} \mathbf{F c}\right)(75 \mathrm{mM})$ were prepared in $\mathrm{CH}_{2} \mathrm{Cl}_{2}$. A stock solution of $3(52.5 \mathrm{mM})$ was prepared in $1 \mathrm{~mL} \mathrm{CH}_{2} \mathrm{Cl}_{2}$ with $200 \mu \mathrm{L}$ TBME to aid in solubilizing 3. To a $2 \mathrm{~mL}$ volumetric flask was added the appropriate amount of 3 (for six runs: $190 \mu \mathrm{L}, 157.5 \mu \mathrm{L}, 122.5 \mu \mathrm{L}, 88 \mu \mathrm{L}, 54 \mu \mathrm{L}, 19.25 \mu \mathrm{L}$ ), $13.5 \mu \mathrm{L}$ 1,1'dimethylferrocene, and TBME such that the total volume of TBME was constant across all runs $(38 \mu \mathrm{L})$. The solution was diluted with $\mathrm{CH}_{2} \mathrm{Cl}_{2} \cdot 1.5 \mathrm{~mL}$ of this solution was transferred to a 
cuvette equipped with a micro stir-bar under an atmosphere of nitrogen, and the cuvette was placed in the spectrophotometer. Stirring was enabled and the temperature was held at $25{ }^{\circ} \mathrm{C}$. The solution was scanned at $650 \mathrm{~nm}$ and $11 \mu \mathrm{L} o$-chloranil was added. Stirring was stopped and the reaction mixture was monitored through at least $10 \%$ conversion.

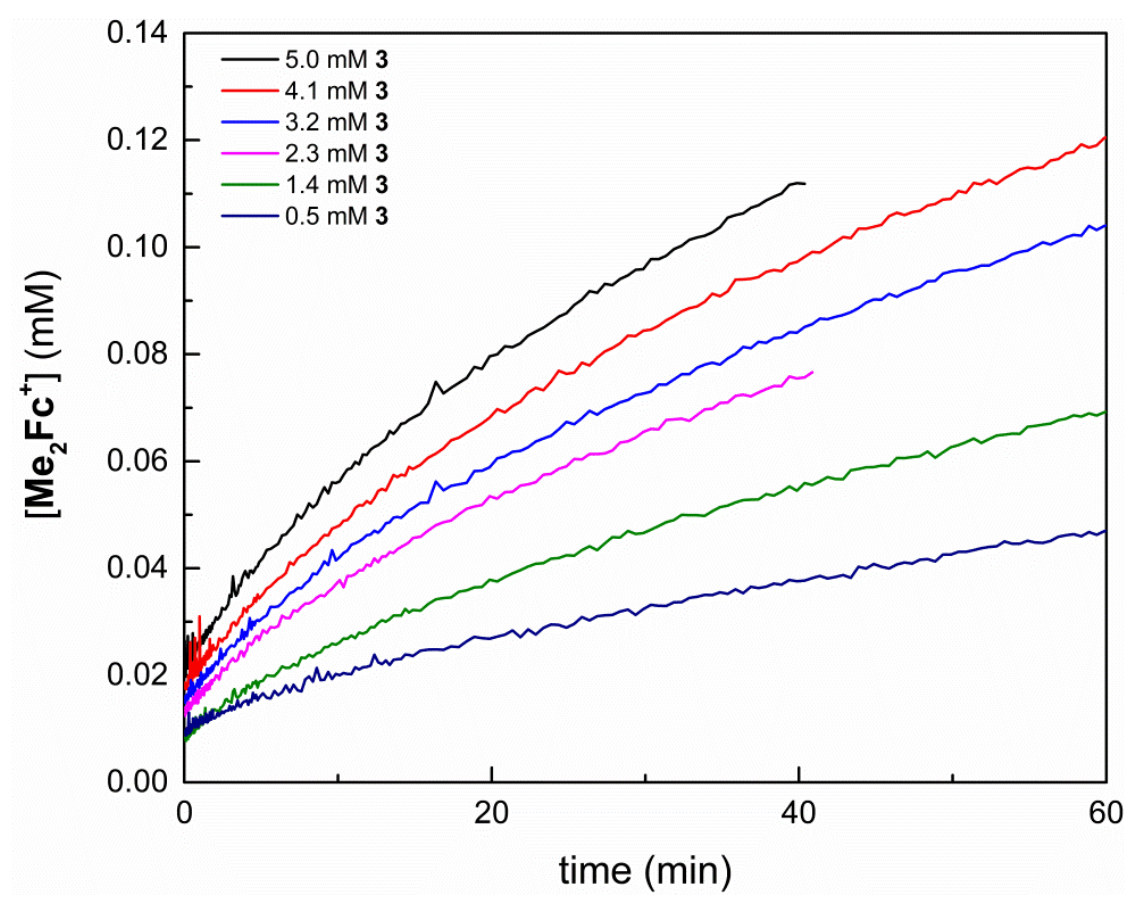

Figure 2.81. $\left[\mathrm{Me}_{2} \mathrm{Fc}^{+}\right]$vs. time for reaction of $\mathrm{Me}_{2} \mathbf{F c}(0.5 \mathrm{mM})$ with $o$-chloranil $(0.5 \mathrm{mM})$ with increasing [3]. 

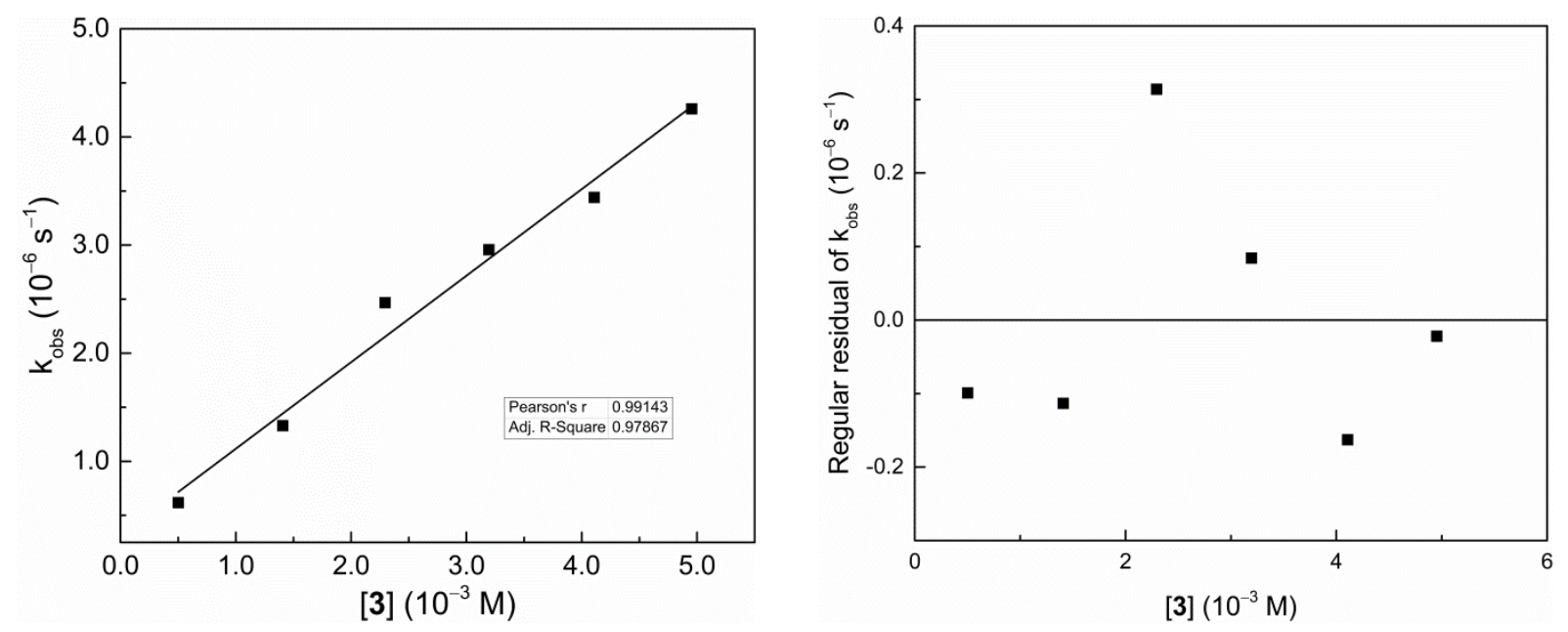

Figure 2.82. Linear fit of $\left(\mathrm{k}_{\mathrm{obs}}\right)$ vs. $[3]^{2}$ and corresponding residuals.

\section{2b.8.4 Job plot for determination of $4 \cdot Q$ stoichiometry}

Stock solutions of $o$-chloranil $(0.019 \mathrm{M})$ and $N$-methylphenothiazine (MePTZ) $(0.030 \mathrm{M})$ were prepared in $\mathrm{CH}_{2} \mathrm{Cl}_{2}$. A stock solution of $4(6.7 \mathrm{mM})$ was prepared in $2 \mathrm{~mL} \mathrm{CH}_{2} \mathrm{Cl}_{2}$. To a 2 $\mathrm{mL}$ volumetric flask were added the appropriate amounts of 4 and MePTZ $([\mathrm{MePTZ}]=0.4 \mathrm{mM})$, and the solution was diluted with $\mathrm{CH}_{2} \mathrm{Cl}_{2}$. The total concentration of $\mathbf{4}+\mathbf{Q}$ was held at $0.2 \mathrm{mM}$. $1.5 \mathrm{~mL}$ of this solution was transferred to a vial equipped with a micro stir-bar under an atmosphere of nitrogen, and $8 \mu \mathrm{L} o$-chloranil was added. The reaction was allowed to go until completion (confirmed by UV-vis) and then $1.5 \mathrm{~mL}$ of the reaction mixture was transferred to a cuvette equipped with a septum-seal screw cap. The reaction mixture was scanned through all wavelengths, and the absorbance at $\lambda_{\max }=867 \mathrm{~nm}$ was used to construct the Job plot. 


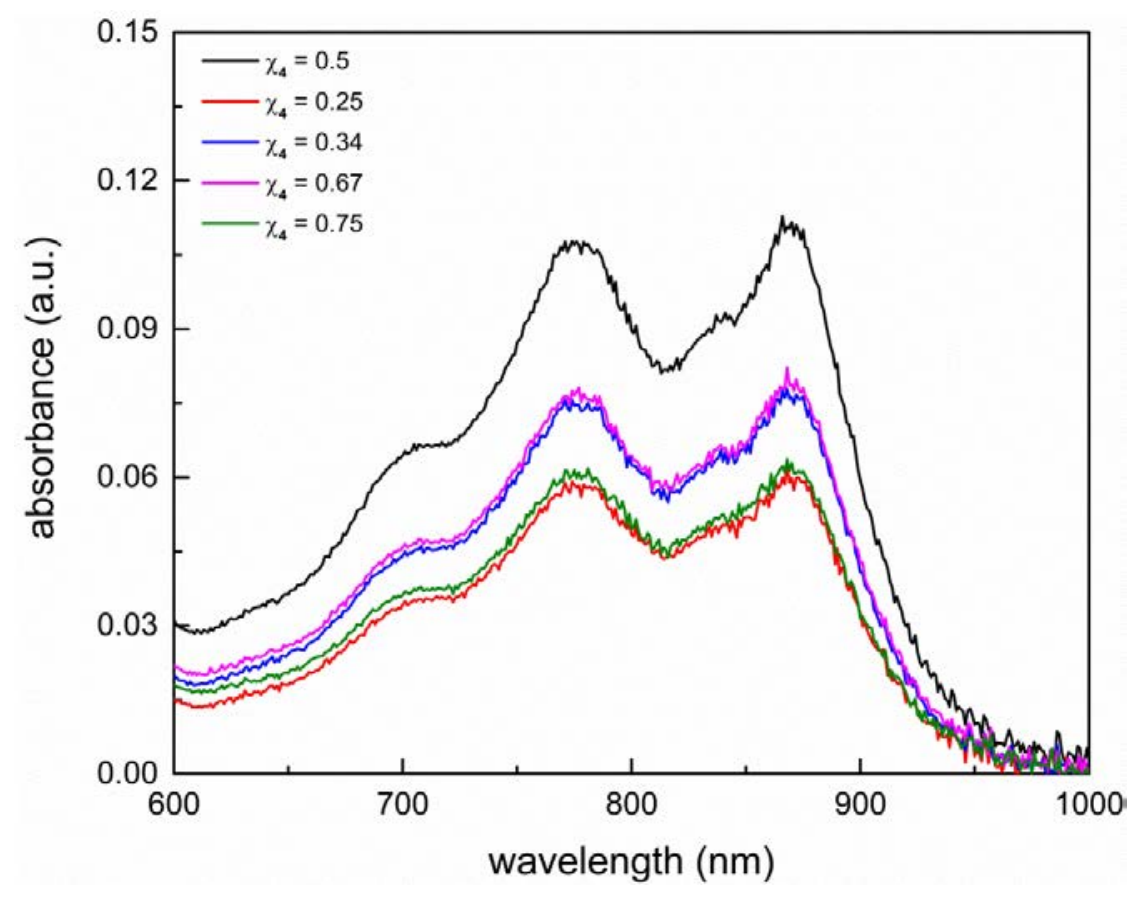

Figure 2.83. Absorbance vs. wavelength data for varying $\chi_{4}$ (with respect to $\mathbf{Q}$ ).

\section{2b.8.5 Pseudo-first-order experiments for determination of relative rate constants}

\section{General methods}

Stock solutions of $o$-chloranil, the hydrogen-bond donor, and the reductant were prepared in dichloromethane. The appropriate volumes of reductant and hydrogen-bond donor were combined in a $2 \mathrm{~mL}$ volumetric flask, and $1.5 \mathrm{~mL}$ of this solution was transferred to a cuvette, equipped with a micro stir bar. The cuvette was placed into the UV-vis spectrophotometer, and stirring was enabled. The solution was scanned at the appropriate wavelength $\left(\lambda_{\max }\right.$ of the ferrocenium ion). $o$-Chloranil was added, and stirring was stopped.

Rate constants at $50 \%$ conversion were obtained under pseudo-first-order conditions for reactions between $o$-chloranil and the appropriate ferrocene derivative, mediated by 1-4. Hydrogen-bond donors 1-3 were measured with two ferrocene derivatives each, in order to 
determine the intrinsic reactivity differences between the electron donors. The rate constants were then scaled to account for these differences.

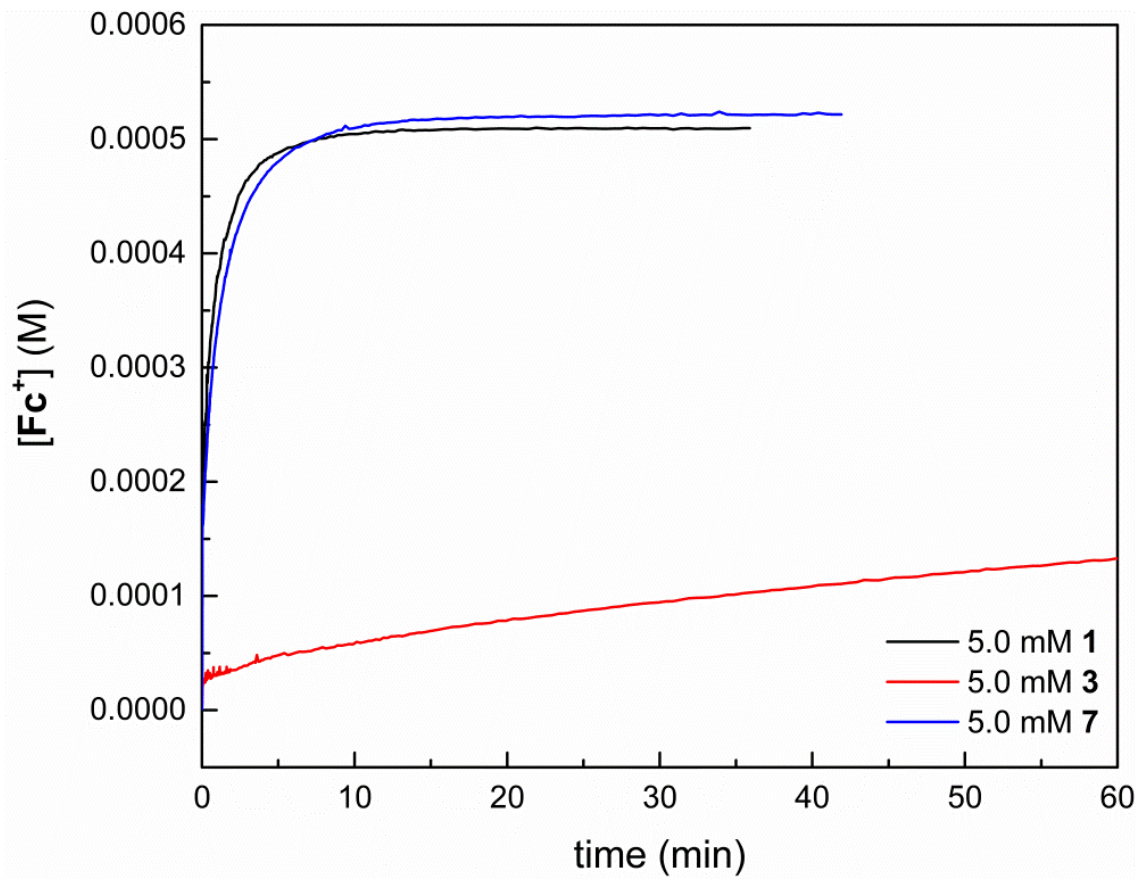

Figure 2.84. [ $\left.\mathbf{F c}^{+}\right]$vs. time for reaction of $\mathbf{F c}(0.5 \mathrm{mM})$ with $o$-chloranil $(2.5 \mathrm{mM})$, mediated by $\mathbf{1}(5.0 \mathrm{mM}), \mathbf{3}(5.0 \mathrm{mM})$, and $7(5.0 \mathrm{mM})$, in $\mathrm{CH}_{2} \mathrm{Cl}_{2}$ at $25^{\circ} \mathrm{C}$, under an atmosphere of nitrogen. 


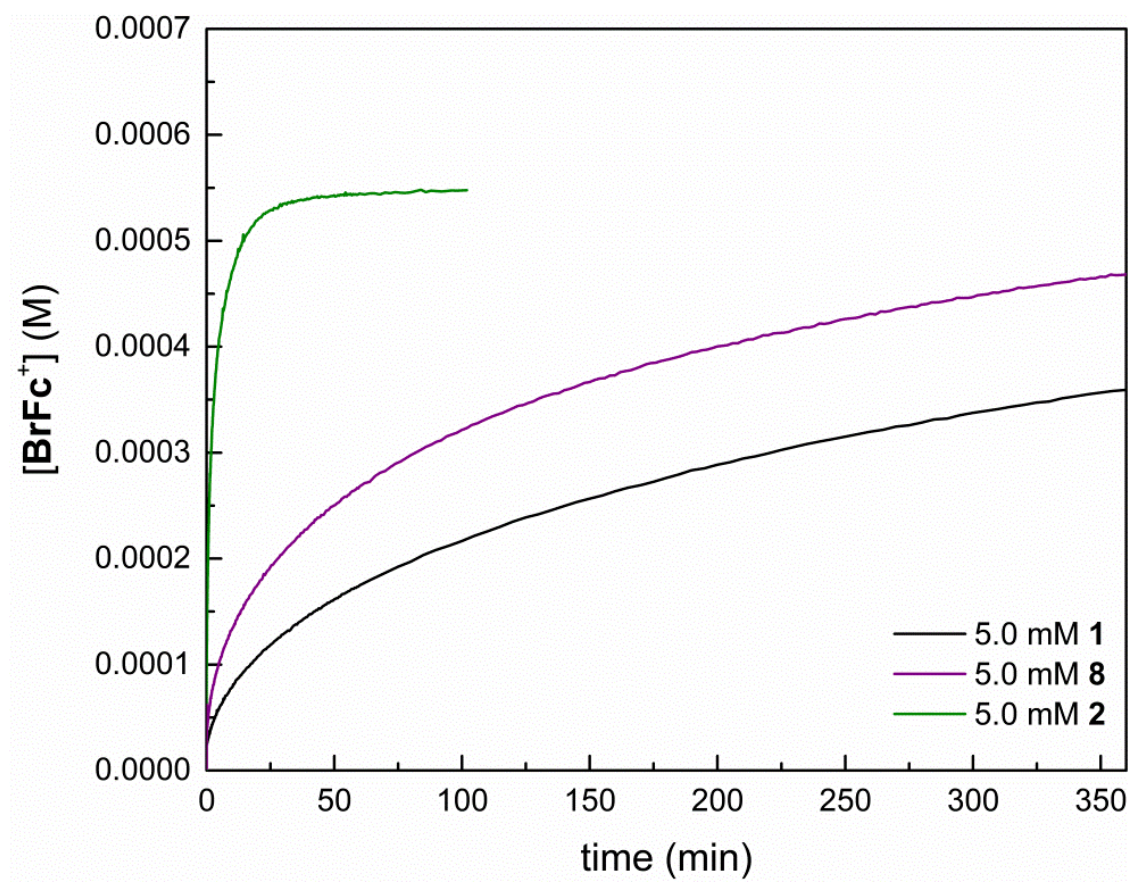

Figure 2.85. $\left[\mathbf{B r F c}^{+}\right]$vs. time for reaction of $\mathbf{B r F c}(0.5 \mathrm{mM})$ with $o$-chloranil $(2.5 \mathrm{mM})$, mediated by $1(5.0 \mathrm{mM}), 2(5.0 \mathrm{mM})$, and $\mathbf{8}(5.0 \mathrm{mM})$, in $\mathrm{CH}_{2} \mathrm{Cl}_{2}$ at $25{ }^{\circ} \mathrm{C}$, under an atmosphere of nitrogen.

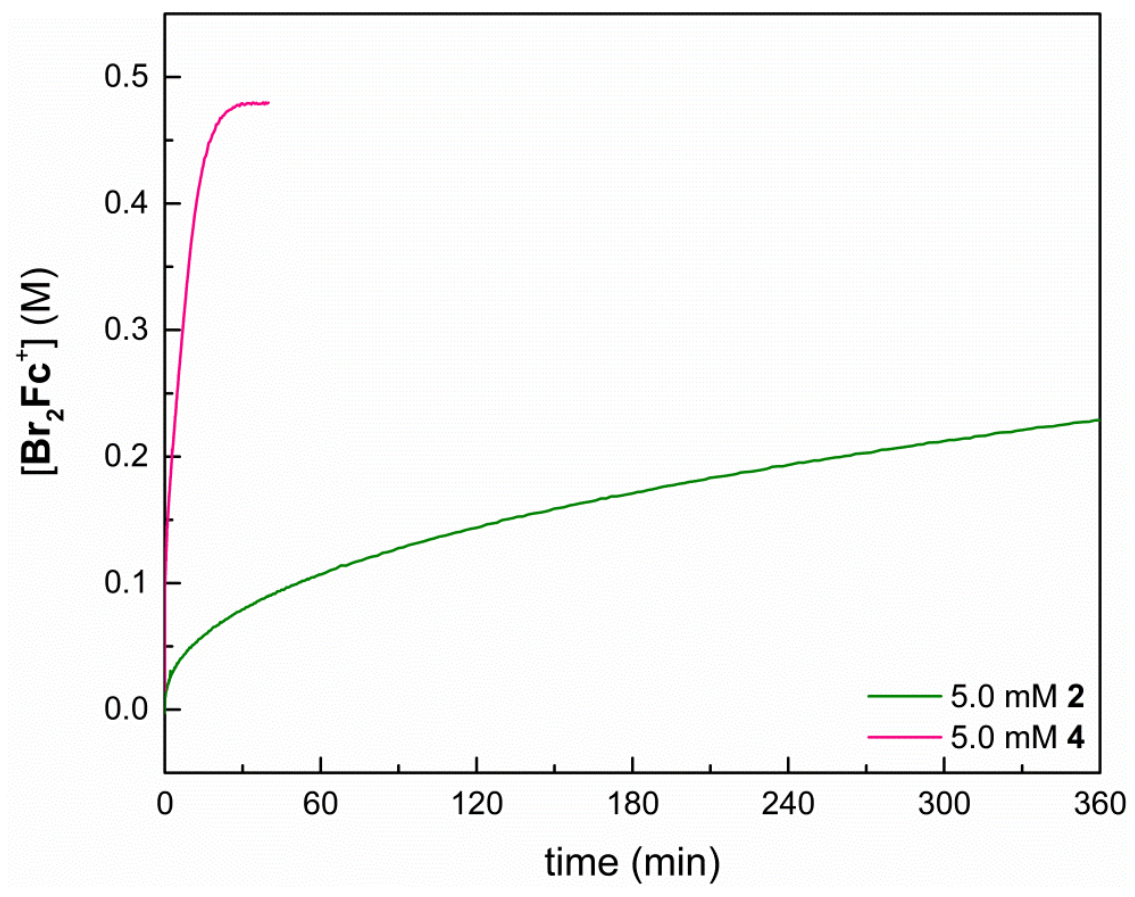

Figure 2.86. $\left[\mathrm{Br}_{2} \mathrm{Fc}^{+}\right]$vs. time for reaction of $\mathbf{B r}_{2} \mathbf{F c}(0.5 \mathrm{mM})$ with $o$-chloranil $(2.5 \mathrm{mM})$, mediated by $2(5.0 \mathrm{mM})$ and $4(5.0 \mathrm{mM})$, in $\mathrm{CH}_{2} \mathrm{Cl}_{2}$ at $25^{\circ} \mathrm{C}$, under an atmosphere of nitrogen. 
Table 2.16. Observed relative rate constants measured under pseudo-first-order conditions, before and after scaling to account for reactivity differences. The rates on the left section of the chart should be compared vertically; the rates on the right section of the chart should be compared horizontally.

\begin{tabular}{cccc|ccc|c}
\hline HBD & $\begin{array}{c}\mathbf{k}_{\text {rel }} \\
(\mathbf{w} / \mathbf{F c})\end{array}$ & $\begin{array}{c}\mathbf{k}_{\text {rel }} \\
(\mathbf{w} / \mathbf{B r F c})\end{array}$ & $\begin{array}{c}\mathbf{k}_{\text {rel }} \\
(\mathbf{w} / \mathbf{B r} \mathbf{F c})\end{array}$ & $\begin{array}{c}\mathbf{k}_{\text {rel }} \\
(\mathbf{w} / \mathbf{F c})\end{array}$ & $\begin{array}{c}\mathbf{k}_{\text {rel }} \\
(\mathbf{w} / \mathbf{B r F c})\end{array}$ & $\begin{array}{c}\mathbf{k}_{\text {rel }} \\
(\mathbf{w} / \mathbf{B r} \mathbf{F c})\end{array}$ & $\mathbf{k}_{\text {rel }}$ \\
\hline $\mathbf{3}$ & 1 & -- & -- & -- & -- & -- & compare horizontally \\
\hline $\mathbf{7}$ & 355 & -- & -- & -- & -- & -- & $3.6 \times 10^{2}$ \\
$\mathbf{1}$ & 486 & 1 & -- & 387 & 1 & -- & $4.9 \times 10^{2}$ \\
$\mathbf{8}$ & -- & 2.8 & -- & -- & -- & -- & $5.2 \times 10^{5}$ \\
$\mathbf{2}$ & -- & 124 & 1 & -- & 370 & 1 & $2.3 \times 10^{7}$ \\
$\mathbf{4}$ & -- & -- & 104 & -- & -- & -- & $9.0 \times 10^{11}$ \\
\end{tabular}

\section{Synthetic application of HBD-coupled electron transfer}

\section{2c.1 Introduction}

Having identified dicationic HBDs as particularly effective at promoting electron transfer to electron-deficient quinones, we hypothesized that bis-amidinium salts could catalyze synthetic transformations involving electron transfer. Furthermore, we speculated that the rate of such a transformation would correlate with the trends observed thermodynamically and kinetically for HBD-coupled electron transfer. To address these questions, an oxidative lactonization ${ }^{41}$ was selected as a model quinone-mediated transformation that would offer a proof-of-concept for catalysis of electron transfer using HBDs.

\footnotetext{
${ }^{41}$ Developed by Dr. David J. Hardee.
} 


\section{2c.2 Application of HBD-coupled electron transfer to a synthetic transformation}

\section{2c.2.1 Development and catalysis of an oxidative lactonization}

Quinones are known to mediate oxidative/dehydrogenative reactions that involve oxidation of benzylic and allylic ethers and alcohols. ${ }^{42}$ We envisioned that electron-rich diarylmethanes, though lacking the ether functionality, could undergo a similar oxidative transformation at the benzylic position. A putative extended oxocarbenium ion could be intercepted by a pendant nucleophile - in this case, a carboxylic acid - to afford a cyclized product.

When the various HBDs were evaluated in this oxidative lactonization, many proved to be capable of catalyzing this transformation with varying degrees of efficiency (Figure 2.87). The conversions obtained after $24 \mathrm{~h}$ reaction times were found to qualitatively correlate with both the thermodynamic and kinetic trends discussed previously (Table 2.17). Consistent with our hypothesis that HBDs with a stronger affinity for $\mathbf{Q}^{\bullet-}$ would be more effective catalysts, bisamidinium salt 4 is the most effective catalyst, affording the product in $70 \%$ yield. In comparison, neutral urea 3 does not afford any catalysis over background. The monocationic HBDs give similar yields, with a modest increase observed as binding ability improves (e.g. HBD $2>$ HBD 1$).$

\footnotetext{
${ }^{42}$ For examples, see: (a) Tu, W.; Liu, L.; Floreancig, P. E. Angew. Chem. Int. Ed. 2008, 47, 4184-4187. (b) Yu, B.; Jiang, T.; Li, J.; Su, Y.; Pan, X.; She, X. Org. Lett. 2009, 11, 3442-3445. (c) Liu, L.; Floreancig, P. E. Org. Lett. 2010, 12, 4686-4689. (d) Cosner, C. C.; Cabrera, P. J.; Byrd, K. M.; Adams Thomas, A. M.; Helquist, P. Org. Lett. 2011, 13, 2071-2073.
} 


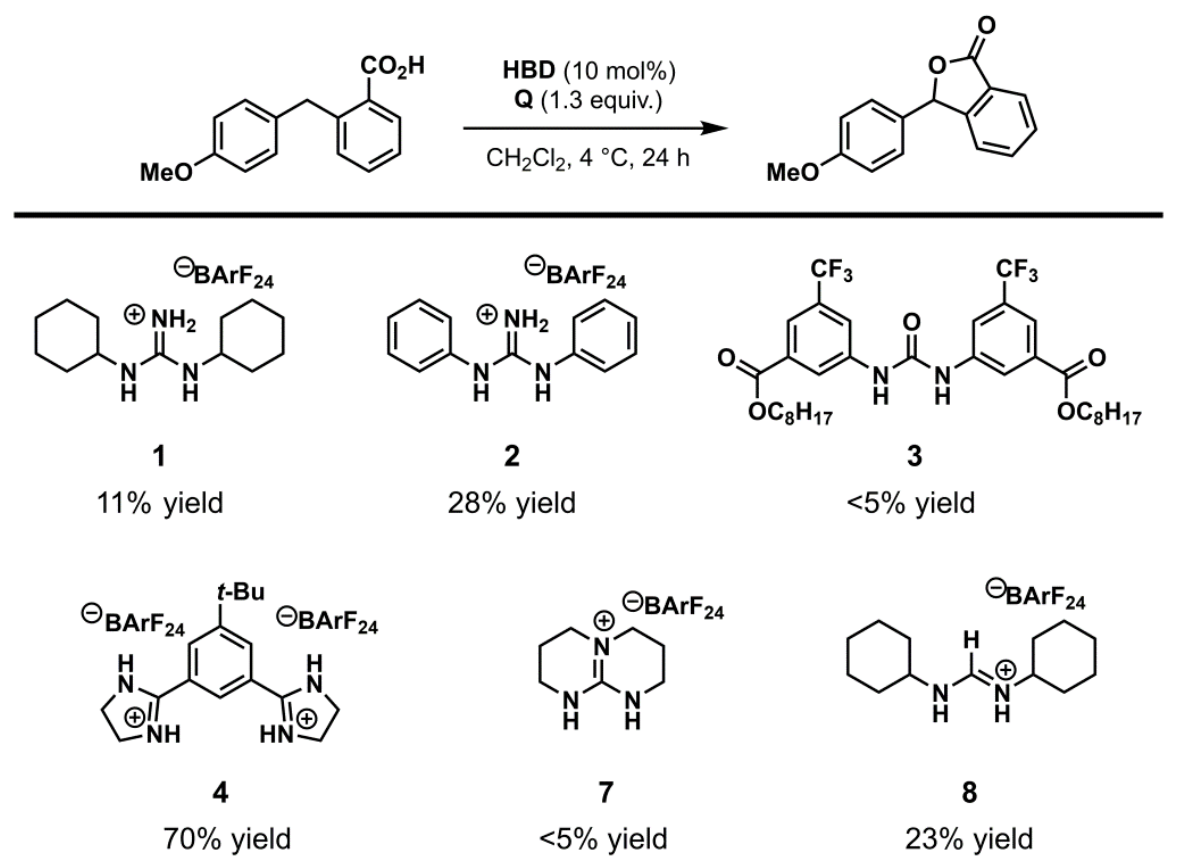

Figure 2.87. Yields of oxidative lactonization after 24 h, catalyzed by HBDs. ${ }^{43}$

Table 2.17. Comparison of thermodynamic and kinetic parameters with yields of oxidative lactonization.

\begin{tabular}{c|cccc}
\hline HBD & $\mathrm{k}_{\mathrm{rel}}\left(\mathrm{s}^{-1}\right)$ & $\mathrm{K}_{3}\left(\mathrm{M}^{-1}\right)$ & $\mathrm{K}_{3} \mathrm{~K}_{4}\left(\mathrm{M}^{-2}\right)$ & yields \\
\hline $\mathbf{3}$ & 1 & $5.6 \times 10^{4}$ & $1.0 \times 10^{7}$ & $0 \%$ \\
$\mathbf{7}$ & $3.6 \times 10^{2}$ & $3.2 \times 10^{5}$ & $2.2 \times 10^{8}$ & $<5 \%$ \\
$\mathbf{1}$ & $4.9 \times 10^{2}$ & $3.4 \times 10^{4}$ & $6.1 \times 10^{8}$ & $11 \%$ \\
$\mathbf{8}$ & $5.2 \times 10^{5}$ & $3.5 \times 10^{4}$ & $2.9 \times 10^{9}$ & $23 \%$ \\
$\mathbf{2}$ & $2.3 \times 10^{7}$ & $3.5 \times 10^{5}$ & $1.8 \times 10^{10}$ & $28 \%$ \\
$\mathbf{4}$ & $9.0 \times 10^{11}$ & $9.2 \times 10^{10}$ & -- & $70 \%$
\end{tabular}

${ }^{43}$ With Dr. David J. Hardee 


\section{2c.2.2 Structure-activity relationship studies on 4}

To further probe the effects of HBD structure on catalytic activity, we examined derivatives of $\mathbf{4}$ in the oxidative lactonization. Bis-amidinium salt 10, which lacks the $t$-butyl substituent present in $\mathbf{4}$, affords the product in near quantitative yield (Figure 2.88) after $24 \mathrm{~h}$, indicating superior rate enhancement relative to 4 . We attribute the diminished activity of 4 to an inductive effect of the $t$-butyl group,${ }^{44}$ which would have a deactivating influence on both the Hbonding strength and electrostatic effect of $\mathbf{4}$. However, a rigorous comparison of $\mathbf{4}$ and $\mathbf{1 0}$ with respect to thermodynamics and kinetics was not possible, because of the very low solubility of 10 in dichloromethane.

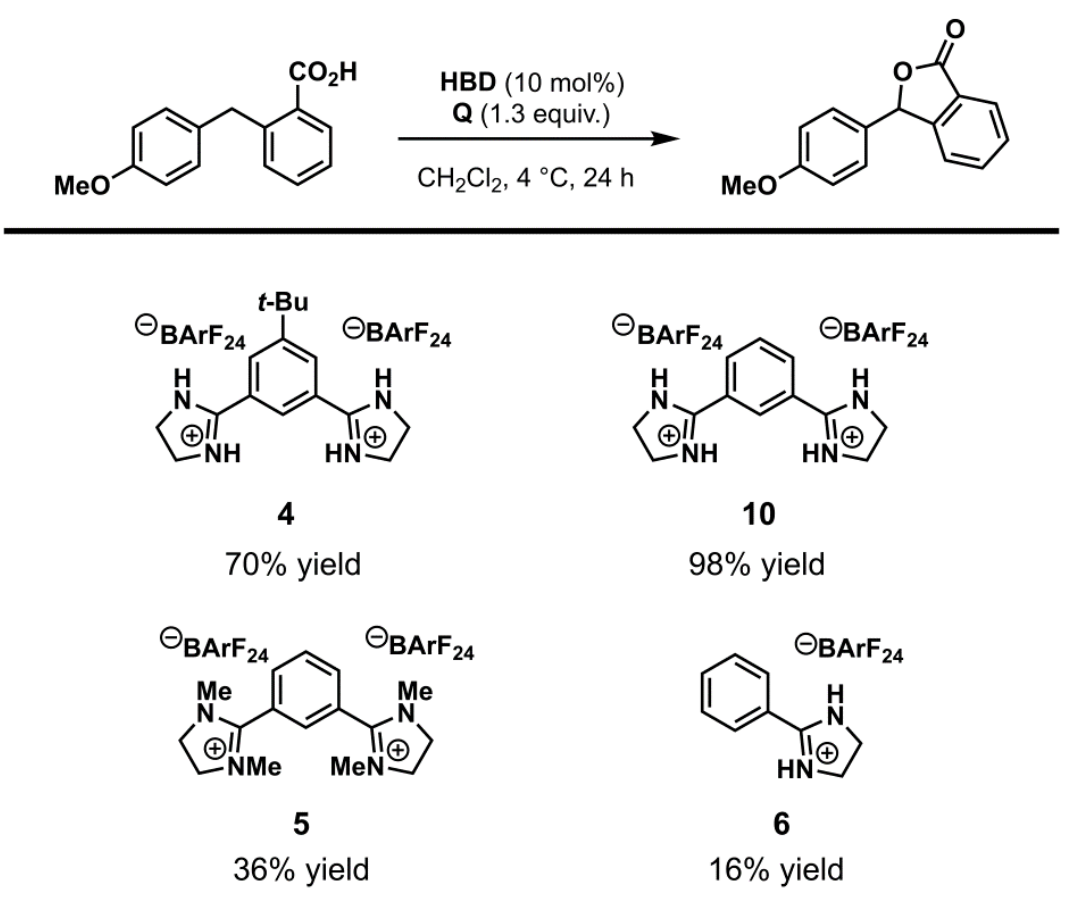

Figure 2.88. Yields obtained with bis-amidinium salts and derivatives thereof.

\footnotetext{
${ }^{44}$ Hansch, C.; Leo, A.; Taft, R. W. Chem. Rev. 1991, 91, 165-195.
} 
The electrochemical experiments reveal that the binding affinity of $\mathbf{4}$ for $\mathbf{Q}^{\cdot-}$ is the result of H-bonding interactions between the two molecules, as tetramethylated bis-amidinium 5which cannot form H-bonds to $\mathbf{Q}^{\mathbf{0}^{-}}$— was found to exhibit weaker binding ability. We wanted to probe whether this effect would hold in the oxidative lactonization as well. When $\mathbf{5}$ is used to catalyze the reaction, significantly reduced yields are obtained than with analogous HBD $\mathbf{1 0}$. This diminished reactivity could be attributed to the aforementioned lack of H-bonding ability. However, the yield with $\mathbf{5}$ is still perhaps higher than expected, especially when compared with the monocationic guanidinium salts that are more effective at binding to $\mathbf{Q}^{\mathbf{*}}$. A different mechanism may be operative when $\mathbf{5}$ is used-as this additive possesses two methylating equivalents, alkylation of the quinone could occur. This would provide a different driving force for the reaction, and may account for the higher yields obtained.

Tethering of the two monocationic subunits also has a substantial effect on the binding affinity for $\mathbf{Q}^{\bullet-}$, as determined electrochemically. To examine the effect of this linkage on the lactonization, we examined amidinium salt $\mathbf{6}$ - the monomeric subunit of $\mathbf{1 0}$-which affords the lactone in decreased yield after $24 \mathrm{~h}$. This decrease in reactivity is consistent with the diminished binding ability of 6 relative to 4 . This result also fits in neatly with respect to the overall trend in yield across the full series of HBDs, with $\mathbf{6}$ giving both intermediate yield and binding strength between $\mathbf{1}$ and $\mathbf{8}$ (Table 2.18). 
Table 2.18. Comparison of thermodynamic and kinetic parameters with yields of oxidative lactonization for structure-activity relationship based on $\mathbf{4}$

\begin{tabular}{cccc}
\hline HBD & $\mathrm{K}_{3}\left(\mathrm{M}^{-1}\right)^{\mathrm{b}}$ & $\mathrm{K}_{3} \mathrm{~K}_{4}\left(\mathrm{M}^{-2}\right)$ & yields \\
\hline $\mathbf{1}$ & $3.4 \times 10^{4}$ & $6.1 \times 10^{8}$ & $11 \%$ \\
$\mathbf{6}$ & $1.2 \times 10^{4}$ & $1.5 \times 10^{9}$ & $16 \%$ \\
$\mathbf{8}$ & $3.5 \times 10^{4}$ & $2.9 \times 10^{9}$ & $23 \%$ \\
$\mathbf{5}$ & $5.7 \times 10^{4}$ & -- & $36 \%$ \\
$\mathbf{4}$ & $9.2 \times 10^{10}$ & -- & $70 \%$ \\
$\mathbf{1 0}$ & n.d & -- & $98 \%$
\end{tabular}

\section{2c.2.3 Effect of dimeric HBDs}

Based on our observation that two guanidinium salts bind to $\mathbf{Q}^{\bullet-}$ in a $2: 1$ complex, we hypothesized that tethering two of these HBDs would afford a more effective catalyst. Guanidinium salt $\mathbf{1 1}$ - a dimer of $\mathbf{1}$-indeed provides the lactone in improved yield at $24 \mathrm{~h}$ reactions times relative to the monomeric guanidinium salt, albeit in diminished yield relative to bis-amidinium salt 10 (Figure 2.89). The relationship between $\mathbf{1}$ and $\mathbf{1 1}$ further supports our conclusion that dicatonic scaffolds are necessary to achieve improved lactonization rates by virtue of more effective stabilization of $\mathbf{Q}^{\bullet-}$.

To segregate the effect of tethering from the effect of increased charge, we prepared linked urea-guanidinium 12 which tethers two HBDs in a monocationic framework. Relative to monomeric 1, no improvement in yield is observed. This demonstrates that the effect of tethering alone is minimal, and substantially affects the reaction rate only when such a linkage affords a dicationic HBD. 


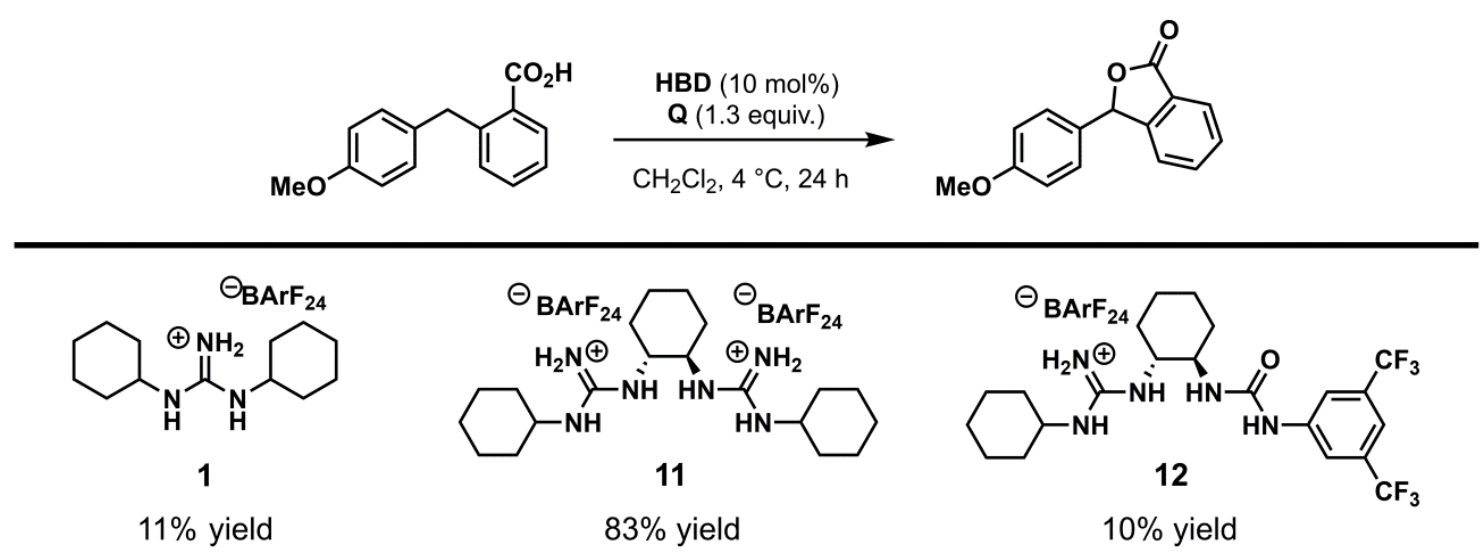

Figure 2.89. Oxidative lactonization catalyzed by linked HBDs.

\section{2c.3 Kinetic isotope effect experiment and proposed mechanism}

A kinetic isotope effect (KIE) experiment for the oxidative lactonization allowed us to probe the mechanism for the oxidative lactonization and determine whether it indeed involves electron transfer (Figure 2.90). ${ }^{45}$ An intramolecular experiment with $\mathbf{1 3}$ and its deuterated analog reveals a large, primary $\operatorname{KIE}\left(\mathrm{k}_{\mathrm{H}} / \mathrm{k}_{\mathrm{D}}=7.7\right)$ when the reaction is catalyzed by $\mathbf{1 0}$, consistent with cleavage of the methylene $\mathrm{C}-\mathrm{H} / \mathrm{D}$ bond in the rate-limiting step. Other quinone-mediated oxidations on related benzylic substrates have also exhibited large primary KIEs consistent with tunneling. ${ }^{46}$ Although this KIE indicates that electron transfer does not constitute the ratelimiting step, it does not exclude involvement of electron transfer in the mechanism. ${ }^{47} \mathrm{An}$ alternative mechanistic scenario with no electron transfer involves direct hydride transfer from the substrate. However, the clear correlation between the correlation between the rate of lactonization and the kinetics of a homogenous electron transfer is consistent with a mechanism

\footnotetext{
${ }^{45}$ Performed by Dr. David J. Hardee.

${ }^{46}$ Jung, H. H.; Floreancig, P. E. Tetrahedron 2009, 65, 10830-10836.

${ }^{47}$ Guo, X.; Zipse, H.; Mayr, H. J. Am. Chem. Soc. 2014, 136, 13863-13873.
} 
involving electron transfer (Figure 2.91). H-bonding interactions could modulate a preequilibrium electron transfer step, which is followed by rate-limiting $\mathrm{H}$-atom transfer. Based on the electrochemical experiments, pre-association between the HBD and neutral quinone is also proposed.
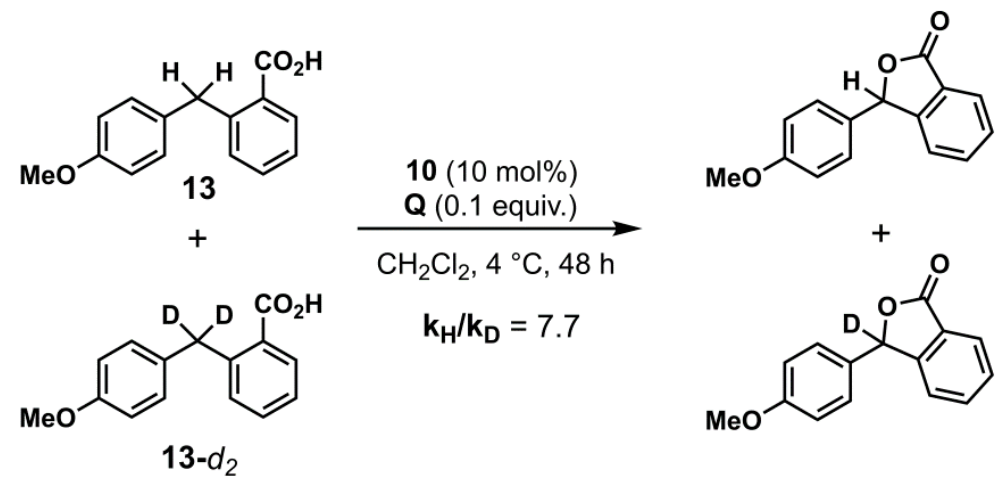

$\mathbf{k}_{\mathrm{H}} / \mathbf{k}_{\mathrm{D}}=7.7$

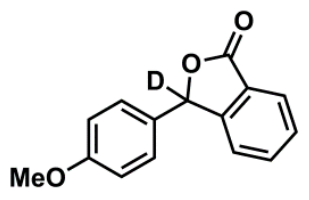

Figure 2.90. Intramolecular KIE experiment.

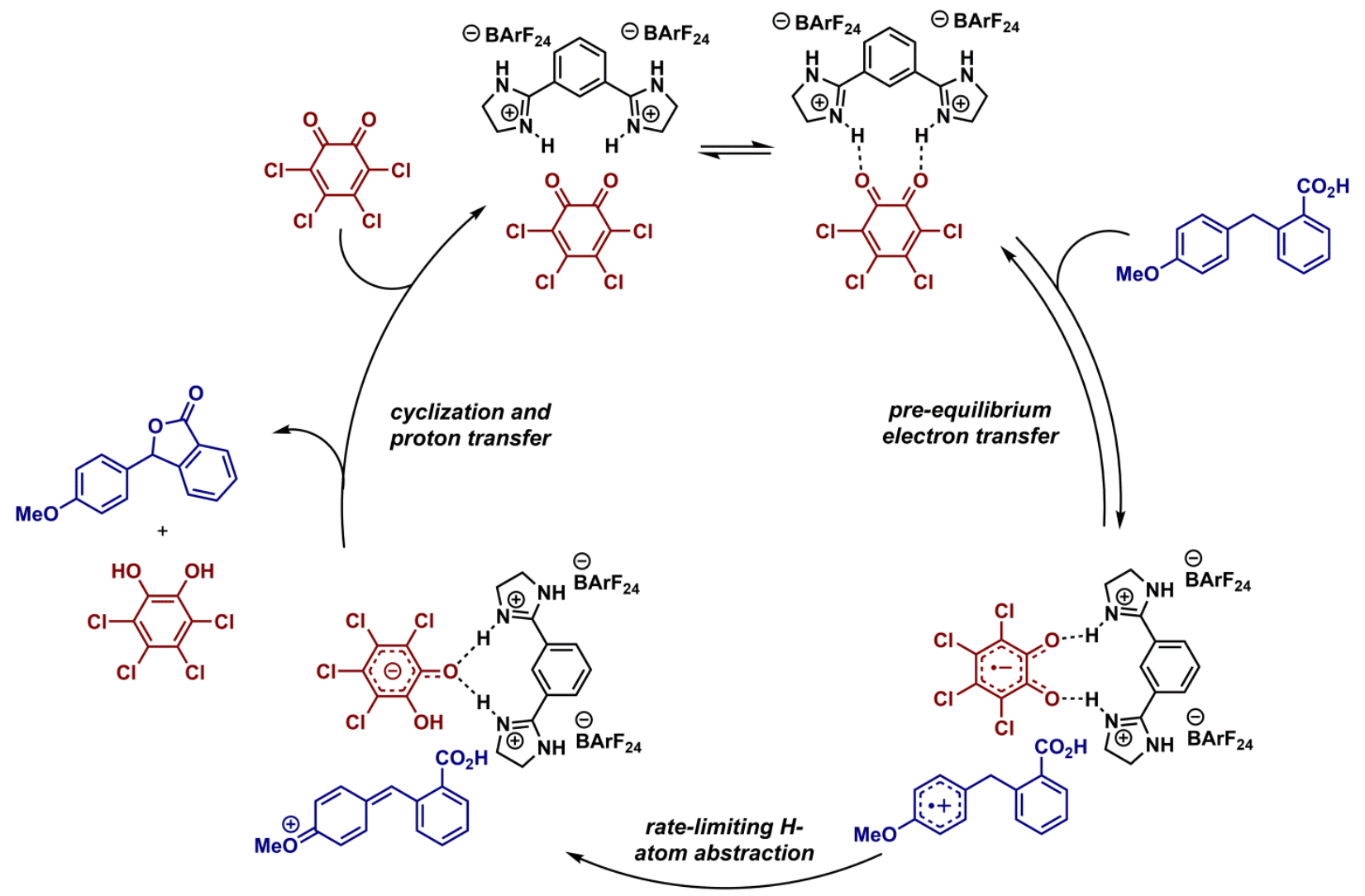

Figure 2.91. Proposed catalytic cycle for oxidative lactonization mediated by $o$-chloranil. 


\section{2c.4 Experimental details}

\section{2c.4.1 Procedures for the syntheses of HBDs 10-12}

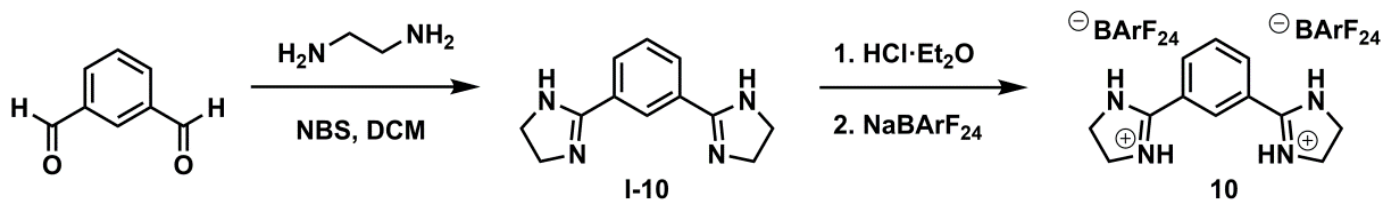

\section{1,3-bis(4,5-dihydro-1H-imidazol-2-yl)benzene (I-10)}

1,3-bis(4,5-dihydro-1H-imidazol-2-yl)benzene (I-10) is a known compound and was synthesized from commercially available isophthalaldehyde as reported in the literature. ${ }^{48}$ Spectral data matched that which was previously reported.

\section{2,2'-(1,3-phenylene)bis(4,5-dihydro-1H-imidazol-3-ium) tetrakis(3,5-}

\section{bis(trifluoromethyl)phenyl)borate (10)}

$\mathbf{1 0}$ is a known compound and was synthesized as reported in the literature. ${ }^{48}$ Spectral data matched that which was previously reported.

\footnotetext{
${ }^{48}$ Huynh, P. N. H.; Walvoord, R. R.; Kozlowski, M. C. J. Am. Chem. Soc. 2012, 134, 15621-15623.
} 

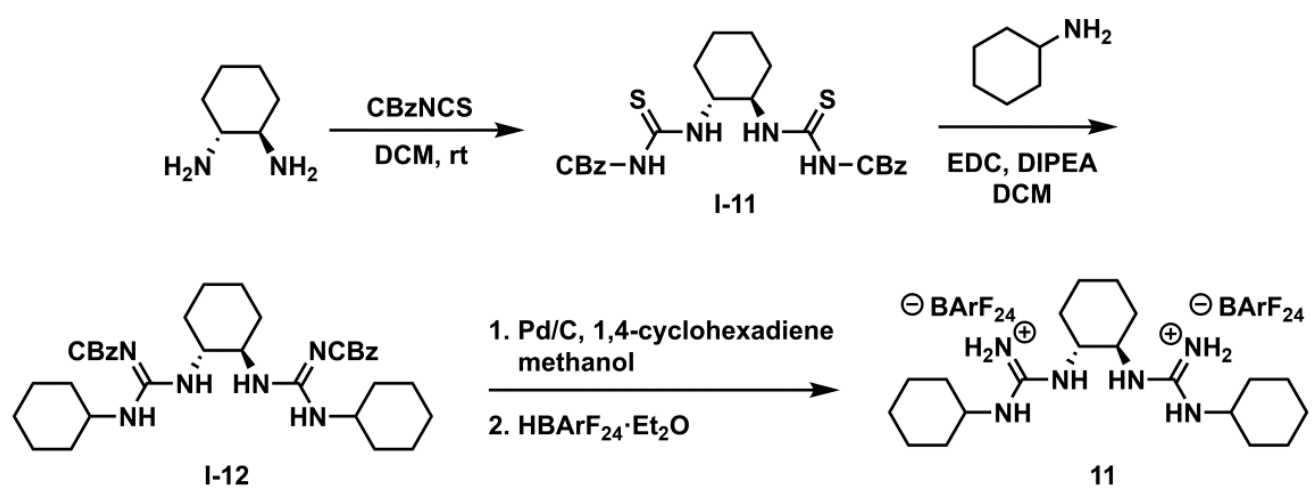

To a cooled solution $\left(0^{\circ} \mathrm{C}\right)$ of $(\mathrm{R}, \mathrm{R})$-diaminocyclohexane $(0.5 \mathrm{~g}, 4.4 \mathrm{mmol}, 1.0$ equiv) in DCM (20 mL) was added a solution of benzyloxyisothiocyanate $(1.9 \mathrm{~g}, 9.7 \mathrm{mmol}, 2.2$ equiv) in DCM $(2 \mathrm{~mL})$. The reaction mixture was stirred for $14 \mathrm{~h}$ and was concentrated via rotary evaporation in vacuo. Column chromatography on silica gel (10-40\% EtOAc in hexanes with $1 \%$ $\mathrm{MeOH})$ yielded the product I-11 as a white solid (1.49 g, 68\% yield).

${ }^{1}$ H NMR (500 MHz, Acetone- $\left.d_{6}\right) \delta 9.88(\mathrm{~s}, 2 \mathrm{H}), 9.74$ (s, $\left.2 \mathrm{H}\right), 7.44-7.33$ (m, $\left.10 \mathrm{H}\right), 5.19$ (s, $\left.4 \mathrm{H}\right)$. 4.63-4.60 (m, 2 H), 2.24-2.20 (m, 2 H), 1.80-1.79 (m, 2 H), 1.51-1.44 (m, 4 H);

${ }^{13} \mathbf{C}$ NMR $\left\{{ }^{1} \mathbf{H}\right\}\left(125 \mathrm{MHz}\right.$, Acetone- $\left.d_{6}\right) \delta 179.6,153.0,135.6,128.5,128.3,128.1,67.3,57.7,30.9$, 24.2;

IR (film) $v_{\max } 1724,1546,1536,1454,1399,1393,1361,1345,1257,1225,1188,1026,981$, 769, 735, 699;

MS (ESI-TOF) calcd. for $\mathrm{C}_{24} \mathrm{H}_{28} \mathrm{~N}_{4} \mathrm{O}_{4} \mathrm{~S}_{2}\left[\mathrm{M}+\mathrm{H}^{+}\right]$501.1630, found 501.1565 .

I-12 was prepared by an adaptation of an existing procedure. ${ }^{49}$ To a solution of I-11 (100 $\mathrm{mg}, 0.2 \mathrm{mmol}, 1.0$ equiv.) in DCM was added cyclohexylamine ( $69 \mu \mathrm{L}, 0.6 \mathrm{mmol}, 3.0$ equiv.) and DIPEA (105 $\mu \mathrm{L}, 0.6 \mathrm{mmol}, 3.0$ equiv.). The reaction mixture was cooled to $0{ }^{\circ} \mathrm{C}$ and $\mathrm{EDC}$ (153 mg, $0.8 \mathrm{mmol}, 4.0$ equiv.) was added. The reaction mixture was warmed to $\mathrm{rt}$ over 30

${ }^{49}$ Linton, B. R.; Carr, A. J.; Orner, B. P.; Hamilton, A. D. J. Org. Chem. 2000, 65, 1566-1568. 
minutes and was stirred overnight. The reaction mixture was transferred to a separatory funnel and diluted with DCM. The organic phase was extracted with DCM and was washed with $1 \%$ aqueous $\mathrm{HCl}$, sat. aq. sodium bicarbonate, and brine. The combined organic phases were dried over $\mathrm{Na}_{2} \mathrm{SO}_{4}$ and were concentrated in vacuo. Column chromatography on silica gel $(0-30 \%$ ethyl acetate in hexanes) afforded the product I-12 (67.8 $\mathrm{mg}, 54 \%$ yield).

A round-bottomed flask was charged with Pd/C (140 mg, $200 \mathrm{wt} \%$ with respect to I-12), which was slurried with EtOAc. MeOH $(2.2 \mathrm{~mL})$ was slowly added to the slurry, and I-12 (68 $\mathrm{mg}, 0.11 \mathrm{mmol}, 1.0$ equiv.) was transferred to the flask using $\mathrm{MeOH}(1 \mathrm{~mL})$. The flask was submerged in a rt water bath and 1,4-cyclohexadiene $(0.42 \mathrm{~mL}, 2.2 \mathrm{mmol}, 40$ equiv. $)$ was added. The reaction was stirred and monitored by MS until no starting material remained ( 12-24 h). The reaction mixture was filtered through Celite and rinsed with $\mathrm{MeOH}$, and was concentrated via rotary evaporation in vacuo. The residue was suspended in $\mathrm{Et}_{2} \mathrm{O}$ and $\mathrm{HBArF}_{24} \cdot \mathrm{Et}_{2} \mathrm{O}(206 \mathrm{mg}$, $0.22 \mathrm{mmol}, 2.0$ equiv.) was added. The suspension was stirred for $15 \mathrm{~min}$ and the reaction mixture was then filtered through Celite. The filtrate was concentrated via rotary evaporation in vacuo to afford $\mathbf{1 1}$ as a colorless oil that formed a foamy solid under vacuum (153 $\mathrm{mg}, 67 \%$ yield).

${ }^{1}$ H NMR (500 MHz, Acetone- $\left.d_{6}\right) 7.97$ (s, 4 H), 7.80 (s, 16 H), 7.70 (s, 8 H), 3.88-3.87 (m, 2 H), 3.65-3.64 (m, 2 H), 2.23-2.20 (m, 2H), 2.09-2.05 (m, 8 H), 1.75 (m, 4 H), 1.58-1.56 (m, 4 H), 1.42-1.31 (m, 8 H), 1.18-1.15 (m, 2 H);

${ }^{13} \mathbf{C}$ NMR $\left\{{ }^{1} \mathbf{H}\right\}\left(125 \mathrm{MHz}\right.$, Acetone- $\left.d_{6}\right) \delta 161.7$ (q, J = 49.6 Hz), 154.8, 142.0, 134.6, 132.9, 131.6 (q, J = 33.3), $129.1(q, J=32.6), 124.5$ (q, $J=271.8 \mathrm{~Hz}), 55.6,51.1,32.5,29.3,24.7,24.3,23.9$; IR (film) $v_{\max } 2944,2866,1686,1609,1454,1354,1274,1161,1117,1028,887,839,745,712$, 670,482 
MS (ESI-TOF) calcd. for $\mathrm{C}_{20} \mathrm{H}_{39} \mathrm{~N}_{6}\left[\mathrm{M}-\mathrm{H}^{+}-2 \mathrm{BAr}^{\mathrm{F}}{ }_{24}\right] 363.3236$, found 363.3295.
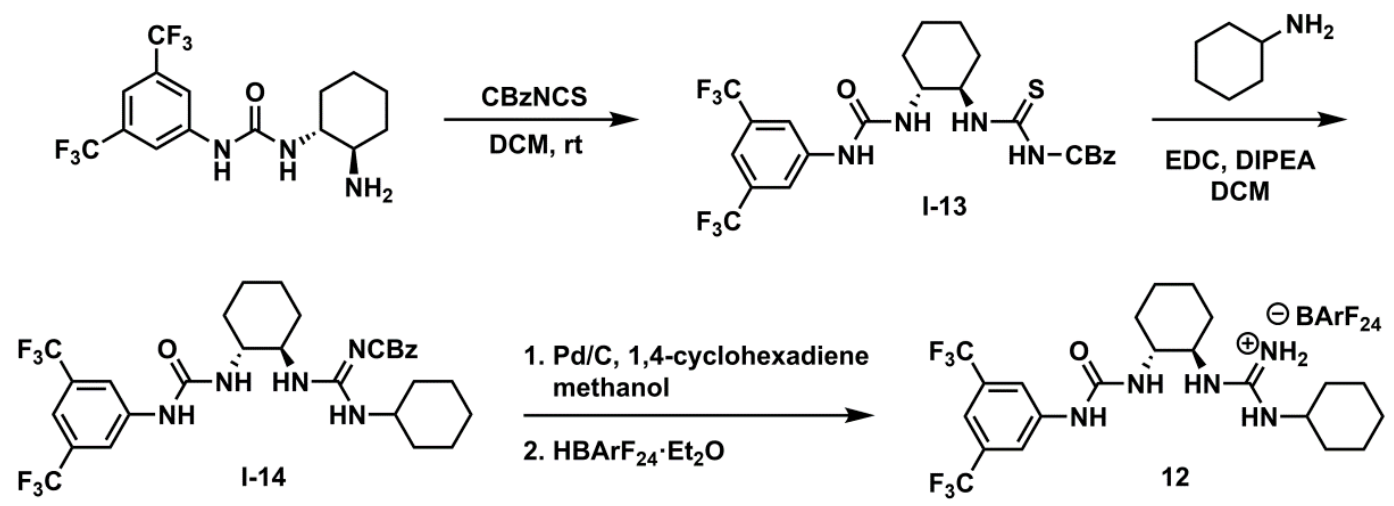

A round-bottomed flask was charged with the primary aminourea ${ }^{50}(116 \mathrm{mg}, 0.29 \mathrm{mmol}$, 1.0equiv.), and DCM (2 mL) was added. Triethylamine (45 $\mu \mathrm{L}, 0.32 \mathrm{mmol}, 1.1$ equiv.) was added, followed by benzyloxyisothiocyanate $(62 \mathrm{mg}, 0.32 \mathrm{mmol}, 1.1$ equiv.) as a solution in DCM $(0.9 \mathrm{~mL})$. After stirring for $12 \mathrm{~h}$, the reaction mixture was transferred to a separatory funnel. The organic phase was washed with $1 \mathrm{M} \mathrm{HCl}$ (aq.) $(3 \times 10 \mathrm{~mL})$ and brine. The organic phase was dried over $\mathrm{Na}_{2} \mathrm{SO}_{4}$ and was concentrated via rotary evaporation in vacuo. Column chromatography on silica gel (0-20\% ethyl acetate in hexanes) afforded the product as a white solid (111 mg, 68\% yield).

${ }^{1}$ H NMR (500 MHz, Acetone-d $\left.d_{6}\right) \delta 9.92$ (br. s, $\left.1 \mathrm{H}\right), 9.74(\mathrm{~s}, 1 \mathrm{H}), 8.61$ (s, $\left.1 \mathrm{H}\right), 8.17$ (s, $\left.2 \mathrm{H}\right)$, $7.52(\mathrm{~s}, 1 \mathrm{H}), 7.38-7.31(\mathrm{~m}, 5 \mathrm{H}), 6.13(\mathrm{~d}, 1 \mathrm{H}, \mathrm{J}=8.4), 5.20(\mathrm{~d}, 1 \mathrm{H}, \mathrm{J}=12.5), 5.12(\mathrm{~d}, 1 \mathrm{H}, \mathrm{J}=$ 12.0), 4.34-4.27 (m, $1 \mathrm{H}), 3.93-3.87$ (m, $1 \mathrm{H}), 2.92-2.27$ (m, $1 \mathrm{H}), 2.12-2.09$ (m, $1 \mathrm{H}), 1.78-$ $1.76(\mathrm{~m}, 2 \mathrm{H}), 1.45-1.34(\mathrm{~m}, 4 \mathrm{H})$;

${ }^{13} \mathbf{C}$ NMR $\left\{{ }^{1} \mathbf{H}\right\}\left(125 \mathrm{MHz}\right.$, Acetone- $\left.d_{6}\right) \delta 179.8,154.4,153.1,142.7,135.5,131.5,131.3,128.4$ $128.3,128.1,117.7,113.9,67.3,58.7,52.9,32.8,31.0,24.5,24.4$

\footnotetext{
${ }^{50}$ Brown, A. R.; Kuo, W.-H.; Jacobsen, E. N. J. Am. Chem. Soc. 2010, 132, 9286-9288.
} 
IR (film) $v_{\max } 2937,2860,1721,1657,1531,1475,1449,1386,1277,1228,1176,1130,1026$, $882,732,701,682$

MS (ESI-TOF) calcd. for $\mathrm{C}_{24} \mathrm{H}_{24} \mathrm{~F}_{6} \mathrm{~N}_{4} \mathrm{O}_{3} \mathrm{~S}\left[\mathrm{M}+\mathrm{H}^{+}\right]$563.1552, found 563.1585 .

To a solution of $\mathbf{I}-\mathbf{1 2}(110 \mathrm{mg}, 0.2 \mathrm{mmol}, 1.0$ equiv) in dichloromethane $(0.1 \mathrm{M})$ was added cyclohexylamine ( $69 \mu \mathrm{L}, 0.6 \mathrm{mmol}, 3.0$ equiv) and DIPEA ( $0.1 \mathrm{~mL}, 0.6 \mathrm{mmol}$, 3.0equiv). The stirred solution was cooled to $0{ }^{\circ} \mathrm{C}$ and $\mathrm{EDC}(0.12 \mathrm{~g}, 0.6 \mathrm{mmol}, 3.0$ equiv. $)$ was added. The reaction mixture was allowed to warm to room temperature, and was stirred until the reaction was complete by mass spectrometry. The reaction mixture was transferred to a separatory funnel and was diluted with DCM. The organic phase was washed with $1 \%$ aqueous $\mathrm{HCl}(2 \times 20 \mathrm{~mL})$, and brine $(1 \times 20 \mathrm{~mL})$. The organic phase was dried over $\mathrm{Na}_{2} \mathrm{SO}_{4}$ and was concentrated via rotary evaporation in vacuo. Column chromatography on silica gel (0-20\% EtOAc in hexanes) yielded the product as a colorless oil. (MS (ESI-TOF) calcd. for $\mathrm{C}_{24} \mathrm{H}_{24} \mathrm{~F}_{6} \mathrm{~N}_{4} \mathrm{O}_{3} \mathrm{~S}\left[\mathrm{M}+\mathrm{H}^{+}\right] 563.1552$, found 563.1585.)

A round-bottomed flask was charged with Pd/C (160 mg, $200 \mathrm{wt} \%$ with respect to I-13), which was slurried with EtOAc. MeOH $(1.6 \mathrm{~mL})$ was slowly added to the slurry, and I-13 (79 $\mathrm{mg}, 0.13 \mathrm{mmol}, 1.0$ equiv.) was transferred to the flask using $\mathrm{MeOH}(1 \mathrm{~mL})$. The flask was submerged in a rt water bath and 1,4-cyclohexadiene $(0.24 \mathrm{~mL}, 2.5 \mathrm{mmol}, 20$ equiv.) was added. The reaction was stirred and monitored by MS until no starting material remained. The reaction mixture was filtered through Celite and rinsed with $\mathrm{MeOH}(\sim 25 \mathrm{~mL})$, and the filtrate was concentrated via rotary evaporation in vacuo. The residue was taken up in $\mathrm{Et}_{2} \mathrm{O}$ and $\mathrm{HBArF}_{24} \cdot \mathrm{Et}_{2} \mathrm{O}(122 \mathrm{mg}, 0.13 \mathrm{mmol}, 1.0$ equiv.) was added. The suspension was stirred for 10 min and was concentrated via rotary evaporation in vacuo. The residue was dissolved in DCM 
and filtered through Celite, and was concentrated via rotary evaporation in vacuo to afford the product a brown oil (137 $\mathrm{mg}, 78 \%$ yield).

${ }^{1}$ H NMR $\left(500 \mathrm{MHz}\right.$, Acetone- $\left.d_{6}\right) \delta 8.68(\mathrm{~s}, 1 \mathrm{H}), 8.14(\mathrm{~s}, 2 \mathrm{H}), 7.92(\mathrm{~s}, 1 \mathrm{H}), 7.80(\mathrm{~s}, 8 \mathrm{H}), 7.68$ (s, $4 \mathrm{H}), 7.60$ (s, $1 \mathrm{H}), 7.08$ (s, $2 \mathrm{H}), 6.47$ (s, $1 \mathrm{H}), 3.79$ (br. s., $1 \mathrm{H}), 3.67-3.63$ (m, $1 \mathrm{H}), 3.59-3.56$ (m, $1 \mathrm{H}), 2.17-1.11(\mathrm{~m}, 18 \mathrm{H})$;

${ }^{13} \mathbf{C} \mathbf{N M R}\left\{{ }^{1} \mathbf{H}\right\}\left(125 \mathrm{MHz}\right.$, Acetone- $\left.d_{6}\right) \delta 161.7(\mathrm{q}, \mathrm{J}=49.6 \mathrm{~Hz}), 156.0,154.8,142.0,134.6,132.9$, $131.6(q, J=33.3), 129.1(q, J=32.6), 124.6,124.5$ (q, $\mathrm{J}=271.8 \mathrm{~Hz}), 122.4,119.9,118.1,117.5$, $114.6,52.4,50.8,32.4,24.8,24.3,23.8,23.2$

IR (film) $v_{\max } 2943,2866,1636,1569,1475,1453,1386,1354,1315,1273,1116,1041,943$, $885,839,745,712,669,579,448$

MS (ESI-TOF) calcd. for $\mathrm{C}_{22} \mathrm{H}_{30} \mathrm{~F}_{6} \mathrm{~N}_{5} \mathrm{O}\left[\mathrm{M}-2 \mathrm{BAr}_{2}^{\mathrm{F}}\right]$ 494.2354, found 494.2518.

\section{2c.4.2 Oxidative lactonization procedures and substrate synthesis}

\section{Synthesis of oxidative lactonization substrate}

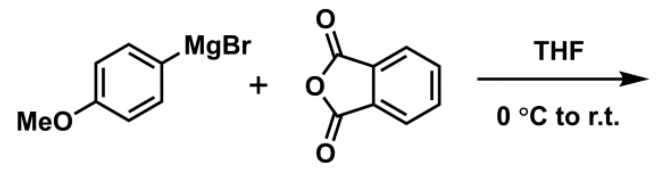

\section{2-(4-Methoxybenzoyl)benzoic acid (I-15)}

4-Methoxyphenylmagnesium bromide ( $8 \mathrm{~mL}, 0.5 \mathrm{M}$ in THF, $4.0 \mathrm{mmol}, 1.0$ equiv) was added dropwise to phthalic anhydride $\left(711 \mathrm{mg}, 4.8 \mathrm{mmol}, 1.2\right.$ equiv) in THF $(10 \mathrm{~mL})$ at $0{ }^{\circ} \mathrm{C}$. The reaction was warmed to $\mathrm{rt}$ and stirred for $12 \mathrm{~h}$. The mixture was quenched with $1 \mathrm{M} \mathrm{HCl}(\mathrm{aq})$ and extracted with $\mathrm{Et}_{2} \mathrm{O}$. The organic phase was extracted twice with $1 \mathrm{M} \mathrm{NaOH}(\mathrm{aq})$ and the aqueous extracts were acidified with concentrated $\mathrm{HCl}$. The resulting mixture was extracted with $\mathrm{Et}_{2} \mathrm{O}$, washed with brine, dried over $\mathrm{MgSO}_{4}$, and concentrated in vacuo to yield the title 
compound as a white solid (778 $\mathrm{mg}, 3.03 \mathrm{mmol}, 76 \%$ yield). Spectral data matched that which has been previously reported. ${ }^{51}$

\section{2-(4-Methoxybenzyl)benzoic acid (13)}

Trifluoroacetic acid $(3 \mathrm{~mL})$ was added dropwise to 2-(4-methoxybenzoyl)benzoic acid (778 mg, 3.04mmol, 1.0 equiv) and triethylsilane $(1.45 \mathrm{~mL}, 9.1 \mathrm{mmol}, 3.0$ equiv) in chloroform $(3 \mathrm{~mL})$ at $0{ }^{\circ} \mathrm{C}$. The solution was then heated at reflux for $18 \mathrm{~h}$. After cooling to rt, the reaction was diluted with $\mathrm{Et}_{2} \mathrm{O}$ and washed twice with $1 \mathrm{M} \mathrm{HCl}$ (aq). The organic phase was extracted twice with $1 \mathrm{M} \mathrm{NaOH}$ (aq) and the aqueous extracts were acidified with concentrated $\mathrm{HCl}$. The resulting mixture was extracted with $\mathrm{Et}_{2} \mathrm{O}$, washed with brine, dried with $\mathrm{MgSO}_{4}$, and concentrated in vacuo to yield the title compound as a white solid (619 mg, $2.56 \mathrm{mmol}, 84 \%$ yield). Spectral data matched that which has been previously reported. ${ }^{52}$

\section{Catalyst rate comparison}

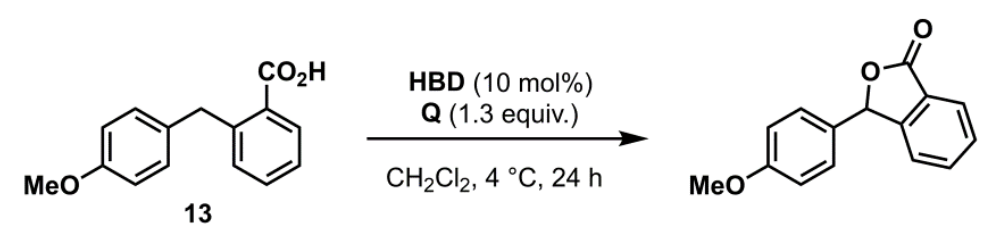

A solution of $o$-chloranil $(16.0 \mathrm{mg}, 0.065 \mathrm{mmol}, 1.3$ equiv) in $\mathrm{DCM}(0.1 \mathrm{~mL})$ was added to a solution of (12.2 mg, $0.05 \mathrm{mmol}, 1.0$ equiv) and $\mathrm{HBD}(10 \mathrm{~mol} \%)$ in $\mathrm{CH}_{2} \mathrm{Cl}_{2}(0.5 \mathrm{~mL})$ at +4 ${ }^{\circ} \mathrm{C}$. After $24 \mathrm{~h}$ the reaction was quenched with a sat. aq. ascorbic acid solution and extracted with EtOAc. The organic phase was washed with brine, dried with $\mathrm{MgSO}_{4}$, and concentrated. Yields

\footnotetext{
${ }^{51}$ Miao, J.; Ge, H. Org. Lett. 2013, 15, 2930-2933.

${ }^{52}$ Doering, W.v.E.; Kitagawa, T. J. Am. Chem. Soc., 1991, 113, 4288-4297.
} 
were determined by ${ }^{1} \mathrm{H}$ NMR analysis of the crude reaction versus an internal NMR standard $\left(\mathrm{Bn}_{2} \mathrm{O}\right)$. The lactone product is a known compound and spectral data matched that which has been previously reported. ${ }^{53}$

\section{2c.4.3 KIE experiment}

Synthesis of deuterated substrate

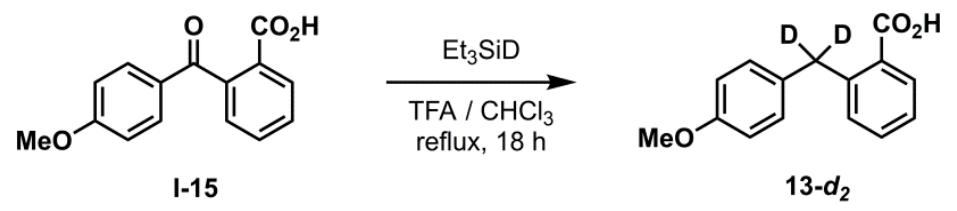

\section{2-((4-Methoxyphenyl)methyl- $\left.d_{2}\right)$ benzoic acid (13- $\left.d_{2}\right)$}

Trifluoroacetic acid $(0.15 \mathrm{~mL})$ was added dropwise to 2-(4-methoxybenzoyl)benzoic acid (38 mg, $0.15 \mathrm{mmol}, 1.0$ equiv) and triethyl(silane-d) (72 $\mu \mathrm{L}, 0.45 \mathrm{mmol}, 3.0$ equiv) in $\mathrm{CHCl}_{3}$ $(0.15 \mathrm{~mL})$ at $0{ }^{\circ} \mathrm{C}$. The solution was then heated at reflux for $18 \mathrm{~h}$. After cooling to rt, the reaction was diluted with $\mathrm{Et}_{2} \mathrm{O}$ and washed twice with $1 \mathrm{M} \mathrm{HCl}$ (aq). The organic phase was extracted twice with $1 \mathrm{M} \mathrm{NaOH}(\mathrm{aq})$ and the aqueous extracts were acidified with concentrated $\mathrm{HCl}$. The resulting mixture was extracted with $\mathrm{Et}_{2} \mathrm{O}$, washed with brine, dried over $\mathrm{MgSO}_{4}$, and concentrated in vacuo to yield the title compound as a white solid $(30 \mathrm{mg}, 0.12 \mathrm{mmol}, 82 \%$ yield).

\section{Kinetic isotope experiment}

\section{Catalyzed with 10}

A solution of $o$-chloranil $(2.5 \mathrm{mg}, 0.01 \mathrm{mmol}, 0.1$ equiv) in DCM $(0.2 \mathrm{~mL})$ was added to a solution of 2-((4-methoxyphenyl)methyl- $\left.d_{2}\right)$ benzoic acid (12.2 $\mathrm{mg}, 0.05 \mathrm{mmol}, 0.5$ equiv), 2-

\footnotetext{
${ }^{53}$ Gerbino, D. C.; Augner, D.; Slavov, N.; Schmalz, H. G. Org. Lett. 2012, 14, 2338-2341.
} 
((4-methoxyphenyl)methyl- $\left.\mathrm{H}_{2}\right)$ benzoic acid ( $12.2 \mathrm{mg}, 0.05 \mathrm{mmol}, 0.5$ equiv), and bis-amidinium salt 10 (19.4 mg, $0.01 \mathrm{mmol}, 10 \mathrm{~mol} \%)$ in DCM $(0.8 \mathrm{~mL})$ at $+4{ }^{\circ} \mathrm{C}$. After $48 \mathrm{~h}$, the reaction was quenched with a sat. aq. ascorbic acid solution and extracted with EtOAc. The organic phase was washed twice with $1 \mathrm{M} \mathrm{NaOH}(\mathrm{aq})$, washed with brine, dried over $\mathrm{MgSO}_{4}$, and concentrated in vacuo to yield the lactone product. The average H/D product ratio for two separate runs was determined by integration of the ${ }^{1} \mathrm{H}$ NMR spectra in $\mathrm{CDCl}_{3}$ with $\mathrm{d}^{1}=10 \mathrm{~s}$. $\mathrm{Pdt}_{\mathrm{H}} / \mathrm{Pdt}_{\mathrm{D}}=7.7$.

\section{Outlook for future work}

\section{2d.1 Expanding the scope of HBD-coupled electron transfer}

\section{2d.1.1 Outlook for investigation with respect to HBD design}

The electrochemical and kinetic studies described herein will continue to inform further development of catalysts for HBD-coupled electron transfer. Specifically, the results obtained from this mechanistic study highlight the promise of dicatonic HBDs as catalysts coupled to electron transfer, which encourages additional investigation of these and related structures. Broadening the substrate scope for HBD-coupled electron transfer will likely require further enhancement of binding between $\mathbf{Q}^{\bullet-}$ and the HBD—namely, by increasing $\Delta \mathrm{G}_{\text {bind }}$, H-bonding will be able to surmount the thermodynamic penalties presented by more difficult electron transfer reactions (Figure 2.3). In order to achieve this enhancement, significant perturbations to the H-bonding motif will need to be made. Preliminary results in this area, as well as results obtained with new dicationic scaffolds, are presented in section 2d.2.2.

Eventually, however, an improvement in H-bonding ability by virtue of increased acidity may cause a mechanistic shift to PCET, as opposed to HBD-coupled electron transfer. To avoid undesirable protonation of $\mathbf{Q}^{\circ-}$, enhanced binding could be achieved through other secondary 
interactions (e.g. pi-pi interactions) which could be incorporated into the H-bonding scaffold. For example, the redox chemistry of flavins is known to be affected by pi-stacking, as interaction of the flavin with an electron-rich aromatic system decreases the reduction potential of the flavin oxidant. ${ }^{54}$ We have conducted preliminary experiments indicating that a converse effect may be achieved with quinones - that interaction of the quinone with an electron-deficient arene has a beneficial, stabilizing effect on $\mathbf{Q}^{\bullet-}$ (Figure 2.92). A study of the electron transfer reaction between $p$-fluoranil and $N, N^{\prime}$-tetramethylphenylenediamine (TMPD) qualitatively shows that guanidinium salts can shift the electron transfer equilibrium to favor the ionized state, with the greatest effect observed when guanidinium salts bearing electron-deficient arenes are used. A systematic investigation of such guanidinium ions (or other, appropriately substituted dicationic HBDs) would allow for quantification of the effect of pi-stacking interactions on the kinetics and thermodynamics of HBD-coupled electron transfer, potentially leading to the development of more potent HBDs.

\footnotetext{
${ }^{54}$ Breinlinger, E. C.; Rotello, V. M. J. Am. Chem. Soc. 1997, 119, 1165-1166.
} 


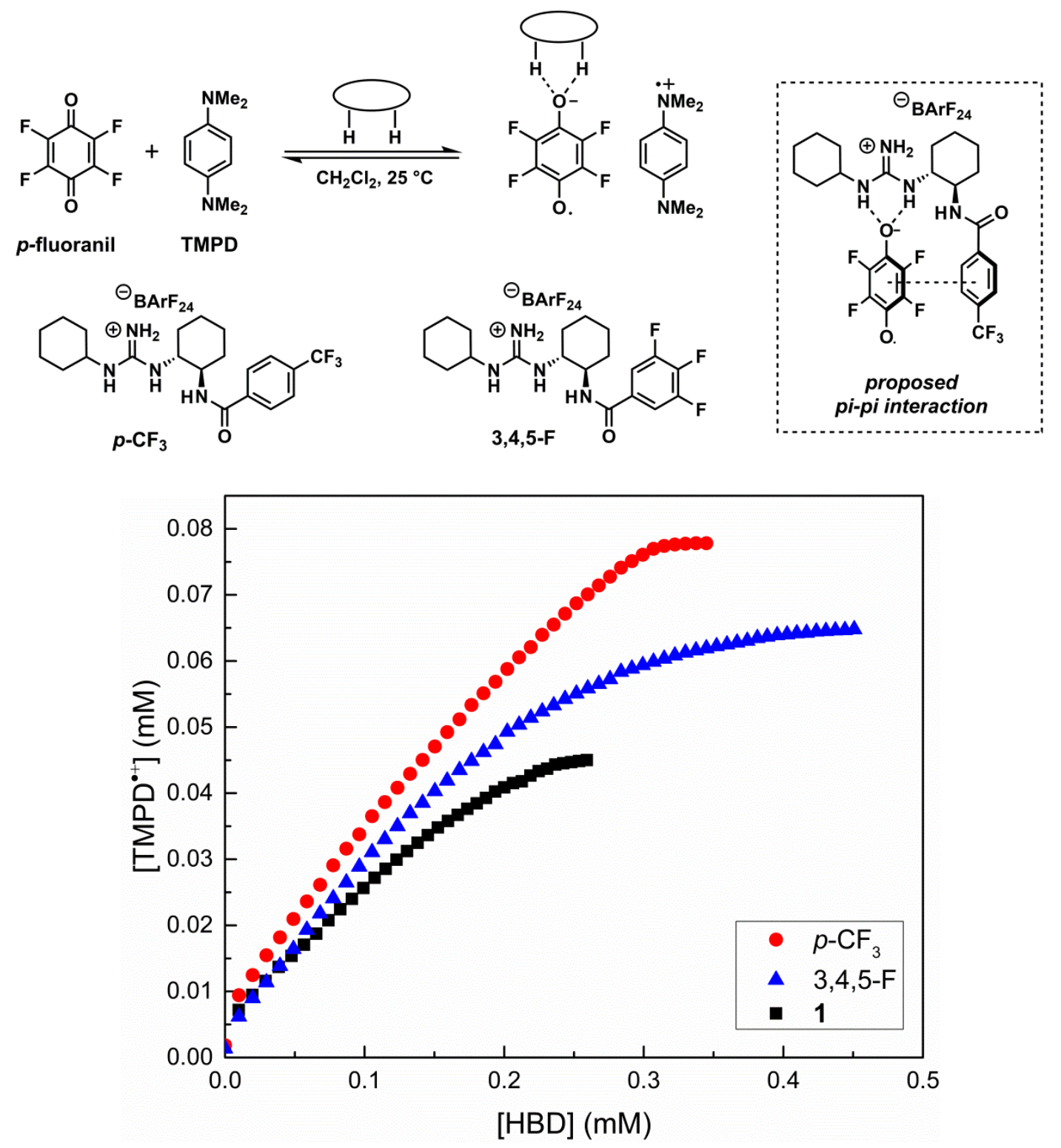

Figure 2.92. Titration of TMPD $(0.1 \mathrm{mM})$ and $p$-fluoranil $(0.1 \mathrm{mM})$ using 1 and two benzamidesubstituted guanidinium salts. Formation of $\mathrm{TMPD}^{\bullet+}$ (at $567 \mathrm{~nm}$ ) was monitored by UV-vis spectrophotometry.

\section{2d.1.2 Preliminary results for HBD-coupled electron transfer to electron-deficient $p$ - quinones}

An alternative approach to achieving enhanced substrate scope involves using HBDcoupled electron transfer to activate more reactive electron-deficient quinones, such as DDQ. ${ }^{37}$ The thermodynamic penalty $\left(\Delta \mathrm{G}_{\mathrm{ET}}\right.$, Figure 2.3$)$ for electron transfer to DDQ would be smaller. 
Substantial activation of DDQ would therefore allow quinone-mediated oxidation of a broader range of synthetically interesting substrates. The study of HBD-coupled electron transfer to electron-deficient $p$-quinones is also of fundamental mechanistic interest, due to their structural differences relative to $\mathbf{Q}$ and $o$-quinones generally. As such, their activation could require design of entirely new H-bonding scaffolds.

Preliminary investigation into $p$-quinones has already been undertaken, and reveals that they also can be activated through HBD-coupled electron transfer. Electrochemical experiments using $p$-chloranil ( $\boldsymbol{p}$-Q) have already been briefly discussed in section 2a.4.1 (Figure 2.6). Analysis of these data through simulations indicates that $\mathbf{1}$ binds to $\mathbf{p}-\mathbf{Q}^{\mathbf{*}}$ in a $2: 1$ complex through an ECC pathway (Figure 2.93). ${ }^{55}$ The parameters obtained from the simulations reveal that the binding constant describing formation of the $(\mathbf{1})_{2} \cdot \mathbf{p}-\mathbf{Q}^{\bullet-}$ complex is $2.1 \times 10^{8} \mathrm{M}^{-2}$ (Table 2.19).

${ }^{55}$ Fit performed by Dr. Andrew Ullman. 


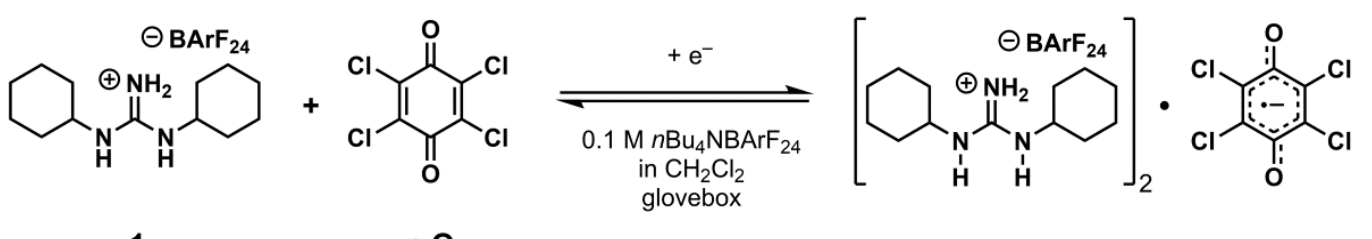

$1 \quad p-Q$

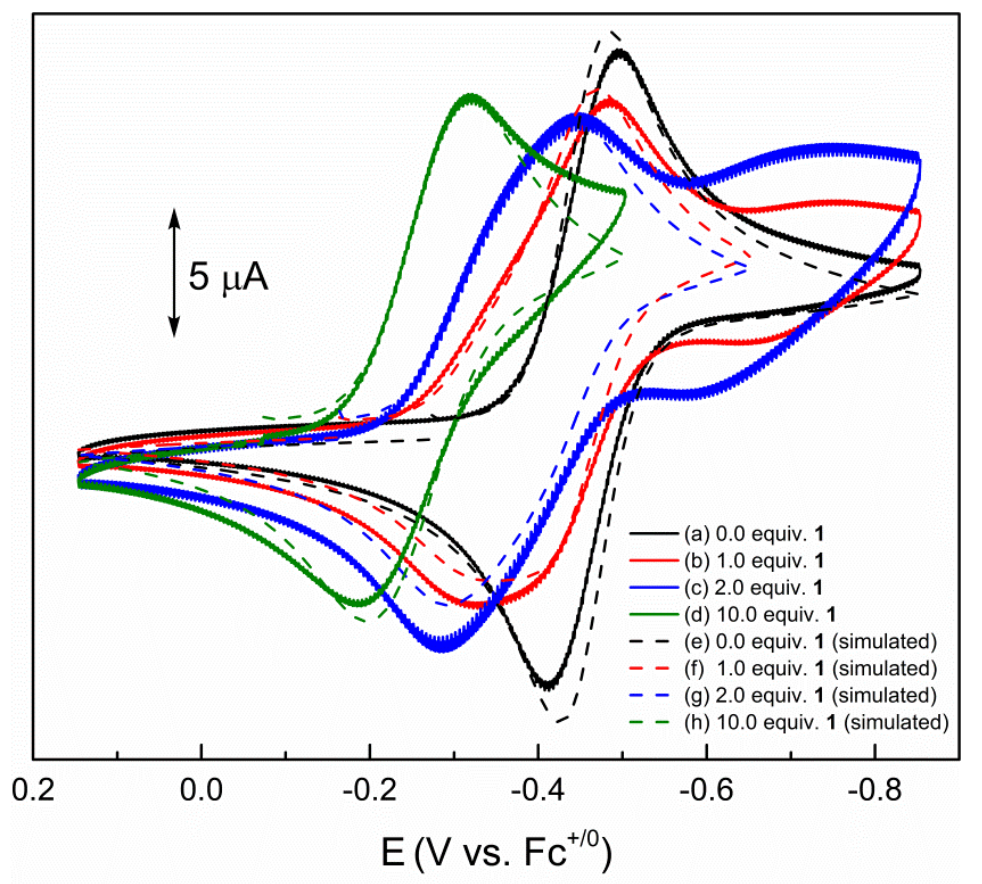

Figure 2.93. Experimental $\mathrm{CVs}$ of $p$-chloranil (initial concentration $=0.5 \mathrm{mM}$ ) recorded in 0.1 $\mathrm{M} n \mathrm{Bu}_{4} \mathrm{NBArF}_{24} / \mathrm{CH}_{2} \mathrm{Cl}_{2}$ (glovebox) in the presence of increasing [1] and comparison with simulation of an ECC mechanism.

Table 2.19. Equations and best-fit parameters used to simulate $\mathrm{CV}$ data for $0.5 \mathrm{mM}$-chloranil in $0.1 \mathrm{M} \mathrm{nBu} \mathrm{BuArF}_{24} / \mathrm{CH}_{2} \mathrm{Cl}_{2}$ (glovebox) in the presence of increasing [1]. Italicized values were fixed in the simulation.

\begin{tabular}{cccc}
\hline $\begin{array}{c}\text { Electron transfer } \\
\text { steps }\end{array}$ & $\mathbf{E}_{\mathbf{1} / \mathbf{2}}\left(\mathbf{V}\right.$ vs. $\left.\mathbf{F c}^{\mathbf{0}++}\right)$ & $\boldsymbol{\alpha}$ & $\mathbf{k}_{\mathbf{s}}(\mathbf{c m} / \mathbf{s})$ \\
\hline $\boldsymbol{p}-\mathbf{Q}+\mathrm{e}^{-} \rightarrow \boldsymbol{p}-\mathbf{Q}^{\bullet-}$ & -0.47 & 0.5 & 0.35 \\
\hline $\mathbf{C h e m i c a l ~ s t e p s}$ & $\mathbf{K}_{\mathrm{eq}}\left(\mathbf{M}^{-\mathbf{1}}\right)$ & $\mathbf{k}_{\mathbf{f}}\left(\mathbf{s}^{-\mathbf{1}}\right)$ \\
\hline $\mathbf{1}+\boldsymbol{p}-\mathbf{Q}^{\bullet-} \rightarrow \mathbf{1} \cdot \boldsymbol{p}-\mathbf{Q}^{\bullet-}$ & $2.7 \times 10^{4}$ & $1 \times 10^{12}$ \\
$\mathbf{1} \cdot \boldsymbol{p}-\mathbf{Q}^{\bullet-} \rightarrow(\mathbf{1})_{\mathbf{2}} \cdot \boldsymbol{p}-\mathbf{Q}^{\bullet-}$ & $7.9 \times 10^{3}$ & $1 \times 10^{11}$
\end{tabular}


This mechanistic difference compared with $\mathbf{Q}$ indicates that pre-association may only be favored with $o$-quinones, and is disfavored with $p$-quinones. The 1:1 complexes with the neutral quinone could have different geometries for the two quinone structures-with $\mathbf{Q}$, both oxygen atoms are accessible to the HBD, whereas in $\boldsymbol{p}$ - $\mathbf{Q}$, only one oxygen atom is capable of doing engaging in H-bonding (Figure 2.94).
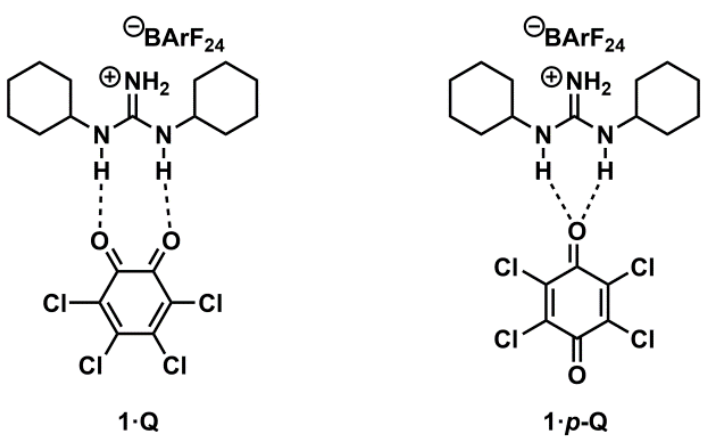

Figure 2.94. Differences in binding geometry between the $\mathbf{1} \cdot \mathbf{Q}$ complex and the $\mathbf{1} \cdot \mathbf{p}-\mathbf{Q}$ complex

Electrochemical experiments also reveal that DDQ can be activated toward electron transfer through $\mathrm{H}$-bonding, as the $\mathrm{CV}$ traces display a shift in $\mathrm{E}_{1 / 2}$ upon addition of 1 (Figure 2.95). As with $\boldsymbol{p}-\mathbf{Q}^{\bullet-}$, a 2:1 complex is also formed between $\mathbf{1}$ and $\mathrm{DDQ}^{\bullet-}$ as the result of an ECC mechanism. However, the binding of $\mathrm{DDQ}^{\bullet-}$ to 1 is significantly weaker, with $\mathrm{K}_{3} \mathrm{~K}_{4}$ determined to be $6.3 \times 10^{5} \mathrm{M}^{-2}$ (Table 2.20). This diminished binding relative to that observed with $\mathbf{Q}^{\bullet-}$ is attributed to the poorer Lewis basicity of $\mathrm{DDQ}^{\circ-}$ as a result of its electron deficiency.

Because use of DDQ intensifies the challenge presented by activation of electrondeficient quinones through $\mathrm{H}$-bonding, its activation will require design of new HBDs that can stabilize $\mathrm{DDQ}^{--}$. In this context, global changes to the H-bonding moiety (vide supra) will be 
essential. The potential for DDQ to expand the scope of HBD-coupled electron transfer warrants this future investigation.
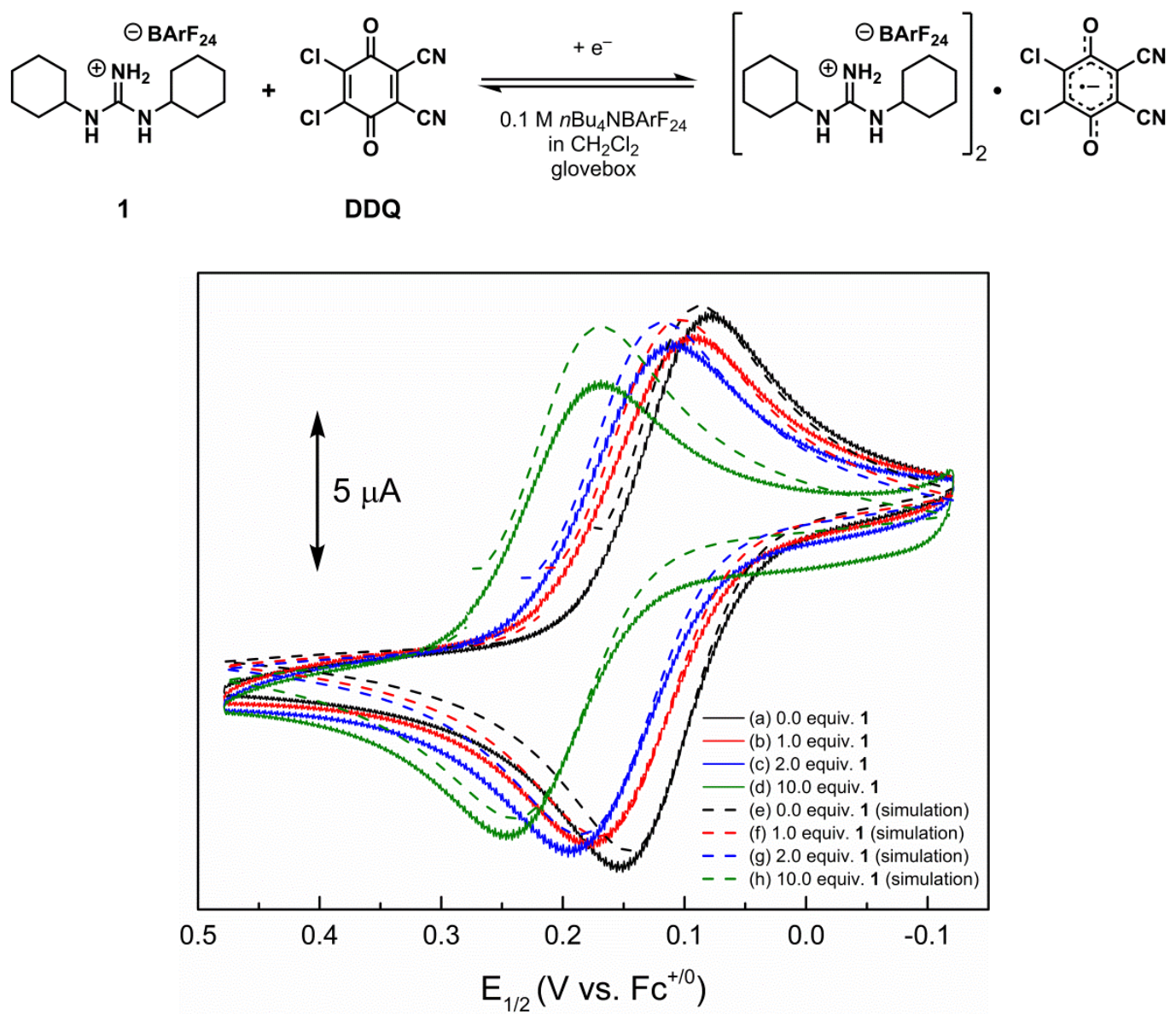

Figure 2.95. Experimental CVs of DDQ (initial concentration $=0.5 \mathrm{mM}$ ) recorded in $0.1 \mathrm{M}$ $n \mathrm{Bu}_{4} \mathrm{NBArF}_{24} / \mathrm{CH}_{2} \mathrm{Cl}_{2}$ (glovebox) in the presence of increasing [1] and comparison with simulation of an ECC mechanism.

Table 2.20. Equations and best-fit parameters used to simulate $\mathrm{CV}$ data for $0.5 \mathrm{mM}$ DDQ in 0.1 $\mathrm{M} n \mathrm{Bu}_{4} \mathrm{NBArF}_{24} / \mathrm{CH}_{2} \mathrm{Cl}_{2}$ (glovebox) in the presence of increasing [1]. Italicized values were fixed in the simulation.

\begin{tabular}{|c|c|c|c|}
\hline Electron transfer steps & $\mathrm{E}_{1 / 2}\left(\mathrm{~V} v \mathrm{vs} . \mathrm{Fc}^{0 /+}\right)$ & $\alpha$ & $\mathbf{k}_{\mathbf{s}}(\mathbf{c m} / \mathbf{s})$ \\
\hline $\mathbf{D D Q}+\mathrm{e}^{-} \rightarrow \mathbf{D D Q}^{--}$ & 0.12 & 0.5 & 0.35 \\
\hline Chemical steps & $K_{\mathrm{eq}}\left(\mathbf{M}^{-1}\right)$ & & $k_{f}\left(s^{-1}\right)$ \\
\hline $1+\mathrm{DDQ}^{\bullet-} \rightarrow 1 \cdot \mathrm{DDQ}^{\bullet-}$ & $3.5 \times 10^{3}$ & & $1 \times 10^{10}$ \\
\hline $1 \cdot \mathrm{DDQ}^{\bullet-} \rightarrow(1)_{2} \cdot \mathrm{DDQ}^{\bullet-}$ & 180 & & $1 \times 10^{10}$ \\
\hline
\end{tabular}




\section{2d.2 Outlook for synthetic application in enantioselective catalysis}

\section{2d.2.1 Conceptual design}

Ion-pairing of reactive intermediates in the presence of chiral catalysts offers a means of achieving enantioselectivity in organic transformations. ${ }^{56}$ Specifically, chiral HBDs have been employed to generate these catalyst-bound intermediates through two primary mechanisms (Figure 2.96). In the anion-binding mechanism (Figure 2.96A), a reactive ion pair is formed after generation of a cationic intermediate. ${ }^{57}$ The anionic component of the pair is intercepted by the HBD, and engages with the cationic intermediate electrostatically. The electrostatic attraction between a cationic intermediate and a H-bonded anion is also relevant in the anion-abstraction mechanism, in which the reactive ion pair is generated by HBD-mediated abstraction of a leaving group (Figure 2.96B). ${ }^{58}$

HBD-coupled electron transfer using quinone oxidants offers an approach similar to the anion-binding mechanism. Electron transfer from a substrate to $\mathbf{Q}$ would afford a catalyst-bound radical ion pair, in which the H-bonded $\mathbf{Q}^{\bullet-}$ associates with the ionized substrate through electrostatic attraction and other non-covalent interactions (Figure 2.96C). This application of HBD-coupled electron transfer toward reagent activation would complement the PCET strategies used to activate substrates toward electron transfer (section 1.5). For example, use of our strategy could enable oxidative transformations on acid-sensitive substrates, for which PCET would be impractical. Furthermore, HBD-coupled electron transfer applied to reagent activation could

\footnotetext{
${ }^{56}$ Brak, K.; Jacobsen, E. N. Angew. Chem. Int. Ed. 2013, 52, 534-561.

${ }^{57}$ For representative examples, see (a) Raheem, I. T.; Thiara, P. S.; Peterson, E. A.; Jacobsen, E. N. J. Am. Chem. Soc. 2007, 129, 13404-13405. (b) Xu, H.; Zuend, S. J.; Woll, M. G.; Tao, Y.; Jacobsen, E. N. Science 2010, 327, 986-990. (c) Knowles, R. R.; Lin, S.; Jacobsen, E. N. J. Am. Chem. Soc. 2010, 132, 5030-5032.

${ }^{58}$ For representative examples, see reference 50 and Reisman, S. E.; Doyle, A. G.; Jacobsen, E. N. J. Am. Chem. Soc. 2008, 130, 7198-7199.
} 
enable oxidation of functional groups (e.g. alkenes) in substrates that would not be readily activated through PCET.

(a) anion-binding

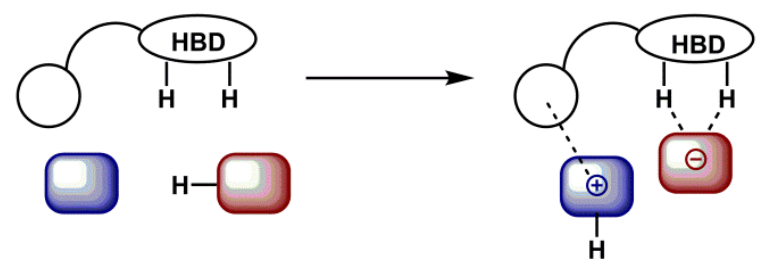

(b) anion-abstraction

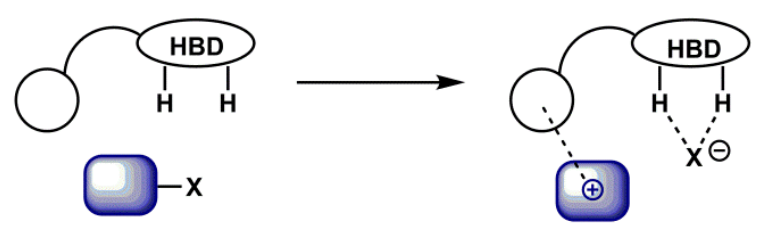

(c) HBD-coupled electron transfer

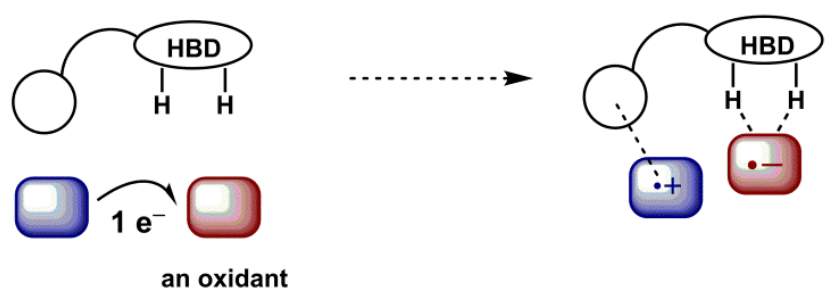

Figure 2.96. Mechanisms for ion-pairing catalysis.

HBD-coupled electron transfer using quinone oxidants offers an approach similar to the anion-binding mechanism. Electron transfer from a substrate to $\mathbf{Q}$ would afford a catalyst-bound radical ion pair, in which the $\mathrm{H}$-bonded $\mathbf{Q}^{\bullet-}$ associates with the ionized substrate through electrostatic attraction and other non-covalent interactions (Figure 2.96C). This application of HBD-coupled electron transfer toward reagent activation would complement the PCET strategies used to activate substrates toward electron transfer (section 1.5). For example, use of our strategy could enable oxidative transformations on acid-sensitive substrates, for which PCET would be impractical. Furthermore, HBD-coupled electron transfer applied to reagent activation could enable oxidation of functional groups (e.g. alkenes) in substrates that would not be readily activated through PCET. 
Mechanistically, the indication that H-bonding to $\mathbf{Q}$ occurs prior to electron transfer (section 2a.5) demonstrates potential for use of this system in enantioselective processes, as catalyst binding prior to generation of reactive intermediates is likely to be beneficial. Maintaining this association between the $\mathbf{Q}$, substrate, and HBD is crucial to achieving enantioselectivity. In the anion-binding and anion-abstraction mechanisms, the catalyst-bound anion provides a basis for association between the catalyst and substrate through electrostatic interactions. In these contexts, Coulombic repulsion between the cationic catalyst and cationic intermediate likely precludes intimate association between the two. ${ }^{59}$ However, in a quinonemediated reaction, the chemical involvement of the catalyst-bound quinone in all steps of the reaction necessitates close proximity between the substrate and the catalyst (Figure 2.91). In this case, as long as the quinone remains associated with the HBD, the substrate should be as well. This should hold true for all oxidative/dehydrogenative reactions using quinones, and in principle, enantioselectivity in these reactions is possible using chiral HBDs.

\section{2d.2.2 Oxidative lactonization catalyzed by chiral, dimeric bis-guanidinium salts}

Toward the goal of developing such an enantioselective reaction, we have prepared several dicationic, chiral bis-guanidinium salts to probe their efficacy in the oxidative lactonization. These catalysts afford the oxidative lactonization product in good yield (Figure 2.97) but no enantioselectivity, although only a limited variety of chiral appendages were investigated. Despite this, the promising reactivity obtained with these chiral HBDs indicates that they could offer a foundation for development of other chiral catalysts. In a fundamental sense, this demonstrates the broad application for our discovery that dicationic HBDs are most effective at promoting electron transfer.

\footnotetext{
${ }^{59}$ Andrew Bendelsmith and Andreas Rötheli, personal communication.
} 


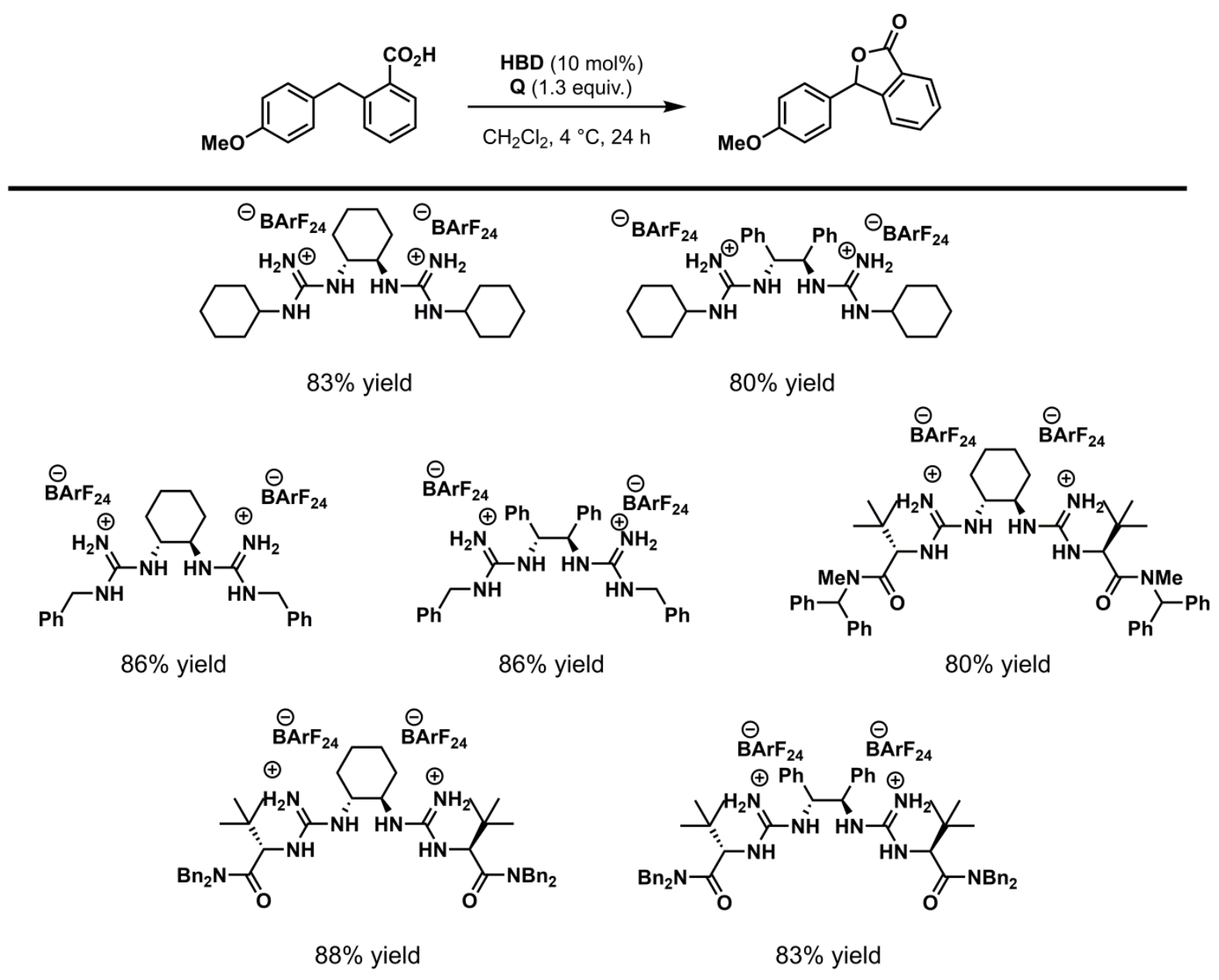

Figure 2.97. Dimeric guanidinium salts.

Design of a dicationic HBD which contains an amidinium group-which proved to be most effective in the studies discussed above - was also undertaken (Figure 2.98). These linked amidinium-guanidinium salts give improved yield in the oxidative lactonization reaction; still, no enantioselectivity is observed. We expect that these HBDs would exhibit enhanced binding to $\mathbf{Q}^{\mathbf{*}}$, based on the increased acidity of the amidinium relative to the guanidinium. This specific H-bonding scaffold, which is highly modular at the H-bonding sites with respect to electronic tuning and chiral functionality, may offer the most promise in terms of developing a highly active, selective catalyst for HBD-coupled electron transfer. 


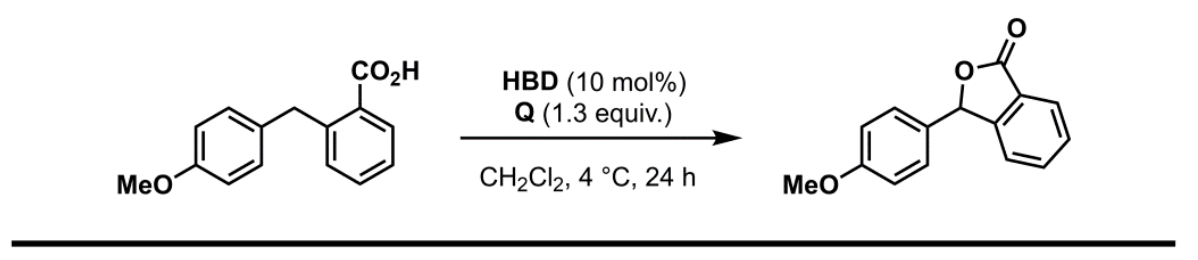

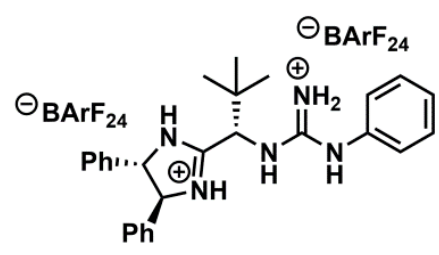

$94 \%$ yield

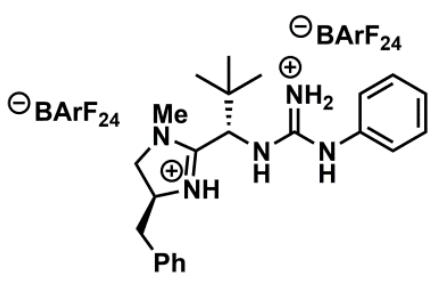

$98 \%$ yield

Figure 2.98. Linked amidinium-guanidinum salts.

This modularity has already been briefly investigated by replacing the guanidinium moiety with more acidic functional groups. Promising initial results have been obtained with linked uronium-amidinium salts, which show improved catalysis relative to bis-amidinium $\mathbf{1 0}$ (Figure 2.99), giving increased yield after $8 \mathrm{~h}$ reaction times.

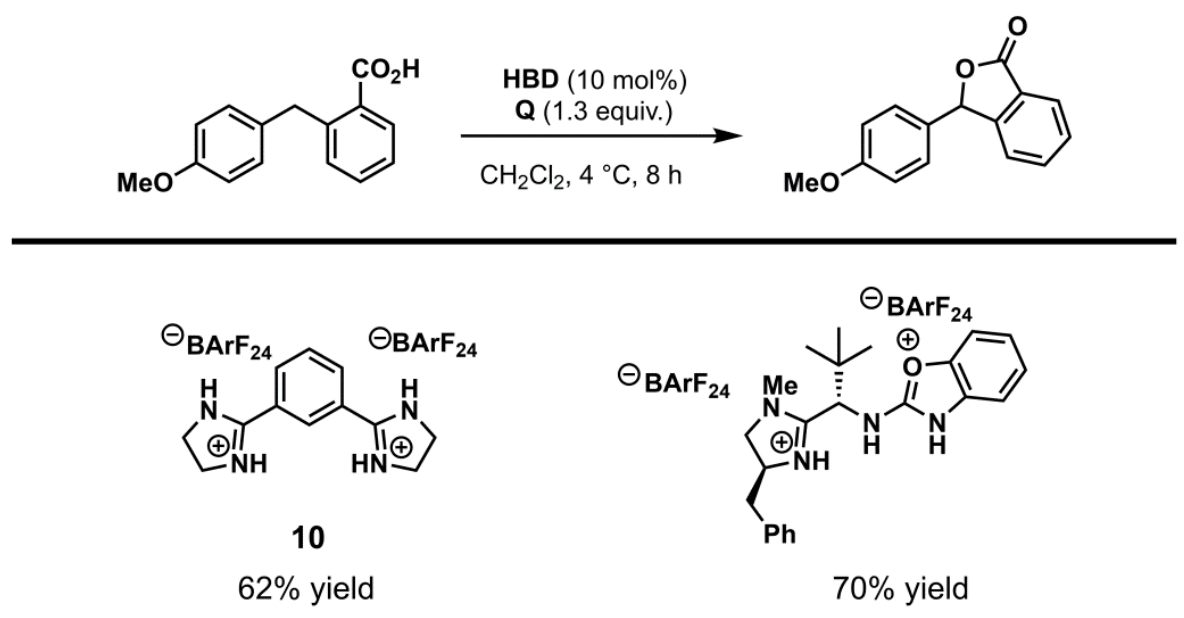

Figure 2.99. Comparison of yield after 8 hours obtained with $\mathbf{1 0}$ and linked amindiniumuronium salt. 


\section{2d.3 Proposed application of HBD-coupled electron transfer in a redox-neutral reaction}

\section{2d.3.1 Reactivity principle}

Redox-neutral pericyclic reactions promoted by electron-hole catalysis were initially reported by Bauld and coworkers, ${ }^{60}$ and have recently been examined in a photocatalytic context. ${ }^{61}$ HBD-coupled electron transfer to quinones could also find application in electron-hole catalysis by mediating oxidation of substrates and, after a series of bond-forming steps, returning an electron to the product (Figure 2.100). Such a reaction would constitute a fundamentally new reactivity manifold, as quinones are not known to behave as electron shuttles in synthetic contexts, although they do play this role in biology (section 1.3.2).

Activation of quinones through H-bonding may provide a unique opportunity to enable this reactivity, as the stabilization offered to $\mathbf{Q}^{\bullet-}$ by the HBD may ensure that the species is longlived enough to participate in back-electron transfer. Furthermore, this binding could preclude undesired reaction pathways that are mediated by the reduced species. The oxidized intermediate undergoes cyclization or is otherwise transformed to a new oxidized species. If this resultant radical cation is less stable than that generated from the initial electron transfer, it should readily undergo back-electron transfer to regenerate the quinone oxidant. This type of reactivity would be applicable to a number of transformations, including the Diels-Alder and [2+2] cycloadditions studied by Bauld and others.

${ }^{60}$ (a) Bellville, D. J.; Wirth, D. D.; Bauld, N. L. J. Am. Chem. Soc. 1981, 103, 718-720. (b) Bauld, N. L.; Pabon, R. A. J. Am. Chem. Soc. 1983, 105, 633-634. (c) Pabon, R. A.; Bellville, D. J.; Bauld, N. L. J. Am. Chem. Soc. 1983, 105, 5158-5159. (d) Bauld, N. L.; Bellville, D. J.; Harirchian, B.; Lorenz, K. T.; Pabon, Jr., R. A.; Reynolds, D. W.; Wirth, D. D.; Chiou, H.-S.; Marsh, B. K. Acc. Chem. Res. 1987, 20, 371-378. (e) Harirchian, B.; Bauld, N. L. Tetrahedron Lett. 1987, 28, 927-930.

${ }^{61}$ (a) Lin, S.; Ischay, M. A.; Fry, C. G.; Yoon, T. P. J. Am. Chem. Soc. 2011, 133, 19350-19353. (b) Lin, S.; Padilla, C. E.; Ischay, M. A.; Yoon, T. P. Tetrahedron Lett. 2012, 53, 3073-3076. 


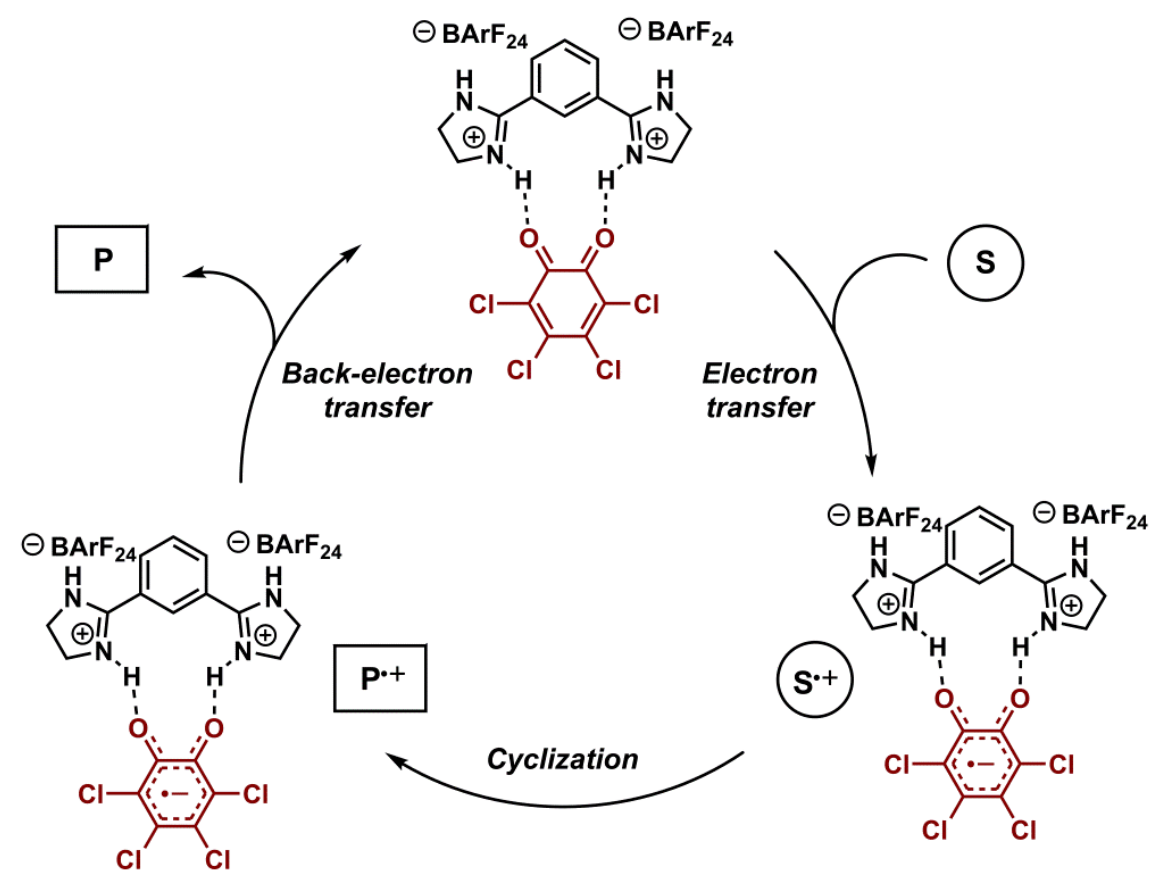

Figure 2.100. Proposed mechanism for a quinone-mediated redox-neutral reaction catalyzed through HBD-coupled electron transfer.

\section{2d.3.2 Proposal for enantioselective approach}

In such a redox-neutral reaction, association between the substrate and HBD would be dependent on the Coulombic attraction discussed in section 2d.2.1. In this case, the aforementioned electrostatic repulsion between a dicationic catalyst and cationic intermediate will be detrimental. Although a racemic reaction catalyzed by HBDs would constitute an important contribution in terms of discovery of novel reactivity, a different approach may be required in order to achieve selectivity. We have established that dicationic HBDs are required in order to achieve substantial activation of electron-deficient quinones; therefore, a complementary PCET approach-still applied to quinone activation-may be more effective to achieve enantioselective catalysis (Figure 2.101). Use of a chiral acid to activate $\mathbf{Q}$ would result in neutral $\mathbf{Q H}^{\bullet}$ through PCET. The chiral conjugate base can then associate with the oxidized substrate to influence the selectivity of the reaction. A second PCET reaction would result in 
back-electron transfer from $\mathbf{Q H}^{\bullet}$ concomitant with deprotonation of this species to regenerate the acid catalyst and $\mathbf{Q}$.

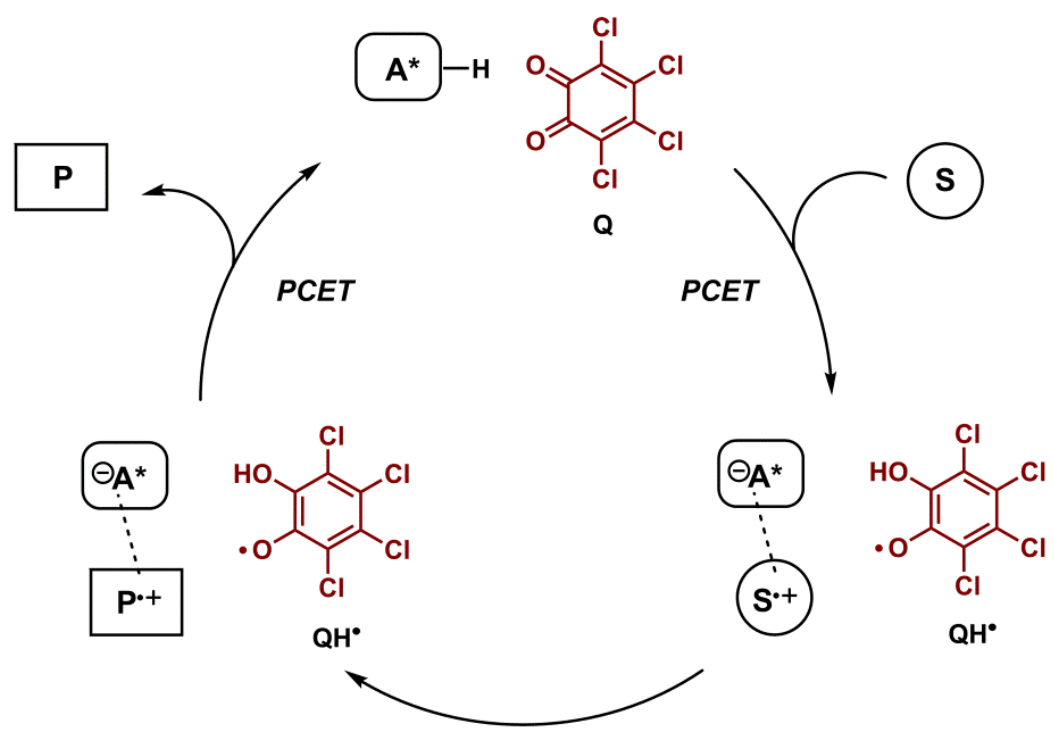

Figure 2.101. Proposal for quinone-mediated redox-neutral reaction using chiral acid catalysts.

\section{2d.4 Conclusions}

The results described herein illustrate that HBD-coupled electron transfer can be successfully applied to activate electron-deficient quinones. We have established that the results obtained from our mechanistic studies can find practical application in a synthetic context. Outlined here are possible avenues for further investigation, which could enable HBD-coupled electron transfer to be employed in an even broader context. Continued use of mechanistic tools, particularly with respect to electrochemical measurements, could enable the development of HBDs with an increased affinity for $\mathbf{Q}^{\bullet-}$, leading to a corresponding enhancement in catalytic activity. We have described some progress toward this goal, with the linked amidinium-uronium catalyst showing the most promise. These mechanistic tools could also provide insight into the activation of inherently more oxidizing quinones, which—in addition to being of fundamental 
mechanistic interest-would also offer an improved scope of attainable reactivity. We expect that these preliminary results, in combination with those presented throughout this thesis, will provide a basis for fruitful investigation into HBD-coupled electron transfer.

\section{2d.5 Experimental details}

\section{Cyclic voltammetry experiments with 1 and $p$-chloranil}

A $0.1 \mathrm{M}$ stock solution of $\mathbf{1}(217.5 \mathrm{mg})$ in $\mathrm{CH}_{2} \mathrm{Cl}_{2}$ was prepared in a $2 \mathrm{~mL}$ volumetric flask. A stock solution of $p$-chloranil (3.9 mg in $0.1 \mathrm{M} \mathrm{nBu} \mathrm{Bu}_{4} \mathrm{NBrF}_{24} / \mathrm{CH}_{2} \mathrm{Cl}_{2}$ ) was prepared in a $2 \mathrm{~mL}$ volumetric flask. A $0.5 \mathrm{mM}$ solution of $o$-chloranil in $0.1 \mathrm{M} n \mathrm{Bu}_{4} \mathrm{NBArF}_{24} / \mathrm{CH}_{2} \mathrm{Cl}_{2}$ was prepared in an electrochemical cell using $250 \mu \mathrm{L}$ of the $o$-chloranil stock solution and $3.75 \mathrm{~mL}$ of $0.1 \mathrm{M} n \mathrm{Bu}_{4} \mathrm{NBArF}_{24} / \mathrm{CH}_{2} \mathrm{Cl}_{2}$. The $\mathrm{CV}$ of the solution was recorded. The $p$-chloranil solution was titrated with 10-200 $\mu \mathrm{L}$ increments of $\mathbf{1}$, and a CV was recorded after each addition of $\mathbf{1}$. The experimental data were reproduced by simulation of an ECC mechanism. The full set of experimental data is shown in Figure 2.6 (section 2a.4.1) 


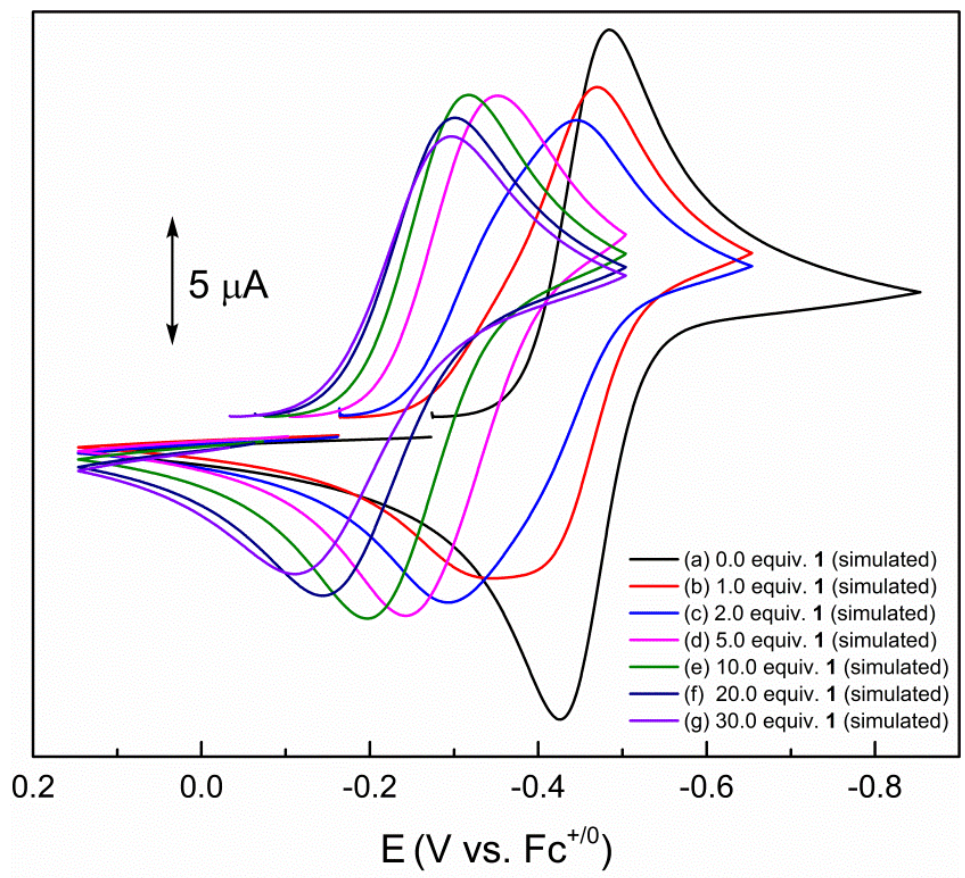

Figure 2.102. Full set of ECC simulations of $\mathrm{CV}$ data for $p$-chloranil (initial concentration $=0.5$ $\mathrm{mM}$ ) recorded in $0.1 \mathrm{MnBu} \mathrm{NBArF}_{24} / \mathrm{CH}_{2} \mathrm{Cl}_{2}$ (glovebox) in the presence of increasing [1].

\section{Cyclic voltammetry experiments with 1 and DDQ}

A $0.1 \mathrm{M}$ stock solution of $\mathbf{1}(217.5 \mathrm{mg})$ in $\mathrm{CH}_{2} \mathrm{Cl}_{2}$ was prepared in a $2 \mathrm{~mL}$ volumetric flask. A stock solution of DDQ (3.6 mg in $0.1 \mathrm{M} \mathrm{nBu} \mathrm{NBArF}_{24} / \mathrm{CH}_{2} \mathrm{Cl}_{2}$ ) was prepared in a $2 \mathrm{~mL}$ volumetric flask. A $0.5 \mathrm{mM}$ solution of DDQ in $0.1 \mathrm{M} n \mathrm{Bu}_{4} \mathrm{NBArF}_{24} / \mathrm{CH}_{2} \mathrm{Cl}_{2}$ was prepared in an electrochemical cell using $245 \mu \mathrm{L}$ of the DDQ stock solution and $3.75 \mathrm{~mL}$ of $0.1 \mathrm{M}$ $n \mathrm{Bu}_{4} \mathrm{NBArF}_{24} / \mathrm{CH}_{2} \mathrm{Cl}_{2}$. The $\mathrm{CV}$ of the solution was recorded. The DDQ solution was titrated with 10-200 $\mu \mathrm{L}$ increments of $\mathbf{1}$, and a $\mathrm{CV}$ was recorded after each addition of $\mathbf{1}$. The experimental data were reproduced by simulation of an ECC mechanism. 


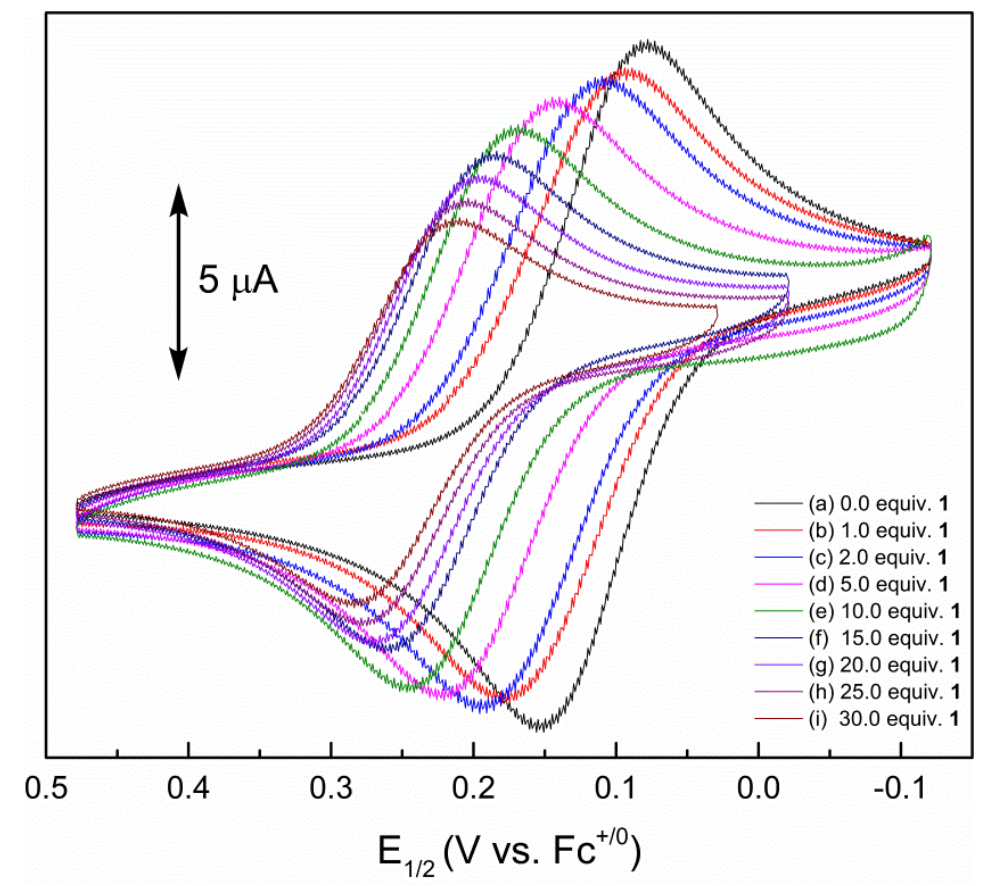

Figure 2.103. Full set of experimental CVs of DDQ (initial concentration $=0.5 \mathrm{mM}$ ) recorded in $0.1 \mathrm{M} n \mathrm{Bu}_{4} \mathrm{NBArF}_{24} / \mathrm{CH}_{2} \mathrm{Cl}_{2}$ (glovebox) in the presence of increasing [1].

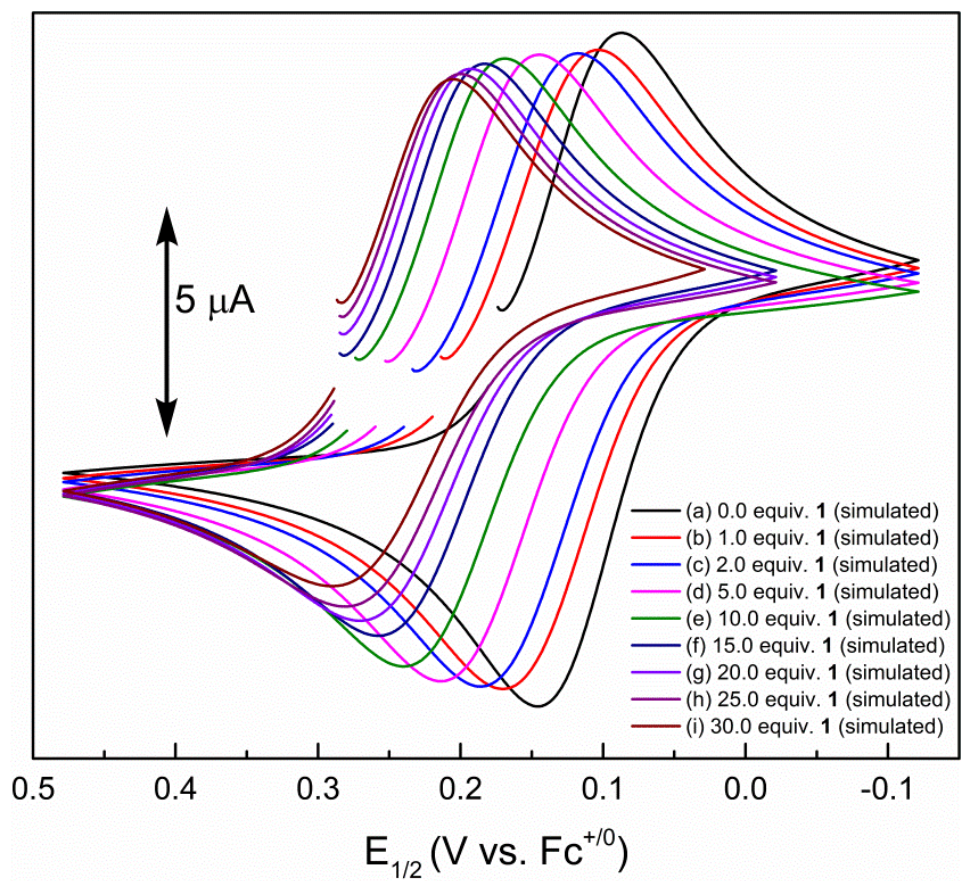

Figure 2.104. Full set of ECC simulations of CV data for DDQ (initial concentration $=0.5 \mathrm{mM}$ ) recorded in $0.1 \mathrm{M} n \mathrm{Bu}_{4} \mathrm{NBArF}_{24} / \mathrm{CH}_{2} \mathrm{Cl}_{2}$ (glovebox) in the presence of increasing [1]. 